University of Tennessee Health Science Center

UTHSC Digital Commons

$12-2010$

\title{
Development and Evaluation of Paclitaxel-Loaded Liposomal Formulations for Targeted Drug Delivery to Breast Cancer
}

Vinayagam Kannan

University of Tennessee Health Science Center

Follow this and additional works at: https://dc.uthsc.edu/dissertations

Part of the Pharmaceutics and Drug Design Commons

\section{Recommended Citation}

Kannan, Vinayagam , "Development and Evaluation of Paclitaxel-Loaded Liposomal Formulations for Targeted Drug Delivery to Breast Cancer" (2010). Theses and Dissertations (ETD). Paper 135. http://dx.doi.org/10.21007/etd.cghs.2010.0158.

This Dissertation is brought to you for free and open access by the College of Graduate Health Sciences at UTHSC Digital Commons. It has been accepted for inclusion in Theses and Dissertations (ETD) by an authorized administrator of UTHSC Digital Commons. For more information, please contact jwelch30@uthsc.edu. 


\title{
Development and Evaluation of Paclitaxel-Loaded Liposomal Formulations for Targeted Drug Delivery to Breast Cancer
}

\author{
Abstract \\ The objective of this work was to develop and evaluate paclitaxel-loaded liposomal formulations for \\ targeted drug delivery to breast cancer. The liposomal formulation was optimized to maximize drug \\ loading and physical stability. Cholesterol and saturated lipid content showed a negative influence on \\ paclitaxel loading. Short-term stability studies showed that optimum drug-lipid ratio is necessary for \\ adequate physical stability. Biodistribution studies in mouse xenografts bearing MDA-MB-231 breast \\ cancer using near infrared fluorescence imaging showed that the accumulation of tumor vasculature \\ targeted long-circulating liposomes (LCL) in the tumor was significantly less than non-targeted LCL at 48 \\ $\mathrm{h}$. The accumulation of these liposomes in the peritoneal cavity was higher suggesting that they were \\ cleared rapidly by the reticuloendothelial system. The antitumor efficacy of paclitaxel-loaded tumor \\ vasculature targeted LCL was compared with paclitaxel-loaded tumor cell targeted LCL. The antitumor \\ efficacy was comparable for tumor vasculature targeted LCL, non-targeted LCL, and paclitaxel solution \\ formulation with tumor volumes of $\sim 60-70 \%$ of the control treatment on 39 days post tumor inoculation. \\ In contrast, tumor cell targeted LCL showed a significantly higher antitumor efficacy compared to all other \\ treatments with tumor volumes of $\sim 30 \%$ of the control treatment. To improve the long-term stability, \\ these liposomes were lyophilized. The leakage and vesicle size increase during lyophilization was \\ minimized by using lyoprotectant sucrose in the formulation. Feasibility of developing gas-filled \\ liposomes for ultrasound mediated drug delivery was evaluated using freeze drying gas entrapment \\ method for the preparation. The in vitro measurements of echogenicity showed that these paclitaxel- \\ loaded tumor vasculature targeted gas-filled liposomes were acoustically active and can be disintegrated \\ by high intensity ultrasound pulses.

\section{Document Type \\ Dissertation} \\ Degree Name \\ Doctor of Philosophy (PhD) \\ Program \\ Pharmaceutical Sciences \\ Research Advisor \\ George C. Wood, Ph.D. \\ Keywords \\ Breast cancer, liposomes, paclitaxel, targeting, ultrasound

\section{Subject Categories} \\ Medicine and Health Sciences | Pharmaceutics and Drug Design | Pharmacy and Pharmaceutical \\ Sciences

\section{Comments} \\ One year patent embargo expired December 2011
}




\title{
DEVELOPMENT AND EVALUATION OF PACLITAXEL-LOADED LIPOSOMAL FORMULATIONS FOR TARGETED DRUG DELIVERY TO BREAST CANCER
}

\author{
A Dissertation \\ Presented for \\ The Graduate Studies Council \\ The University of Tennessee \\ Health Science Center
}

\author{
In Partial Fulfillment \\ Of the Requirements for the Degree \\ Doctor of Philosophy \\ From The University of Tennessee
}

By

Vinayagam Kannan

December 2010 
Copyright (C) 2010 by Vinayagam Kannan All rights reserved 


\section{ACKNOWLEDGEMENTS}

It is an immense pleasure to thank all the people who helped me in this dissertation work. I express my sincere gratitude to my advisor Dr. George C. Wood for his professional guidance, generous encouragement, and unconditional support all through this work. I thank Dr. Laura A. Thoma for providing me an opportunity to join the Parenteral Medications Laboratories (PML) and for her unwavering commitment to support this research work. I am grateful to my faculty committee members Dr. Hassan Almoazen, Dr. Mostafa Waleed Gaber, Dr. James R. Johnson, Dr. Ram I. Mahato, and Dr. Thaddeus A. Wilson for their advice, support, and help at various stages of this work.

I extend my sincere gratitude to other members of the PML group (past and present). Especially, I am thankful to Dr. Murali K. Divi, Dr. Hari R. Desu for their help, support, and suggestions in this work. I thank Dr. Robert J. Nolly, Dr. Himanshu Bhattacharjee, Dr. Vivian Loveless, Barry Braganza, Frank P. Horton, Pavan Balabathula, Gwen Stornes, Anthony C. Samsa, Elizabeth D. Barker, and Jennifer L. Hart for their support at various stages. I enjoyed scientific and technical discussions with all the members of the PML group. I am also grateful to the members of Dr. Mostafa Waleed Gaber group, Dr. Ram I. Mahato group, Dr. James R. Johnson group, and Dr. Tiffany N. Seagroves group and the faculty and staff of the Department of Comparative Medicine for their help and support.

I am thankful to The University of Tennessee Health Science Center and the PML for the facilities, equipment, and financial support. I thank all the faculty and staff of the Department of Pharmaceutical Sciences for their support. I am also thankful to the Parenteral Drug Association (PDA) for providing pre-doctoral fellowship. 


\begin{abstract}
The objective of this work was to develop and evaluate paclitaxel-loaded liposomal formulations for targeted drug delivery to breast cancer. The liposomal formulation was optimized to maximize drug loading and physical stability. Cholesterol and saturated lipid content showed a negative influence on paclitaxel loading. Short-term stability studies showed that optimum drug-lipid ratio is necessary for adequate physical stability. Biodistribution studies in mouse xenografts bearing MDA-MB-231 breast cancer using near infrared fluorescence imaging showed that the accumulation of tumor vasculature targeted long-circulating liposomes (LCL) in the tumor was significantly less than nontargeted LCL at $48 \mathrm{~h}$. The accumulation of these liposomes in the peritoneal cavity was higher suggesting that they were cleared rapidly by the reticuloendothelial system. The antitumor efficacy of paclitaxel-loaded tumor vasculature targeted LCL was compared with paclitaxel-loaded tumor cell targeted LCL. The antitumor efficacy was comparable for tumor vasculature targeted LCL, non-targeted LCL, and paclitaxel solution formulation with tumor volumes of $\sim 60-70 \%$ of the control treatment on 39 days post tumor inoculation. In contrast, tumor cell targeted LCL showed a significantly higher antitumor efficacy compared to all other treatments with tumor volumes of $\sim 30 \%$ of the control treatment. To improve the long-term stability, these liposomes were lyophilized. The leakage and vesicle size increase during lyophilization was minimized by using lyoprotectant sucrose in the formulation. Feasibility of developing gas-filled liposomes for ultrasound mediated drug delivery was evaluated using freeze drying gas entrapment method for the preparation. The in vitro measurements of echogenicity showed that these paclitaxel-loaded tumor vasculature targeted gas-filled liposomes were acoustically active and can be disintegrated by high intensity ultrasound pulses.
\end{abstract}




\section{TABLE OF CONTENTS}

CHAPTER 1. INTRODUCTION 1

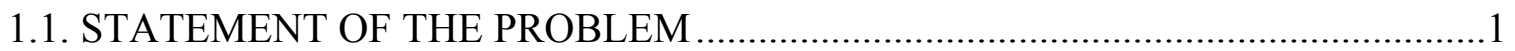

1.1.1. Breast Cancer and Treatment Choices ......................................................

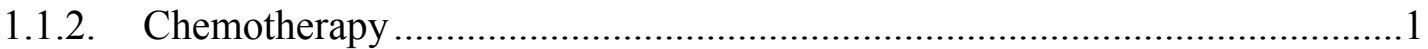

1.1.3. Paclitaxel: Mechanisms of Action .............................................................. 2

1.1.4. Drawbacks of Paclitaxel Therapy ...............................................................2

1.1.5. Barriers in Drug Delivery to Solid Tumors ................................................2

1.1.6. Strategies for Effective Delivery of Paclitaxel to Solid Tumors ..................... 3

1.1.7. Liposomes for Paclitaxel Delivery to Solid Tumors......................................... 3

1.1.8. Stability of Liposomes ...........................................................................

1.1.9. Echogenic Liposomes ..........................................................................

1.2. REVIEW OF LITERATURE: FORMULATION DEVELOPMENT, AND

DELIVERY ASPECTS OF LIPOSOMES IN CANCER CHEMOTHERAPY ..........5

1.2.1. Composition of Liposomes ...................................................................6

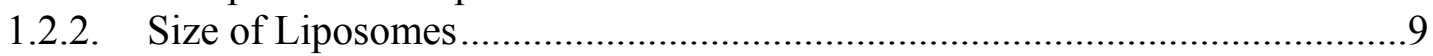

1.2.3. Surface Modification of Liposomes.......................................................... 10

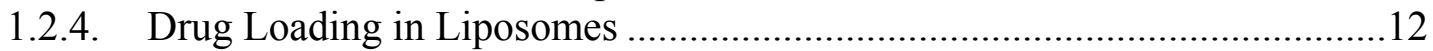

1.2.5. Targeted Drug Delivery with Liposomes ............................................... 15

1.2.6. Controlling Drug Release/Uptake from Liposomes ..................................25

1.2.7. Lyophilization to Improve the Stability of Liposomes ...............................29

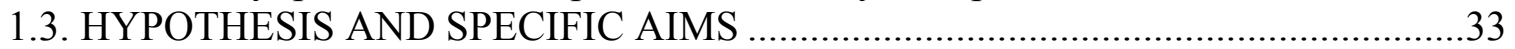

CHAPTER 2. DEVELOPMENT OF PROTOTYPE FORMULATION OF GAS-

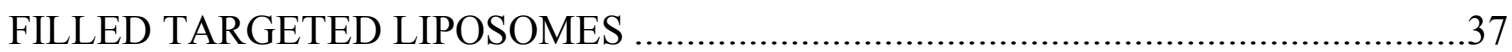

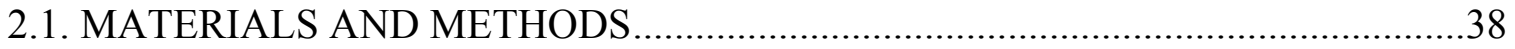

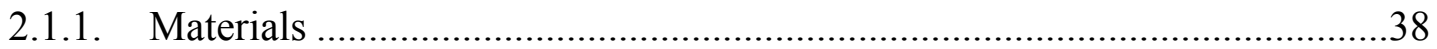

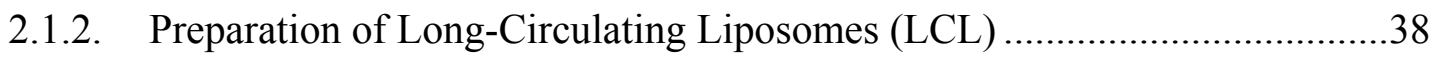

2.1.3. Verification of Conjugation Reaction between cRGD and Maleimide

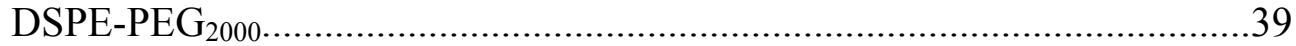

2.1.4. Preparation of cRGD Conjugated Targeted Long-Circulating Liposomes (RGD-TLCL) .......................................................................39

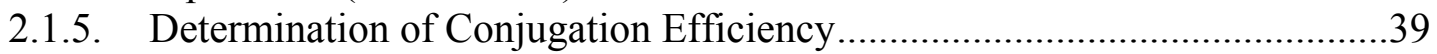

2.1.6. Determination of Particle Size and Zeta Potential ........................................40

2.1.7. Preparation of Gas-Filled LCL (GFLCL) and Gas-Filled RGD-TLCL

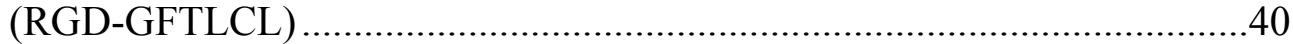

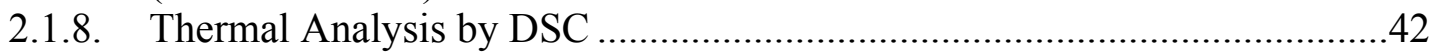

2.1.9. Determination of Moisture Content ..........................................................42

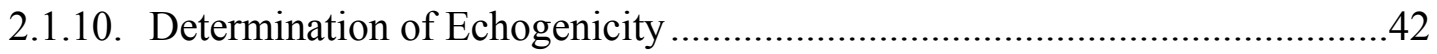

2.1.11. Characterization of Morphology of Liposomes ........................................42 
2.2. RESULTS AND DISCUSSION …………………..........................................4

2.2.1. Effect of Liposomal Bilayer Composition....................................................4

2.2.2. Effect of Lyoprotectant and Surfactant......................................................4

2.2.3. Effect of Sucrose Concentration on Echogenicity .........................................46

2.2.4. Determination of Conjugation Efficiency.....................................................51

2.2.5. Characteristics of GFLCL and RGD-GFTLCL .........................................51

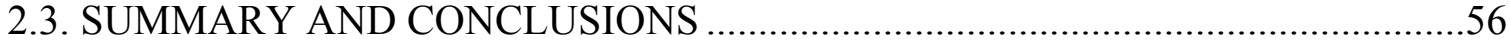

CHAPTER 3. OPTIMIZATION OF PACLITAXEL LOADING AND PHYSICAL STABILITY IN THE DEVELOPMENT OF LONG-

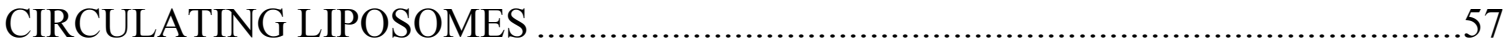

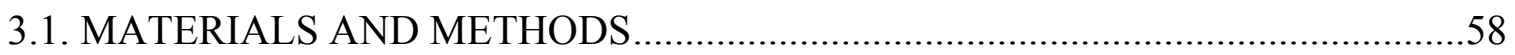

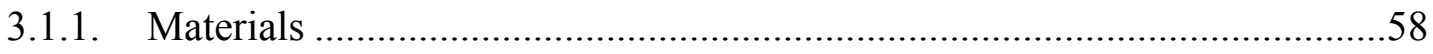

3.1.2. Preparation of Paclitaxel-Loaded Long-Circulating Liposomes (Pac-

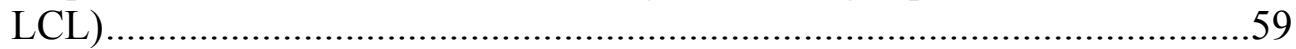

3.1.3. Determination of Paclitaxel Loading Efficiency .........................................59

3.1.4. Quantification of Paclitaxel from Liposomes ...............................................59

3.1.5. Determination of Particle Size and Zeta Potential .........................................60

3.1.6. Stability Evaluation of Pac-LCL...……………...........................................60

3.1.7. Characterization of Morphology of Pac-LCL .............................................60

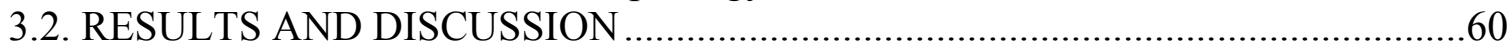

3.2.1. Optimization of Paclitaxel Loading …………...........................................60

3.2.2. Optimization of Physical Stability ………………....................................65

3.2.3. Optimization of the Extrusion Process ........................................................69

3.2.4. Stability Evaluation of Pac-LCL Dispersion .................................................69

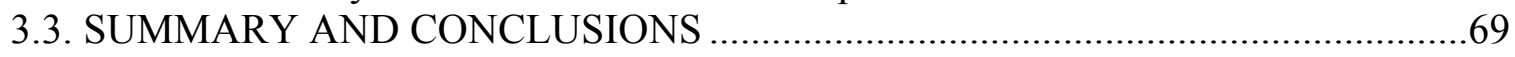

\section{CHAPTER 4. DEVELOPMENT OF PACLITAXEL-LOADED RGD}

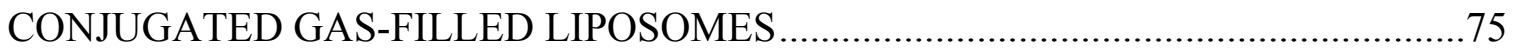

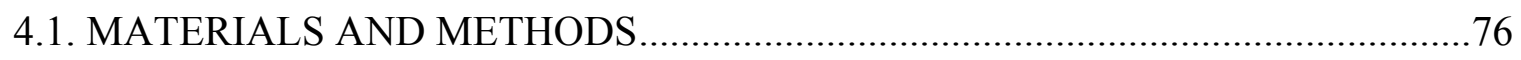

4.1.1. Materials ..........................................................................................

4.1.2. Preparation of Paclitaxel-Loaded Long-Circulating Liposomes (Pac-

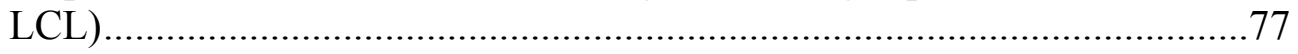

4.1.3. Preparation of Paclitaxel-Loaded cRGD Conjugated Targeted LongCirculating Liposomes (Pac-RGD-TLCL) .....................................................77

4.1.4. Determination of Conjugation Efficiency .....................................................77

4.1.5. Preparation of Gas-Filled Pac-LCL (Pac-GFLCL) and Gas-Filled PacRGD-TLCL (Pac-RGD-GFTLCL) .......................................................78

4.1.6. Thermal Analysis by DSC ……………………....................................78

4.1.7. Characterization of Pac-LCL, Pac-RGD-TLCL, Pac-GFLCL, Pac-

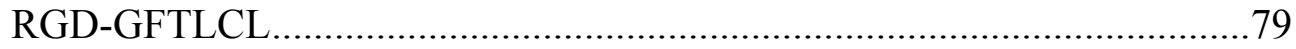

4.1.8. Determination of Echogenicity ................................................................ 


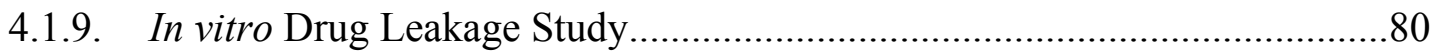

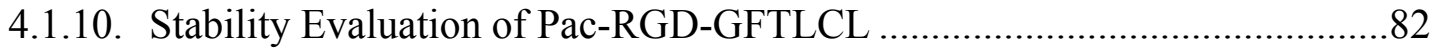

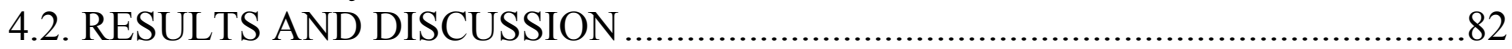

4.2.1. Effect of Lyoprotectant and the Manufacturing Process on PSD of Pac-

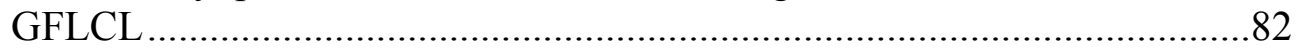

4.2.2. Effect of Mannitol Concentration ...............................................................89

4.2.3. In vitro Leakage Study with Pac-GFTLCL ……….....................................93

4.2.4. Conjugation Efficiency of cRGD to Maleimide-Pac-LCL ………..............93

4.2.5. Characteristics of Pac-RGD-GFTLCL ………….......................................96

4.2.6. Visualization and Disintegration of Pac-RGD-GFTLCL with

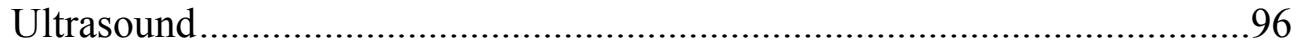

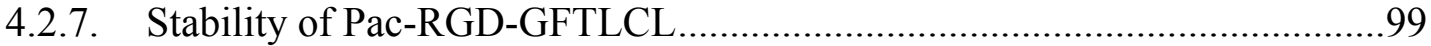

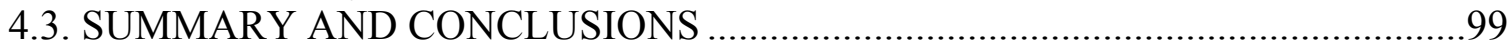

CHAPTER 5. IN VIVO EVALUATION OF BIODISTRIBUTION AND

EFFICACY OF TUMOR VASCULATURE TARGETED LIPOSOMES AND

COMPARISON OF EFFICACY WITH TUMOR CELL TARGETED

LIPOSMES

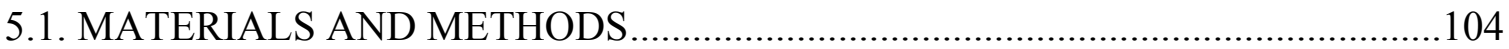

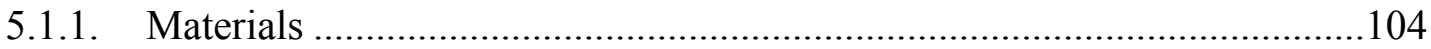

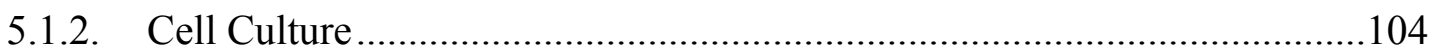

5.1.3. Preparation of Long-Circulating Liposomes Loaded with DiR (DiR

LCL) or Paclitaxel (Pac-LCL) ................................................................105

5.1.4. Preparation of cRGD Conjugated Targeted Long-Circulating

Liposomes Loaded with DiR (DiR-RGD-TLCL) or Paclitaxel (Pac-

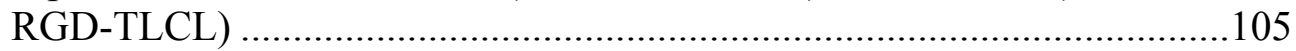

5.1.5. Preparation of Paclitaxel-Loaded Transferrin Conjugated Targeted

Long-Circulating Liposomes (Pac-Tf-TLCL) ………………………..........105

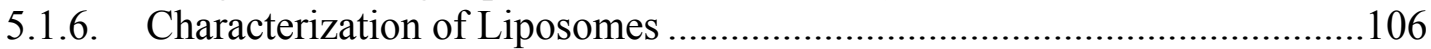

5.1.7. Stability Evaluation of Pac-RGD-TLCL and Pac-Tf-TLCL …..................107

5.1.8. Breast Cancer Mouse Xenograft Model ...................................................107

5.1.9. Evaluation of Biodistribution of Tumor Vasculature Targeted Liposomes by Near Infrared Fluorescence (NIRF) Imaging .......................107

5.1.10. Evaluation of Biodistribution of Paclitaxel from Tumor Vasculature

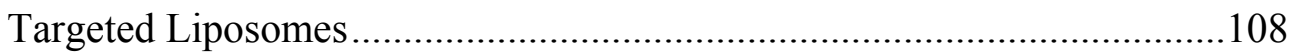

5.1.11. Evaluation of Antitumor Efficacy of Paclitaxel-Loaded Tumor Vasculature Targeted Liposomes and Tumor Cell Targeted Liposomes....109

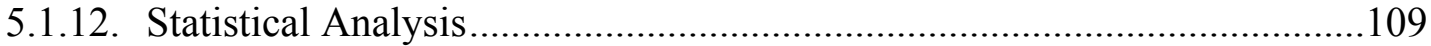

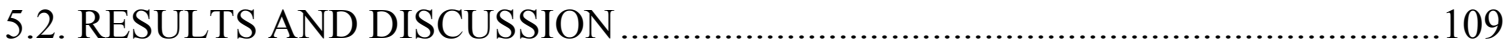

5.2.1. Characterization of the Liposomal Formulations ........................................109

5.2.2. Stability of Paclitaxel-Loaded Targeted Liposomes ......................................111

5.2.3. Near Infrared Fluorescence Imaging ............................................................111

5.2.4. Quantification of Paclitaxel from Plasma and Tumor Tissues to Determine Biodistribution of Paclitaxel-Loaded Liposomes 
5.2.5. Antitumor Efficacy of Tumor Vasculature and Tumor Cell Targeted

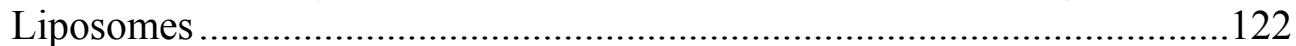

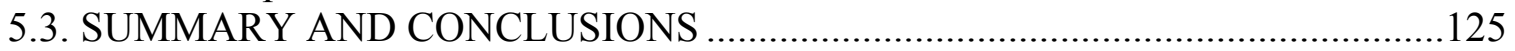

CHAPTER 6. LYOPHILIZATION OF PACLITAXEL-LOADED LIPOSOMES TO IMPROVE LONG TERM STABILITY …………………....................................127

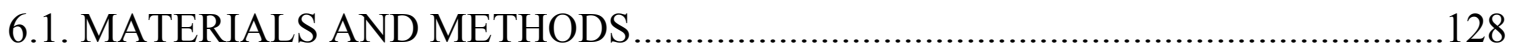

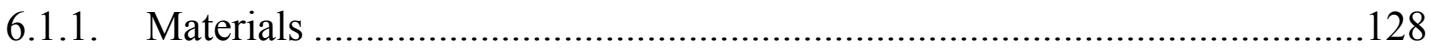

6.1.2. Preparation of Paclitaxel-Loaded Long-Circulating Liposomes (Pac-

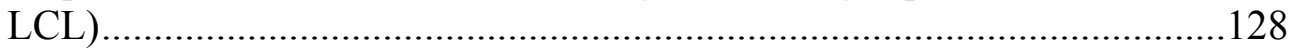

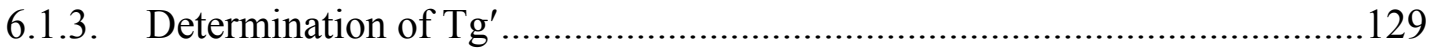

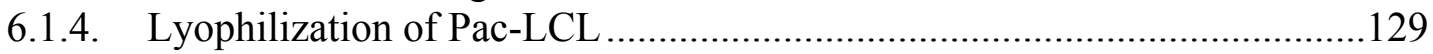

6.1.5. Characterization of Liposomal Formulations ............................................129

6.1.6. Preparation and Lyophilization of Paclitaxel-Loaded cRGD Conjugated Targeted Long-Circulating Liposomes (Pac-RGD-TLCL) .....130

6.1.7. Preparation and Lyophilization of Paclitaxel-Loaded Transferrin Conjugated Targeted Long-Circulating Liposomes (Pac-Tf-TLCL)...........131

6.1.8. Stability Evaluation of Lyophilized Formulations of Pac-LCL, Pac-

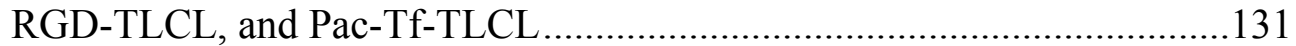

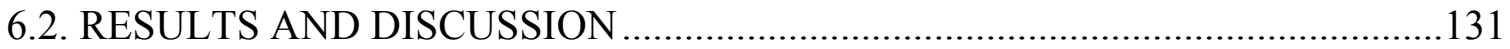

6.2.1. Effect of Sucrose on Paclitaxel Retention and PSD .................................131

6.2.2. Development of Lyophilized Targeted Liposomes.......................................137

6.2.3. Stability of Lyophilized Pac-LCL, Pac-RGD-TLCL, and Pac-Tf-TLCL...140

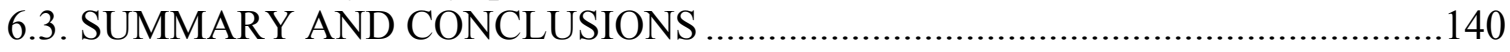

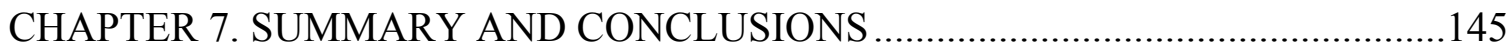

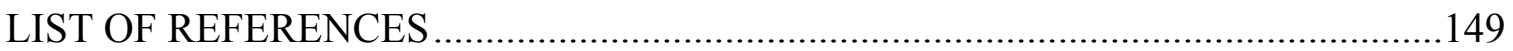

VITA 167 


\section{LIST OF FIGURES}

Figure 2-1. Cartoon representing the method of preparation of RGD-

GFTLCL

Figure 2-2. Schematic representation of the in vitro technique used for the measurement of echogenicity

Figure 2-3.

Figure 2-4.

Figure 2-5.

Figure 2-6. Figure 2-7.

Figure 2-8.

Figure 2-9.

Figure 2-10.

Figure 2-11. Figure 3-1. Figure 3-2.

Figure 3-3.

Figure 3-4.

Figure 3-5.

Figure 3-6.

Figure 3-7.

Figure 3-8.

Figure 3-9.

Figure 3-10.

Figure 4-1.

Figure 4-2.

Figure 4-3.

Figure 4-4.

Figure 4-5.

Figure 4-6.
Effect of liposome composition on PSD of liposomes after freeze drying .... .45

DSC thermograms showing the effect of sucrose and poloxamer on crystallization of mannitol

Effect of mannitol-sucrose ratio on PSD of liposomes after lyophilization 48

Effect of poloxamer on PSD of liposomes after lyophilization............49

Effect of mannitol-sucrose ratio on echogenicity of GFLCL …….......50

Conjugation reaction between maleimide-DSPE- $\mathrm{PEG}_{2000}$ and cRGD

HPLC chromatogram of the dialyzate obtained from equilibrium dialysis in comparison to standard solution of cRGD. ........................53

PSD of GFLCL and RGD-GFTLCL before and after lyophilization 54 TEM images of GFLCL (A) and RGD-GFTLCL (B) ……………......55

Effect of total phospholipids on paclitaxel loading ..............................62 Effect of cholesterol concentration on paclitaxel loading....................63 Effect of saturated-unsaturated lipid ratio on paclitaxel loading ..........64 Effect of drug-lipid ratio on paclitaxel loading....................................66 Effect of drug-lipid ratio on physical stability of Pac-LCL ..................67 Effect of drug-lipid ratio on physical stability of improved Pac-

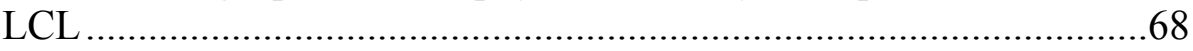
Effect of number of extrusion cycles on PSD and paclitaxel

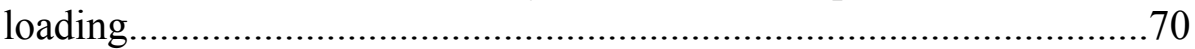

Effect of extrusion pressure on PSD and paclitaxel loading.................71

TEM image depicting morphology of Pac-LCL …………………….....72

Stability of Pac-LCL dispersion at $5 \pm 3^{\circ} \mathrm{C}$.........................................73

The in vitro model used for demonstrating disintegration of gasfilled liposomes

DSC thermograms showing the effect of lipid-sucrose ratio on

$\operatorname{Tg}^{\prime}$ . .83

Effect of lipid-sugar ratio on PSD after lyophilization of PacLCL

Effect of inravesicular-extravesicular ratio of sucrose on PSD of

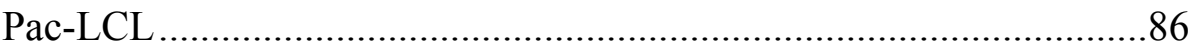

Effect of number of extrusion cycles on the PSD and PDI after lyophilization 87

Effect of primary drying temperature on PDI after lyophilization of Pac-LCL 
Figure 4-7.

Figure 4-8.

Figure 4-9.

Figure 4-10.

Figure 4-11.

Figure 4-12.

Figure 4-13.

Figure 4-14.

Figure 5-1.

Figure 5-2.

Figure 5-3.

Figure 5-4.

Figure 5-5.

Figure 5-6.

Figure 5-7.

Figure 5-8.

Figure 5-9.

Figure 5-10.

Figure 6-1.

Figure 6-2.

Figure 6-3.

Figure 6-4.

Figure 6-5.

Figure 6-6.

Figure 6-7.

Figure 6-8.
Effect of mannitol concentration on $\mathrm{Tg}^{\prime}$.... .90

Effect of mannitol concentration on the PSD and PDI after lyophilization 91

Effect of mannitol concentration on echogenicity of Pac-GFLCL ......92

In vitro drug leakage study of Pac-GFLCL ......................................94

Verification of conjugation efficiency of cRGD ..............................95

TEM images of Pac-GFLCL (A) and Pac-RGD-GFTLCL (B) ..........97

Disintegration of Pac-RGD-GFTLCL with ultrasound pulses ...........98

Stability of Pac-RGD-GFTLCL ................................................... 100

Stability data for Pac-RGD-TLCL dispersion ..................................112

Stability data for Pac-Tf-TLCL dispersion .....................................113

Representative NIRF images of localization of DiR formulations

in mice bearing MDA-MB-231 breast cancer

NIRF images of tissues isolated $72 \mathrm{~h}$ post injection showing the accumulation of DiR in tumor, liver, and spleen

Localization of DiR-loaded liposomal formulations in tumor tissue calculated from NIRF imaging ....

Localization of DiR formulations in the flank region to represent

the concentration in the circulation

Localization of DiR formulations in peritoneal cavity to

represent uptake in liver and spleen

Tumor/normal tissue ratio of DiR formulations calculated from

NIRF imaging

Biodistribution of paclitaxel formulations in plasma (A), and

tumor (B) of mouse xenografts bearing MDA-MB-231 breast

cancer.

Antitumor efficacy of paclitaxel formulations in mouse

xenografts bearing MDA-MB-231 breast cancer

Effect of sucrose concentration on $\mathrm{Tg}^{\prime}$.

Effect of sucrose concentration on the effect of paclitaxel

retention in the liposomes during lyophilization

Effect of sucrose concentration on $\mathrm{T}_{\mathrm{m}}$ of the lipids in the bilayer.....136

Effect of sucrose concentration on PSD of paclitaxel-loaded

liposomes during lyophilization.

Effect of internal-external ratio of sucrose on PSD during

lyophilization

Stability of lyophilized Pac-LCL formulation ................................141

Stability of lyophilized Pac-RGD-TLCL ....................................... 142

Stability of lyophilized Pac-Tf-TLCL 


\section{LIST OF ABBREVIATIONS}

$\begin{array}{ll}\Delta \mathrm{pH} & \text { Transmembrane pH gradient } \\ \Delta \psi & \text { Transmembrane potential gradient } \\ \text { ANOVA } & \text { Analysis of variance } \\ \text { BBB } & \text { Blood brain barrier } \\ \text { Biotinyl DSPE-PEG } 2000 & \begin{array}{l}\text { 1,2-distearoyl-sn-glycero-3-phoshoethanolamine-N- } \\ \text { (biotinyl poly(ethylene glycol)2000) }\end{array} \\ \text { BNCT } & \text { Boron neutron capture therapy } \\ \text { BSH } & \text { Mercaptoundecahydrododecaborate } \\ \text { cRGD } & \text { Cyclo (Arg-Gly-Asp-D-Phe-Cys) peptide } \\ \text { DiR } & \text { 1,1'-dioctadecyl-3,3,3'3'-tetramethylindotricarbocyanine } \\ & \text { iodide } \\ \text { DiR-LCL } & \text { DiR-loaded long-circulating liposomes } \\ \text { DiR-RGD-TLCL } & \text { DiR-loaded cRGD conjugated targeted long-circulating } \\ & \text { liposomes } \\ \text { D-MEM } & \text { Dulbecco's modified eagle medium } \\ \text { DOPE } & \text { Dioleyl phosphatidylethanolamine } \\ \text { DPPC } & \text { Dipalmitoyl phosphatidylcholine } \\ \text { DPPG } & \text { Dipalmitoyl phosphatidylglycerin } \\ \text { DSC } & \text { Differential scanning calorimetry } \\ \text { DSPE } & \text { Distearoyl phosphatidylethanolamine } \\ \text { DSPE-PEG } 2000 & \text { 1,2-distearoyl-sn-glycero-3-phoshoethanolamine-N- } \\ & \text { (poly(ethylene glycol)2000) } \\ \text { EDTA } & \text { Ethylenediaminetetraacetic acid } \\ \text { EFGR } & \text { Epidermal growth factor receptor } \\ \text { EPC } & \text { Egg phosphatidylcholine } \\ \text { EPR } & \text { Enhanced permeability and retention } \\ \text { ESR } & \text { Electron spin resonance } \\ \text { FTIR } & \text { Fourier transform infra red } \\ \text { GFLCL } & \text { Gas-filled long-circulating liposomes } \\ \text { GM1 } & \text { Monosialoganglioside } \\ \text { HEPES } & \text { N-2-hydroxyethylpiperazine-N'-2-ethanesulfonic acid } \\ \text { HER2 } & \text { Human epidermal growth factor receptor 2 } \\ \text { HPLC } & \text { High performance liquid chromatography } \\ \text { HSPC } & \text { Hydrogenated soy phosphatidylcholine } \\ \text { IL-2 } & \text { Interleukin 2 } \\ \text { LCL } & \text { Long-circulating liposomes } \\ \text { LUV } & \text { Large unilamellar vesicles } \\ \text { Maleimide DSPE-PEG } & 1,2-\text { distearoyl-sn-glycero-3-phoshoethanolamine-N- } \\ & \text { (maleimide poly(ethylene glycol)2000) } \\ \text { MDSC } & \text { Modulated differential scanning calorimetry } \\ \text { MGSV } & \text { Mean gray scale value } \\ \text { MLV } & \text { Multilamellar vesicles } \\ \text { MWCO } & \text { Molecular weight cut-off } \\ & \\ & \\ & \end{array}$




\begin{tabular}{|c|c|}
\hline NGPE & N-glutaryl-phosphatidylethanolamine \\
\hline NGR & Asn-Gly-Arg peptide \\
\hline NIRF & Near infrared fluorescence \\
\hline NMR & Nuclear magnetic resonance \\
\hline NS & Normal saline \\
\hline Pac-GFLCL & Paclitaxel-loaded gas-filled long-circulating liposomes \\
\hline Pac-LCL & Paclitaxel-loaded long-circulating liposomes \\
\hline Pac-RGD-GFTLCL & $\begin{array}{l}\text { Paclitaxel-loaded cRGD conjugated gas-filled targeted } \\
\text { long-circulating liposomes }\end{array}$ \\
\hline Pac-RGD-TLCL & $\begin{array}{l}\text { Paclitaxel-loaded cRGD conjugated targeted long- } \\
\text { circulating liposomes }\end{array}$ \\
\hline Pac-Tf-TLCL & $\begin{array}{l}\text { Paclitaxel-loaded transferrin conjugated targeted long- } \\
\text { circulating liposomes }\end{array}$ \\
\hline PDI & Polydispersity index \\
\hline $\mathrm{PE}$ & Phosphatidylethanolamine \\
\hline PEG & Polyethylene glycol \\
\hline P-gp & P-glycoprotein \\
\hline PS & Phosphatidylserine \\
\hline PSD & Particle size distribution \\
\hline PVA & Polyvinyl alcohol \\
\hline RES & Reticuloendothelial system \\
\hline RGD & Arg-Gly-Asp peptide \\
\hline RGD-GFTLCL & $\begin{array}{l}\text { cRGD conjugated gas-filled targeted long-circulating } \\
\text { liposomes }\end{array}$ \\
\hline RGD-TLCL & cRGD conjugated targeted long-circulating liposomes \\
\hline ROI & Region of interest \\
\hline RPHPLC & Reversed phase high performance liquid chromatography \\
\hline SALCL & Streptavidin conjugated long-circulating liposomes \\
\hline SUV & Small unilamellar vesicles \\
\hline $\mathrm{T}$ & Temperature \\
\hline TAT & trans-activating transcriptional activator \\
\hline TEM & Transmission electron microscopy \\
\hline $\mathrm{Tf}$ & Transferrin \\
\hline TfR1 & Transferrin receptor 1 \\
\hline $\mathrm{Tg}^{\prime}$ & $\begin{array}{l}\text { Glass transition temperature of maximally freeze } \\
\text { concentrated amorphous phase }\end{array}$ \\
\hline $\mathrm{T}_{\mathrm{m}}$ & Gel to liquid crystalline transition temperature \\
\hline $\mathrm{T}_{\mathrm{o}}$ & Transition temperature of the fully hydrated lipid \\
\hline VEGF & Vascular endothelial growth factor \\
\hline Z-Avg or Z-Average & Average diameter \\
\hline
\end{tabular}




\section{CHAPTER 1. INTRODUCTION}

\subsection{STATEMENT OF THE PROBLEM}

\subsubsection{Breast Cancer and Treatment Choices}

Cancer is the disease characterized by a rapid and abnormal proliferation of cells. Cancer cells originate from normal cells through a series of genetic changes leading to progressive conversion to cancer cells [1]. Cancer is a dreadful disease and is the second leading cause of death in United States [2,3]. About 1.5 million new cases of cancer and more than half-a-million deaths have been estimated in the United States for the year 2009 [4]. Solid tumors account for more than 85 percent of all the cases reported with cancer $[5,6]$. Breast cancer is the most commonly diagnosed form of solid tumors and is the second leading cause of death in western women. About 20-30\% women with breast cancers develop metastatic breast cancers. Breast cancer research has increased dramatically during the last 2 decades, resulting in an extraordinary progress in our understanding of the disease, and in more efficient, and less toxic treatment options. The treatment choices for breast cancer can be categorized into local or systemic therapy. Surgery, radiation therapy, hyperthermia, and photodynamic therapy are examples of localized therapy. Surgery is the treatment of choice for breast cancer which involves lumpectomy, partial mastectomy or quadrantectomy, and mastectomy. Radiation therapy is an adjuvant therapy in which high energy radiation is used to kill cancer cells remaining in the breast, chest wall, underarm area after the surgery. Local therapy is beneficial for non-metastatic forms of breast cancers whereas systemic therapy is required for metastatic forms of breast cancers. Systemic therapy by means of chemotherapy, hormonal therapy, immunotherapy or targeted therapy, and gene therapy involves administration of the respective therapeutic agent by the oral or parenteral route.

\subsubsection{Chemotherapy}

Chemotherapy is the use of drugs to kill cancer cells. It can be used as adjuvant therapy after surgery for patients with no evidence of metastasis. Adjuvant chemotherapy reduces the risk of recurrence of cancer. Chemotherapy can also be used as a neoadjuvant therapy to surgery wherein the drugs are administered before surgery to shrink the tumor so that it can be removed easily by surgery. Chemotherapy is the first line therapy for advanced breast cancer in which the cancer has already spread beyond the breast and underarm area at the time of diagnosis. In chemotherapy, the drug distributes throughout the body via blood stream to reach the cancer cells. This form of systemic therapy is very useful to kill breast cancer cells which have metastasized to distant organs. Apart from killing cancer cells, these drugs also kill normal cells and result in toxic effects. For chemotherapy to be effective, the drug should be delivered at the tumor sites at adequate concentrations [7]. Inadequate concentrations of the drug at the tumor microenvironment could result in regrowth of the tumor cells and development of resistance [5]. 


\subsubsection{Paclitaxel: Mechanisms of Action}

Paclitaxel, one of the most effective drugs used in chemotherapy of breast cancer, is a natural product isolated from bark of the Pacific Yew tree, Taxus brevifolia $[8,9]$. It exhibits cytotoxic activity by promoting the assembly of microtubules from tubulin dimers and stabilizes microtubules by preventing depolymerization. The stability results in inhibition of the normal dynamic reorganization of the microtubule network that is essential for vital interphase and mitotic cellular functions [9]. Paclitaxel, like other antimicrotubule agents, activates the intrinsic mitochondrial apoptotic pathway. Loss of the mitochondrial membrane potential by opening of the permeability transition pore results in the release of proapoptotic factor cytochrome c, caspase cascade activation, and DNA fragmentation [10-12]. These effects are modulated by the members of Bcl-2 family composed of proapoptotic proteins (Bax-like proteins and $\mathrm{BH} 3$-only proteins) and antiapoptotic proteins (Bcl-2-like proteins). Paclitaxel turns the balance of proapoptotic and antiapoptotic proteins towards apoptosis by translocation of Bax from cytosol to mitochondria and/or by phosphorylation mediated inhibition of Bcl-2 [13, 14].

\subsubsection{Drawbacks of Paclitaxel Therapy}

Paclitaxel is marketed as Taxol ${ }^{\circledR}$ and Abraxane ${ }^{\mathrm{TM}}$ as an adjuvant in the treatment of node-positive breast cancer or second line treatment of breast cancer after failure of combination chemotherapy for metastatic disease or relapse within 6 months of adjuvant chemotherapy. Due to poor solubility of paclitaxel, Taxol ${ }^{\circledR}$ was formulated with ethanol and Cremophor ${ }^{\mathbb{B}}$ EL. Although the product is diluted before use, the concentration of Cremophor ${ }^{\circledR} \mathrm{EL}$ is high enough to cause serious hypersensitivity reactions on administration requiring pre-medication with corticosteroids and antihistamines [15]. Abraxane $^{\mathrm{TM}}$, albumin-bound nanoparticle formulation of paclitaxel, addressed solvent related adverse effects of Taxol ${ }^{\circledR}$ and eliminated the need for premedication with corticosteroids. But, phase III clinical trials in metastatic breast cancer patients showed higher rates of sensory neuropathy compared to Taxol ${ }^{\circledR}$ treatment [16]. Apart from the toxicity associated with the formulation components, administration of free paclitaxel is also associated with systemic toxicities such as myelosuppression, and peripheral neuropathy [17]. So, it is imperative to deliver paclitaxel locally to the tumor sites to minimize systemic toxicity. Hence, there is a need for an effective drug delivery system for chemotherapy with paclitaxel to produce cytotoxic concentrations of the drug at the tumor sites without affecting normal tissues.

\subsubsection{Barriers in Drug Delivery to Solid Tumors}

Since cancer cells require nutrition to grow and metastasize in to other organs, they form new vessels, from existing vessels, which are structurally and functionally abnormal. These newly formed vessels are leaky, tortuous, dilated, and saccular and have abnormal interconnections [6]. The variations in distribution of blood vessels in a solid tumor can range from well perfused periphery to seminecrotic to avascular or necrotic 
regions at the core of the tumor. In addition, hydrostatic pressure generated by proliferating cancer cells within the tumor can compress the blood vessels and lymphatic vessels leading to impaired blood flow and lymphatic drainage. The high interstitial pressure in tumors compared to normal tissues results in a net convection flow in the tumor interstitium outwards from the core of the tumor. Conventional and modern therapeutic agents for solid tumors include molecules, particles, and cells. To reach cancer cells in a solid tumor, these agents must make their way to the blood vessels of a tumor, cross the vessel wall into the interstitium, and then transport through the interstitial matrix $[5,7]$. The mechanisms of transport involve diffusion and/or convection depending upon the size, charge, and configuration of the agent [7]. Transport of small molecules in the interstitial space is mainly by diffusion which is governed by diffusivity and the concentration gradient. The transport of larger molecules is by convection which depends on hydraulic conductivity and pressure difference [5, 18]. The unique nature of solid tumors with high interstitial pressure, and heterogeneity in vasculature pose a formidable barrier to delivery of therapeutic agents [19].

\subsubsection{Strategies for Effective Delivery of Paclitaxel to Solid Tumors}

An ideal drug delivery system should improve the therapeutic efficacy of paclitaxel overcoming the barriers to drug delivery and also minimize toxicity, and lower the total dose required for the therapy. Targeted drug delivery fulfils many of these desirable attributes, improving efficacy of many existing drugs by altering pharmacokinetics and biodistribution [20]. Drug targeting can be broadly classified into passive targeting and active targeting. Passive targeting is the accumulation of therapeutic agent at the target site due physicochemical nature of the carrier or due to pathological factors of the target site. In contrast, active targeting involves the attachment of specific active ligands to the surface of the carrier so that it can recognize target receptors expressed on the surface of specific cell, tissue, or an organ. Tumor cells express many molecules on their surface which is not expressed by normal cells. Some receptors show higher expression in the tumor cells as compared to normal cells. So, solid tumors stands out as one disease that will get most of the benefits of targeted drug delivery [21]. Nanoscaled delivery systems are of particular interest in targeted drug delivery to solid tumors because of their ability to escape clearance by reticuloendothelial system (RES). The clearance of nanoparticulate systems by the RES increases with an increase in size. Nanoscaled systems approved or under clinical trial for use in drug delivery include liposomes, albumin based particles, PEGylated proteins, biodegradable polymer-drug composites, polymeric micelles, polymer-drug-conjugate based particles, dendrimers, and inorganic or solid particles [22].

\subsubsection{Liposomes for Paclitaxel Delivery to Solid Tumors}

Liposomes are biocompatible carriers with ability to incorporate both lipophilic and hydrophilic drugs [23]. The entrapped drug is protected from inactivation, and free drug is not available in the circulation which, in turn, minimizes systemic toxicity of the drug. 
Therefore, preparation of liposomes of paclitaxel can alter the pharmacokinetic and pharmacodynamic properties of the free drug and result in reduced toxicity and enhanced efficacy of the treatment. In addition, flexibility in design of liposomes allows selective localization of these carriers by passive targeting due to enhanced penetration and retention (EPR) effect at the tumor sites, or by active targeting due to targeting ligands conjugated to the surface of the liposomes [24-27]. An ideal carrier for drug delivery must have a longer circulation time which can be achieved by reducing the size of liposomes and/or modifying the surface with poly(ethylene glycol) derivatives [28]. Targeting capability can be achieved by attaching specific ligands onto the surface of the liposomes. Thus, incorporation of paclitaxel in the bilayer of targeted long-circulating liposomes not only deliver the drug specifically to the tumor cells but also minimize systemic toxicity associated with free drug, by minimizing the exposure to normal tissues. The release of drug from these liposomes, at the target site, is determined by one or more of the following natural cytosolic mechanisms [23].

- Specific or non-specific adsorption on to the cell surface

- Fusion with the cell membranes and subsequent release of the drug into the cytoplasm

- Adsorption onto the cell surface resulting in destabilization of cell membrane components so that the released drug can enter via micropinocytosis

- Direct or transfer protein mediated exchange of lipid components with cell membrane

- Specific or non-specific endocytosis in which the endosomes deliver the drug into the lysosome or the endosomes undergo destabilization and release the drug in cytoplasm

\subsubsection{Stability of Liposomes}

The liposomal encapsulated drug tends to leak from the liposomes on storage of these vesicles in aqueous dispersions. In addition, the liposomes can fuse together and form larger liposomes and result in an increase in vesicle size distribution. The stability of liposomes can be improved by freezing, lyophilization, spray drying, and supercritical fluid technology [29-31]. Lyophilization stands out as a preferred method to improve long-term shelf life of liposomes especially those containing thermosensitive drugs [32]. By removing water from the liposomal dispersion by lyophilization, the structural and permeability properties can be retained [33]. However, the lyophilization process itself can induce physical changes such as loss of the encapsulated agent, and an increase in vesicle size [34]. Careful selection and optimization of the formulation and process is necessary to improve the shelf life of paclitaxel-loaded liposomes.

\subsubsection{Echogenic Liposomes}

Apart from targeting and controlled release, it may be an additional advantage to improve permeability at the tumor vasculature. Incorporation of gases in the bilayer of 
liposomes makes them ultrasound active [35]. These echogenic liposomes have the potential for stress on activation by ultrasound and eventually release the contents at the target site. These liposomes can be visualized at the target site, and by changing the intensity of ultrasound, they can be disintegrated providing a means for controlling the drug release. Interestingly, the disintegration of gas-filled species by contrast enhanced ultrasound produces liquid jets that can produce small gaps in the vasculature and improve the permeability [36]. This phenomenon exhibited by microbubbles can be explored for targeted delivery using echogenic liposomes wherein the targeted liposomes maintain basal levels of the drug by virtue of cytosolic transport, and by altering intensity of ultrasound, high cytotoxic concentrations of the drug can be achieved at the target site. Thus, cavitation effect of ultrasound on the echogenic liposomes provides a control over drug release, and also enhances drug permeability by producing small gaps in the endothelial walls of vasculature [36-39].

\subsection{REVIEW OF LITERATURE: FORMULATION DEVELOPMENT, AND DELIVERY ASPECTS OF LIPOSOMES IN CANCER CHEMOTHERAPY}

Liposomes are bilayered vesicles made of phospholipids. In 1964, Bangham and Horne described that lecithin dispersions contained 'spherulites' composed of concentric lamellae by negative staining technique using electron microscope [40]. This liquid crystal phase structure of phospholipids in water or salt solutions was referred as 'smectic mesophase'. These lipid systems were tested for the permeability of anions and cations through the membrane to describe their analogy to certain biological membranes [41, 42]. These artificial structures were colloquially referred as 'Liposomes' or 'Bangasomes' $[43,44]$. Later, the term liposomes, derived from greek words 'lipos' meaning fat and 'somas' meaning body, gained popularity in literature and was generally accepted. Liposomes were used initially as model membranes to characterize the behavior of cell membrane. Gregordias and coworkers explored the therapeutic potential of liposomes by developing Aspergillus niger aminoglucosidase entrapped liposomes as enzyme carriers in enzyme replacement therapy of glycogen storage diseases $[45,46]$. These radio labeled liposomes were removed rapidly from plasma following intravenous administration to rats. About $56 \%$ of the total radioactivity was recovered in liver within $15 \mathrm{~min}$ of administration. These results showed that liposomes can be used as effective carriers for delivery of enzymes to liver and spleen. Further, they developed liposomes containing invertase ( $\beta$-D-fructofuranosidase) to follow the fate of enzyme activity rather than following radioactivity of labeled liposomes. These liposomes accumulated in liver and spleen within 6 hours of intravenous injection in rats. The invertase activity was maximum in the mitochondrial-lysosomal fraction of the liver suggesting that the liposomes can be used as potential carriers for delivering enzymes and drugs to liver and spleen $[47,48]$. Then, they tested the applicability of liposomes in drug delivery by developing liposomes loaded with actinomycin $\mathrm{D}$ in the lipid bilayer or penicillin in the aqueous core of the liposomes [49]. The distribution of liposome entrapped, and nonentrapped drug was studied by intravenous injection to rats. Though the elimination of liposomal entrapped drugs was slower than the free drugs, a considerable amount of the liposomal drug accumulated in lysosomes of liver and spleen. On reaching lysosomes, the 
drug action requires disruption of liposomes by lysosomal lipases to release the drug. As a further development, they evaluated the uptake of liposome entrapped agents in the tumor cells using radio labeled albumin entrapped liposomes [50]. The study involving three metastatic cancer patients showed that the radioactivity in the tumors was slightly higher to much higher than that of the corresponding normal tissues. The improved localization was attributed to extensive vascularization in tumor and enhanced endocytotic activity of the tumor cells.

From the time liposomes found their way in drug delivery, extensive research in the area lead to numerous advances to improve the formulation and to improve their efficiency as a carrier in drug delivery [28, 51-54]. The following sub-sections will briefly outline basic properties, and some of the advances in formulation development of liposomes with special emphasis in their application in chemotherapy of solid tumors.

\subsubsection{Composition of Liposomes}

Liposomes are composed of natural or synthetic phospholipids. Phospholipids have a head group and a tail group with two fatty acyl chains of varying lengths. The phosphate head group is attached to different functional groups which can determine the degree of hydration and charge of the liposomes [28]. The choline functional group offers some degree of hydration and confers a neutral charge. Ethanolamine contributes to a minimum degree of surface hydration while it is also a neutral in charge. On the other hand, serine and glycerin functional groups confer a negative charge with some surface hydration. Polyethyleneglycol (PEG) ethanolamine provides high degree of hydration and a steric effect with a negative charge. The properties of tail group fatty acyl chains determine fluidity, thickness, and order of membrane packing of the bilayer. The chain length can range from $\mathrm{C}_{14}-\mathrm{C}_{18}$. An increase in chain length increases the thickness, and gel to liquid crystalline transition temperature $\left(\mathrm{T}_{\mathrm{m}}\right)$ of the bilayer. An increase in the level of saturation increases the rigidity and $\mathrm{T}_{\mathrm{m}}$, and reduces fluidity of the bilayer. Varying the degree of saturation and chain length of the two tail acyl chains can result in a decrease in the order of membrane packing and a lower $\mathrm{T}_{\mathrm{m}}$ compared to lipids with two identical acyl chains [55].

\subsubsection{Effect of Fluidity of the Bilayer}

The lipid bilayers of liposome membranes exist in the well ordered gel phase at temperatures below their gel to liquid crystalline transition temperature $\left(T_{m}\right)$. The $T_{m}$ involves partial melting of acyl chains while the spherical liposomes remain intact. At temperatures above $T_{m}$, the lipids exist in a disordered fluid phase or liquid crystalline phase wherein the acyl chains become mobile within the hydrophobic region of the liposome while the anchoring of polar head groups prevent complete freedom of movement $[28,56]$. At the temperatures corresponding to $\mathrm{T}_{\mathrm{m}}$ of the phospholipids, maximum permeability or leakage is observed [57]. The phase behavior of liposomes determines their permeability, aggregation, ability to bind to serum proteins, and fusion 
[28]. The phase behavior on the other hand, depends on the composition of the bilayer. By careful selection of chain length, saturation/unsaturation of fatty acyl chains of the lipids, the fluidity of the bilayer can be altered.

Introduction of double bonds and shortening the chain length of phospholipids can increase the fluidity of the bilayer and thereby increase the permeability across the bilayer [55]. The permeability of glycerol and erythritol was evaluated in liposomes made of different phospholipids. The swelling rates of liposomes composed of (distearoyl)lecithin or (1-stearoyl-2-oleyl)lecithin or (dioleyl)lecithin or (dilinoleyl)lecithin showed that the permeability increased with an increase in number of double bonds. Similarly, liposomes made of (disteroyl)lecithin or (dipalimitoyl)lecithin or (dimyristoyl)lecithin showed a decrease in permeability with an increase in chain length as glycerol could not to penetrate the bilayer with increasing chain length.

Thermal motion of fatty acyl chains result in molecular cavities. When cholesterol is added along with phospholipids, it occupies these cavities and therefore, does not increase area per molecule in mixed monolayers. This observation was proved by the measurement of surface potential for palmitoyl lecithin-cholesterol mixtures which did not show a change in surface potential [58]. At high surface pressures cholesterol liquefied solid monolayers indicating that cholesterol imparts fluidity to the phospholipid monolayers. Ladbrooke et al showed that cholesterol controls fluidity of the hydrocarbon chains of phospholipids by disrupting the crystalline chain lattice of the gel phase, and by inhibiting the flexibility of chains in the dispersed liquid crystalline phase [59]. For lipids which would normally be above their $\mathrm{T}_{\mathrm{m}}\left(\mathrm{T}>\mathrm{T}_{\mathrm{m}}\right)$, there will be a limitation in flexibility and for those lipids which remain at gel state $\left(T<\mathrm{T}_{\mathrm{m}}\right)$ have greater fluidity.

Liposomes containing mixtures of phospholipids and cholesterol demonstrated a decrease in permeability with an increase in concentration of cholesterol [55]. Cholesterol reduced the permeability of glycerol in liposome structures made of egg-yolk lecithin or (1-palmitoyl-2-oleyl)-lecithin or (dioleyl) lecithin in a concentration dependant manner. The effect of cholesterol permeability was not limited to glycerol. A decreased chloride permeability, from liposomes made of egg-yolk lecithin and cholesterol compared to that pure lecithin, has been reported [60]. Valinomycin induced permeability of ${ }^{86} \mathrm{Rb}^{+}$was also reduced by inclusion of cholesterol in the liposome composition [61]. Demel et al explained that the permeability reducing property of cholesterol is related to planar sterol nucleus, an intact side chain, and a $3 \beta$-hydroxyl group based on their study with several natural and synthetic sterols on the permeability of glucose, glycerol, and ${ }^{86} \mathrm{Rb}^{+}$using egg lecithin liposomes [62]. The reduced permeability is due to increased packing and decreased mobility of the hydrocarbon chains [63].

Kirby et al demonstrated that the inclusion of cholesterol in the liposomes improves in vitro and in vivo stability of the liposomes using a fluorescent tracer carboxyfluorescein [64]. The liposomes rich in cholesterol (1:1 ratio of egg phosphatidylcholine (EPC) and cholesterol) were more stable following intravenous injection to mice compared to liposomes containing less or no cholesterol. A similar stability trend was observed in mouse whole blood, plasma or serum. The stabilizing 
effect was similar in neutral, negative, and positive charged liposomes. The liposomes containing cholesterol were resistant to exchange of phospholipids with high density lipoproteins and thereby exhibited better in vivo stability.

\subsubsection{Effect of Surface Charge of the Liposomes}

The liposomes have a charge based on the composition of head group of the phospholipids. These phospholipids can have neutral, positive, or negative charge. The surface charge of the liposomes determines their stability in dispersion, in vivo circulation time, ability to interact with target cells, and recognition by plasma proteins or opsonins. Neutral liposomes have lower tendency to be cleared by the RES but have poor stability in dispersion due to aggregation. Negative surface charge provides better colloidal stability, but these liposomes have higher non-specific uptake in vivo, and are easily recognized by macrophages. With permeability of the bilayer remaining constant between neutral and negatively charged liposomal formulations, negatively charged liposomes were cleared faster than the liposomes with neutral charge [65]. Liposomes with different surface charge were removed from circulation at different rates even though the total body clearance of encapsulated compound is similar [66]. Hepatic uptake of positive and negatively charged liposomes was greater than the liposomes of neutral charge [67]. Neutral liposomes gain negative charge in presence of blood plasma due to adsorption or binding plasma components whereas negatively charged liposomes remain unchanged [68]. Wilkins and myers suggested that surface charge of the particle could affect the steric arrangement of the adsorbed protein in a manner that is recognizable by the fixed macrophages of the RES [69].

Phagocytosis of complement opsonized liposomes was strongly dependent on the charge of the liposomal lipids. The presence of acidic negatively charged lipids phosphatidylserine, phosphatidylinositol and dicetyl phosphate profoundly suppressed the uptake of the liposomes [70]. The drug penetration through the skin was greater from a gel formulation containing negatively charged liposomes compared to positively charged liposomes [71]. Histological studies showed that negatively charged liposomes diffused to the dermis and lower portions of hair follicles through the stratum corneum and the follicles much faster than the positively charged vesicles. Electrostatic interactions are one of the main forces affecting the association of liposomes with the charged cell surface. An increase in the concentration of negatively charged lipid phosphatidylserine (PS) in the lipid bilayer resulted in increased association of liposomes with human colon CX-1.1 cancer cells [72]. Heath and coworkers demonstrated that the uptake of methotrexate- $\gamma$-aspartate from negatively charged liposomes was more efficient in five different cancer cell lines as compared to neutral liposomes [73].

Positively charged liposomes, frequently used for condensation of DNA on the surface for gene delivery, also have shown higher clearance by the RES. The degree of interaction of liposomes with macrophages can be modulated by the positive charge on the liposomes [74]. The ${ }^{99} \mathrm{mTc}$ marker entrapped in positively charged liposomes was 
cleared five times faster than the lipid integral membrane marker, showing that the presence of positive charge affects in vivo stability of liposomes [75].

In vitro cellular binding and uptake study using human ovarian carcinoma HeLa cell lines showed that endocytosis of positively charged liposomes was greater than negatively charged liposomes, and neutral liposomes [76]. Cationic liposomes have shown the tendency to preferentially accumulate the A-Mel-3 melanoma of the hamster in comparison to normal surrounding tissue [77]. Intravital microscopy revealed that the uptake of cationic liposomes in the solid tumor was due to their accumulation in angiogenic tumor vessels but neutral, and negatively charged liposomes showed nonspecific extravasation into parenchyma. Positively charged paclitaxel-loaded liposomes showed a significant regression of tumor and delayed metastasis due to antivascular targeting by virtue of positive charge $[77,78]$.

\subsubsection{Size of Liposomes}

The size of the liposomes is one of the major factors determining their fate after intravenous injection [79]. Larger liposomes are cleared rapidly from the circulation following intravenous administration. These liposomes are taken up into Kupffer cells of liver and fixed macrophages of spleen [80]. Smaller liposomes of size less than $100 \mathrm{~nm}$ have the ability to pass through fenestrated endothelium, or regions of increased capillary permeability, and gain access to liver parenchymal cells. Liver and spleen are the major sites of localization for liposomes irrespective of their size. Harashima and Kiwada compiled the data from literature for pharmacokinetics of liposomes of different sizes and calculated the total body clearance [79]. Their analysis indicated that the clearance was proportional to the size of the liposomes. AmBisome ${ }^{\mathrm{TM}}$ (Gilead Sciences, Inc., Foster City, CA), the marketed liposomal formulation of amphotericin B is formulated to the size specification of 45-80 $\mathrm{nm}$ to reduce RES uptake [28].

Liposomes of different size were treated with serum and their uptake was determined in perfused liver [81]. The results showed that opsonins bind to the liposomes depending upon the size, and they were taken up by the liver phagocytosis based on the extent of opsonization. The hepatic extraction of liposomes increased with an increase in liposome size (200, 400 and $800 \mathrm{~nm})$. Allen and Everest studied the effect of liposome size and composition on pharmacokinetics of encapsulated $\left[{ }^{14} \mathrm{C}\right]$ labeled sucrose [82]. They used two liposomal compositions egg phosphatidylcholine-cholesterol, and sphingomyelinphosphatidylcholine with in vitro serum stability of 2.5 and $35 \mathrm{~h}$ respectively. Liposome entrapment increased the circulation time of sucrose in vivo with a higher circulation time for small unilamellar vesicles compared to multilamellar vesicles. The same trend of in vitro stability was observed in vivo. Senior et al showed with liposomes of similar composition, the trend is that increase in size results in rapid clearance [65]. The effect of size was prominent in determining the circulation half life of liposomes than the membrane permeability. 
The kinetics of differential uptake of various types of liposomes by the liver Kupffer cells and parenchymal cells were evaluated by Rahman and coworkers [83]. Liposomes of different size were loaded with ${ }^{59} \mathrm{Fe}$-Desferal. The uptake in two cell types of liver was monitored at specific time points following intravenous injection to mice. Fractionation of the liver into parenchymal and non-parenchymal cell fractions revealed that liposomes of size $60-80 \mathrm{~nm}$ were taken up by the parenchymal cells, while the larger multilamellar liposomes of size $500 \mathrm{~nm}$ were taken up by Kupffer cells. These results suggested that the size plays an important role in sorting liposomes to Kupffer cells or hepatocytes.

The activation of complement system can also induce complement mediated clearance of liposomes [81]. When inactivated serum was used to omit the activity of opsonins, size dependant degradation in serum was observed which was consistent with size dependant complement activation. The uptake of these liposomes in inactivated serum by the liver was primarily due to complement activation. Abra et al quantified the relationship between in vivo disposition and dose size for liposomes of three different diameters $(460,160,58 \mathrm{~nm})$ in mice [84]. Saturation in liver with increasing lipid dose was seen with all three sizes together with increase in blood levels.

\subsubsection{Surface Modification of Liposomes}

The clearance of liposomes by RES depends on non-specific hydrophobic interactions of liposomes with RES cells, and specific opsonization reaction involving blood components. The liposomes cleared by RES accumulate into Kupffer cells of liver or fixed macrophages of spleen. This will be an advantage if the liposomes are targeted to the organs of RES. But, delivering the liposomes to non RES organs and tissues require approaches to overcome the short circulation time due to clearance by RES. In addition, repeated administration followed by accumulation of liposomes in the RES organs can impair the RES and result in consequences of altering the host defense mechanism.

The rate of clearance of intravenously administered liposomes depends on several factors including surface charge. Even though, liposomal membranes are similar to cell membranes they are cleared rapidly by several mechanisms. But, analysis of composition of cell membranes revealed that the presence of large amounts of sialic acid residues on the cell membranes can impart negative charge to the surface and thereby improved stability. Allen et al. developed liposomes with lipid composition which mimics surface characteristics of erythrocytes by including ganglioside $\mathrm{G}_{\mathrm{M} 1}$ in the formulation $[80,85]$. Biodistribution studies in mice showed that the liposomes containing ganglioside $\mathrm{G}_{\mathrm{M} 1}$ have longer circulation time compared to conventional liposomes, and other negatively charged liposomes containing dipalmitoylphosphatidylglycerin (DPPG), and sulfatides. The blood/RES ratio for the ganglioside $\mathrm{G}_{\mathrm{M} 1}$ containing liposomes increased in the presence of bilayer rigidifying lipid, sphingomyelin. The data demonstrated that surface sialic acid and bilayer viscosity worked synergistically increasing circulation time of liposomes. The authors also evaluated the effect of size on biodistribution of liposomes of various compositions in the presence and absence of ganglioside $\mathrm{G}_{\mathrm{M} 1}$. Circulation halflives increased with decreasing size down to liposomes extruded through $80 \mathrm{~nm}$ filters. 
Further decrease in size resulted in an increased accumulation in liver possibly due to the ability of the smaller liposomes to penetrate liver sinusoids and gain access to hepatocytes. Gabizon and Papahadjopoulos [86] confirmed the improved circulation time, and reduced clearance of $\mathrm{G}_{\mathrm{M} 1}$ containing liposomes. Further, they demonstrated a 25-fold increase in tumor uptake of the liposomal tracer in a J6456 lymphoma muscular tumor model in mice as compared to the free tracer. In another study, they showed that physicochemical features contributing to optimal retardation of liposome clearance include a hydrophilic carbohydrate moiety and a sterically hindered negatively-charged group [87]. Asialoganglioside (AGM1) was less effective in retarding clearance of liposomes than monosialoganglioside (GM1) indicating that there is a need for negative charge in addition to the carbohydrate group. The negatively charged lipids phosphatidylinositol phosphate (PIP) and trisialoganglioside (GT1) were less effective than phosphatidylinositol (PI) and GM1 in retarding clearance of liposomes. Increasing the molar fraction of negatively charged lipid resulted in an increased uptake in the liver.

Blume and Cevc [88] demonstrated that the steric surface protection offered by PEG chains slows down the adsorption of macromolecules from blood on to the vesicles, and thereby, suppresses the clearance of the liposomes by RES. In vivo evaluation in mice showed that the steric barriers prolonged the vesicle circulation time for up to $24 \mathrm{~h}$. The authors claimed that the polymeric coating with PEG is non-immunogenic, and non-toxic as against the inclusion of natural glycolipids. Although glycolipids are nonimmunogenic, their use in artificial membranes at higher concentrations, not encountered in normal membranes, can trigger immunogenic reaction. In a similar direction, Klibanov et al demonstrated that coating the surface of the liposomes with PEG improves circulation time of the liposomes in blood minimizing the uptake by the RES [89]. In a biodistribution study in mice, the authors showed that the circulation half-life of PEG coated liposomes were 10-fold greater than conventional liposomes of same size. Coating the liposomes with PEG increases the hydrophilicity of the liposome surface and minimizes the hydrophobic interaction with RES. It also sterically prevents the binding of opsonins to the liposomes to overcome specific interaction with RES. Doxil ${ }^{\circledR}$ (Alza Corporation, now part of Johnson \& Johnson, Inc., New Brunswick, NJ), the marketed liposomal formulation of doxorubicin is a PEGylated formulation to overcome clearance by the RES.

Liposome grafted PEG can neutralize the surface charge of the liposomes and provide shielding from interaction with various opsonins. The effect of PEG in minimizing the clearance is dependent upon the charge of the liposomes and the chain length of the surface grafted PEG polymer [90]. The clearance of positively charged liposomes was minimized by incorporation of either phosphatidylethanolamine (PE) conjugated to $\mathrm{PEG}_{750}$ or $\mathrm{PEG}_{5000}$ whereas the clearance of negatively charged liposomes was minimized only by PE-PEG 5000 but not PE-PEG 750 . The presence of negatively charged lipid phosphatidylserine (PS) on the membrane surface can form transient nucleation sites for binding plasma proteins which in turn increases the clearance. These nucleation sites may not be accessible when PEG-lipids are present at elevated levels where they adopt a highly compressed brush conformation. An increase in the concentration of distearoylphosphatidylethanolamine- $\mathrm{PEG}_{2000}\left(\mathrm{DSPE}-\mathrm{PEG}_{2000}\right)$ showed a decrease in 
clearance of these negatively charged liposomes with $15 \%$ providing almost complete shielding effect [91]. The combined effects of densely packed PEG and extension of the polymer barrier from the membrane surface provided steric inhibition of high affinity protein-membrane interactions.

The presence of modified polyvinyl alcohol (PVA) on the surface of liposomes can affect the interaction of liposomes with serum proteins inhibiting the protein adsorption [92]. Flow cytometry and confocal laser scanning microscopy evaluation of interaction of liposomes containing DiI (1,1'-dioctadecyl-3,3,3',3'-tetramethylindocarbocianin perchlorate) with $\mathrm{J} 774$ cells revealed that there is a steric hindrance effect on the interaction [93]. The introduction of a PVA layer on the liposomal surface inhibited the interaction of the liposomal core and J774 cells in a similar fashion as with PEG-coated liposomes. The use of a mixture of PEG and PVA in the surface modification has been shown to prolong the residence time of the liposomes compared to those with PEG alone [94]. A decrease in the adsorbed amount of several opsonins and an increase in the adsorbed amount of dysopsonins were responsible for lower affinity of these liposomes to the liver and longer residence in the systemic circulation.

\subsubsection{Drug Loading in Liposomes}

The unique nature of the liposomes is that their ability to incorporate lipophilic drugs in the bilayer, and hydrophilic drugs in the aqueous core. The flexibility in the composition of bilayer allows modification of bilayer integrity and fluidity to prepare stable drug-loaded liposomes. It is also possible to load both lipophilic and hydrophilic drugs in the liposomes. Drug loading in the liposomes can be accomplished by passive loading and active loading and is reviewed by Mayer and coworkers [95]. Drug loading efficiency can vary with the nature of the drug, lipid composition, type of liposomes, size, drug-lipid ratio, and method of preparation.

\subsubsection{Passive Loading}

For hydrophilic drugs, passive loading refers to the ability of the liposomes to capture certain aqueous volume along with dissolved drug in the core of liposomes during vesicle formation. The loading efficiency will vary with the type of liposomes as small unilamellar vesicles (SUV) have a lower trapped volume as compared to large unilamellar vesicles (LUV) and multilamellar vesicles (MLV).

Methotrexate was loaded in the aqueous core of liposomes by passive loading to encapsulate the drug for delivery to liver cancer [96]. Due to small trapped volume, about $18 \%$ of the drug was entrapped in liposomes. Large unilamellar liposomes prepared by extrusion procedure with $33 \%$ loading efficiency for methotrexate and cytosine arabinoside have been reported [97]. Szoka and Papahadjopoulos developed large unilamellar and oligolamellar liposomes with high trapped volumes by reversed phase evaporation technique to encapsulate up to $65 \%$ of aqueous phase [98]. They improved 
the loading of cytosine arabinoside by inverted micelle formation within organic solvent followed by solvent removal to improve encapsulation.

\subsubsection{Active Loading}

Active loading refers to entrapment of lipid soluble or water soluble drugs in the bilayer or aqueous core of liposomes respectively due to nature of the drug and lipids. Lipid soluble drugs are dissolved along with phospholipids during film formation step in the preparation of liposomes. The entrapment of these drugs is dependent on packing restrictions of the bilayer and can vary with lipid composition. On the other hand, amphiphilic drugs are often difficult to entrap in the liposomes because they easily permeate through the bilayer. The interaction between the drug and charged head group of the lipid components has been explored to improve loading efficiency. Electrostatic interaction and/or intercalation of doxorubicin with negatively charged PS or cardiolipin in the bilayer improved the drug loading efficiency as compared to neutral liposomes [99$101]$.

Amphiphilic cations can be effectively loaded into the aqueous core liposomes through an ion gradient across the membrane. Cationic anticancer drugs doxorubicin, and vinblastine were actively loaded into the liposomes when a membrane potential $(\Delta \psi$, negative inside) is generated across the vesicle membrane [97]. Membrane potentials were generated by forming liposomes in potassium glutamate buffer followed by exchange of unentrapped buffer with sodium chloride buffer using Sephadex G-50 desalting columns. Addition of $\mathrm{K}^{+}$ionophore, valinomycin, induced $\mathrm{K}^{+}$diffusion potential and improved the drug loading into the aqueous core of the vesicles when the drug was added. In this active loading technique, the retention time of doxorubicin was improved by an order of magnitude compared to liposomes loaded with passive trapping technique. Another strategy of active loading involves the use of transmembrane $\mathrm{pH}$ gradients $(\Delta \mathrm{pH}$, acidic inside). In this case, the liposomes were hydrated in presence of an acidic buffer ( $\mathrm{pH} 4.6$ ), unentrapped acidic buffer was exchanged with a buffer of higher $\mathrm{pH}(\mathrm{pH} 7.5)$ to generate a transmembrane $\mathrm{pH}$ gradient. Addition of doxorubicin results in active loading due to the $\mathrm{pH}$ gradient. The loading and retention of doxorubicin was several folds higher than the liposomes with passive loading [102]. Although both techniques employing $\Delta \psi$, and $\Delta \mathrm{pH}$ improve drug loading efficiency, the use of $\Delta \mathrm{pH}$ will be of practical significance because the techniques with $\Delta \psi$ require the presence of external ionophore but the techniques with $\Delta \mathrm{pH}$ does not require any additives [95]. Since the drugs are loaded in to preformed liposomes in these remote loading techniques, harsh conditions of processing is avoided which is beneficial for labile drugs.

Another remote loading approach uses ammonium sulfate gradient where the concentration of ammonium sulfate in the aqueous core is greater than the medium in which liposomes are suspended. The gradient of liposomal ammonium sulfate concentration to the concentration in the medium acts as a driving force and works by base exchange of ammonium ions for loading amphipathic weak bases [103]. Doxorubicin was loaded by ammonium sulfate gradient method into liposomes such that 
the concentration of doxorubicin in the aqueous core was more than 100-fold than the medium. In addition to $\mathrm{pH}$ gradient and ammonium ion diffusion potential, low permeability coefficient of sulfate ions through the bilayers is also crucial in stable accumulation. The low solubility of anthracycline sulfate salt formed in the aqueous core is further lowered by the presence of ammonium sulfate. Salting out effect of ammonium sulfate accelerates the flocculation and gelation of doxorubicin and improves the encapsulation and stability.

\subsubsection{Coencapsulation}

Almost all chemotherapeutic regimens for the treatment of cancer include combination of drugs. Administration of combination drugs loaded in liposomes will be less prone to development of drug resistance compared to therapies with single drug or drugs administered sequentially. Combining liposomal formulations of two drugs will have adverse effects associated with high lipids and hence coencapsulation of the combination drugs is sought. However, there are difficulties associated with efficient and stable encapsulation of two chemically different drugs. Methotrexate or cytosine arabinoside were loaded passively into the aqueous core of liposomes followed by active loading of doxorubicin through valinomycin induced $\mathrm{K}^{+}$membrane potential [97]. Tardi et al showed that it is also necessary to maintain a constant ratio between two drugs to benefit from synergy of the treatment [104]. They developed liposomes containing irinotecan and floxuridine in the aqueous core of the liposomes. These drugs were loaded into the liposomes by simultaneous drug loading wherein floxuridine was passively loaded, and irinotecan was actively loaded in to the liposomes in presence of copper gluconate/triethylamine buffer. Biodistribution study in mice showed that the optimized formulation maintained irinotecan-floxuridine ratio of $1: 1$.

Doxorubicin and combretastatin A4 were coencapsulated in Arg-Gly-Asp (RGD) peptide conjugated liposomes for targeted delivery to melanoma [105]. Combretastatin was loaded in the bilayer during the film formation step and doxorubicin was remote loaded in to the aqueous core of liposomes by ammonium sulfate gradient method. RGD conjugated formulation showed greater efficacy in regressing tumor volume in B16F10 flank tumor melanoma xenograft model in C57BL/6 mice. The authors proposed that combretastatin disrupts the vascular matrix leaving the encapsulated gel like doxorubicin in the tumor site, and doxorubicin is released subsequently to exert cytotoxic effect

The use of modulators to overcome drug resistance is a topic of interest in coencapsulation of liposomes. Calcium $\left(\mathrm{Ca}^{2+}\right)$ channel blockers inhibit the tumor proliferation by inhibiting $\mathrm{Ca}^{2+}$ influx and evoke passive depletion of internal $\mathrm{Ca}^{2+}$ stores. Amlodipine was coencapsulated as a modulator for topotecan to overcome multi drug resistance in leukemia [106]. Both drugs were loaded in the aqueous core by ammonium sulfate gradient method. The results from in vitro apoptosis study in non resistant K562 and resistant MDR HL-60 cell lines showed that amlodipine induces apoptosis by activation of caspase $3 / 7$ and 8 activities. Depletion of internal $\mathrm{Ca}^{2+}$ stores by amlodipine lead to activation of caspase 8 , then in the cascade, activation of other effector caspases 3 
and 7. In vivo evaluation in female nude mice xenografts bearing MDR HL-60 tumor showed that the stealth liposomes containing topotecan and amlodipine showed a significantly lower change ratio for the tumor size compared to free topotecan, and stealth liposomal topotecan. The combination therapy worked by potentiating the apoptotic effect and reversing the resistance.

\subsubsection{Targeted Drug Delivery with Liposomes}

The drugs used in cancer chemotherapy often have severe adverse effects because they not only kill cancer cells but also kill normal cells. Encapsulation of these drugs in the liposomes can minimize systemic exposure of the drug, and therefore, minimize undesired systemic toxicity. Further, selective localization of the drug at the tumor sites would reduce the total amount of drug required for the therapy. Thus, an ideal liposomal drug delivery system for cancer chemotherapy should improve localization of the drug at the tumor sites and minimize exposure to normal tissues. Targeted drug delivery approaches with liposomes by means of active targeting and passive targeting will help accomplish these goals of an ideal delivery system.

\subsubsection{Passive Targeting}

Passive targeting refers to the accumulation of the therapeutic agent or carrier at the target site due to physicochemical and physiological factors [107]. An understanding of the pathophysiology of the tumor is necessary to design a successful targeted delivery system. The ability to target drugs passively to the tumors using liposomes originated from the findings that the tumor blood vessels are more permeable to macromolecules than normal blood vessels [108, 109]. Tumor vessels have wide interendothelial junctions, large number of fenestrae and transendothelial channels formed by vesicles, and discontinuous or absent basement membrane [110]. Due to this leaky nature of the vasculature in the tumors, macromolecules can extravasate and accumulate in the tumors. In addition, the recovery of these macromolecules from tumors via blood or lymphatic drainage is impaired which results in high accumulation in the interstitial fluid compartment of the tumors $[25,111,112]$. This phenomenon of selective accumulation of macromolecules in the tumor is referred as enhanced permeability and retention (EPR) effect [113].

Passive accumulation of liposomes in the tumors occurs by transvascular transport across the blood vessels. The transport is determined by size of the liposomes, and diameter of the pores in the vasculature. The cutoff size for the vasculature in several tumors has been studied $[114,115]$. The cutoff size varies between tumors depending upon the stage of the tumor, size and location of the tumor. Tumors grown subcutaneously exhibited pore cutoff sizes between 200 to $1200 \mathrm{~nm}$. The cutoff was reduced in intracranial tumors and tumors undergoing regression. Nevertheless, the majority of tumors exhibited a vascular pore cutoff size between 380 and $780 \mathrm{~nm}$. So, the permeability of macromolecules will be independent of the pore size as long as the size 
of the molecule is less than the pore diameter. Liposomes used in cancer chemotherapy are typically between 100 and $300 \mathrm{~nm}$ in size which means that they can extravasate and selectively accumulate in most of the tumors.

The passive accumulation of liposomes in the tumors by EPR is a progressive phenomenon which requires several passages of the liposomes through the tumor blood vessels [51]. The liposomes should remain in circulation for a longer time to take advantage of selective accumulation in the tumors by EPR. However, the liposomes are considered foreign agents and are cleared by the reticuloendothelial system (RES) comprised of Kupffer cells of the liver and fixed macrophages of the spleen. Surface modifications of the liposomes with hydrophilic polymers such as PEG provides shielding effect and prevent the binding of opsonins to the liposomes which in turn minimize the recognition of these liposomes by RES [89]. If the liposomes remain in the circulation for a prolonged period of time, the chances of their accumulation by EPR in the tumor can be improved. Another important consideration for a longer circulation of liposomes is their size. The clearance of liposomes by RES increases with size [79]. The binding of opsonins and complement activation are dependent on size of the liposomes [81]. Even with the inclusion of PEG on the surface of the liposomes to minimize the clearance of the liposomes by RES, the cutoff size for the liposomes for long circulation remain between 150 and $200 \mathrm{~nm}$ [28].

\subsubsection{Active Targeting}

Passive accumulation of liposomes at the tumors does not necessarily elicit an improved therapeutic response which is dependent on the cellular uptake of the liposomes. Cellular uptake of the liposomes can be improved by active targeting approach. Active targeting refers to the attachment of specific ligands to the therapeutic agent or carrier system to recognize and interact with the target receptors or proteins at the cellular or tissue components [20,107, 116]. Although similar biodistribution and tumor accumulation is observed with liposomes by passive targeting and active targeting, an enhanced therapeutic response is accomplished with active targeting of liposomes directed against specific receptors due to improved cellular uptake $[117,118]$.

The primary role of targeting ligands is to enhance the cellular uptake into cancer cells and minimize the accumulation in normal tissue [22]. Thus, colloidal properties of liposomes will determine their biodistribution, and targeting ligands will ensure cellular uptake in the tumor. The number of ligands attached to the liposomes, distribution of target receptors in the tumors, and selectivity of the targeting ligands to those receptors will determine the intracellular uptake in the tumors. Under ideal conditions, the number of ligands bound to the tumors equals the number of available receptors [21]. The binding of targeting ligand is likely to have high nanomolar to low micromolar dissociation constants. So, more ligands are necessary than that may be accommodated to drive the equilibrium towards binding and receptor saturation. Once the receptors of the ligand are saturated, the specificity of the targeting ligand declines [119]. Excess of the targeted liposomes will be handled by the body as any non targeted liposomes. 
Tumor cells or endothelial cells of tumor vasculature over express a number of receptors which makes cancer a disease that will be largely benefitted by targeted drug delivery. In addition, unique expression of certain receptors by these cells can improve the specificity of targeting. To specifically target these receptors, ligands can be attached to the liposomes which on intravenous administration recognize these receptors. Binding of liposomes through these ligands to the target receptors results in an improved cellular uptake by receptor mediated endocytosis. The overall therapeutic effectiveness of the encapsulated drug can be improved while minimizing the exposure to normal cells.

\subsection{Targeting Receptors Expressed by Tumor Cells}

Several proteins are over expressed or uniquely expressed by tumor cells. Targeting ligands attached to the surface of liposomes should improve the cellular uptake by receptor mediated endocytosis. However, the accumulation of liposomes in the tumor microenvironment depends on passive targeting due to leakiness of the vasculature, longer circulation time, and nature of the liposomes. While passive targeting results in accumulation of the liposomes in tumor, receptor mediated endocytosis improves the cellular internalization.

\subsection{Antibodies as targeting ligands to target tumor cells}

Several antibodies can be used as targeting ligands for liposomes to specifically target proteins or receptors expressed on cancer cell surfaces. The antibodies can be covalently coupled with the reactive phospholipids in the membrane [120] or simply by hydrophobic bonding wherein the hydrophobic Fc portion of the antibody penetrates the lipid bilayer and immunologically active Fab portions extend into the aqueous phase and become available for interaction with receptors on the cell surface [121, 122]. The liposomes with antibodies attached to the surface of the liposomes are cleared rapidly by the RES. Coating of the liposome surface with hydrophilic polymers can help overcome the problems with clearance and improve circulation time. PEG coating of such liposomes with long PEG chains not only prevent the liposomes being recognized by the opsonins from plasma, but also hamper the interaction of the surface attached ligand with the target receptors. An interesting strategy was reported to overcome this issue by attaching the targeting ligand to the distal end of the PEG chains $[123,124]$. The binding of these immunoliposomes containing entrapped doxorubicin resulted in an increased cytotoxicity to murine squamous lung carcinoma cells as compared to liposomes without antibody [124].

The surface modification of liposomes with antibodies results in an enhanced uptake by the RES. Targeting ability with antibody depends on density of the antibody on the surface of the liposomes. Use of only Fab' fragment instead of whole antibody would minimize the RES uptake by avoiding $\mathrm{Fc}$ receptor mediated clearance mechanism. To improve targeting ability without compromising circulation time, Fab' fragments of monoclonal antibody $21 \mathrm{~B} 2$ specific for human carcinoembryonic antigen was coupled to 
the distal end of the PEG lipid with maleimide functional group [125]. These liposomes showed longer circulation time and lower RES uptake compared to the immunoliposomes prepared with the whole antibody. These liposomes also showed a two-fold higher accumulation in the tumor tissue, as demonstrated in a xenograft model in BALB/c nu/nu mice bearing MKN-45 human gastric cancer cell lines. Similarly, Fab' fragment was used in the preparation of Anti- human epidermal growth factor receptor 2 (HER2) immunoliposomes for targeting breast cancer [24]. Phage antibody selection strategy has been used to identify antibody fragments to optimize cellular internalization. Small chain $\mathrm{Fv}(\mathrm{ScFv})$ fragments of anti-HER2 antibody, ScFv F5 was found to specifically recognize the extracellular domain of HER2 and showed more internalization in a panel of HER2 over expressing cells [126]. Doxorubicin-loaded immunoliposomes containing Fab' or $\mathrm{ScFv}$ fragments have shown significant cure rates in HER2 over expressing mouse xenograft models [127].

Conjugation of antibodies to preformed liposomes containing coupling lipids, incorporated during liposomes formation, can leave reactive functional groups on the inner leaflet of bilayer and can potentially interfere with the encapsulated material [128, 129]. In addition, some of the coupling agents may be incompatible with the processing conditions of liposomes. Uster et al demonstrated that 1,2-distearoyl-sn-glycero-3phosphoethanolamine-N-(methoxy(polyethylene glycol)-1900) (MPEG $\left.{ }_{1900}-\mathrm{DSPE}\right)$ from micellar phase can be transferred in a time, and temperature dependant manner into preformed liposomes [130]. Post insertion of the micelles into preformed liposomes for the insertion of targeting ligands on to the external leaflet of liposomes was first demonstrated for oligopeptide and oligosaccharide ligands [129]. Ishida and coworkers designed doxorubicin-loaded immunoliposomes by post insertion method using sheep IgG or anti-CD19 monoclonal antibody coupled to micelles containing 1,2-distearoyl-snglycero-3-phosphoethanolamine-N-(methoxy(polyethylene glycol)-2000) ( $\mathrm{MPEG}_{2000^{-}}$ DSPE) and the coupling lipid malemide- $\mathrm{PEG}_{2000}$-DSPE [131]. A very little (4\%) doxorubicin was released in the transfer process, and anti-CD19 conjugated liposomes showed a three-fold increase in the association with human B lymphoma cell lines suggesting that the integrity and binding affinity of the these liposomes was not altered during the transfer process. The authors envisioned a combinatorial approach for making many different formulations of targeted liposomes with a simple procedure of selecting a vial of ligand-PEG-micelles for one-step incubation with the appropriate liposomal drug formulation.

\subsection{Folic acid}

Folic acid is a vitamin required for one-carbon transfer reaction in several metabolic pathways. Since folic acid is essential for the biosynthesis of nucleotide bases, its uptake is elevated in proliferating cells [132]. Of the two types of folate receptors, the folate receptor responsible for the internalization of oxidized folate by receptor mediated endocytosis is over expressed in cancer cells to compete for the vitamin available in limited quantity $[133,134]$. In normal cells, folate receptors are selectively expressed on the apical membrane surface. On malignant transformation, the polarity is reversed and 
the receptors are available for folate binding and internalization which makes them suitable for selective targeting.

The length of the spacer used in coupling procedure has been shown to play a crucial role in the ability of folic acid to interact with KB cells expressing folate receptors [135]. The liposomes with longer PEG spacer chains showed better internalization in the cells compared to the ones with smaller or no spacer chains. Pretreatment of the cells with excess free folate or antiserum against folate receptors competitively inhibited the uptake of liposomes indicating that the targeting was mediated through receptor mediated endocytosis. The use of folic acid conjugated PEG coated liposomes in targeted drug delivery was demonstrated in another study using doxorubicin-loaded liposomes [136]. The uptake of doxorubicin from these liposomes by KB cells was 45 and 1.6 times greater than non targeted liposomes or free drug respectively. The formulation also showed 86-fold higher cytotoxicity than non targeted liposomes and 2.7-fold higher cytotoxicity than free doxorubicin. The specificity of folate receptor mediated endocytosis was demonstrated in co-cultures containing human cervical carcinoma cell line Hela and human lung cancer cell line W138. Folate receptor over expressing Hela cells were killed completely following treatment with folate targeted liposomal doxorubicin leaving only folate non-expressing W138 cells in the culture dish. These results suggest that anticancer drugs can be targeted safely to cancer cells by folate mediated targeting while sparing normal cells.

Goren et al showed that doxorubicin from folate targeted liposomes was rapidly internalized, released in the cytoplasm, and detected in the nucleus within 1-2 hours [137]. This mechanism was suggested as a strategy to overcome multi drug resistance of drugs which are substrates for efflux transporters. The uptake of doxorubicin by multidrug-resistant subline of M109-HiFR lung cancer cells (M109R-HiFR) was not affected by P-glycoprotein (P-gp) mediated efflux which was in sharp contrast to the uptake of free doxorubicin based on verapamil-blockade experiments with quantitation of cell-associated drug, and flow cytometry analysis. The cells pretreated with folate targeted liposomes also inhibited the tumor growth as demonstrated by in vivo adaptive tumor growth assay in mice.

\subsection{Transferrin}

Iron $(\mathrm{Fe})$ is required in the active sites of the enzyme ribonucleotide reductase during DNA synthesis [138]. Due to redox activity and its potential to generate reactive oxygen species (ROS), the amount of free Fe is kept at minimum levels [139]. To control the amount of unbound $\mathrm{Fe}$, the metal is transported bound specifically to the two high affinity binding sites of serum glycoprotein, transferrin (Tf). The uptake of Fe by the cells involves binding of the Fe-loaded Tf to transferrin receptor1 (TfR1) present on the cell surface. Binding induces receptor mediated endocytosis of Fe-Tf [140]. TfR1 is a glycoprotein expressed in all nucleated cells at low levels [141]. The expression of TfR1 is elevated in proliferating cells which makes these receptors excellent choice for targeted 
delivery to cancer [142]. Active targeting to tumor can be accomplished via Tf or antibodies to TfR1.

The density of TfR 1 on the surface of cells is correlated with the extent of cell growth and division, and consequently, the tumor cells express more TfR 1 than normal cells. Eavarone et al demonstrated that transferrin coupled liposomes were taken up by C6 glioma cells through receptor mediated endocytosis [143]. These liposomes with Tf coupled to the distal ends of PEG spacers have shown an increased uptake of doxorubicin as compared to other non targeted liposomal formulations. Anabousi and coworkers demonstrated cytotoxicity of Tf-coupled liposomal doxorubicin on lung cancer cells [144]. In addition to TfR 1 mediated endocytosis, $\mathrm{pH}$ dependant cytoplasmic release was used as a strategy to improve the delivery of encapsulated materials in T-leukemia cells [145]. Kakudo et al showed that intracellular trafficking of the liposomes after receptor mediated endocytosis can be controlled by attaching a $\mathrm{pH}$ dependant fusogenic peptide on to the surface of the liposomes [146]. When fusogenic peptide, GALA was introduced into liposomal membranes using a cholesteryl moiety for anchoring (Chol-GALA), the encapsulated rhodamines were efficiently released and diffused into the cytosol.

Tf-PEG liposomes exhibited prolonged in vivo circulation time and low RES uptake in Colon 26 tumor bearing mice [147]. Longer circulation times resulted in enhanced extravasation of these liposomes in the tumor tissue. Electron microscopic analysis of the tumor tissues revealed that colloidal gold encapsulated Tf-PEG liposomes were internalized by receptor mediated endocytosis. The liposomes of size 60 and $120 \mathrm{~nm}$ showed higher accumulation in tumor tissue compared to 200 and $400 \mathrm{~nm}$ liposomes. Li et al showed that the tumor volume of mice bearing human hepatoma HepG2 tumor was reduced significantly by doxorubicin-loaded TfR1 targeted liposomes compared to non targeted liposomes and free drug [148]. Biodistribution studies indicated that doxorubicin can be efficiently targeted to tumors using liposomes while minimizing the uptake in heart and kidney. Cisplatin was delivered successfully by Tf targeted liposomes to mice bearing MKN45P gastric cancer with peritoneal dissemination [149]. Tf-PEG liposomes showed significantly higher survival rate compared to PEG liposomes, bare liposomes, and free drug. The mice in Tf-PEG liposome group maintained high liposome and cisplatin levels in ascites and showed a prolonged residence time in the peripheral circulation. Similarly, oxaliplatin-loaded Tf-PEG liposomes showed a reduced tumor growth ratio in mice bearing Colon 26 tumor [150]. Maruyama et al demonstrated improved survival rate in mice bearing Colon 26 tumor by boron neutron capture therapy (BNCT) with Tf-PEG liposomes encapsulated with mercaptoundecahydrododecaborate ${ }^{10} \mathrm{~B}(\mathrm{BSH})$ [151]. The tumor to plasma ratio of ${ }^{10} \mathrm{~B}$ was 6 after 72 hours of administration. Neutron irradiation after 72 hours of dosing showed improved tumor suppression compared to PEG liposomes, bare liposomes, and free BSH. Decahydrodecaborate- ${ }^{10} \mathrm{~B}$ (GB-10) have shown better therapeutic activity compared to BSH in BNCT using TfPEG liposomes in the treatment of squamous cell carcinoma in mice [152].

Specific binding, internalization, and intracellular delivery of liposomes conjugated with anti-Tf receptor antibody was evaluated in three different cell lines for acute $\mathrm{T}$ cell leukemia [153]. Anti-TFR liposomes showed effective binding and internalization in all 
three (MT-1, HUT-102, and Molt-4) cell lines. The growth inhibition of methotrexate- $\gamma$ aspartate in MT-1, and HUT-102 cells which express interleukin-2 (IL-2) receptors was 40 to 60 fold higher using anti-TFR liposomes compared to anti-Tac liposomes containing antibody directed against IL-2 receptors.

Transferrin receptor mediated endocytosis has been shown to circumvent P-gp mediated multi drug resistance. The uptake of doxorubicin was 3.5 fold higher in multi drug resistant SBC-3/ADM human lung cancer cells from Tf targeted liposomes as compared to free doxorubicin [154]. The liposomes made of egg phosphatidylcholine (EPC) showed better accumulation than the liposomes made of hydrogenated egg phosphatidylcholine (HEPC) suggesting that the fluidity of the liposomal membrane contributes to rapid internalization and drug release which in turn minimizes P-gp mediated efflux. Krieger et al showed that TfR1 targeted and also non targeted PEGylated cisplatin-loaded liposomes caused increased drug levels in cisplatin resistant A2780 ovarian carcinoma cells and resulted in higher cytotoxicities than the free drug [155]. Verapamil, a calcium channel antagonist with P-gp inhibitory activity was coencapsulated with doxorubicin in Tf-PEG liposomes to improve drug delivery to doxorubicin resistant K562 leukemia cells [156]. Cytotoxicity of Tf-PEG coencapsulated liposomes was greater than non targeted liposomes, and Tf-PEG doxorubicin-loaded liposomes.

A Tf liposomal formulation was evaluated for the delivery of 5-florouracil to brain [157]. The in vivo study showed selective uptake of Tf-liposomes from the brain capillary endothelial cells. The targeted liposomes showed 17, and 10 fold greater accumulation than free drug, and non targeted liposomes respectively [158]. Tf conjugated liposomes was proposed as strategy to improve the uptake of drug in brain. Paclitaxel was delivered to mice bearing C6 glioma tumors in the brain using Tf-PEG liposomes [159]. The uptake of Tf-PEG liposomes in the brain was supported by non invasive near infrared fluorescence (NIRF) imaging. Paclitaxel-loaded Tf-PEG liposomes showed a significant reduction in tumor burden compared to no treatment control. However, no significant reduction was observed with non targeted liposomes.

A major drawback with targeting tumor cells is that the drugs do not generally penetrate farther than three to five cell diameters from the blood vessels [160]. This leaves many cells distant from blood vessels with no drug or low levels of drug which increases the propensity to development of resistance.

\subsection{Targeting Receptors Expressed by Tumor Vasculature}

Endothelial cells within angiogenic blood vessels of solid tumors express several proteins which are either absent or expressed at very low levels in well developed normal blood vessels [161-163]. These receptors include integrins, aminopeptidases, and vascular endothelial growth factor (VEGF). Specific ligands can be attached to the liposomes to target these receptors for drug delivery to solid tumors. An attractive advantage of targeting tumor vasculature is that the tumor blood vessels are readily 
available for targeting than the tumor cells and thus specific targeting can be easily achieved.

\subsection{Integrins}

Peptides with Arg-Gly-Asp (RGD) or Asn-Gly-Arg (NGR) in a cyclic framework have been shown to bind specifically to tumor vasculature and cells over expressing $\alpha_{\mathrm{v}} \beta_{3}$ integrin receptors [163, 164]. Janssen et al demonstrated that RGD-PEG liposomes can be targeted specifically to proliferating endothelial cells using a Lewis lung carcinoma model in mice [165]. Intravital microscopy of skin fold chamber showed rhodamine labeled liposomes as fluorescent clusters in the tumor blood vessels within few minutes of administration. But PEG liposomes, and liposomes conjugated with control peptide were seen outside the tumor blood vessels indicating that these non specific liposomes extravasated deeper in to tumor interstitium. In another study, doxorubicin-loaded RGDPEG liposomes showed improved tumor regression in doxorubicin resistant Colon 26 tumors in mice [166]. The enhanced therapeutic efficacy in doxorubicin insensitive model suggested that the effect is mediated through binding of RGD-PEG liposomes to proliferating endothelial cells and not the tumor cells. Holig et al demonstrated that the concentration of RGD lipopeptide used in the liposomes played a crucial role in the blood circulation time [167]. The liposomes prepared with $0.1 \%$ of lipopeptide have shown prolonged circulation time compared to those with $1 \%$ lipopeptide.

Xiong and coworkers used flow cytometry and confocal microscopy to demonstrate integrin receptor mediated intracellular delivery of doxorubicin from RGD-PEG liposomes in B16 or A375 melanoma cell lines [168]. The uptake of RGD-PEG liposomes was greater than PEGylated liposomes but was less than free drug. Although the accumulation of RGD-PEG liposomes was greater in spleen compared to PEGylated liposomes, a better regression in tumor growth was observed in mouse melanoma model with the administration of single, and multiple doses. Replacing RGD peptide with RGD mimetic also provided better tumor regression compared to PEGylated liposomes and free drug $[169,170]$. Zhao et al demonstrated improved uptake and cytotoxicity of paclitaxel-loaded RGD-PEG liposomes in SKOV-3 human ovarian carcinoma cell lines [171]. The targeted formulation also showed significant inhibition of tumor growth in mouse ovarian cancer xenograft model. In another study, combretastatin A4 and doxorubicin were coencapsulated in RGD-PEG liposomes to improve the therapeutic efficacy in mouse melanoma model [105].

Patillo et al used $\alpha_{\mathrm{v}} \beta_{3}$ up-regulation in irradiated tumors to selectively deliver combretastatin-loaded RGD-PEG liposomes to melanoma in mice [172]. Treatment with RGD-PEG liposomes and tumor irradiation resulted in a significant delay in tumor growth compared to treatment without radiation.

Dubey et al showed that spontaneous lung metastasis and angiogenesis were prevented by RGD peptide anchored liposomes [173]. Cyclic RGD peptide anchored sterically stabilized liposomes containing 5-fluorouracil (5-FU) showed better activity 
against B16F10 melanoma primary tumor and metastasis than the non-targeted sterically stabilized liposomes and free drug. Murphy and coworkers evaluated the effect of doxorubicin-loaded RGD conjugated liposomes on primary tumor growth and spontaneous metastasis in orthotopic pancreatic and renal cell carcinoma models [174]. A reproducible modest effect on primary tumor growth and a substantial effect on metastasis were observed. Antitumor effect was observed with targeted liposomes at a dose one-fifteenth of the dose of free drug required to produce a similar effect.

\subsection{Aminopeptidase N (CD13)}

Endothelial cells of the angiogenic vessels over express membrane spanning molecules such as aminopeptidase N (CD13). NGR peptide has been shown to bind to aminopeptidase $\mathrm{N}$ isoform specific to the neovasculature, and can be used for targeting liposomes to angiogenic blood vessels. Pastorino et al used NGR conjugated doxorubicin-loaded liposomes for treating neuroblastoma in mouse orthotropic xenograft models [175]. The targeted liposomal formulation showed a complete regression of tumors in four out of six mice, and the two others showed more than $80 \%$ reduction in tumor mass. The blood vessel density was also suppressed to more than $90 \%$. These findings demonstrated that aminopeptidase N-targeted delivery of doxorubicin to blood vessels caused tumor regression because of its ability to promote apoptosis of the angiogenic endothelium. In addition, the mice treated with NGR liposomes did not show visible tumor metastasis. Garde et al demonstrated specific binding of NGR peptide conjugated liposomes in endothelial and tumor cells [176]. Doxorubicin was shown to internalize through the endosomal pathway, and then localize in cell nuclei. These liposomes exhibited selective cytotoxicity in CD13 expressing endothelial cells sparing CD13 negative colon cancer cells. Antitumor activity evaluation in PC3 prostate cancer xenograft showed significant inhibition of tumor growth and apparent reduction in vasculature. Antitumor effect was also improved in doxorubicin resistant HCT-116 colon carcinoma xenografts compared to free drug.

In addition to targeting aminopeptidase $\mathrm{N}$ of the tumor vasculature, targeting aminopeptidase A, over expressed on the tumor perivascular cells (pericytes), has been explored to complement tumor targeting. Sequential administration of aminopeptidase A, and then aminopeptidase $\mathrm{N}$ targeted liposome formulations resulted in an enhanced antitumor activity of doxorubicin compared to either targeting used alone in a neuroblastoma model [177].

\subsection{Vascular endothelial growth factor (VEGF)}

VEGF receptor is involved in the proliferation of vascular endothelial cells. Blocking the interaction between VEGF and VEGF receptors is a promising strategy to inhibit angiogenesis. Janssen and coworkers demonstrated that the peptide with the sequence ATWLPPR shows affinity for VEGF receptor. A ten-fold higher binding was observed with ATWLPPR conjugated liposomes in human vascular endothelial cell lines 
(HUVEC) compared to PEGylated liposomes. Kuesters and Campbell used bevacizumab, a humanized anti-VEGF-A monoclonal antibody to evaluate its targeting ability in human pancreatic cancer cell lines using cationic liposomes [178]. Bevacizumab conjugated liposomes showed an improved cellular uptake and tumor targeting of cationic liposomes compared to unmodified liposomes.

\subsection{Combination of Targeting of Tumor Vasculature, and Tumor Cells}

The combination of tumor vasculature targeting with tumor cell targeting can potentially overcome the drawbacks of both targeting approaches and improve the efficacy of targeted therapy. Pastorino et al demonstrated this concept by sequential administration of doxorubicin-loaded liposomes conjugated with anti-GD2 monoclonal antibodies directed to disialoganglioside receptor (GD2), widely expressed on cancer cells of neuronal origin. Then, NGR peptide conjugated liposomes directed to aminopeptidase $\mathrm{N}$ receptors, over expressed in tumor vasculature, was administered [179]. Evaluation of in vivo activity against two highly aggressive pseudo metastatic neuroblastoma animal models showed that there was a significant improvement in antitumor efficacy with the combination approach compared to either tumor cell or tumor vasculature targeting. It was suggested that tumor cell targeting results in direct killing of the cells, including cytotoxicity against cells at the periphery of the tumor and are independent of the tumor vasculature. On the other hand, vasculature targeted liposomes binds to and kills angiogenic blood vessels and consequently, kills the tumor cells supported by these cells, mainly in the tumor core.

\subsection{Dual Ligands to Improve Targeting}

Tumor cells over express some receptors which can be explored for targeting liposomes to tumors. It is rare that the target receptors are expressed exclusively by the tumor cells. These receptors are also expressed by normal cells but may not be at the same level as tumor cells which results in off-target accumulation of the targeted liposomes and toxicities associated with such binding. Fortunately, tumor cells over express multiple types of receptors. This property was explored by Saul et al for targeted drug delivery of liposomes with a dual targeting approach [180]. Doxorubicin-loaded liposomes with two targeting ligands, for folic acid receptors and epidermal growth factor receptors (EGFR), was developed and evaluated for cytotoxicity in KB cell lines. The liposomes with dual targeting showed significantly higher cytotoxicity in cells expressing both receptors compared to controls in which expression of one or both of the receptors were blocked. The results indicated that dual targeting can improve selectivity to target tumor cells while sparing off-target cells. Similarly, dual-targeting daunorubicin liposomes were developed by conjugating with $p$-aminophenyl- $\alpha$-D-mannopyranoside (MAN) and Tf for transporting drug across the blood brain barrier (BBB) and then targeting brain glioma [181]. The median survival time of tumor bearing rats was significantly longer with dual targeting compared to free daunorubicin, and other controls. Chen et al showed that multi-target RGD/octreotide-PEG-liposomes enhanced 
antitumor action of dihydrotanshinone as compared to single-target RGD-PEG liposomes and octreotide-PEG-liposomes in AGS cell gastric cancer xenograft model [182].

\subsubsection{Controlling Drug Release/Uptake from Liposomes}

Intracellular delivery of the drug is one of the key problems encountered in targeted drug delivery [183]. Targeted drug delivery of liposomes through receptor mediated endocytosis results in their entrapment in endosomes and eventually lysosomes. Active degradation process takes place in the lysosomes with the help of several lysosomal enzymes. By modification of the composition of liposomes, it is possible to control delivery of the drug. Some of the approaches used in improving intracellular delivery of liposomes in cancer chemotherapy will be discussed in the following sub sections.

\subsubsection{Cell-Penetrating Peptides}

Certain proteins and peptides can translocate across the plasma membrane and deliver their payload. This property can be used to overcome the barriers of intracellular delivery of liposomes to tumor cells. These proteins or peptides contain basic residues with domains of less than 20 amino acids, and are known as cell-penetrating peptides. Torchilin et al evaluated the effect of trans-activating transcriptional activator (TAT) protein on intracellular delivery of fluorescently labeled liposomes to Lewis lung carcinoma, BT20 human breast cancer, and H9C2 cardio myocyte cells [184]. Liposomes attached to TAT peptide using a PEG spacer showed high intracellular fluorescence. Liposomes with TAT attached directly to the liposome surface or to the PEG spacer shorter than the PEG chains used for steric stabilization did not show intracellular fluorescence indicating that free interaction between TAT and cellular surface is necessary for effective internalization. Low temperature or metabolic inhibitors, sodium azide or iodoacetamide did not show any effect on the translocation of TAT liposomes into cells confirming the energy-independent nature of this process. Tseng et al showed that the uptake of doxorubicin from TAT conjugated liposomes was increased by 12-fold in A431 human epidermoid carcinoma cells [185]. However, there was no difference in cytotoxicity or in vivo antitumor efficacy between liposomes with and without TAT. A $\mathrm{pH}$ responsive phospholipid was included in the formulation by Sawant and coworkers to improve TAT mediated intracellular uptake [186]. At low $\mathrm{pH}$ as in tumor

microenvironment, the hydrazone link of the PEG-Hydrazone-PE hydrolyzed resulting in loss of protective PEG which in turn and improved the internalization of liposomes due to better interaction of TAT with the cell surface. Marty et al demonstrated that functionalized Antennapedia (Antp, aa 43-58) conjugated liposomes loaded with cytotoxic drug N4-octadecyl-1-beta-D-arabinofuranosylcytosine-(5'- 5')-3'-Cethinylcytidine reduced IC50 by $70 \%$ on B16F1 melanoma cells compared to unmodified liposomes [187]. 


\subsubsection{2. $\quad \mathrm{pH}-$ Sensitive Liposomes}

The $\mathrm{pH}$-sensitive liposomes undergo selective destabilization on exposure to acidic environment. The rationale for the development of $\mathrm{pH}$-sensitive liposomes for drug delivery to tumors is to take advantage of the acidic microenvironment of the tumor to trigger the release of encapsulated contents. The $\mathrm{pH}$ of the interstitium rarely reaches a value below 6.5 and is difficult to tailor liposomes to be stable at physiological $\mathrm{pH}$ and to destabilize at this small window. But, the $\mathrm{pH}$ in the endosomes and lysosomes reach as low as 5.0 which makes it possible to develop $\mathrm{pH}$-sensitive liposomes that can destabilize following endocytosis. Several mechanisms of drug release from $\mathrm{pH}$-sensitive liposomes have been described [188, 189]. These liposomes can undergo $\mathrm{pH}$ induced fusion with endosomal membrane and release encapsulated contents in cytosol. They can undergo destabilization in the endosome and cause destabilization of the endosomal membrane resulting in release of the drug into the cytosol. They can also destabilize completely in the endosome, and the drug diffuse out of the endosomal membrane to reach cytosol, and other organelles.

The first class $\mathrm{pH}$-sensitive liposomes are composed of polymorphic phospholipids with acidic stabilizers to maintain integrity at neutral $\mathrm{pH}$. These liposomes are of less practical importance due to rapid clearance from the circulation. Kim et al used cholesterylhemisuccinate (CHEMS) as a pH-sensitive lipid in the development of gemcitabine-loaded epidermal growth factor receptor (EGFR) targeted liposomes [190]. In vivo evaluation in A549 alveolar carcinoma bearing mice showed that the rate of tumor inhibition improved with the $\mathrm{pH}$-sensitive targeted liposomes compared to other treatments. An increased apoptotic index revealed that the tumors treated with $\mathrm{pH}$ sensitive targeted liposomes resulted in an increased apoptosis of tumor cells, and consequently, increased tumor growth inhibition.

The second class of liposomes contains bonds cleavable at low $\mathrm{pH}$ to expose the destabilizing components of the liposomes resulting in release of encapsulated drug. Ishida et al developed doxorubicin dioleylphosphatidylethanolamine (DOPE) liposomes containing cleavable lipid derivative of PEG in which PEG was linked to distearoylphosphatidylethanolamine (DSPE) via disulfide linkage [191]. Exposure of these liposomes to $\mathrm{pH} 5.5$ resulted in release of encapsulated drug due to cleavage of the PEG chains and concomitant destabilization of the DOPE liposomes. These $\mathrm{pH}$-sensitive liposomes, targeted to the CD19 epitope on B-lymphoma cells, showed enhanced delivery of doxorubicin into the nuclei of the target cells and increased cytotoxicity compared to non-pH-sensitive liposomes. Survival studies in mouse B-cell lymphoma model showed that the $\mathrm{pH}$-sensitive liposomes improved the mean survival time of the animals compared to other controls.

Another class of $\mathrm{pH}$-sensitive liposomes uses $\mathrm{pH}$-sensitive peptides for destabilization. The fusogenic peptides undergo changes in confirmation with changes in

$\mathrm{pH}$ resulting in liposomes destabilization. They change from random coil at neutral $\mathrm{pH}$ to amphipathic helices at $\mathrm{pH} 5.0$ [192]. Self association of these structures following conformational changes results in their insertion into lipid bilayer, destabilization of 
liposomes, and release of encapsulated contents. Turk et al used a 29-amino acid peptide to enhance the cytotoxicity of cytosine arabinoside encapsulated in folate-targeted liposomes, and demonstrated a 30-fold increase drug potency in cultured KB cells [193].

The recent class of $\mathrm{pH}$-sensitive liposomes uses $\mathrm{pH}$-titratable polymers to destabilize membranes following change of the polymer conformation at low $\mathrm{pH}$. Upon acidification of the external medium, these polymers change from coil to globule phase, introduce curvature in the bilayer plane, induce membrane defects, and trigger release of entrapped cargo. The use N-isopropylacrylamide (NIPAM) as a pH-sensitive polymer was evaluated using cytosine arabinoside-loaded liposomes coupled with anti CD33 monoclonal antibody to target leukemic cells [194]. Flow cytometry and confocal microscopy analyses showed that the $\mathrm{pH}$-sensitive liposomes were efficiently internalized by various CD33+ leukemic cell lines. Highest cytotoxicity was observed with HL-60 cells treated with $\mathrm{pH}$-sensitive targeted liposomes compared to other treatments.

\subsubsection{Thermosensitive Liposomes}

Accumulation of liposomes in the tumor area does not guarantee that the drug is available inside the to the tumor cell to elicit therapeutic effect [195]. Use of mild hyperthermia, which involves heating of the tumor to temperatures up to $43^{\circ} \mathrm{C}$, along with thermosensitive liposomes can improve the drug delivery and therapeutic outcome. Hyperthermia works in two ways. It increases vascular permeability, improves the accumulation of liposomes in the tumor, and also sensitizes the cells for chemotherapy. Secondly, heat triggers can be used to release drugs from thermosensitive liposomes in close proximity to the cancer cells, and thereby, improve the uptake of drug by the cells. Yatvin et al first demonstrated that liposomes can be designed to release entrapped drug preferentially at temperatures attainable by mild local hyperthermia [196]. They showed that liposomes containing phospholipids that undergoes gel-liquid crystalline phase transition temperatures around $42^{\circ} \mathrm{C}$ can release encapsulated neomycin in the presence of serum with a release ratio of $100: 1$ at $44^{\circ} \mathrm{C}$ and $37^{\circ} \mathrm{C}$. Gaber and coworkers used in vivo fluorescence video microscopy to demonstrate the extravasation of liposomes, and the release of doxorubicin from thermosensitive liposomes in rat skin flap window chamber containing vascularized breast cancer [197]. The calculated intensity of free doxorubicin in the interstitial space was negligible at $34^{\circ} \mathrm{C}$, and increased by with heat to 38 -fold at $42^{\circ} \mathrm{C}$, and 76 -fold at $45^{\circ} \mathrm{C}$. Conventional thermosensitive liposomes required heating to temperatures of $43-45^{\circ} \mathrm{C}$. These temperatures are higher than clinically relevant and attainable temperatures which are in the range of $39-42^{\circ} \mathrm{C}$ [198]. Needham et al included a lysolipid to further improve the permeability at phase transition temperatures of the phospholipids [199]. The lysolipid 1-palmitoyl-2-hydroxy-snglycero-3-phosphocholine (MPPC) is kinetically trapped in the solid phase. At $\mathrm{T}_{\mathrm{m}}$, it melts and leaves the bilayer and enhances the permeability of the liposomes. These thermosensitive liposomes containing doxorubicin with mild hyperthermia showed highest tumor concentration of doxorubicin, and significant tumor regression in mouse

xenografts of human squamous cell carcinoma compared to conventional thermosensitive liposomes, non thermosensitive liposomes and free drug [200]. These liposomes are 
under clinical development under brand name TheromDox ${ }^{\circledR}$ (Celsion Corporation, New York, NY) for liver cancer and breast cancer.

\subsubsection{Target-Sensitive Liposomes}

Target-sensitive immunoliposomes have the ability to bind to the target specifically and destabilize upon target cell binding and release the entrapped drug. Ho et al developed target-sensitive immunoliposomes to specifically target herpes simplex virus (HSV) using phosphatidylethanolamine (PE) liposomes [201, 202]. PE cannot form stable liposomes by itself. Acylated monoclonal anti-HSV glycoprotein D stabilizes the bilayer phase of PE to form stable liposomes. The authors demonstrated that specific binding of these liposomes to HSV antigen containing glycoprotein D results in destabilization of the liposomes followed by release of encapsulated contents. Multivalent binding of these target specific liposomes were 10-fold more sensitive than the standard double-antibody sandwich enzyme-linked immunosorbent assay (ELISA) for the determination of HSV. In another study, they demonstrated the potential use of targetsensitive immunoliposomes for the delivery of cytotoxic nucleoside analogs into the virus-infected cells to inhibit virus replication using cytosine $\beta$-D-arabinoside, and acyclovir-loaded liposomes [203]. These liposomes have antibodies conjugated to N-glutaryl-phosphatidylethanolamine (NGPE) [204]. NGPE is equally distributed in the bilayer that stabilizes hexagonal phase forming lipids. Binding of these liposomes to the target cells result in capping of NGPE conjugated antibody, unequal distribution within the bilayer, and rapid destabilization. Hexagonal phase is formed releasing entrapped contents. Alternatively, liposomes with detachable polymer coating can be prepared [118]. These liposomes allow the flexibility of improving circulation time, and attaching targeting ligands to the distal end of PEG on the surface of the liposomes.

\subsubsection{Echogenic Liposomes}

Liposomes with air or other gases entrapped in the bilayer make them ultrasound active. These liposomes can be visualized at the target site, and can be disintegrated to release the encapsulated contents and by altering ultrasound energy. Alkan-Onyuksel et al developed inherently echogenic liposomes by lipid film hydration-sonication-freeze drying method [205]. The liposomes when lyophilized in the presence of mannitol formed echogenic liposomes. Mannitol has been shown to play a crucial role in improving echogenicity of liposomes. It crystallizes on freezing and subsequently causes defects in the liposomal bilayers [206]. These defects in bilayers remain in the cake and resealing takes place at the time of reconstitution entrapping air present in the atmosphere. Due to the presence of air, the liposomes have the potential for stress on activation by ultrasound and eventually release the contents at the target site. This phenomenon was explained by incorporating a fluorescent dye, calcein, into air-filled liposomes [35]. In vitro release studies showed an increase in the release of calcein with increasing applications of ultrasound. The release of air measured by a custom designed method was in agreement with the release of calcein. Kopechek et al compared the 
release of hydrophilic and lipophilic agents using color Doppler ultrasound in an exposure flow model [207]. Color Doppler effectively released hydrophilic calcein but not lipophilic papaverine. The authors explained this phenomenon by the fact that hydrophilic agents are entrapped in the aqueous core of the liposomes, the disruption of which by ultrasound results in the release of encapsulated contents into extraliposomal aqueous phase. On the other hand, lipophilic agents are strongly associated with the phospholipids in the bilayer. When the liposomes disintegrate, these agents may remain associated with phospholipids, may get incorporated in the micelles, but are not released into external aqueous phase.

The disintegration of microbubbles under ultrasound can also improve vascular permeability as demonstrated by extravasation of fluorescein isothiocyante (FITC) labeled dextran in chick embryo model [36]. Electron microscopy revealed micron-sized focal endothelial gaps, disseminated belbs, vacuoles and filopodia extending across tens of microns. Echogenic liposomes retain this property of microbubbles in addition to their ability to incorporate hydrophilic and lipophilic drugs, which makes them potential carriers for targeted drug delivery to solid tumors. These echogenic liposomes developed for imaging applications are $\sim 800 \mathrm{~nm}$ in diameter, and may be cleared rapidly by the RES. So, careful consideration of size and surface modification is necessary in developing echogenic liposomes for targeted drug delivery to solid tumors. Although this approach will be applicable only to tissues accessible by ultrasound, the long-circulating and targeting capability of these liposomes should take care of the tumors which are not accessible to ultrasound or those metastasized to other organs or tissues.

\subsubsection{Lyophilization to Improve the Stability of Liposomes}

Phospholipids from the liposomes can undergo hydrolysis and/or oxidation and result in physical degradation when stored as aqueous dispersions. As a result, the encapsulated drug tends to leak from the vesicles. Apart from leakage, the liposomes can aggregate or fuse together resulting in an increase in particle size distribution. In a stability stand point, loss of encapsulated drug or increase in vesicle size is unacceptable. Freeze drying or lyophilization can prevent hydrolysis of phospholipids and physical degradation during storage of the liposomes [34]. However, lyophilization process itself can cause loss of encapsulated drug and fusion of the vesicles. Interaction of water with hydrophilic phosphate head groups of phospholipids plays a key role in the formation of liposomes and hence, removal of water presents more challenges. The ability of several carbohydrates to protect liposomes during lyophilization is described by two hypotheses. First is 'water replacement' hypothesis in which sugar molecules interact with phospholipids and replace water molecules in the microenvironment during dehydration [208-210]. In such case sugars can maintain the hydrated environment in a dried state. The vesicles can also be stabilized in the glassy state of good glass formers such as trehalose and sucrose. These sugars can encase the membranes in their amorphous matrix and thereby, impart stability as described by 'vitrification' hypothesis [211-213]. 
Organisms such as brine shrimp cysts and some species of nematodes survive dehydration which is related to their ability to produce nonreducing disaccharide trehalose $[214,215]$. These anhydrobiotic organisms survive for several years in dry state and return to normal metabolic activities on rehydration. Using this analogy, the ability of trehalose and other sugars to maintain the structure, and integrity of liposomes was evaluated by Madden and coworkers [33]. The results showed that trehalose when used at concentrations of $125 \mathrm{mM}$ or higher protected the liposomes from structural changes of dehydration. The entrapped tracers ${ }^{22} \mathrm{Na}^{+}$, and $\left[{ }^{3} \mathrm{H}\right]$ inulin were retained in the vesicles in presence of trehalose. The protection effect was greater when trehalose was present on both sides of the membrane as compared to when it was present only on the outer part of the membrane. To further evaluate the ability of trehalose to prevent leakage of drugs, adriamycin encapsulated liposomes were dehydrated in presence of $250 \mathrm{mM}$ of trehalose. More than $90 \%$ of the drug was retained following drying and rehydration. The protection effect of trehalose was attributed to its ability to replace water molecules normally hydrogen-bonded to the polar head groups of lipids.

Crowe et al evaluated the interaction of various carbohydrates on phospholipid monomolecular layers formed between air-water interface [216]. Trehalose and sucrose interacted with dipalmitoylphosphatidylcholine (DPPC) monolayer producing an increased area per molecule, and increased membrane fluidity than normally observed with water. This effect showed a strong correlation with the ability of trehalose and sucrose to stabilize membrane structures. They provided a more detailed explanation for the protection by trehalose using sodium isocitrate-loaded liposomes [217]. Freeze fracture study of fresh, dry, and rehydrated liposomes revealed that the liposomes freeze dried in the presence of trehalose were of the same size before and after drying and after rehydration. But the liposomes freeze dried without trehalose fused to form multilamellar liposomes or unilamellar liposomes of size about four-fold greater than fresh liposomes. The study on effect of trehalose on gel to liquid crystalline transition temperature using differential scanning calorimetry (DSC) showed that trehalose reduced the transition temperature such that the dried phospholipid remain in liquid crystalline phase at room temperature. The rehydrated form of liposomes also contains lipids at liquid crystalline phase, and hence there is no transition during rehydration. In contrast, the liposomes dried without trehalose remained at gel phase in room temperature. On rehydration, the lipids in these liposomes have undergone a transition to liquid crystalline phase. The transition of gel phase to liquid crystalline phase during rehydration was postulated as a mechanism for leakage of liposomal encapsulated contents. Results obtained with infrared spectroscopy showed that trehalose and DPPC interact by hydrogen bonding between the -OH groups of trehalose and polar head groups of DPPC [208]. It was shown that hydrogen bonding alters the spacing of the polar head groups and thereby decreases van der Waals interactions in the hydrocarbon chains of the DPPC.

Residual water content was measured to compare the effect of different carbohydrates on their ability to interact with 1-palmitoyl-2-oleoyl-sn-glycero-3-phosphocholine (POPC) [218]. As the trehalose content was increased there was no significant change in residual water content but it increased sharply when critical trehalose concentration was reached, producing two regions. In region I, all of the trehalose interacts with 
phospholipid and is unavailable for binding with water. In region II, free trehalose is available for binding with water at a similar rate seen with trehalose lyophilized without lipid. The results suggested that the ability of different sugars to protect the liposomal bilayers depends on their strength of direct interaction with phospholipids and not on the residual water content. Maltose and trehalose showed high interaction with the lipid followed by sucrose and glucose. Inositol showed little or no interaction with the lipid.

Fluorescence resonance energy transfer assay was used to demonstrate the protection effect of lyoprotectants against fusion of liposomes by incorporating donor and acceptor fluorophores into two separate liposome populations and then freeze drying with or without trehalose. The results indicated that the fusion was inhibited by almost $90 \%$ when trehalose is present on both inner and outer part of the membrane [219]. Maltose, sucrose, glucose, and fructose also prevented fusion of liposomes to a lesser extent than trehalose, but, this effect was evident only at high concentrations of these carbohydrates. Comparatively, trehalose prevented fusion at much lower concentrations than other carbohydrates. In contrast, raffinose, cellobiose, inositol, and glycerol showed minimal inhibitory effect on fusion. The relative abilities of these carbohydrates to inhibit fusion correlated with their ability to interact with phospholipids, maintain fluidity, and preserve biological membranes.

The ability of different saccharides to act as cryoprotectants for small unilamellar vesicles was evaluated with high resolution ${ }^{1} \mathrm{H}-\mathrm{NMR}$ (nuclear magnetic resonance), Electron spin resonance (ESR) spin labeling and DSC [220, 221]. The study with sucrose, trehalose, and glucose showed that the stabilization effect is not sugar specific but is a general property of saccharides. The ability of these saccharides to replace water molecules of hydration by forming hydrogen bond with the polar phospholipid group, at same binding site as water, was suggested as the mechanism for cryoprotection. The fraction of liposomes unaffected during dehydration was dependant on the sugar to lipid molar ratio.

Molecular dynamics simulations was used to characterize interaction of trehalose, maltose, and glucose with DPPC bilayer at atomic resolution [222]. These sugars interacted directly with phospholipid head group by hydrogen bonding. At higher temperature, these sugars prevented thermal disruption, and the protective effect correlated with the number of sugar-phospholipid head group hydrogen bonds. The ability of disaccharides to provide better protection compared to monosaccharides was attributed to their ability to bridge three or more lipid molecules. Trehalose preserves the integrity of biological membranes under thermal stress. But, trehalose alone does not preserve the integrity of phospholipid bilayers under mechanical stress in the form of lateral stretching [223].

Fourier transform infra red (FTIR) spectrometric studies to determine hydrogen bonding of different phosphatidylcholines with cholesterol, and galactose revealed a downfield shift for $\mathrm{P}=\mathrm{O}$ asymmetric stretch when galactose was present alone or in combination with cholesterol [224]. These results were consistent with molecular dynamics simulations with trehalose [225], suggesting that there is a hydrogen bonding 
interaction between $\mathrm{P}=\mathrm{O}$ of the phospholipid and $-\mathrm{OH}$ group of the carbohydrates during lyophilization [218].

A study with increasing amounts of internal trehalose on retention of carboxyfluorescein-loaded liposomes showed that only a small amount of trehalose is required inside. Adding more sugar inside had no apparent effect on stability [226]. The authors also described that retention of the marker in the liposomes depends on the mass ratio of sugar and lipid and not the concentration of sugar in the bulk phase.

In contrast to previous reports on internal/external ratio of carbohydrates in the liposomes for lyophilization, Ohtake et al reported that a combination of high inner/outer trehalose concentrations showed a decrease in $\mathrm{T}_{\mathrm{m}}$ of DPPC or DPPC-cholesterol containing liposomes in the hydrated state [210]. The decrease in $\mathrm{T}_{\mathrm{m}}$ was further amplified in the dehydrated state. The extent of $\mathrm{T}_{\mathrm{m}}$ decrease is diminished with an increasing exterior concentration of trehalose.

During freezing process, water crystallizes to form ice crystals. In this process, the vesicles and remaining solute are highly concentrated. Inclusion of lyoprotectant can form an amorphous matrix around the vesicles and protect the vesicles from fusion and prevent rupture caused by the crystallization of ice. Koster et al showed that vitrification of sugars occurs in the intermembrane region, and the resulting glassy state mechanically hinders conformational changes of lipid bilayers [212, 227]. These changes further result in alteration of phase behavior of the membranes. In the absence of sugars, $T_{m}$ of the lipids increased during lyophilization. Inclusion of sugars diminished this increase of $\mathrm{T}_{\mathrm{m}}$. Thermal analysis by DSC showed that sugars vitrifying around liquid crystalline phase of lipids lowered $\mathrm{T}_{\mathrm{m}}$ below the transition temperature of the fully hydrated lipid, $\mathrm{T}_{\mathrm{o}}$. On the other hand, sugars vitrifying around gel phase of lipids increased $\mathrm{T}_{\mathrm{m}}$ during the first heating scan and, then lowered it below $\mathrm{T}_{\mathrm{o}}$ in subsequent heating scans of the sample. However, vitrification alone is not sufficient to depress the $T_{m}$ of lipids in dry state, but both vitrification, and direct interaction between phospholipid head group and carbohydrates is required [209].

The effect of freezing rate on the integrity of liposomes was evaluated using carboxyfluorescein-loaded liposomes in presence of sucrose, trehalose, and glucose as lyoprotectants [228]. The results showed that quick freezing in liquid nitrogen induced a stress vector on DPPC containing liposomes. The stress was realized only after drying and rehydration. This stress was dependant on composition of the liposomes i.e., inclusion of cholesterol in the formulation minimized the leakage. The leakage was further minimized by slower cooling rate of $0.5^{\circ} \mathrm{C}$ during freezing. In contrast, a change in freezing rate did not affect retention of carboxyfluorescein in EPC containing liposomes. The potential of modulated DSC (MDSC) to study the thermal properties of freeze dried and frozen phospholipid and carbohydrate mixtures has been described [229]. The MDSC measurements of slowly and quickly frozen liposomes showed that the glass transition for quick freezing occurred at lower temperatures than slow freezing. This effect with slow freezing was attributed to progression of freeze concentration to completion before the freeze concentrate solidifies. This leaves a smaller amount of water 
that remains in sugar resulting in a higher glass transition. Viscoelastic properties can also be used to determine the effect of freezing rates on storage stability [230]. Longer shear relaxation times were observed for cationic liposomal formulations prepared by slow freezing compared to those prepared by quick freezing. The formulation prepared by slow freezing have a lower matrix mobility which is an indication of better storage stability.

The lyoprotectants used in the formulation of liposomes should not only protect the liposomal bilayer against instability to prevent fusion and leakage of the encapsulated drug but also protect the linkers or molecules attached to the surface of the liposomes from degradation during lyophilization. Friede et al prepared liposomes with maleimide functional groups, and lyophilized in presence of sorbitol [231]. These liposomes served as shelf items for the preparation immunogenic liposomes or peptide conjugates. The results showed that the liposomes retained their maleimide functional groups on rehydration of lyophilized liposomes. The liposomes previously conjugated to a peptide also retained its immunogenic activity on lyophilization followed by rehydration.

Multivesicular liposomes containing cytarabine was prepared by water-oil-water double emulsification method and the stability was evaluated for aqueous dispersions [232]. Almost no drug leaked from the liposomes when the concentration of trehalose on the outer part of the membrane was isotonic. Leakage was observed at higher concentrations of trehalose and when the liposomes were suspended in deionized water. The protection effect was explained on the basis of osmotic gradient. When the liposomes are suspended in hypertonic solution, the vesicles are subjected to shrinkage whereas hypotonic conditions as with deionized water results in expansion and rupture of the vesicles. Both these conditions can result in leakage of the drug. So the outer concentration of trehalose was kept constant at $9 \%$, and the inner trehalose concentration was varied from 0 to $18 \%$ during lyophilization to study the effect of inner trehalose concentration of liposomes containing different phospholipids on leakage. Multivesicular liposomes made of EPC showed higher retention of cytarabine than DPPC liposomes which showed greater retention than DOPC liposomes after lyophilization. The retention increased with increasing inner trehalose concentrations up to $9 \%$ and then leveled off, which showed that only a small amount of trehalose is required in the inner part of the membrane and further addition of higher amounts of sugar does not have additional advantages.

\subsection{HYPOTHESIS AND SPECIFIC AIMS}

Breast cancer is the most common type of cancer and second leading cause of death from cancer in western women. About $20-30 \%$ of women diagnosed with breast cancer develop metastatic breast cancer. The most successful way of treating metastatic breast cancer is systemic therapy in the form of chemotherapy or immunotherapy. Paclitaxel is one of the most effective chemotherapeutic agents used in the treatment of metastatic breast cancers. It is an antimicrotubule agent that promotes the assembly of microtubules from tubulin dimers and stabilizes microtubules by preventing depolymerization. 
Systemic administration of paclitaxel is associated with serious toxic effects including myelosuppression and peripheral neuropathy, in addition to hypersensitivity reactions due to the formulation components. So, development of formulation to specifically deliver paclitaxel to the tumor is necessary.

Entrapment of paclitaxel in the bilayer of liposomes can alter pharmacokinetics and biodistribution of the drug, and thereby, minimize systemic toxicity and improve therapeutic efficacy. The liposomal composition needs to be optimized to maximize the loading of paclitaxel. Even though high loading can be obtained, retention of the loaded drug in the bilayer of liposomes is important to maintain storage stability. The optimal loading that can be retained in the liposomes during storage needs to be determined and factors affecting the same needs to be optimized.

Conventional liposomes are cleared rapidly by the RES. The in vivo circulation time can be improved by circumventing RES uptake by reducing the size of the liposomes and/or by attaching hydrophilic polymers such as PEG onto the surface of the liposomes. Liposomes of size 100-300 nm should also improve localization of paclitaxel in the tumor by passive targeting via EPR effect due to leaky nature of the tumor vasculature, and impaired lymphatic drainage.

Active targeting via receptor mediated endocytosis can be accomplished by modifying the surface of the liposomes with targeting ligands specific to the receptors expressed by the endothelial cells of tumor vasculature or tumor cells. Actively proliferating endothelial cells of tumor vasculature over expresses $\alpha_{\mathrm{v}} \beta_{3}$ integrin receptors. Peptides with RGD motif in a cyclic framework (cRGD) have been shown to effectively bind to $\alpha_{v} \beta_{3}$ integrin receptors. Surface modification of paclitaxel-loaded liposomes with cRGD peptide attached to the distal end of PEG chains should improve the localization of paclitaxel in the tumor. TFR1 receptors are over expressed by proliferating tumor cells. Transferrin attached to the surface of the liposomes by means of PEG spacer and a linker should improve the uptake of paclitaxel by the breast cancer cells through receptor mediated endocytosis.

Long term storage stability of liposomes is a major concern due to leakage of the encapsulated drug and fusion/aggregation on storage. Lyophilization of the liposomes can overcome these drawbacks and improve long-term stability. The conditions used during lyophilization itself can be damaging to the bilayer of liposomes which requires careful selection or optimization of the formulation and process.

In addition to the localization and uptake of these liposomes by natural cytosolic mechanisms, external triggers can be used to improve the permeability at the vasculature to improve localization. Design of paclitaxel-loaded targeted liposomes with air/gas incorporated in the bilayer will make these liposomes ultrasound active. Ultrasound can be used as an external trigger to disintegrate these liposomes to improve vascular permeability, and the uptake. 
The objective of this dissertation work is to develop targeted liposomal formulations to improve localization of paclitaxel in tumor sites, and therefore, minimize toxicity, and improve the efficacy of the treatment of breast cancers. To accomplish this objective the following hypotheses are envisaged.

I. Paclitaxel-loaded gas-filled liposomes can be developed so that they can be visualized with ultrasound, and disintegrated using high intensity ultrasound pulses

II. Development of stable paclitaxel-loaded liposomes conjugated to either transferrin or cRGD peptide should improve the efficacy of the treatment of breast cancer

To test these hypotheses, my specific aims were to:

1) Develop prototype formulation of cRGD conjugated gas-filled liposomes. In this specific aim feasibility of developing ultrasound active, RGD conjugated targeted liposomes was evaluated. Formulation and process parameters critical to the target profile were identified and optimized to achieve an acceptable prototype formulation that can be utilized as a platform for drug-loaded liposomes for targeted drug delivery.

2) Optimize paclitaxel loading in the liposomal formulation. Formulation and process parameters that influence paclitaxel loading in the liposomes were identified and optimized. Stability of the liposomal dispersion was evaluated for up to 10 days to determine the optimum composition of liposomes without the risk of leakage. Storage stability of the optimized liposomal dispersion was evaluated for three months.

3) Develop cRGD conjugated paclitaxel-loaded gas-filled liposomes. The formulation and process parameters were optimized to obtain paclitaxelloaded cRGD conjugated gas-filled long-circulating liposomes (Pac-RGDGFTLCL). The echogenicity, and the ability to disintegrate these liposomes was evaluated using in vitro models with ultrasound. Stability of the formulation was evaluated for three months.

4) Evaluate biodistribution and efficacy of the liposomes targeted to tumor vasculature, and to compare the efficacy of liposomes targeted to tumor vasculature and tumor cells in a mouse breast cancer model. Mouse xenografts bearing human MDA-MB-231 breast cancer were used for the evaluation of biodistribution and efficacy of the formulations. The localization cRGD conjugated liposomes were compared with long-circulating liposomes, and solution formulation using non invasive near infrared fluorescence (NIRF) imaging. The localization of paclitaxel-loaded liposomal formulations, and solution formulation was evaluated by measuring tumor tissue concentrations after 24 hours of administration. The efficacy of paclitaxelloaded long-circulating liposomes (Pac-LCL), and paclitaxel-loaded cRGD conjugated targeted long-circulating liposomes (Pac-RGD-TLCL) were compared to normal saline control, and paclitaxel solution formulation. To compare the effect of targeting tumor cells, paclitaxel-loaded transferrin 
conjugated targeted liposomes (Pac-Tf-TLCL) were administered to another group of animals. The effect of these formulations on tumor volume was monitored as a measure of efficacy.

5) Lyophilize the paclitaxel-loaded liposomes to improve long-term storage stability. The formulation was optimized to minimize the leakage of paclitaxel, and fusion/aggregation of the liposomes. Stability of the lyophilized formulation was evaluated for Pac-LCL, Pac-RGD-TLCL, and Pac-Tf-TLCL. 


\section{CHAPTER 2. DEVELOPMENT OF PROTOTYPE FORMULATION OF GAS- FILLED TARGETED LIPOSOMES}

Ultrasound is the transmission of pressure waves through a medium at frequencies above 20,000 Hz. Microbubbles are ultrasound contrast agents used in radiology for imaging organs and tissues accessible to ultrasound. Ultrasound travels more slowly in gas compared to aqueous medium. The difference in speed causes an acoustic impedance mismatch between tissue or blood surrounding the microbubbles [37]. As a result, microbubbles act as efficient reflectors of ultrasound energy, and used as contrast agents for imaging. The microbubbles can undergo cavitation, which can be explored for drug delivery applications. Cavitation is the formation and/or activity of microbubbles in a medium exposed to ultrasound [233]. When a medium containing microbubbles is exposed to ultrasound, the microbubbles expand at low pressures and contract at high pressures. If the resulting oscillation is stable over time with continuous expansion and contraction cycles, it is called 'stable' or 'non inertial' cavitation [38]. This oscillation causes micro streaming around the bubble with velocities and shear rates proportional to the amplitudes of oscillation. If the intensity of ultrasound is increased, the amplitude of oscillation increases such that the inward moving wall of the bubble obtain sufficient inertia that it cannot reverse the direction when the ultrasound pressure is reversed. The microbubble is compressed continuously creating high pressures within the bubble resulting in 'collapse' or 'inertial' cavitation. The collapse cavitation is detrimental to cells and vesicles because of high shear stress in the region of collapse, shock waves produced by the collapse, and free radicals generated due to high temperature. If the collapse occurs near a solid surface, an asymmetric collapse takes place producing liquid jets at sonic speeds which can pierce blood vessel walls. Stieger et al demonstrated that low frequency contrast enhanced ultrasound can increase vascular permeability resulting in convective extravasation of fluorescent labeled dextran in a chick embryo model [36].

Liposomes are biocompatible carriers with ability to incorporate both lipophilic and hydrophilic drugs [23]. Incorporation of gas in the bilayer of liposomes makes them acoustically active [205]. These echogenic liposomes have the potential for stress on activation by ultrasound, and eventually release the contents at the target site [35]. This phenomenon exhibited by echogenic liposomes can be explored for drug delivery applications. But, the size of these echogenic liposomes used for imaging is $\sim 800 \mathrm{~nm}$, and would be cleared rapidly by the RES. The freeze drying method used for the preparation of echogenic liposomes causes an increase in the size of liposomes. Crystallization of mannitol present in the formulation causes rupture in the bilayer during the freeze drying process $[35,234]$. In a dry cake, air passes through these gaps and fills up the bilayer. The ruptured bilayer reseals during reconstitution, entrapping air inside the bilayer. The extent of damage to the bilayer caused by crystallization of mannitol determines the amount of air/gas entrapped in the bilayer, which is reflected as final size of the liposomes. Since the use of liposomes of size greater than 150-200 nm is unsuitable for drug delivery due to rapid clearance by the RES [28], smaller sized liposomes are desired. By using suitable lyoprotectants and/or surfactants at adequate 
proportions, we can control the crystallization of mannitol and minimize the increase in size of liposomes.

An ideal carrier for therapeutic targeting to solid tumors must have a longer circulation time than those used for imaging and should possess targeting capability. In vivo circulation time can be increased by reducing the size of liposomes and/or modifying the surface with PEG derivatives [28]. Targeting capability can be achieved by attaching specific ligands onto the surface of the liposomes. The $\alpha_{v} \beta_{3}$ integrin receptors are highly expressed in actively proliferating endothelial cells of tumor vasculature and tumor cells [163, 235]. Peptides with Arg-Gly-Asp (RGD) motif in a cyclic framework have been shown to bind to these receptors and are effective in targeting solid tumors [172]. By attaching RGD peptide to the surface through PEG spacers, these liposomes can be targeted to solid tumors. The objective of this work is to identify and optimize the formulation and process parameters, and to develop a prototype parenteral formulation that serves as a platform for drug-loaded ultrasound active liposomes for targeted delivery to solid tumors.

\subsection{MATERIALS AND METHODS}

\subsubsection{Materials}

The phospholipids used in this work were all of research grade. Hydrogenated soy phosphatidylcholine (HSPC) and 1,2-distearoyl-sn-glycero-3-phoshoethanolamine-N(poly(ethylene glycol)2000) (DSPE-PEG 2000 ) were obtained from Northern Lipids (Burnbay, BC, Canada). 1,2-distearoyl-sn-glycero-3-phoshoethanolamine-N-(maleimide poly(ethylene glycol)2000) (maleimide DSPE-PEG 2000 ) was obtained from Avanti Polar Lipids (Alabaster, AL). Cholesterol and sucrose were purchased from Sigma (St. Louis, MO). Mannitol was obtained from Mallinckrodt Chemicals (Phillipsburg, NJ). Cyclo (Arg-Gly-Asp-D-Phe-Cys) peptide (cRGD) was obtained from Peptides International (Louisville, KY). Poloxamer 188 was obtained from BASF (Mount Olive, NJ). High performance liquid chromatography (HPLC) grade water, acetonitrile, and methanol were obtained from Fisher Scientific (Fair Lawn, NJ). HPLC grade chloroform was purchased from Acros Organics (Morris Plains, NJ). N-2-hydroxyethylpiperazine-N'-2ethanesulfonic acid (HEPES) buffer, 1M was obtained from Mediatech (Manassas, VA). All other chemicals and reagents were of analytical grade and used without further purification or characterization.

\subsubsection{Preparation of Long-Circulating Liposomes (LCL)}

Long-circulating liposomes were prepared by the lipid film hydration-extrusion method using HSPC, DSPE-PEG 2000 , and cholesterol. The lipid film was prepared by dissolving phospholipids and cholesterol in 9:1 chloroform-methanol. The solution thus obtained was evaporated in a round bottom flask using rotary evaporator under reduced 
pressure at $45^{\circ} \mathrm{C}$ for $4-6$ hours. The dry film was hydrated with $50 \mathrm{mM}$ HEPES buffer $\mathrm{pH}$ 6.5 at $65^{\circ} \mathrm{C}$ for $45-60$ minutes. The multilamellar vesicles (MLVs) thus obtained were sized by sonication using Misonix Sonicator 3000 probe sonicator (Misonix, Inc., Farmingdale, NY) in the initial experiments for the evaluation of composition of liposomes. For the rest of the experiments, the liposomes were sized by extrusion through stacked polycarbonate membrane filters of varying pore diameters under high pressure to obtain small unilamellar vesicles (SUVs). The SUVs were characterized for particle size distribution, zeta potential, and morphology. The pore diameter of filters, pressure, and number cycles used in the extrusion process were optimized to obtain an average particle size of $110 \pm 20 \mathrm{~nm}$.

\subsubsection{Verification of Conjugation Reaction between cRGD and Maleimide DSPE-PEG 2000}

The conjugation of RGD peptide was verified by mass spectrometry by the method reported by Desu [236] with slight modifications using Micromass Q-tof-2 ${ }^{\mathrm{TM}}$ mass spectrometer (Micromass, Manchester, UK). The cRGD peptide and maleimide DSPE$\mathrm{PEG}_{2000}$ were dissolved separately in $50 \mathrm{mM}$ HEPES buffer $\mathrm{pH} 6.5$, and mixed in equimolar ratio. The reaction mixture was incubated at $25^{\circ} \mathrm{C}$ for 12 hours, and the unconjugated peptide was removed by dialysis through 2000 molecular weight cut-off (MWCO) Slide-A-Lyzer dialysis cassette against $50 \mathrm{mM}$ HEPES buffer $\mathrm{pH} 6.5$ at $5 \pm$ $3^{\circ} \mathrm{C}$ for $6 \mathrm{~h}$. The conjugate was desalted through a Sephadex G-25 macro spin column (Harvard Apparatus, Holliston, MA) before analysis. For analysis, $20 \mu \mathrm{L}$ of the conjugate was diluted with $0.3 \%$ formic acid and injected to the electrospray ionizer at a flow rate of $0.5 \mu \mathrm{L} / \mathrm{min}$. The capillary and cone voltages were $2.80 \mathrm{KV}$ and $45 \mathrm{~V}$ respectively.

\subsubsection{Preparation of cRGD Conjugated Targeted Long-Circulating Liposomes (RGD-TLCL)}

For the preparation of RGD-TLCL, a part of DSPE-PEG 2000 was replaced with maleimide DSPE-PEG 2000 and the liposomes were prepared as described in section 2.1.2 to obtain SUVs with reactive maleimide functional group. The maleimide-LCL thus obtained was conjugated with cRGD peptide by incubation at $25^{\circ} \mathrm{C}$ for $12 \mathrm{~h}$. The unconjugated peptide remaining after the reaction was removed from the liposomes by equilibrium dialysis through 10,000 MWCO Slide-A-Lyzer dialysis cassettes (Thermo Fisher Scientific, Rockford, IL) against $50 \mathrm{mM}$ HEPES buffer $\mathrm{pH} 6.5$ at $5 \pm 3^{\circ} \mathrm{C}$ overnight. The conjugation efficiency was determined by quantifying free RGD in the dialyzate by reversed phase high performance liquid chromatography (RPHPLC).

\subsubsection{Determination of Conjugation Efficiency}

The conjugation efficiency was determined by equilibrium dialysis through 10,000 MWCO cellulose membrane using reusable dialyzers (Harvard Apparatus, Holliston, 
MA). The amount of unreacted RGD in the dialyzate was quantified by RPHPLC as a measure of the conjugation efficiency. The HPLC system comprised of Waters 600 Controller, Waters 717 Plus Autosampler, and Waters 2996 Photodiode Array Detector (Waters Corporation, Milford, MA) was used. The RPHPLC analysis was done using a Nova-Pak $\mathrm{C}_{18}$ column $(3.9 \times 150 \mathrm{~mm}, 4 \mu \mathrm{m})$ (Waters Corporation, Milford, MA). The gradient elution was performed using $0.05 \% \mathrm{v} / \mathrm{v}$ trifluoroacetic acid (TFA) in water (A) and $0.05 \% \mathrm{v} / \mathrm{v}$ TFA in acetonitrile (B) with a linear gradient of 10 to $60 \% \mathrm{~B}$ in $50 \mathrm{~min}$ at a flow rate of $1 \mathrm{~mL} / \mathrm{min}$ The retention time for RGD was between 13 and 14 min at a detection wavelength of $220 \mathrm{~nm}$.

\subsubsection{Determination of Particle Size and Zeta Potential}

The average diameter (Z-avg) of the liposomes was determined using dynamic light scattering using Zetasizer Nano ZS (Malvern Instruments, Westborough, MA) equipped with $4.5 \mathrm{~mW}$ diode laser as a source of light operating at $670 \mathrm{~nm}$. Particle scattered photons were detected at an angle of $173^{\circ}$. The samples were suitably diluted with HPLC grade water for the determination of Z-avg. Three independent measurements were performed for each sample. Zeta potential was determined from electrophoretic mobility using samples suitably diluted with HPLC grade water.

\subsubsection{Preparation of Gas-Filled LCL (GFLCL) and Gas-Filled RGD-TLCL (RGD-GFTLCL)}

The LCL or RGD-TLCL was mixed with equal volume of mannitol solution (0$17.5 \% \mathrm{w} / \mathrm{v})$ containing sucrose $(0-10 \% \mathrm{w} / \mathrm{v})$ as a lyoprotectant, and poloxamer $188(0-$ $0.5 \% \mathrm{w} / \mathrm{v}$ ) as a surfactant. For the initial experiments to evaluate the composition of liposomes, the liposome-mannitol mixture was frozen at $-80^{\circ} \mathrm{C}$, and then freeze dried using Labconco FreeZone Plus 6 bench top freeze drier (Labconco Corporation, Kansas City, MO). For rest of the experiments, the lyophilization cycle was designed based on the glass transition temperature of maximally freeze-concentrated amorphous phase $\left(\mathrm{Tg}^{\prime}\right)$, determined by differential scanning calorimetry (DSC). Lyophilization was carried out using a lab scale Virtis Genesis 25ES lyophilizer (The Virtis Company, Gardiner, NY). The primary drying temperature was selected based on $\mathrm{Tg}^{\prime}$ and the mixture was lyophilized in $5 \mathrm{cc}$ glass lyo vials. The moisture content of the dry cake was monitored to ensure end point of secondary drying. On completion of the drying cycle, the vials were sealed under a partial vacuum. Perfluoropropane was filled in the headspace above the vials at atmospheric pressure to obtain GFLCL and RGD-GFTLCL. The procedure used in the preparation of RGD-GFTLCL is summarized in Figure 2-1. The physical appearance of the dry cake was recorded as quality control parameter. The vesicle size, zeta potential, and echogenicity were monitored in reconstituted samples. The vials were stored at $5 \pm 3^{\circ} \mathrm{C}$ until further use. 

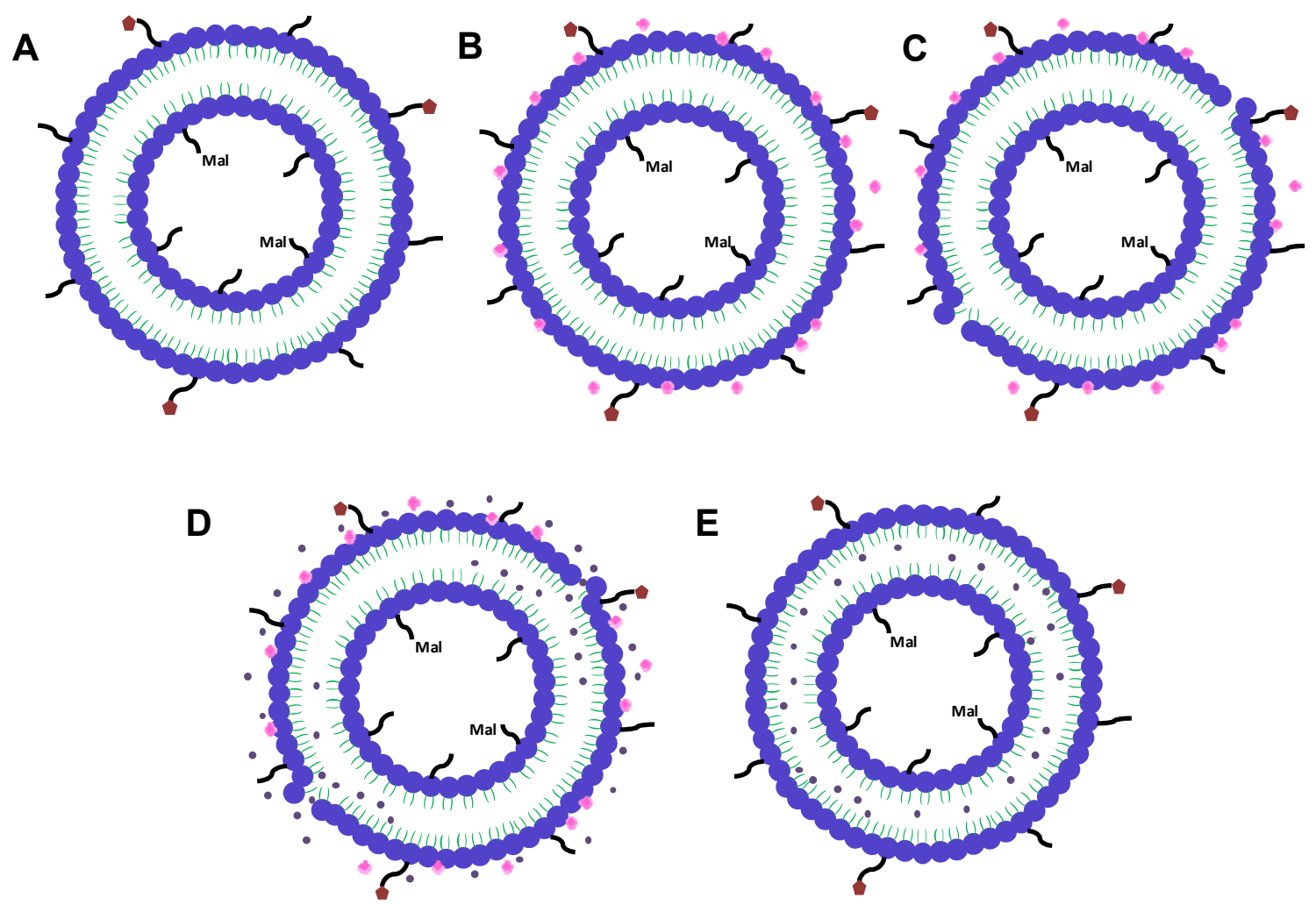

Figure 2-1. Cartoon representing the method of preparation of RGD-GFTLCL

Notes. The process of preparation of RGD-GFLCL is as follows: A. The liposomes are prepared by lipid film hydration extrusion method and then conjugated with cRGD peptide. B. The SUVs are mixed with mannitol ( $\diamond)$ solution. C. Lyophilization in presence of mannitol results in rupture of the bilayer due to crystallization of mannitol. D. The dried cake is equilibrated with perfluoropropane $(\bullet)$ filled in the head space of the vials. E. Reconstitution with water results in resealing of the bilayer with perfluoropropane entrapped in the bilayer. The diagram is not drawn to scale. 


\subsubsection{Thermal Analysis by DSC}

The effect of lyoprotectant and surfactant on crystallization of mannitol was monitored by DSC using a Q2000 differential scanning calorimeter equipped with refrigerated cooling system (TA Instruments, New Castle, DE). About 10-20 $\mu \mathrm{L}$ of the liposome mixture prior to lyophilization was placed in T-Zero aluminum pans and hermetically sealed. An empty pan prepared in a similar manner was used as reference. The samples were equilibrated at $25^{\circ} \mathrm{C}$ and then cooled at $5^{\circ} \mathrm{C} / \mathrm{min}$ to $-70^{\circ} \mathrm{C}$. After equilibration, the samples were heated at the rate of $10^{\circ} \mathrm{C} / \mathrm{min}$ to $25^{\circ} \mathrm{C}$. The effect of sucrose and poloxamer 188 on crystallization peak of mannitol was evaluated. $\mathrm{The}^{\mathrm{Tg}} \mathrm{g}^{\prime}$ was also determined from these thermograms to design the lyophilization cycle.

\subsubsection{Determination of Moisture Content}

The moisture content of the lyophilized cake was determined using an Orion AF8 Volumetric Karl Fischer Titrator (Orion Research Inc., Boston, MA). The titrator was calibrated on each day of analysis with a known quantity of water. About $75 \mathrm{mg}$ of the dried sample was weighed accurately and added to the titration vessel. The titration was started after allowing one minute for extraction.

\subsubsection{Determination of Echogenicity}

Acoustic activity of GFLCL and RGD-GFTLCL was characterized using an in vitro method utilizing Phillips ATL HDI-5000 ultrasound system and broadband L12-5 transducer (Philips Healthcare, Andover, MA). A schematic representation of the set up used in the measurement of echogenicity is provided in Figure 2-2. It consists of a rectangular plexiglass chamber filled with $15 \mathrm{~mL}$ of normal saline (NS). A small magnetic stir bar was placed in the chamber to enable uniform mixing. The chamber was then placed on a magnetic stirrer. The transducer was positioned such that it has direct contact with NS filled in the tank. The images were acquired with a mechanical index setting of 0.1 . The gas-filled liposomes were reconstituted with HPLC grade water. After

acquiring the background image for $5 \mathrm{sec}$., $200 \mu \mathrm{L}$ of the reconstituted liposomal product was injected into the tank with a $1 \mathrm{cc}$ syringe, and the images were acquired for one minute. All image sequences were analyzed offline using a custom program using MATLAB 7.0.4 software (The MathWorks, Inc., Natick, MA). The pixel values in the region of interest (ROI) were compared to the pixel values in the background. The number of folds increase in mean gray scale value (MGSV) in the ROI over the background was calculated as the measure of acoustic activity.

\subsubsection{Characterization of Morphology of Liposomes}

Morphology of the liposomes was characterized by transmission electron microscopy (TEM) using a negative staining technique. Sequential two droplet method was used for 


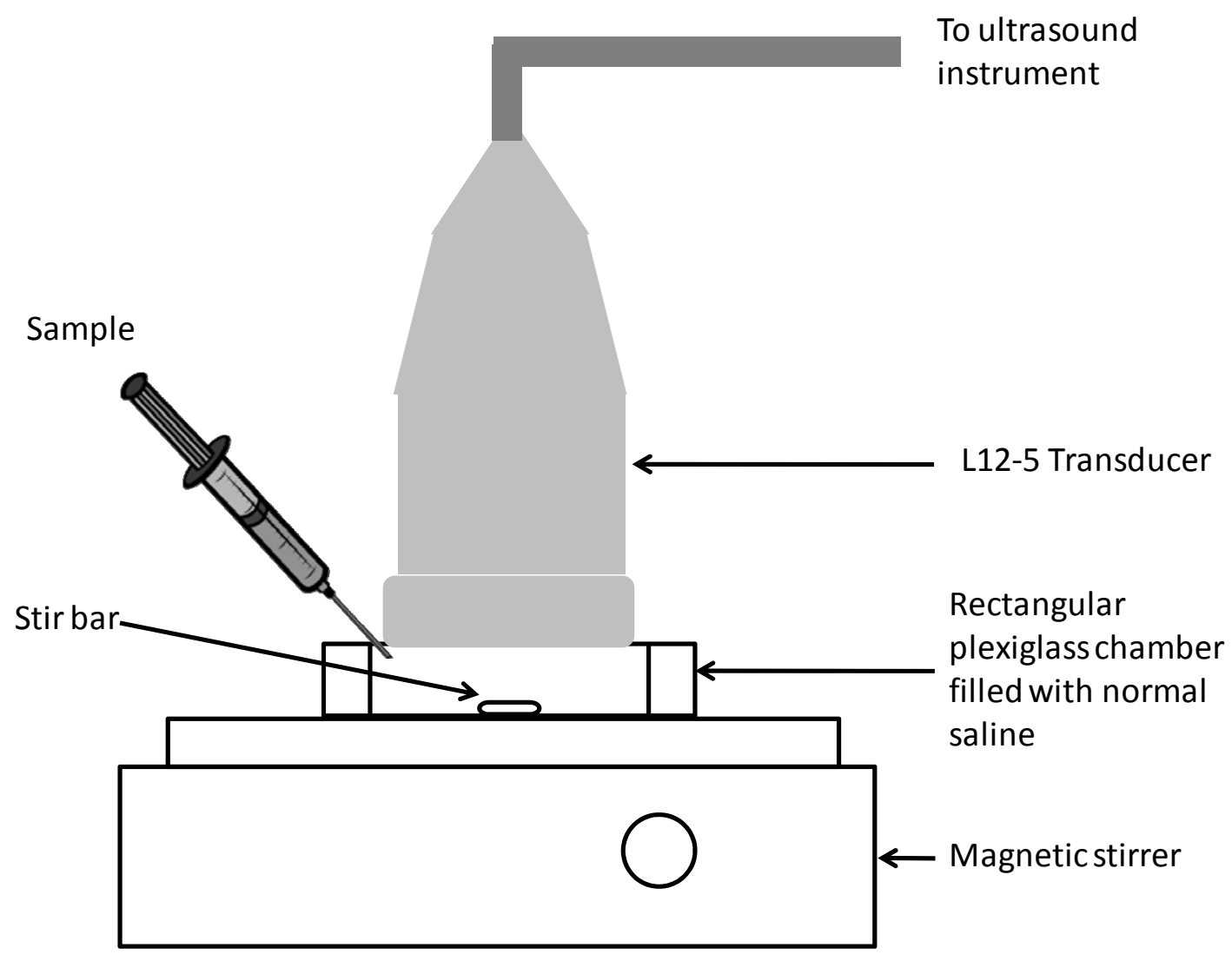

Figure 2-2. Schematic representation of the in vitro technique used for the measurement of echogenicity

Notes. The apparatus used for the determination of echogenicity consists of a rectangular plexiglass chamber filled with normal saline. A magnetic stir bar was placed in the chamber to enable uniform mixing. The ultrasound transducer was immersed in normal saline up to not more than $0.5 \mathrm{~cm}$ from the top of the plexiglass chamber. The reconstituted echogenic liposomes were injected in to the tank using a syringe. The diagram is not drawn to scale. 
staining liposome samples with 2\% w/v uranyl acetate on 400-mesh formvar support film on copper specimen grid (Electron Microscopy Sciences, Hartfield, PA). The TEM images were acquired using JEOL 2000EX transmission electron microscope (JEOL USA, Inc., Peabody, MA) equipped with high resolution digital camera.

\subsection{RESULTS AND DISCUSSION}

\subsubsection{Effect of Liposomal Bilayer Composition}

The effect of composition of the bilayer of liposomes on the particle size distribution (PSD) was evaluated before and after freeze drying. These liposomes were sized by sonication using probe sonicator, and the liposome-mannitol mixture was freeze dried using bench top freeze dryer. Figure 2-3 shows the effect of increasing the concentration of cholesterol on the PSD after freeze drying. An increase in size was observed with all the bilayer compositions. The trend shows that cholesterol minimizes the increase in size of liposomes after freeze drying. Cholesterol is known to reduce the fluidity and improve the rigidity of the liposomes [59]. The higher rigidity in turn minimizes the rupture of the bilayer caused by the crystallization of mannitol. Since the final size of the liposomes after freeze drying depends on the amount of gas entrapped in the liposomes which depends on the extent of rupture to the bilayer caused by mannitol, the increase in rigidity provides better control on the final size of GFLCL. The composition with HSPC: DSPEPEG2000: Cholesterol in a ratio 45:5:50 was selected for further studies. The sonication process for sizing resulted in a high batch to batch variability (data not shown) even though the conditions were optimized and strictly controlled. Hence, an extrusion process was used for further studies. Similarly, the bench top freeze dryer did not have the options to control product temperature. So, further studies were carried out using a more robust lab scale lyophilizer.

\subsubsection{Effect of Lyoprotectant and Surfactant}

Mannitol undergoes crystallization from the frozen aqueous solutions during the warming phase of freeze drying [237-239]. The crystallization of mannitol has been shown to play a critical role in the preparation of echogenic liposomes [206]. The crystallization causes rupture in the bilayer of liposomes due to which air/gas is entrapped in the liposomes. But these liposomes developed for ultrasound contrast imaging were $\sim 800 \mathrm{~nm}$ in diameter, and can be cleared rapidly by the RES. For drug delivery applications, liposomes of size $150-200 \mathrm{~nm}$ is desired to escape clearance by the RES [28]. Since the final size of these echogenic liposomes depends on the amount of gas entrapped in the liposomes which is determined by the extent of rupture to the bilayer caused by the crystallization of mannitol, we attempted to control the crystallization of mannitol to develop echogenic liposomes of size $<200 \mathrm{~nm}$. The crystallization of mannitol can be controlled by altering the process parameters during freeze drying and by additives [238, 240, 241]. In our preliminary experiments, we evaluated sucrose and 


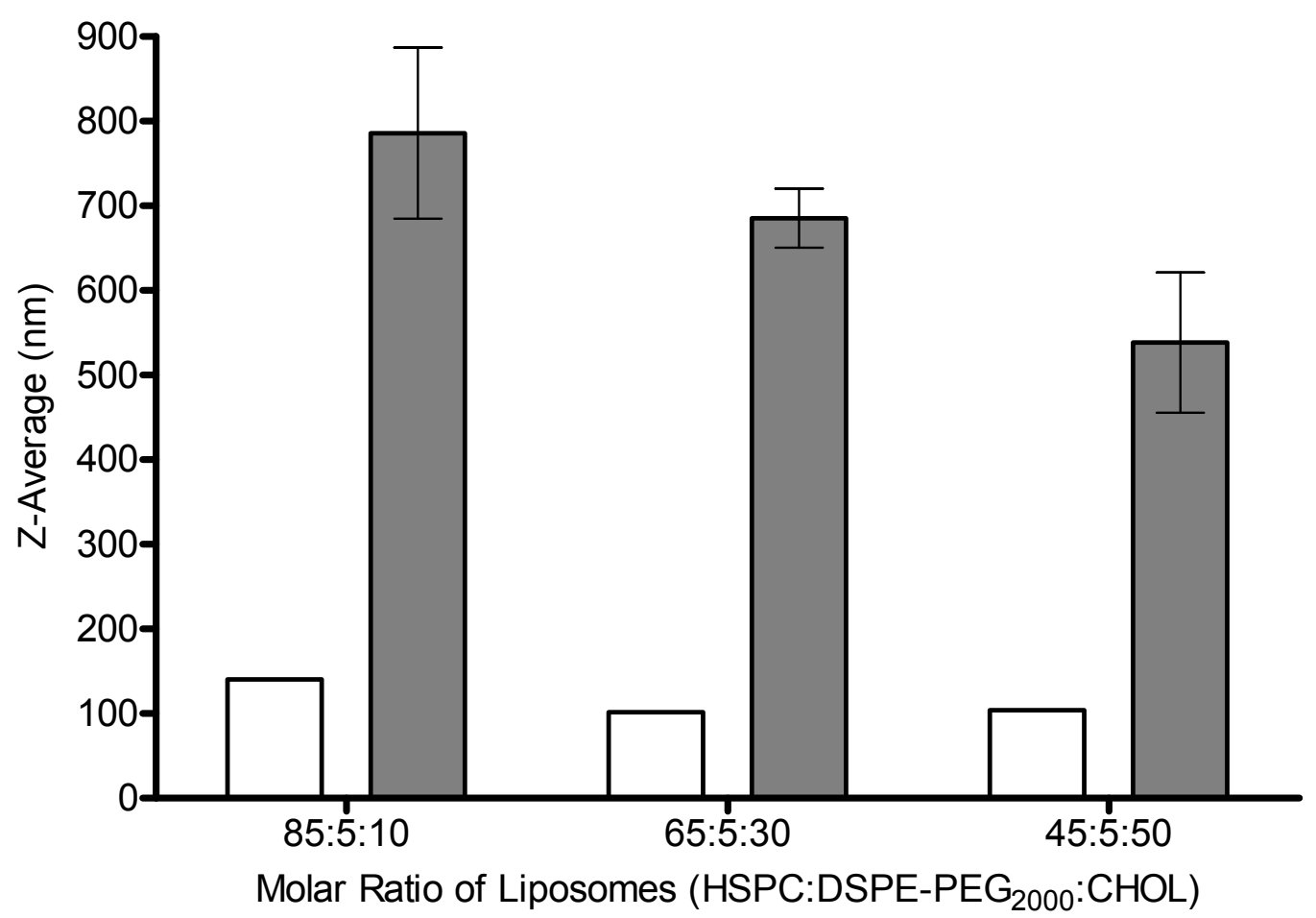

Figure 2-3. Effect of liposome composition on PSD of liposomes after freeze drying

Notes. The liposomes were prepared by lipid film hydration sonication method. The PSD was determined before and after freeze drying in presence of mannitol. The freeze dried gas-filled liposome samples were reconstituted with HPLC grade water for the measurement of PSD. The results indicate mean \pm standard deviation of three independent measurements ( $\square$ Before lyophilization; $\square$ After lyophilization). 
trehalose as lyoprotectants, and poloxamer 188 and Solutol HS15 as surfactants. The combination of sucrose and poloxamer 188 provided better control on PSD (Data not shown), and hence they were selected for further evaluation. Figure 2-4 shows DSC heating profile of frozen liposomes in presence of mannitol and sucrose as a lyoprotectant. When only mannitol was present, an exothermic transition characteristic of crystallization of mannitol was observed with an onset temperature of $-25.23^{\circ} \mathrm{C}$ and a peak temperature of $-18.52^{\circ} \mathrm{C}$. The crystallization peak was suppressed by sucrose showing that the crystallization of mannitol can be controlled by the inclusion non crystallizing solute sucrose in the formulation for lyophilization. In contrast, the surfactant poloxamer 188 did not show any effect on the crystallization of mannitol. The lyophilization cycle for the preparation of GFLCL was designed based on $\mathrm{Tg}^{\prime}$, determined by DSC. The Tg' of the formulations containing mannitol and sucrose was around $-42^{\circ} \mathrm{C}$ and hence the primary drying temperature was set at $-45^{\circ} \mathrm{C}$. The influence of sucrose was also evident on the PSD of liposomes after lyophilization. Figure 2-5 shows the effect of mannitol-sucrose ratio on the PSD before and after lyophilization. The liposomes prepared in presence of mannitol without lyoprotectant showed more than five-fold increase in size. This increase in size was minimized by the addition of lyoprotectant sucrose. Increasing concentrations of sucrose in the formulation reduced the final size of GFLCL. This effect could be attributed the control of crystallization of mannitol by the inclusion of non crystallizing solute sucrose in the formulation. Control in crystallization resulted in minimal rupture to the bilayer and thereby minimal entrapment of gas in the liposomes. The effect of surfactant on the PSD of liposomes after lyophilization is depicted in Figure 2-6. Although there was no evidence on control of crystallization of mannitol from DSC, the addition of surfactant, poloxamer 188 showed a decrease in the final size of GFLCL. This effect could be attributed to the formation of monolayer on the surface of liposomes which in turn minimize the aggregation due to steric effects.

\subsubsection{Effect of Sucrose Concentration on Echogenicity}

The effect of increasing concentrations of sucrose in the formulation on echogenicity of the GFLCL was evaluated in a custom in vitro model. The data is presented in Figure 2-7. A maximum increase in gray scale intensity was observed in the ROI with GFLCL formulation that was lyophilized without sucrose. Inclusion of sucrose reduced the echogenicity of GFLCL. The trend was similar to that observed with the PSD. A decrease in echogenicity was expected with the addition of sucrose because the smaller liposomes contain less amount of gas per vesicle compared to larger liposomes. The high echogenicity in the mannitol containing formulation serves as further proof of the role of crystallization of mannitol on size and echogenicity. In contrast, the formulation without mannitol also showed echogenicity similar to the formulation containing both mannitol and sucrose suggesting that other factors are also involved in determining echogenicity of liposomes. The mean grayscale intensity in the ROI for all the formulations decreased with time on continuous exposure to ultrasound. The decrease in intensity could be due to loss perfluoropropane from vesicles due to diffusion or due to disintegration induced by ultrasound. The formulation with intermediate mannitol-sucrose ratio of $4: 3$, and a 


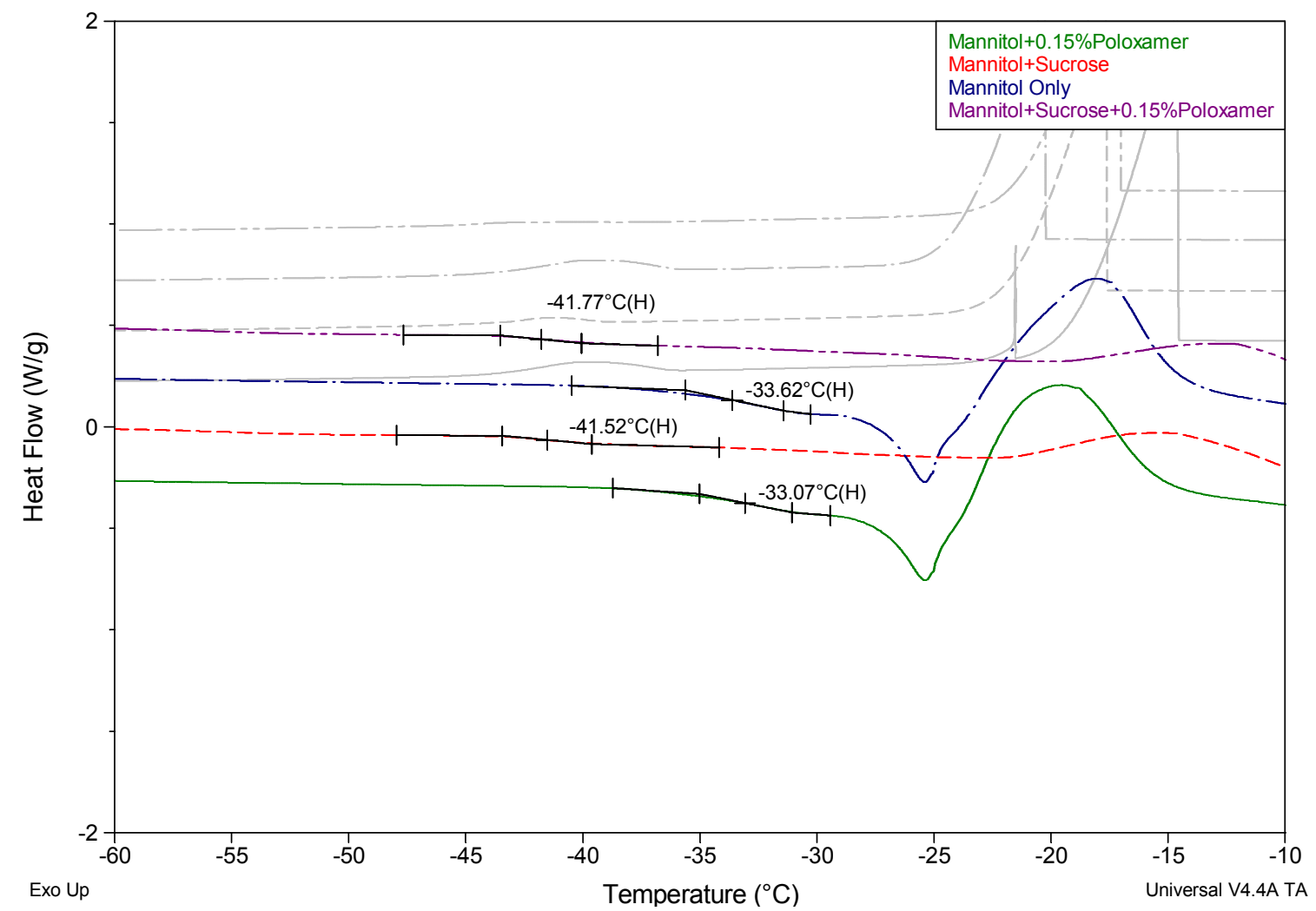

Figure 2-4. DSC thermograms showing the effect of sucrose and poloxamer on crystallization of mannitol

Notes. The liposomes were mixed with equal volume of mannitol solution with or without sucrose or poloxamer 188 . The samples were hermetically sealed in aluminum pans, and cooled at the rate of $5^{\circ} \mathrm{C} / \mathrm{min}$ to $-70^{\circ} \mathrm{C}$. After equilibration at $-70^{\circ} \mathrm{C}$, the samples were heated at the rate of $10^{\circ} \mathrm{C} / \mathrm{min}$ to $25^{\circ} \mathrm{C}$. The heating profile is shown in the figure. Heat flow is shown on an arbitrary scale. 


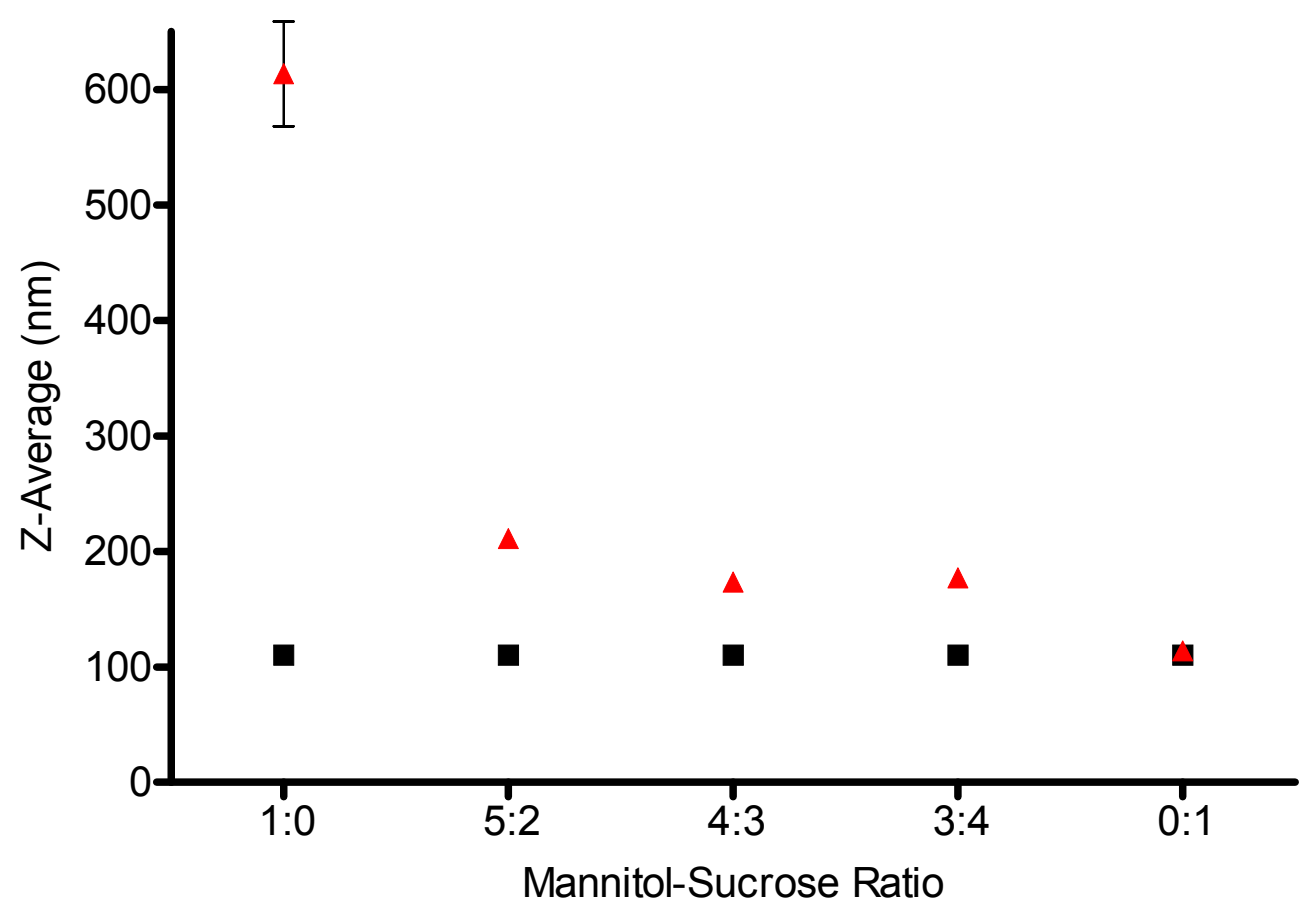

Figure 2-5. Effect of mannitol-sucrose ratio on PSD of liposomes after lyophilization

Notes. The liposomes were prepared by lipid film hydration extrusion method. The PSD was determined before and after freeze drying in presence of varying ratios of mannitol and sucrose in presence of $0.15 \% \mathrm{w} / \mathrm{v}$ poloxamer 188 . The freeze dried gasfilled liposome samples were reconstituted with HPLC grade water for the measurement of PSD. The results indicate mean \pm standard deviation of three independent measurements ( $\bullet$ Before lyophilization; $\Delta$ After lyophilization). 


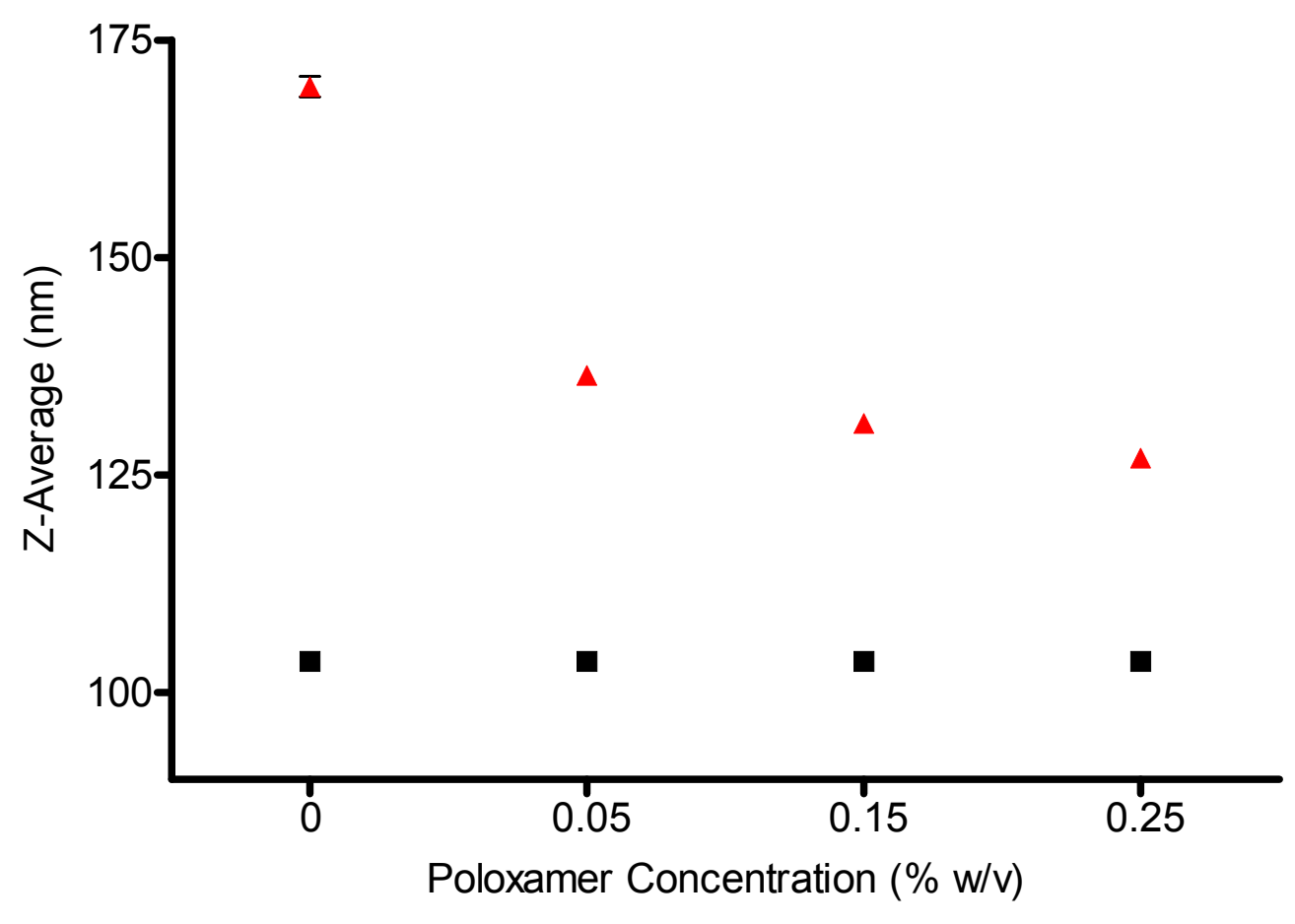

Figure 2-6. Effect of poloxamer on PSD of liposomes after lyophilization

Notes. The liposomes were prepared by lipid film hydration extrusion method. The PSD was determined before and after freeze drying in presence of mannitol and sucrose at varying concentrations of poloxamer 188. The freeze dried gas-filled liposome samples were reconstituted with HPLC grade water for the measurement of PSD. The results indicate mean \pm standard deviation of three independent measurements ( $\square$ Before lyophilization; $\Delta$ After lyophilization). 


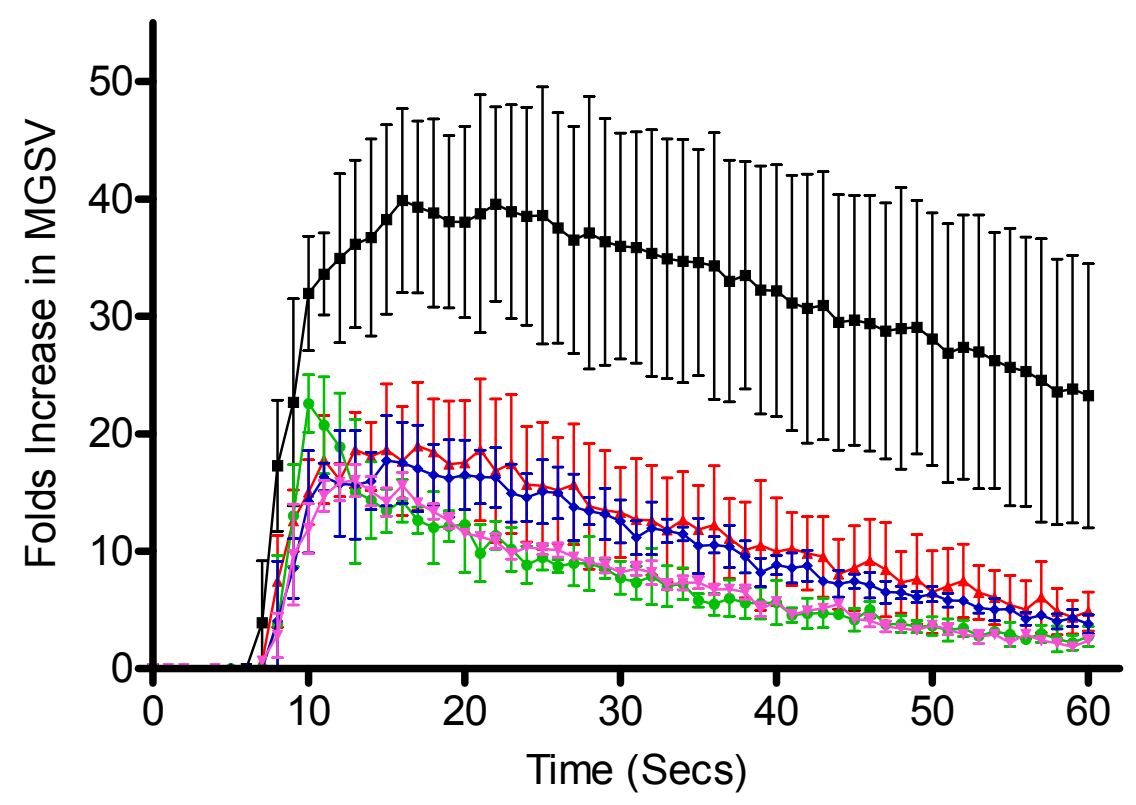

Figure 2-7. Effect of mannitol-sucrose ratio on echogenicity of GFLCL

Notes. The echogenicity was determined in an in vitro model with Philips ATL-HDI 5000 ultrasound system and L12-5 broadband transducer. The freeze dried gas-filled liposome samples were reconstituted with HPLC grade water for the measurement of echogenicity. After acquiring background images for 5 sec., $200 \mu \mathrm{L}$ of the sample was added using a syringe, and the acquisition was continued for up to one minute. The data was processed off-line with a custom MATLAB program. Three ROIs were randomly selected, and the average increase in mean grayscale intensity in the ROI from the background was calculated. The results represent mean \pm standard deviation for three samples (Mannitol-sucrose ratios were $\boldsymbol{\square} 1: 0 ; \Delta 5: 2 ; \bullet 4: 3 ; \triangleleft 3: 4 ; \nabla 0: 1$ ). 
poloxamer concentration of $0.15 \% \mathrm{w} / \mathrm{v}$ was selected as optimum based on size and echogenicity for further development of RGD conjugated echogenic liposomes.

\subsubsection{Determination of Conjugation Efficiency}

One of the most stable and efficient conjugation reactions, the formation of covalent thioether bond by the reaction between sulfhydryl group and maleimide group [172, 242244] was used for conjugation of cRGD to maleimide DSPE-PEG ${ }_{2000}$. The formation of conjugate was verified by reacting cRGD and maleimide DSPE-PEG 2000 in 1:1 ratio. The conjugate was purified, and analyzed by quadrupole time-of-flight (Q-TOF) mass spectrometry using electrospray ionizer in MS only positive ion mode. The analysis of mass/charge ratio of the product ions confirmed the formation of conjugate in the reaction (Figure 2-8). For the preparation of RGD-TLCL, maleimide DSPE-PEG 2000 was incorporated in the liposome bilayer during film formation step to form Maleimide-LCL with reactive maleimide functional groups. The cRGD peptide was added to preformed liposomes with maleimide functional group on the distal end of the PEG spacer chains, and incubated at $25^{\circ} \mathrm{C}$ for the conjugation reaction. The amount of unreacted peptide present in the dialyzate after equilibrium dialysis was quantified as a measure of conjugation efficiency. Figure 2-9 shows HPLC chromatogram of dialyzate obtained from equilibrium dialysis of the liposomes immediately after conjugation reaction. The cRGD in free form elutes between 12-14 min as shown in the chromatogram for standard. The peak for $\mathrm{cRGD}$ was not detectable in the dialyzate, indicating that the reaction was nearly complete.

\subsubsection{Characteristics of GFLCL and RGD-GFTLCL}

RGD-GFTLCL was prepared using RGD-TLCL similar to the procedure for the preparation of GFLCL. The particle size of the RGD-GFTLCL was similar to GFLCL with an average diameter $\sim 150 \mathrm{~nm}$ which is favorable for longer circulation time (Figure 2-10). These liposomes can escape RES, and also benefit from passive targeting due to EPR effect. The zeta potential of both GFLCL and RGD-GFTLCL was $-36.27 \pm 2.14$ and $-32.60 \pm 0.46 \mathrm{mV}$ respectively indicating that these liposomes will have good colloidal stability. The zeta potential measurements before and after lyophilization showed a drop from $-22.8 \mathrm{mV}$ to $-36.27 \mathrm{mV}$ for GFLCL, and $-26.4 \mathrm{mV}$ to $-32.60 \mathrm{mV}$ for RGD-GFTLCL after lyophilization. This phenomenon could be attributed to ionic drift across the bilayer during lyophilization. The moisture content of the dried cake was less than $1 \% \mathrm{w} / \mathrm{v}$. The dried cake exhibited acceptable physical properties with respect to appearance, cake integrity, cake height vs volume, and ease of reconstitution. The TEM images showed that both GFLCL and RGD-GFTLCL were spherical in structure (Figure 2-11). Electron transparent regions were observed in electron dense vesicles corresponding to areas of gas localization as suggested in literature [245, 246]. The liposomes appeared similar to lipid shelled microbubbles with planar microdomains [247] separated by defects which affect mechanical properties and gas permeability [248]. The echogenicity of RGDGFTLCL was also comparable to GFLCL (Data not shown). 


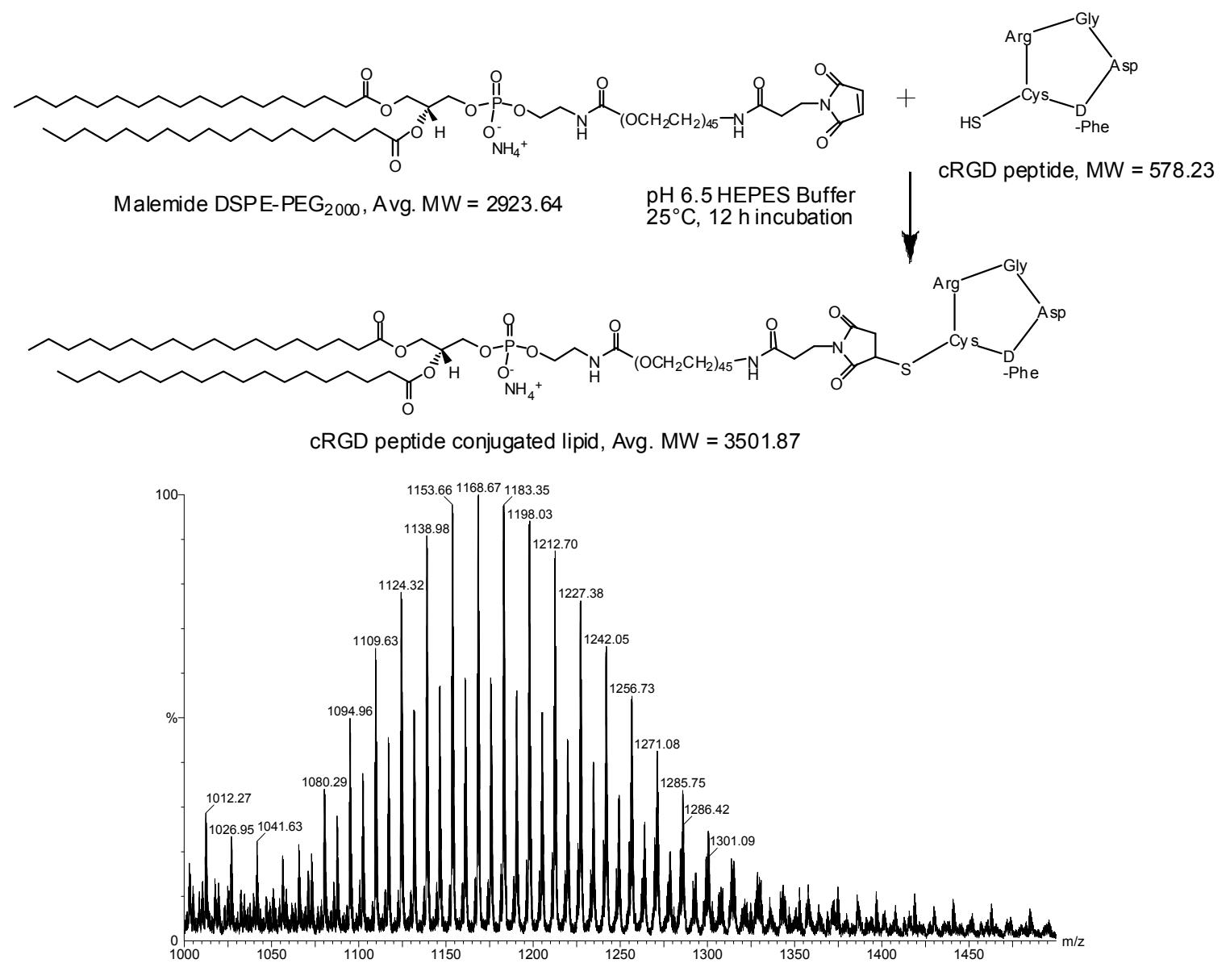

Figure 2-8. Conjugation reaction between maleimide-DSPE-PEG 2000 and cRGD

Notes. An equimolar ratio of cRGD and DSPE-PEG ${ }_{2000}$ solution in $50 \mathrm{mM}$ HEPES buffer were mixed and incubated for $12 \mathrm{~h}$. The conjugate was purified and analyzed by mass spectrometry. A segment of product ion mass spectrum is shown. 

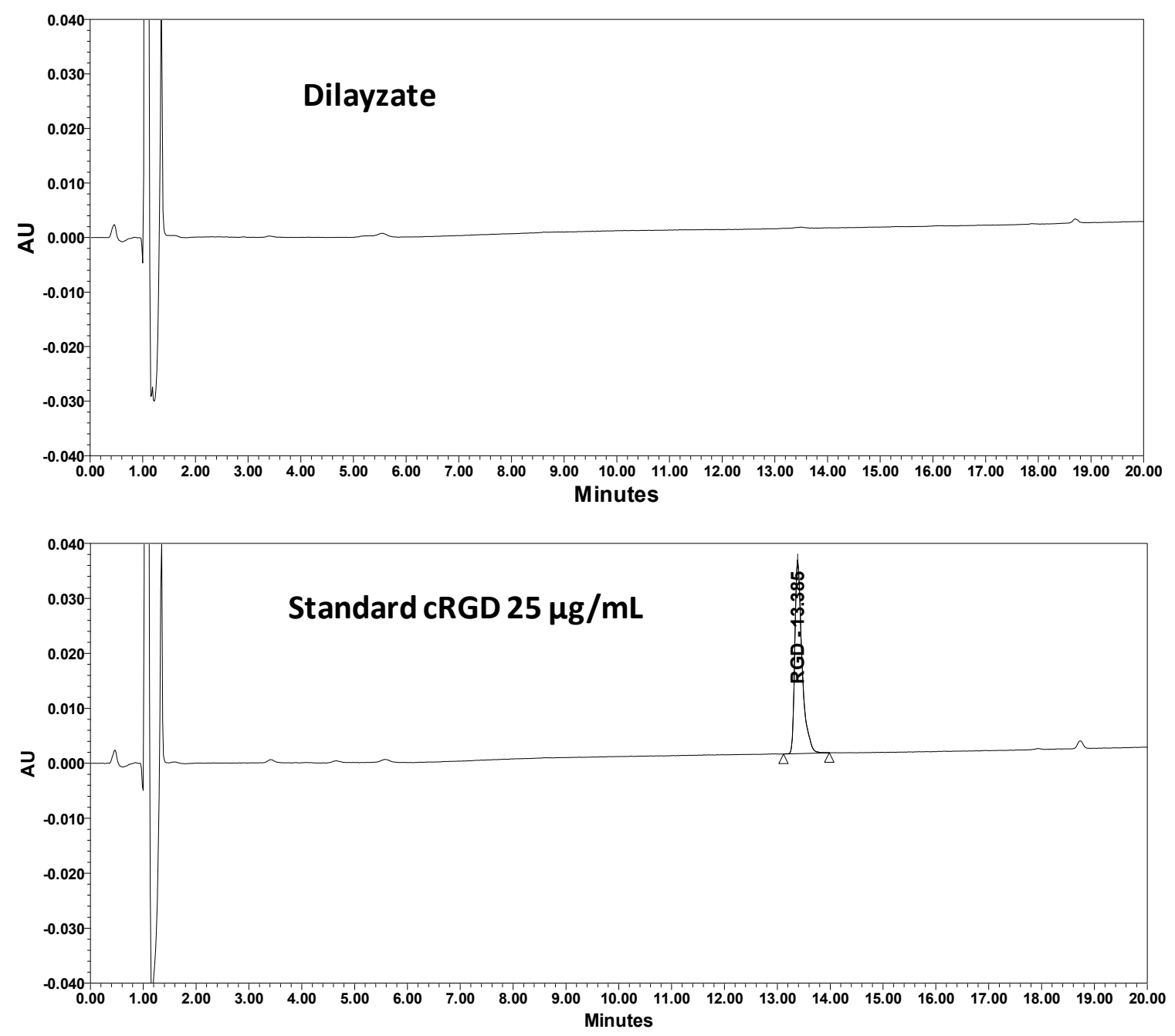

Figure 2-9. HPLC chromatogram of the dialyzate obtained from equilibrium dialysis in comparison to standard solution of $\mathrm{cRGD}$

Notes. The liposomes were dialyzed by equilibrium dialysis through 10,000 MWCO cellulose membrane. The dialyzate obtained was analyzed by HPLC (top panel). The lower panel shows the chromatogram obtained for a standard solution of cRGD. The HPLC conditions were as follows: Mobile phase - Solvent A (0.05\% v/v TFA in water) and Solvent B $(0.05 \% \mathrm{v} / \mathrm{v}$ TFA in acetonitrile) with a linear gradient of 10 to $60 \% \mathrm{~B}$ in 50 min; Column- Nov-Pak $\mathrm{C}_{18}$; Detection wavelength - $220 \mathrm{~nm}$. 


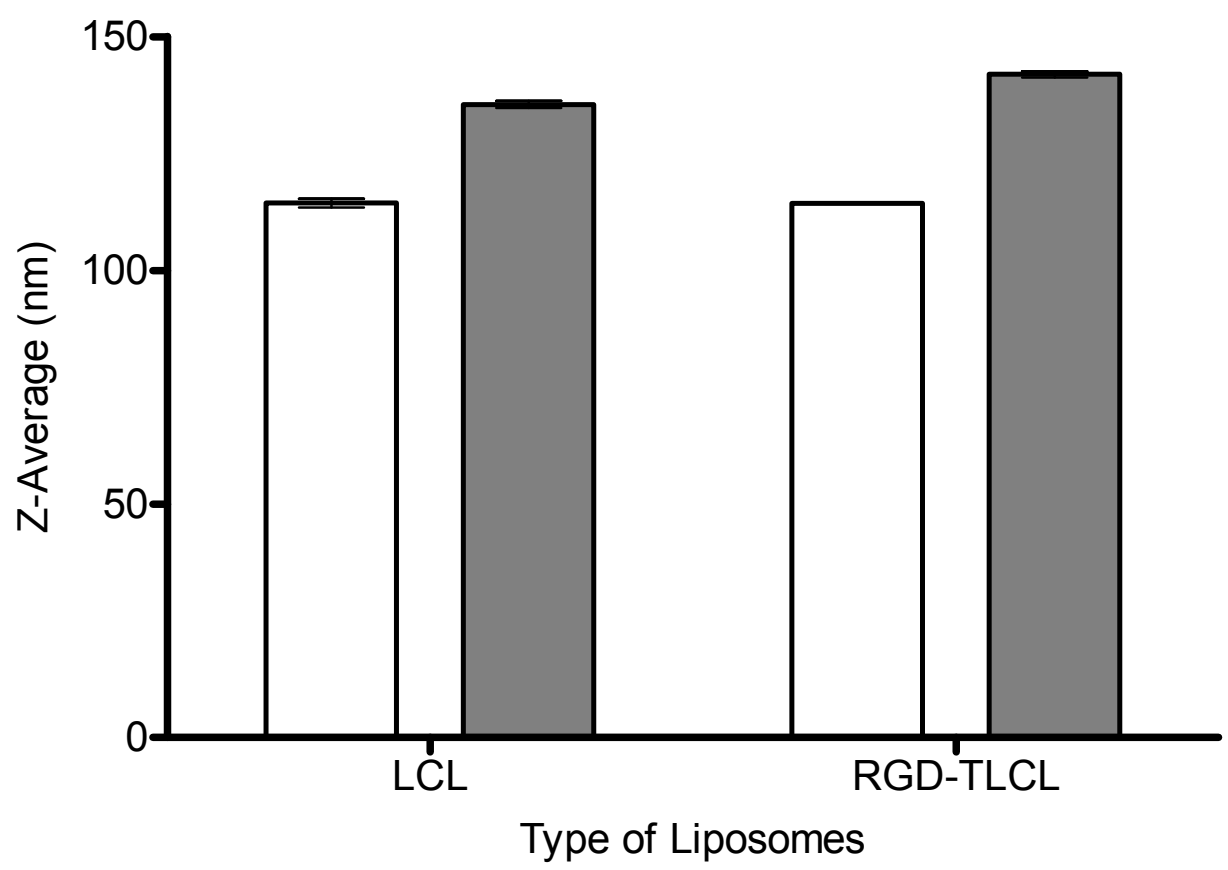

Figure 2-10. PSD of GFLCL and RGD-GFTLCL before and after lyophilization

Notes. The PSD was determined before and after lyophilization. The freeze dried gasfilled liposome samples were reconstituted with HPLC grade water for the measurement of PSD. The results indicate mean \pm standard deviation of three independent measurements ( $\square$ Before lyophilization; $\square$ After lyophilization). 


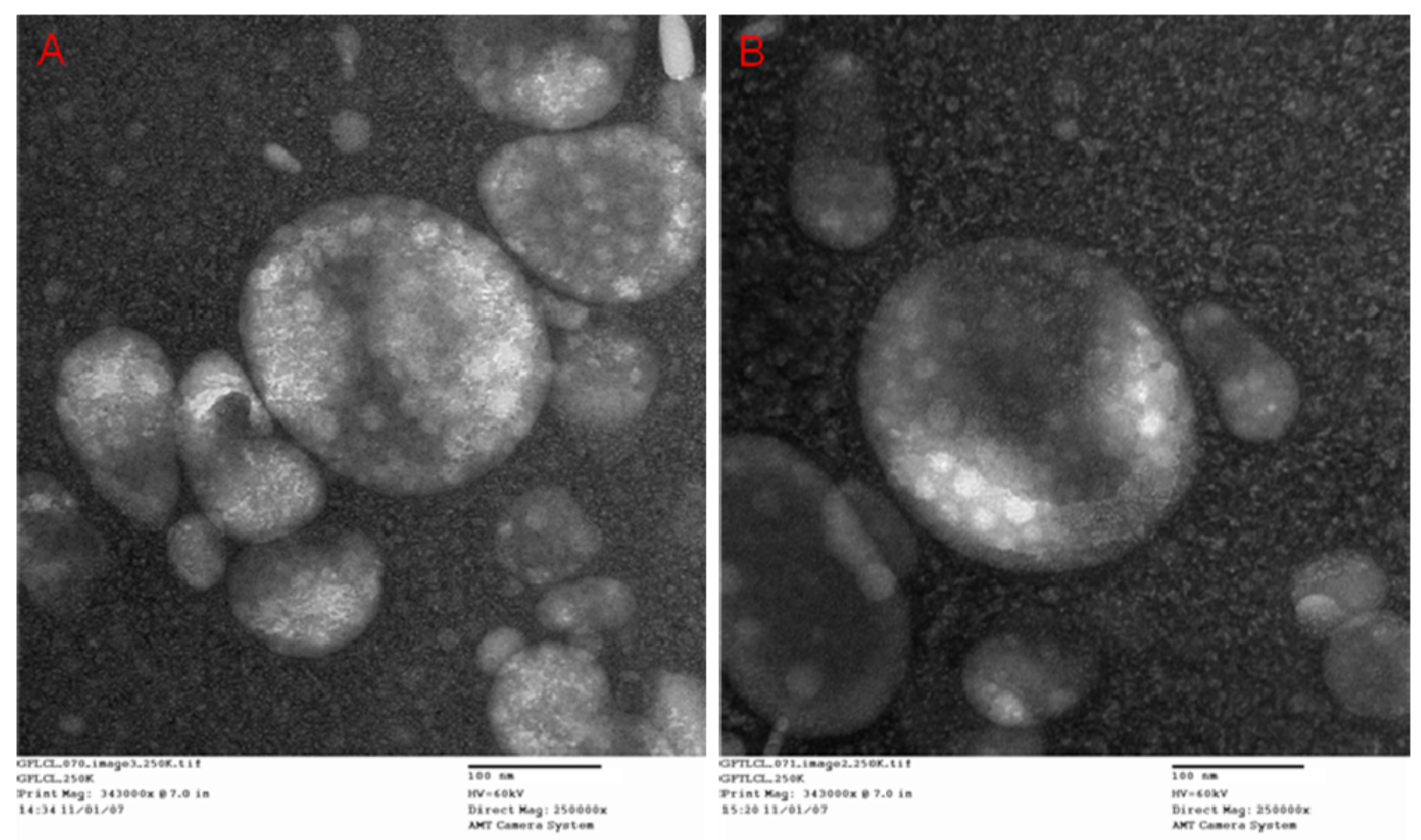

Figure 2-11. TEM images of GFLCL (A) and RGD-GFTLCL (B)

Notes. The samples were prepared by negative staining with uranyl acetate using sequential two drop technique on formvar coated copper grids. The results are shown at a magnification of 250,000x. 


\subsection{SUMMARY AND CONCLUSIONS}

The formulation and process parameters were identified and optimized in the preparation of GFLCL. The crystallization of mannitol was controlled by the inclusion of lyoprotectant sucrose so that echogenic liposomes of size less than $200 \mathrm{~nm}$, suitable for drug delivery can be prepared. The formulations containing mannitol showed over twofold higher echogenicity compared to formulations containing mannitol and sucrose. An interesting observation was that the liposomes prepared without mannitol also showed echogenicity despite negligible increase in the average diameter. The optimum formulation contained both mannitol and sucrose in a ratio $4: 3$ to meet the target requirements for size and echogenicity. Although poloxamer 188 did not show any effect on crystallization of mannitol, its steric effects improved the formulation with respect to control in size of the liposomes during lyophilization. The cRGD peptide was successfully conjugated to the PEG termini on the surface of the liposomes. The RGDGFTLCL was similar to GFLCL in size, charge, and echogenicity demonstrating that the conjugation procedure did not affect the properties of these echogenic liposomes. These echogenic liposomes can serve as a potential platform for the delivery of hydrophilic and/or lipophilic drugs for targeted drug delivery to solid tumors. The ultrasound activity can be used for visualizing these agents at the target site, and by changing the ultrasound intensity they can be disintegrated to release the encapsulated drugs. The uptake at the tumor sites can also be improved by enhanced vascular permeability mediated by ultrasound disintegration. 


\section{CHAPTER 3. OPTIMIZATION OF PACLITAXEL LOADING AND PHYSICAL STABILITY IN THE DEVELOPMENT OF LONG-CIRCULATING LIPOSOMES}

Paclitaxel is one of the most effective drugs used in chemotherapy of cancer and is a natural product isolated from bark of the Pacific Yew tree, Taxus brevifolia [8, 9]. It exhibits cytotoxic activity by promoting the assembly of microtubules from tubulin dimers and stabilizes microtubules by preventing depolymerization. The stability results in inhibition of the normal dynamic reorganization of the microtubule network that is essential for vital interphase and mitotic cellular functions [9]. Paclitaxel is marketed as Taxol $^{\circledR}$ (Bristol-Myers Squibb Company, Princeton, NJ) for the treatment of ovarian, breast, non-small cell lung cancers, and AIDS related Kaposi's sarcoma [249]. Since paclitaxel is highly insoluble in water, Taxol ${ }^{\circledR}$ was formulated with ethanol and Cremophor ${ }^{\circledR}$ EL. Although the formulation is diluted before use, the concentration of Cremophor ${ }^{\mathbb{R}} \mathrm{EL}$ is high enough to cause serious hypersensitivity reactions on parenteral administration, which require pretreatment with antihistamines and corticosteroids [15]. In addition, the use of free paclitaxel can affect normal tissues and result in systemic toxicities such as myelosuppression, and peripheral neuropathy [17]. So, it is imperative to deliver paclitaxel locally to the tumor sites to minimize systemic toxicity. In addition, failure to maintain adequate concentration at the tumor microenvironment can result in regrowth of the tumor cells and development of resistance [5].

Liposomes are biocompatible carriers with ability to incorporate both lipophilic and hydrophilic drugs [23]. Encapsulating drugs within vesicles has the potential advantage of higher drug carrying capacity compared to attaching them to single polymer chain [250]. Preparation of liposomes of paclitaxel can alter the pharmacokinetic and pharmacodynamic properties of the free drug and result in reduced toxicity and enhanced efficacy of the treatment [251]. The maximum tolerated dose of paclitaxel has been shown to increase with the liposomal formulation compared to the administration of Taxol $^{\circledR}$ [252]. The liposomal formulation of paclitaxel was also effective in delaying tumor progression in paclitaxel resistant Colon 26 tumor in mice [251, 253], suggesting that the development of liposomes opens up newer avenues to overcome drug resistance in cancer.

Conventional liposomes are cleared rapidly by the reticuloendothelial system (RES) comprised of Kupffer cells of the liver, and fixed macrophages of the spleen [80]. Clearance of liposomes by the RES is directly proportional to the vesicle size [79]. Liposomes of size $\sim 100 \mathrm{~nm}$ can escape clearance by the RES and remain in circulation for a prolonged period of time. In addition, coating the surface of the liposomes with hydrophilic polymers such as PEG can provide a shielding effect to the liposomes and prevent recognition and binding of opsonins [88]. Therefore, these liposomes can escape clearance by the RES and remain in circulation for a longer period of time. If the liposomes remain in circulation for a longer time, the possibility of their passive accumulation in the tumors by enhanced permeability and retention (EPR) effect will be improved. Even with PEG coating to minimize the clearance by the RES, the cutoff size for the liposomes for long circulation remain between 150 and $200 \mathrm{~nm}$ [28]. 
Loading paclitaxel in the bilayer of the liposomes, and retaining the encapsulated drug during storage requires special consideration in product development because the encapsulated drug tends to precipitate at higher loading. Paclitaxel is incorporated into the acyl chain portion of the liposome bilayer [254]. At lower concentrations, it partitions into the upper bilayer domain towards the aqueous interface. When the concentration is increased, it partitions into to deeper parts of the bilayer. Higher concentrations can induce self aggregation which can act as nucleation for precipitation of paclitaxel as needle shaped crystals. Partitioning of paclitaxel in the bilayer of liposomes induces conformational changes which are different for saturated and unsaturated lipids [255]. Thermotropic phase behavior of phospholipids is altered inducing fluidity in the gel phase of saturated lipids, and slight rigidity in unsaturated lipids. It also affects bilayer spacing of planar phospholipids in lipid specific, and concentration dependant manner [256]. Thus, drug-lipid interaction plays a major role in determining the physical stability of the liposomes [257]. It has been suggested that a tradeoff exists between the drug content and duration of stability in aqueous dispersion [252]. Even though high drug loading has been reported with certain lipid compositions [249, 258, 259], the physical stability of the liposomes varied with each composition. Apart from that, inclusion of PEGylated lipids has been shown to reduce drug loading and physical stability of liposomes [258]. The extent of paclitaxel loading in the liposomes, and the stability of such liposomes is dependent on the nature and concentration of phospholipids, and druglipid interaction determined by the drug-lipid ratio. So, there is a need to optimize these parameters to achieve adequate stability of the liposomes. The objective of this work is to determine, and optimize the factors influencing drug loading, and physical stability of paclitaxel-loaded long-circulating liposomes (Pac-LCL).

\subsection{MATERIALS AND METHODS}

\subsubsection{Materials}

Paclitaxel was procured from 21CEC Pharmaceuticals (Bradenton, FL). The phospholipids used in this work were all of research grade. Egg phosphatidylcholine (EPC), hydrogenated soy phosphatidylcholine (HSPC) and 1,2-distearoyl-sn-glycero-3phoshoethanolamine-N-(poly(ethylene glycol)2000) (DSPE-PEG 2000 ) were obtained from Northern Lipids (Burnbay, BC, Canada). Cholesterol and $\alpha$-tocopherol were purchased from Sigma (St. Louis, MO). HPLC grade water, acetonitrile, and methanol were obtained from Fisher Scientific (Fair Lawn, NJ). HPLC grade chloroform was purchased from Acros Organics (Morris Plains, NJ). N-2-hydroxyethylpiperazine-N'-2ethanesulfonic acid (HEPES) buffer, $1 \mathrm{M}$ was obtained from Mediatech (Manassas, VA). All other chemicals and reagents were of analytical grade and used without further purification or characterization. 


\subsubsection{Preparation of Paclitaxel-Loaded Long-Circulating Liposomes (Pac-LCL)}

The Pac-LCL was be prepared by the lipid film hydration-extrusion method using HSPC, DSPE-PEG 2000 , cholesterol, and/or EPC. The lipid film was prepared by dissolving paclitaxel, phospholipids, cholesterol, and /or $\alpha$-tocopherol in 9:1 chloroformmethanol. The solution thus obtained was evaporated in a round bottom flask using rotary evaporator under reduced pressure at $45^{\circ} \mathrm{C}$ for $4-6 \mathrm{~h}$. The dry film was hydrated with $50 \mathrm{mM}$ HEPES buffer $\mathrm{pH} 6.5$ at $65^{\circ} \mathrm{C}$ for $45-60 \mathrm{~min}$. The multilamellar vesicles (MLVs) thus obtained were sized by extrusion through stacked polycarbonate membrane filters of varying pore diameters under high pressure to obtain small unilamellar vesicles (SUVs). The SUVs were characterized for particle size distribution, zeta potential, paclitaxel loading and morphology. The pore diameter of filters, pressure, and number cycles used in the extrusion process were optimized to obtain an average particle size of $100 \pm 20 \mathrm{~nm}$.

\subsubsection{Determination of Paclitaxel Loading Efficiency}

The loading efficiency of paclitaxel in the liposomes was determined by minicolumn centrifugation method using Sephadex G-25 macro spin columns (Harvard apparatus, Holliston, MA) similar to the method described by Zhang et al [249] with modifications. Briefly, the Sephadex G-25 particles in the column were suspended in $0.1 \% \mathrm{w} / \mathrm{v}$ TFA and was allowed to swell for 15 minutes. The columns were centrifuged at $1500 \mathrm{~g}$ at $25^{\circ} \mathrm{C}$ for 4 min using IEC CL31R Multispeed Centrifuge (Thermo Scientific, Asheville, NC) to remove TFA. About $100 \mu \mathrm{L}$ of placebo LCL was added and centrifuged to saturate the column. The column saturation with placebo liposomes was repeated for a total of three times. The gel columns were placed in fresh collection tubes for use with drug-loaded liposomes. The Pac-LCL samples were diluted with $50 \mathrm{mM}$ HEPES buffer $\mathrm{pH}$ 6.5. About $100 \mu \mathrm{L}$ of the diluted sample was added to the gel column, and centrifuged for 4 minutes. The eluate in the collection tube, containing only entrapped paclitaxel was analyzed by RPHPLC. The encapsulation efficiency was calculated from total paclitaxel determined before passing through Sephadex G-25, using the formula, Entrapment efficiency = (Entrapped paclitaxel x 100)/Total paclitaxel).

\subsubsection{Quantification of Paclitaxel from Liposomes}

The total and entrapped paclitaxel in the liposomes was quantified by RPHPLC method. The HPLC system comprised of Waters 600 Controller, Waters 717 Plus Autosampler, and Waters 2996 Photodiode Array Detector (Waters Corporation, Milford, MA) was used. The RPHPLC analysis was performed using a Nova-Pak $\mathrm{C}_{18}$ column (3.9 x $150 \mathrm{~mm}, 4 \mu \mathrm{m}$ ) (Waters Corporation, Milford, MA). An isocratic elution was performed using acetonitrile-water in a ratio $55: 45 \mathrm{v} / \mathrm{v}$ at a flow rate of $1 \mathrm{~mL} / \mathrm{min}$, and detection wavelength of $230 \mathrm{~nm}$. The liposomes were digested with absolute ethanol, and suitably diluted before injection. 


\subsubsection{Determination of Particle Size and Zeta Potential}

The average diameter (Z-avg) of the Pac-LCL was determined using dynamic light scattering using Zetasizer Nano ZS (Malvern Instruments, Westborough, MA) equipped with $4.5 \mathrm{~mW}$ diode laser as a source of light operating at $670 \mathrm{~nm}$. Particle scattered photons were detected at an angle of $173^{\circ}$. The samples were suitably diluted with HPLC grade water for the determination of Z-avg. Three independent measurements were performed for each sample. Zeta potential was determined from electrophoretic mobility using samples suitably diluted with HPLC grade water.

\subsubsection{Stability Evaluation of Pac-LCL}

Stability of Pac-LCL was evaluated at two stages. Initially, the stability was evaluated for 7-10 days for the optimization of the liposome composition. The Pac-LCL samples were stored in $7 \mathrm{~mL}$ glass scintillation vials at $5 \pm 3^{\circ} \mathrm{C}$. Aliquots were withdrawn at specific intervals. The entrapped paclitaxel was determined by minicolumn centrifugation method followed by analysis of the eluate by RPHPLC method. In the stability evaluation stage, the optimized formulation was stored in $2 \mathrm{cc}$ amber glass vials at $5 \pm 3^{\circ} \mathrm{C}$ for three months. Samples were withdrawn at specific intervals and analyzed for total and entrapped paclitaxel, entrapment efficiency, PSD, and zeta potential.

\subsubsection{Characterization of Morphology of Pac-LCL}

Morphology of the Pac-LCL was characterized by transmission electron microscopy (TEM) using a negative staining technique. Sequential two droplet method was used for staining liposome samples with $2 \% \mathrm{w} / \mathrm{v}$ uranyl acetate on 400 -mesh formvar support film on copper specimen grid (Electron Microscopy Sciences, Hartfield, PA). The TEM images were acquired using JEOL 2000EX transmission electron microscope (JEOL USA, Inc., Peabody, MA) equipped with high resolution digital camera.

\subsection{RESULTS AND DISCUSSION}

\subsubsection{Optimization of Paclitaxel Loading}

The target concentration in the paclitaxel liposome dispersion was set at $1.0-1.5$ $\mathrm{mg} / \mathrm{mL}$ with a size of $100 \pm 20 \mathrm{~nm}$. The initial composition for the liposomes was HSPC: DSPE-PEG $_{2000}$ : cholesterol in a ratio of 45: 5: 50, and a total lipid concentration of 10 $\mathrm{mM}$. The entrapment efficiency of paclitaxel was calculated by minicolumn centrifugation method using Sepadex G-25 columns. The columns were pretreated with placebo liposomes three times to completely saturate the column so that non-specific adsorption and subsequent loss of paclitaxel-loaded liposomes can be minimized. The total and entrapped paclitaxel in the liposome formulation was calculated from standard 
curve which was liner between 0.256 to $20 \mu \mathrm{g} / \mathrm{mL}$ with a coefficient of determination $\left(\mathrm{r}^{2}\right)$ value of 0.9999 .

The effect of total phospholipids on paclitaxel loading is shown in Figure 3-1. As expected, an increase in total phospholipids resulted in an increase in the amount of paclitaxel per $\mathrm{mL}$ of liposomes. But, the entrapment efficiency was low with a total entrapped paclitaxel concentration of $\sim 4.0 \mu \mathrm{g} / \mathrm{mL}$ at the highest concentration tested for phospholipids. Inclusion of cholesterol in the bilayer of liposomes has been shown to improve the rigidity of the bilayer, and thereby, improve in vitro and in vivo stability of the liposomes [59, 61, 64]. It also reduces leakage of encapsulated contents from the liposomes. Cholesterol was included in the formulation to improve the rigidity, and to minimize drug leakage. Figure 3-2 shows the effect of cholesterol on paclitaxel loading in the LCL formulation. Reducing cholesterol content from 50 to 30 moles \% did not show any change in the paclitaxel loading. But, a sharp increase in loading was observed at 10 moles $\%$ of cholesterol with an entrapped drug content of $\sim 100 \mu \mathrm{g} / \mathrm{mL}$. The negative effect of cholesterol on paclitaxel loading was consistent with similar reports in literature [249, 258]. It has been suggested that paclitaxel competes with cholesterol for hydrophobic space in the bilayer of liposomes [249]. If more molecules of cholesterol are present, there would be less space for paclitaxel to occupy. Cholesterol can restrict the flexibility of hydrocarbon chains and prevent penetration of paclitaxel into the bilayer [249]. In contrast, inclusion of cholesterol even at lower concentrations in PEGylated liposomes, have shown a positive effect in improving serum stability by preventing exchange of phospholipids with high density lipoproteins. So the lowest concentration of cholesterol was selected for further studies.

High rigidity of HSPC, a saturated phospholipid, can restrict the loading of paclitaxel into the bilayer of liposomes. So we evaluated the effect of EPC, an unsaturated phospholipid, using different ratios on paclitaxel loading (Figure 3-3). With a 1: 1 ratio of HSPC-EPC, the paclitaxel loading increased to $\sim 800 \mu \mathrm{g} / \mathrm{mL}$. The results were consistent with literature reports on paclitaxel loading using unsaturated phospholipids [171, 249, 260, 261]. Unsaturated lipids have a low gel to liquid crystalline transition temperature $\left(\mathrm{T}_{\mathrm{m}}\right)$, and form liposomes which are more flexible in nature to accommodate lipophilic drugs [249]. Though inclusion of unsaturated lipids have a positive influence, they cannot be used solely to prepare liposomes because of problems related to aggregation of paclitaxel-loaded liposomes made of PC alone [252]. Further increases in EPC showed only a minimal increase in drug loading. Nevertheless, paclitaxel precipitated from all the liposome compositions within few days of storage at $5 \pm 3^{\circ} \mathrm{C}$ suggesting that further refinement of the formulation is necessary. When the total lipids content of HSPC-EPC ratio 1:3 was increased to $100 \mathrm{mM}$, the entrapped paclitaxel content increased to $\sim 1.9 \mathrm{mg} / \mathrm{mL}$ but precipitation was observed within few days of storage. A further increase in the lipid concentration was not feasible because the multilamellar vesicles (MLVs) formed a gel during hydration stage. High loading being achieved, the optimum amount paclitaxel loading that result in physically stable formulation needs to be determined. 


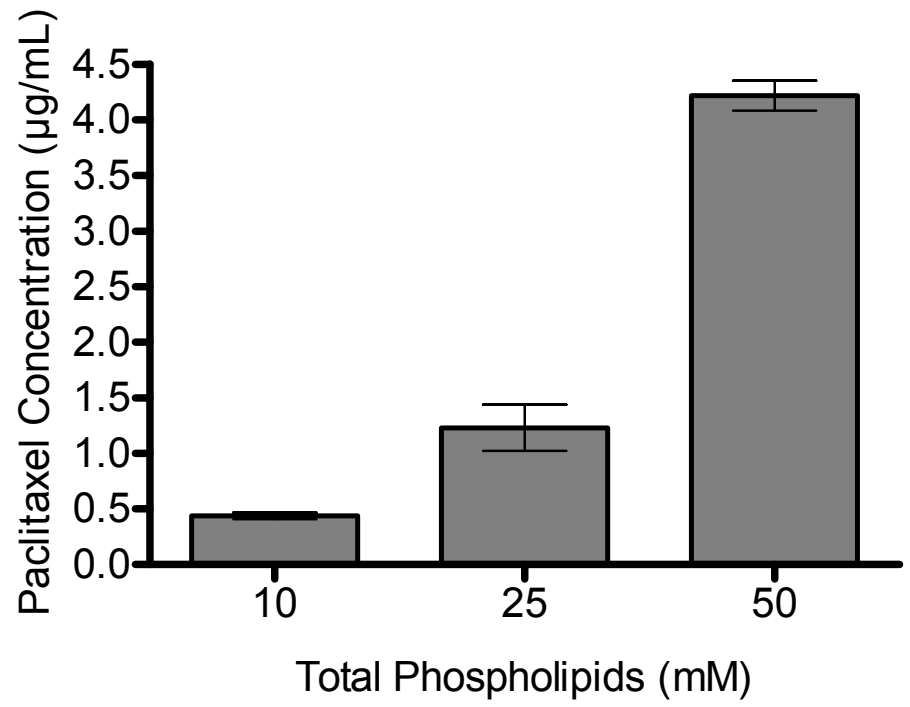

Figure 3-1. Effect of total phospholipids on paclitaxel loading

Notes. The liposome composition was HSPC: DSPE- $\mathrm{PEG}_{2000}$ : cholesterol in a ratio of 45: 5: 50. The liposomes were subjected to minicolumn centrifugation in Sephadex G-25 macro spin columns to separate free paclitaxel from total paclitaxel. The eluate containing entrapped paclitaxel was digested with ethanol, and quantified by RPHPLC method. Two injections were made for each sample, and the average was calculated. Three Sephadex columns were prepared for each composition and the results indicate the mean \pm standard deviation for three gel filtration columns. 


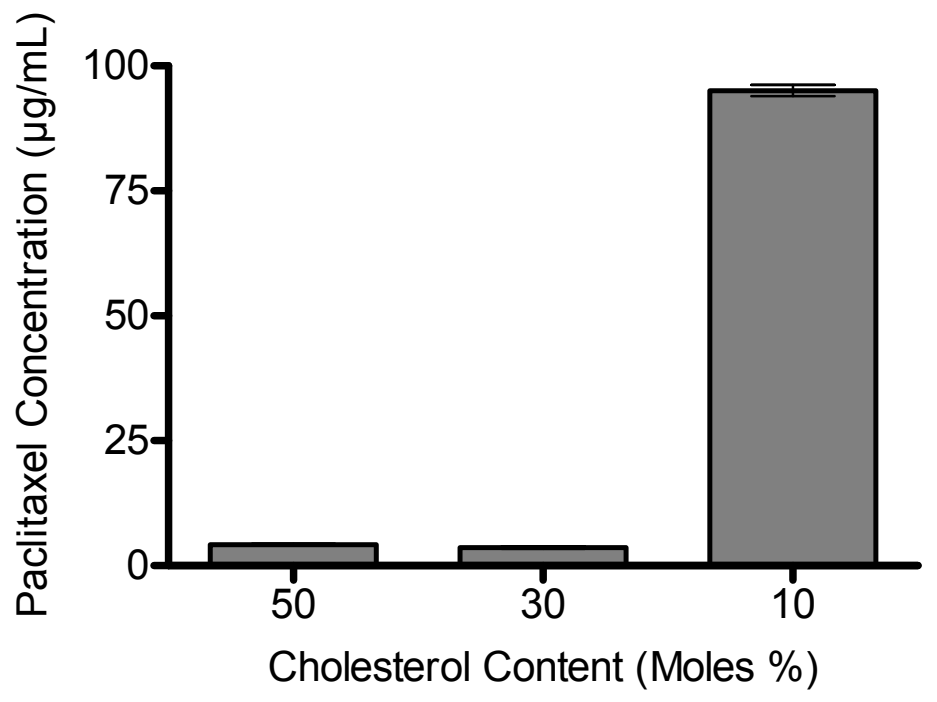

Figure 3-2. Effect of cholesterol concentration on paclitaxel loading

Notes. The liposome composition was HSPC: DSPE-PEG ${ }_{2000}$ : cholesterol in a ratio of $\mathrm{x}: 5$ : $\mathrm{x}$ moles $\%$ with a total lipid concentration of $50 \mathrm{mM}$. The liposomes were subjected to minicolumn centrifugation in Sephadex G-25 macro spin columns to separate free paclitaxel from total paclitaxel. The eluate containing entrapped paclitaxel was digested with ethanol, and quantified by RPHPLC method. Two injections were made for each sample, and the average was calculated. Three Sephadex columns were prepared for each composition and the results indicate the mean \pm standard deviation for three gel filtration columns. 


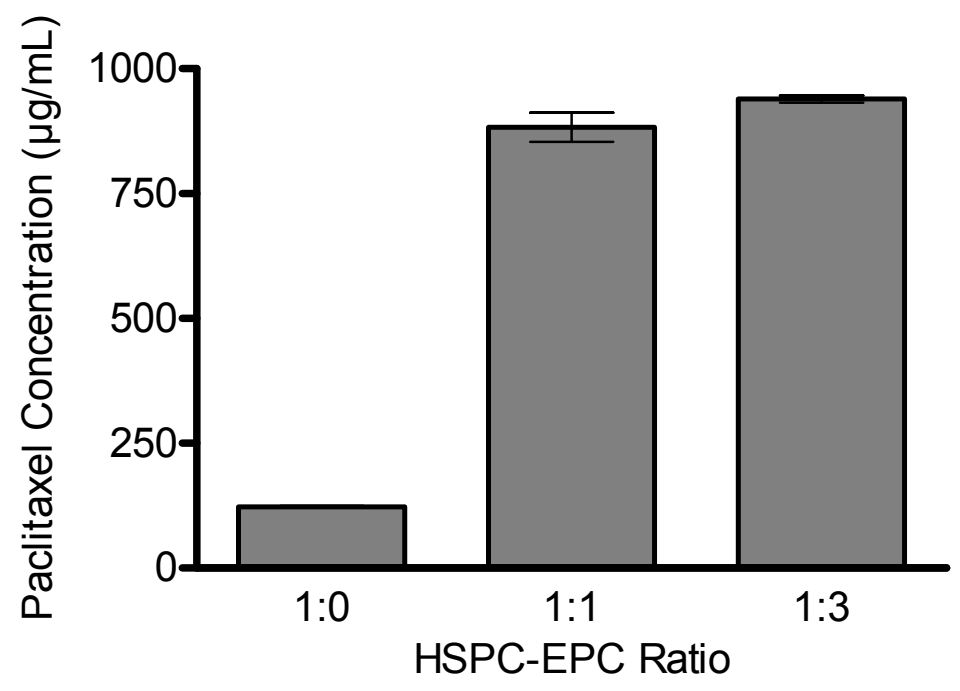

Figure 3-3. Effect of saturated-unsaturated lipid ratio on paclitaxel loading

Notes. The liposome composition was HSPC: EPC: DSPE-PEG 2000 : cholesterol with a total lipid concentration of $50 \mathrm{mM}$. The moles $\%$ of DSPE-PEG 2000 and cholesterol were maintained constant at $5 \%$ and $10 \%$ respectively. The liposomes were subjected to minicolumn centrifugation in Sephadex G-25 macro spin columns to separate free paclitaxel from total paclitaxel. The eluate containing entrapped paclitaxel was digested with ethanol, and quantified by RPHPLC method. Two injections were made for each sample, and the average was calculated. Three Sephadex columns were prepared for each composition and the results indicate the mean \pm standard deviation for three gel filtration columns. 


\subsubsection{Optimization of Physical Stability}

Drug-lipid interaction is a major determining factor of physical stability of the liposomes and is dependent on the concentration of drug and phospholipids [257]. Higher to drug-lipid ratio can minimize the total amount of phospholipids administered and thus reduce toxicities associated with high lipid doses. On the other hand, the ratio needs to be optimum to attain adequate physical stability. Thus, the optimum formulation is the one which has the highest drug-lipid ratio achievable for physically stable liposomes. A paclitaxel-lipid ratio of 1:30 or less has been used to develop formulations with different phospholipid compositions with good loading efficiency [249, 252, 258]. We evaluated the effect of drug-lipid ratio on paclitaxel loading in LCL at different ratios. With increasing drug-lipid ratio, an increase in drug loading was observed (Figure 3-4) with a maximum loading obtained at the ratio of 1:30. Further increase in drug-lipid ratio to 1:20 resulted in a decrease in loading due to precipitation (Data not shown). This was consistent with literature reports suggesting that the ratios above 1:30 can result in aggregate formation that can act as nucleation for precipitation [254]. The LCL containing three different drug-lipid ratios were stored in scintillation vials at $5 \pm 3^{\circ} \mathrm{C}$ to evaluate the physical stability of these liposomes. At specific intervals, aliquots were withdrawn from these vials after vortexing to ensure uniform mixing. The liposomes were passed through Sephadex G-25 gel column by minicolumn centrifugation method to separate the free drug from the dispersion. The entrapped paclitaxel in these LCL formulations were plotted against time (Figure 3-5) to evaluate the physical stability. The entrapped paclitaxel decreased to nearly $20 \%$ of the initial concentration in five days for the drug-lipid ratio of 1:30. The formulation with 1:45 also showed a steady decline in entrapped paclitaxel through the 10 days of observation. The ratio of 1:60 was stable for up to 10 days. Even though high loading was achieved with the drug-lipid ratios 1:30, and 1:45, they were not physically stable in this bilayer composition. This could be attributed to over saturation of the bilayer with paclitaxel in the liposomes at higher temperatures above the storage temperature. On returning to the storage temperature, paclitaxel in the oversaturated liposomes started to precipitate. The precipitate acted as nucleation and induced further precipitation of remaining paclitaxel reducing the encapsulated paclitaxel content to a lower level even below that was seen with the ratio 1:60. The liposome bilayer composition was further modified to minimize the negative factors that affect paclitaxel loading. HSPC-EPC ratio was changed to 1:5, and cholesterol concentration was reduced to 5 moles $\%$. The amount of entrapped drug for a given drug-lipid ratio increased for these formulations. The effect of various drug-lipid ratios on the physical stability of the improved composition was evaluated at $5 \pm 3^{\circ} \mathrm{C}$ for one week, and the data is presented in Figure 3-6. Similar to the previous observations the drug to lipid ratio of 1:30 showed a decrease in entrapped paclitaxel but at a slower rate. Other ratios were stable for one week. The intermediate stable drug-lipid ratio of 1:60 was selected as the optimum formulation for three-month stability evaluation. 


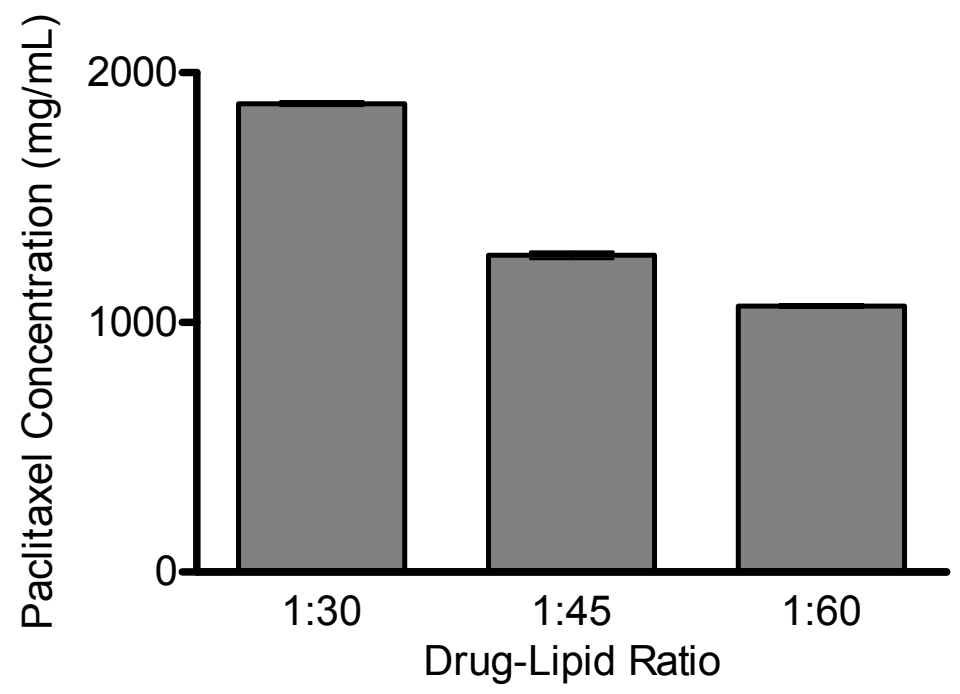

Figure 3-4. Effect of drug-lipid ratio on paclitaxel loading

Notes. The liposome composition was HSPC: EPC: DSPE-PEG ${ }_{2000}$ : cholesterol (21.25: 63.75: 5: 10) with a total lipid concentration of $100 \mathrm{mM}$. The liposomes were subjected to minicolumn centrifugation in Sephadex G-25 macro spin columns to separate free paclitaxel from total paclitaxel. The eluate containing entrapped paclitaxel was digested with ethanol, and quantified by RPHPLC method. Two injections were made for each sample, and the average was calculated. Three Sephadex columns were prepared for each composition and the results indicate the mean \pm standard deviation for three gel filtration columns. 


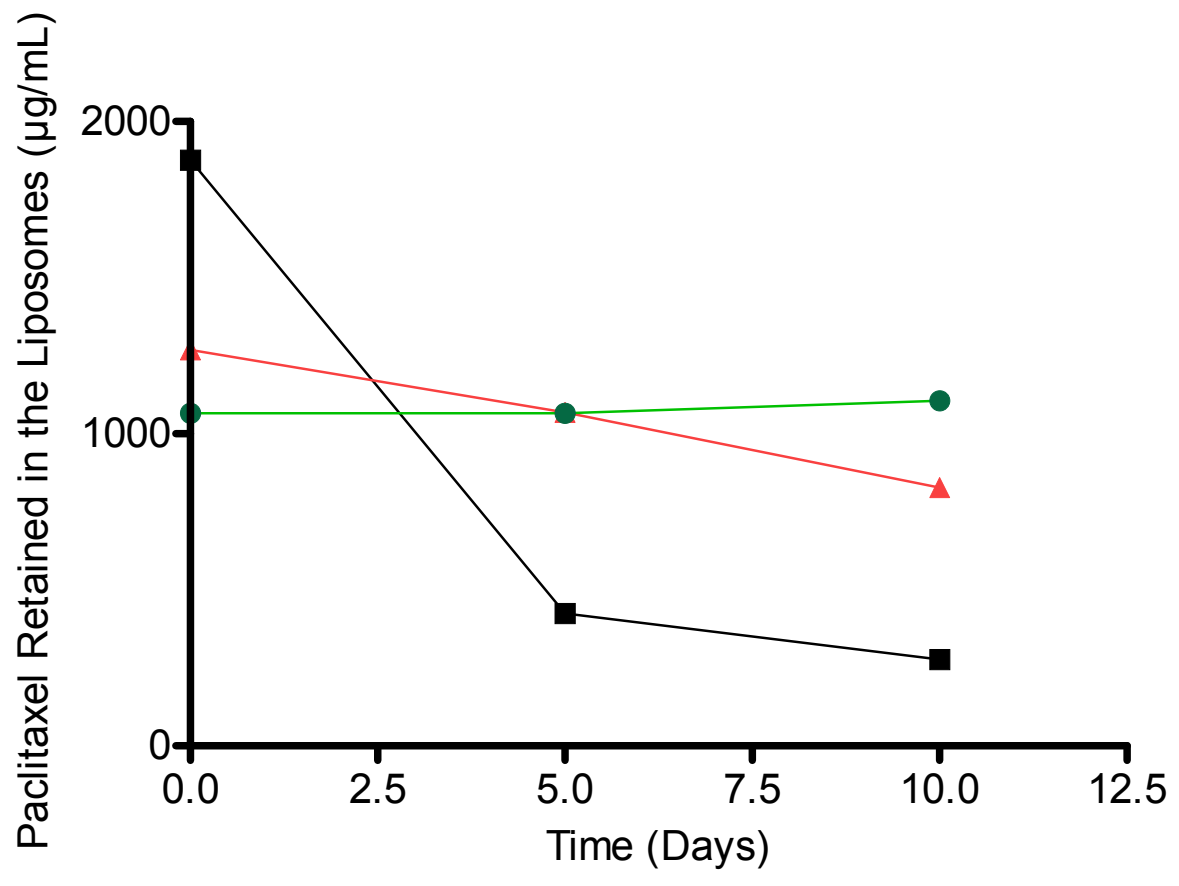

Figure 3-5. Effect of drug-lipid ratio on physical stability of Pac-LCL

Notes. The liposome composition was HSPC: EPC: DSPE-PEG 2000 : cholesterol (21.25: 63.75: 5: 10) with a total lipid concentration of $100 \mathrm{mM}$. The liposomes were stored at $5 \pm 3^{\circ} \mathrm{C}$ in scintillation vials. Aliquots were withdrawn at specific intervals and subjected to minicolumn centrifugation in Sephadex G-25 macro spin columns to separate free paclitaxel from total paclitaxel. The eluate containing entrapped paclitaxel was digested with ethanol, and quantified by RPHPLC method. Two injections were made for each sample, and the average was calculated. Three Sephadex columns were prepared for each composition and the results indicate the mean \pm standard deviation for three gel filtration columns (Drug-lipid ratios are $1: 30 ;-1: 45 ; \bullet 1: 60$ ). 


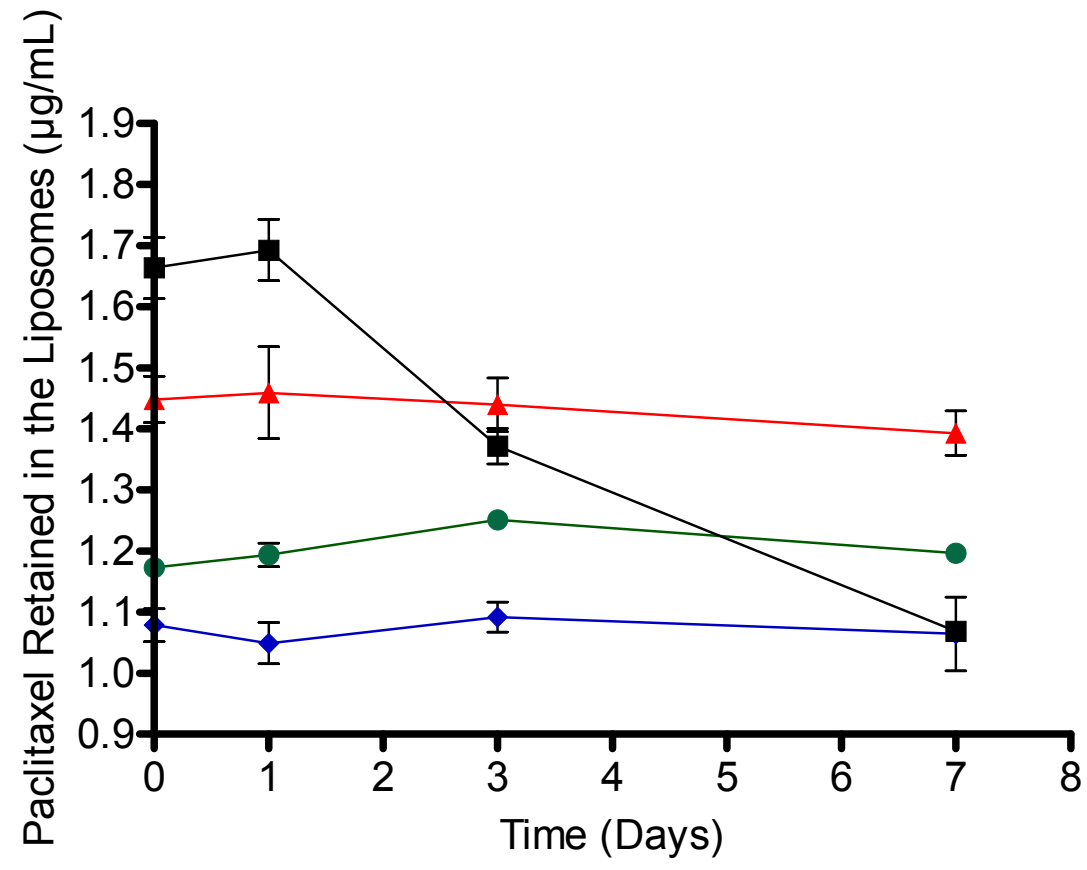

Figure 3-6. Effect of drug-lipid ratio on physical stability of improved Pac-LCL

Notes. The liposome composition was HSPC: EPC: DSPE-PEG 2000 : cholesterol (15: 75: 5: 5) with a total lipid concentration of $100 \mathrm{mM}$. The liposomes were stored at $5 \pm$ $3^{\circ} \mathrm{C}$ in scintillation vials. Aliquots were withdrawn at specific intervals and subjected to minicolumn centrifugation in Sephadex G-25 macro spin columns to separate free paclitaxel from total paclitaxel. The eluate containing entrapped paclitaxel was digested with ethanol, and quantified by RPHPLC method. Two injections were made for each sample, and the average was calculated. Three Sephadex columns were prepared for each composition and the results indicate the mean \pm standard deviation for three gel filtration columns (Drug-lipid ratios are $1: 30 ;-1: 45 ; \bullet 1: 60 ; 1: 75$ ). 


\subsubsection{Optimization of the Extrusion Process}

The extrusion process used in the preparation of LCL to reduce the vesicle size can affect drug loading depending upon the number of extrusion cycles and the extrusion pressure. The effect of number of extrusion cycles on PSD, and entrapment efficiency is presented in Figure 3-7. The results are shown for the drug-lipid ratio of 1:30, and polycarbonate membrane filter combination of $200 \mathrm{~nm}$ stacked over $100 \mathrm{~nm}$. As expected, the size of the liposomes decreased with increasing number of extrusion cycles. There was no significant decrease in the entrapped paclitaxel up to five extrusion cycles. A slight decrease in entrapment was observed with 10 extrusion cycles but it was within the target loading desired. The effect of extrusion pressure is presented in Figure 3-8. The particle size decreased proportionately with increase in extrusion pressure. The entrapment efficiency did not show any significant change with increasing extrusion pressure although there was a slight decrease in loading observed at extrusion pressure of 300 PSI. The understanding about the process helped in reaching the target size of $100 \pm$ $20 \mathrm{~nm}$ with a paclitaxel loading of $1-1.3 \mathrm{mg} / \mathrm{mL}$ with changes in polycarbonate filter combinations, extrusion pressure, and number of extrusion cycles. The TEM images of the Pac-LCL formulation showed that these vesicles were spherical in structure with a diameter of $100 \pm 20 \mathrm{~nm}$ (Figure 3-9).

\subsubsection{Stability Evaluation of Pac-LCL Dispersion}

The stability of the Pac-LCL dispersion was evaluated at $5 \pm 3{ }^{\circ} \mathrm{C}$ in light protected container for three months. The stability data presented in Figure 3-10 shows that the liposomes maintained their size, charge, and integrity through the evaluation period. A slight decrease was observed in paclitaxel retention in the first few days. This could be attributed to partitioning of the drug form the bilayer of liposomes to the dispersion medium to maintain equilibrium. Once the equilibrium is reached, the paclitaxel retention remained constant.

\subsection{SUMMARY AND CONCLUSIONS}

The loading of paclitaxel in the liposomes can vary depending upon number of factors including the nature and concentration of lipids in the bilayer, the amount of drug, and the process used in the preparation of liposomes. Even though high loading can be achieved, the physical instability of the liposomes in the dispersion is a challenging issue and needs to be addressed to ensure long-term stability during storage. The factors affecting drug loading have been identified and optimized to improve the loading efficiency. A proportionate increase in loading was observed with increase in the total phospholipid content. Cholesterol and saturated lipid content in the bilayer showed a negative influence on paclitaxel loading. Reducing the concentration of cholesterol showed a significant increase in paclitaxel loading. The increase in loading was further augmented by the inclusion of EPC, an unsaturated lipid to replace a part of HSPC, a saturated lipid. The increased flexibility of the acyl chains of the unsaturated lipids 


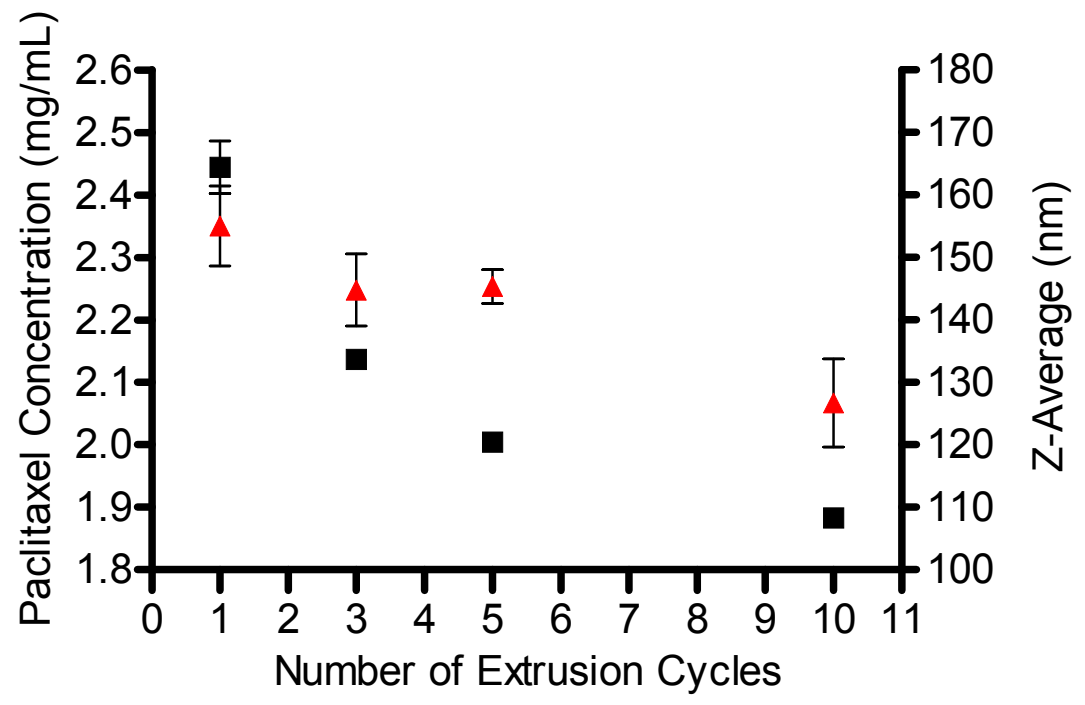

Figure 3-7. Effect of number of extrusion cycles on PSD and paclitaxel loading

Notes. Liposomes were sized by extrusion process and the samples were taken at different number of cycles and evaluated for entrapped paclitaxel and PSD. For entrapped paclitaxel determination, the eluate from gel filtration through Sephadex G-25 was digested with ethanol, and quantified by RPHPLC method. Two injections were made for each sample, and the average was calculated. Three Sephadex columns were prepared for each stage of evaluation in the extrusion process. The results indicate the mean \pm standard deviation for three gel filtration columns. For PSD, the results indicate mean \pm standard deviation of three independent measurements at each stage of evaluation ( PSD; $\Delta$ Paclitaxel loading). 


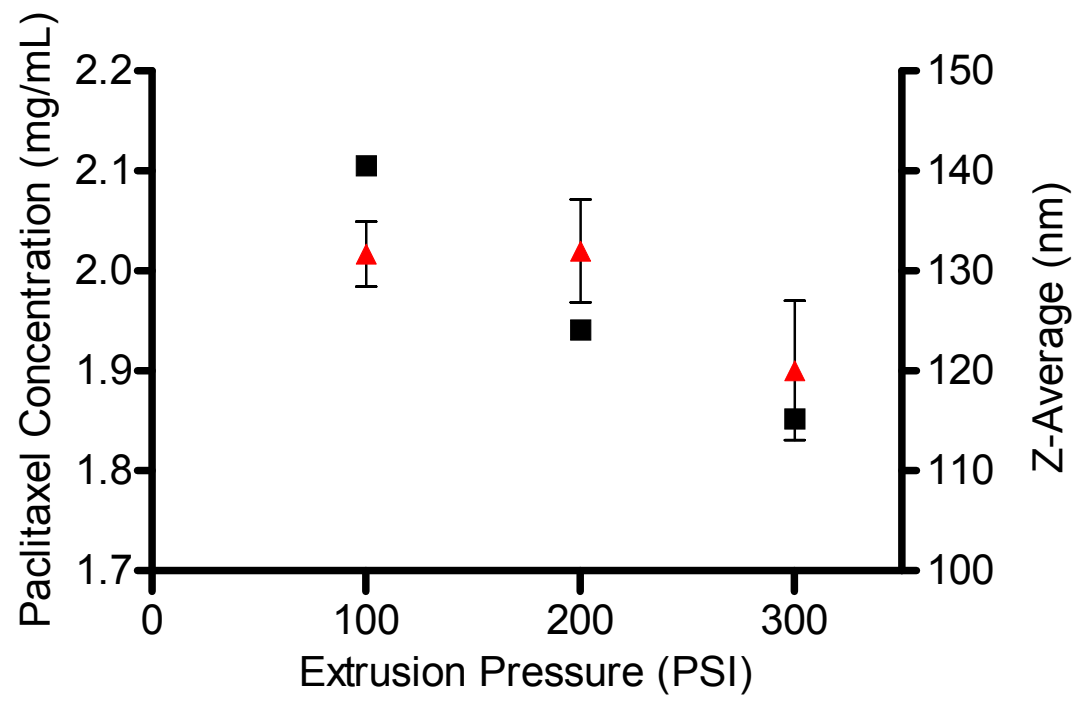

Figure 3-8. Effect of extrusion pressure on PSD and paclitaxel loading

Notes. Liposomes were sized by extrusion process using different extrusion pressures, and evaluated for entrapped paclitaxel and PSD. For entrapped paclitaxel determination, the eluate from gel filtration through Sephadex G-25 was digested with ethanol, and quantified by RPHPLC method. Two injections were made for each sample, and the average was calculated. Three Sephadex columns were prepared for each extrusion pressure. The results indicate the mean \pm standard deviation for three gel filtration columns. For PSD, the results indicate mean \pm standard deviation of three independent measurements at extrusion pressure ( $\boldsymbol{\sim}$ PSD; $\Delta$ Paclitaxel loading). 


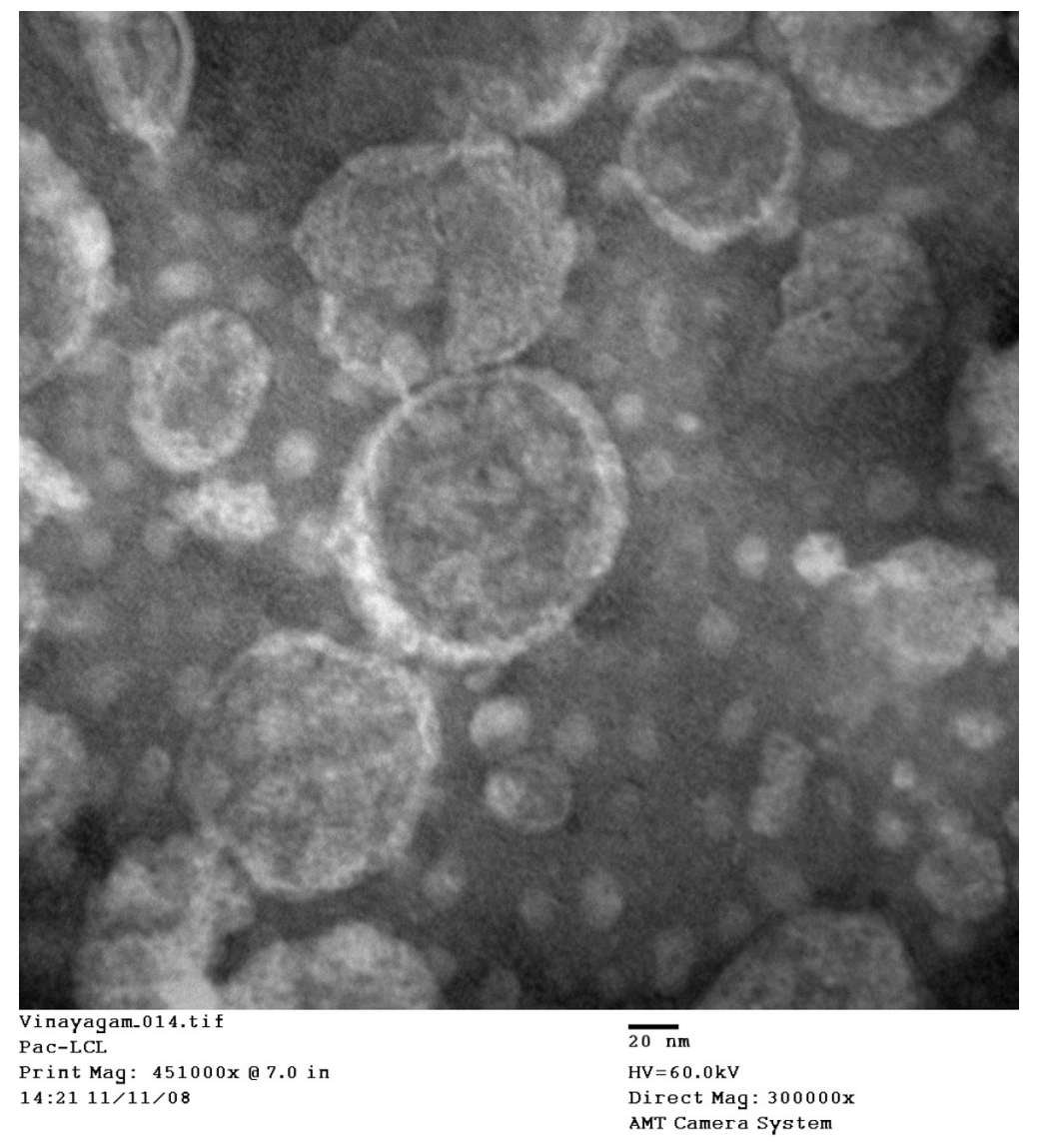

Figure 3-9. TEM image depicting morphology of Pac-LCL

Notes. The samples were prepared by negative staining with uranyl acetate using sequential two drop technique on formvar coated copper grids. The results are shown at a magnification of $300,000 x$. 

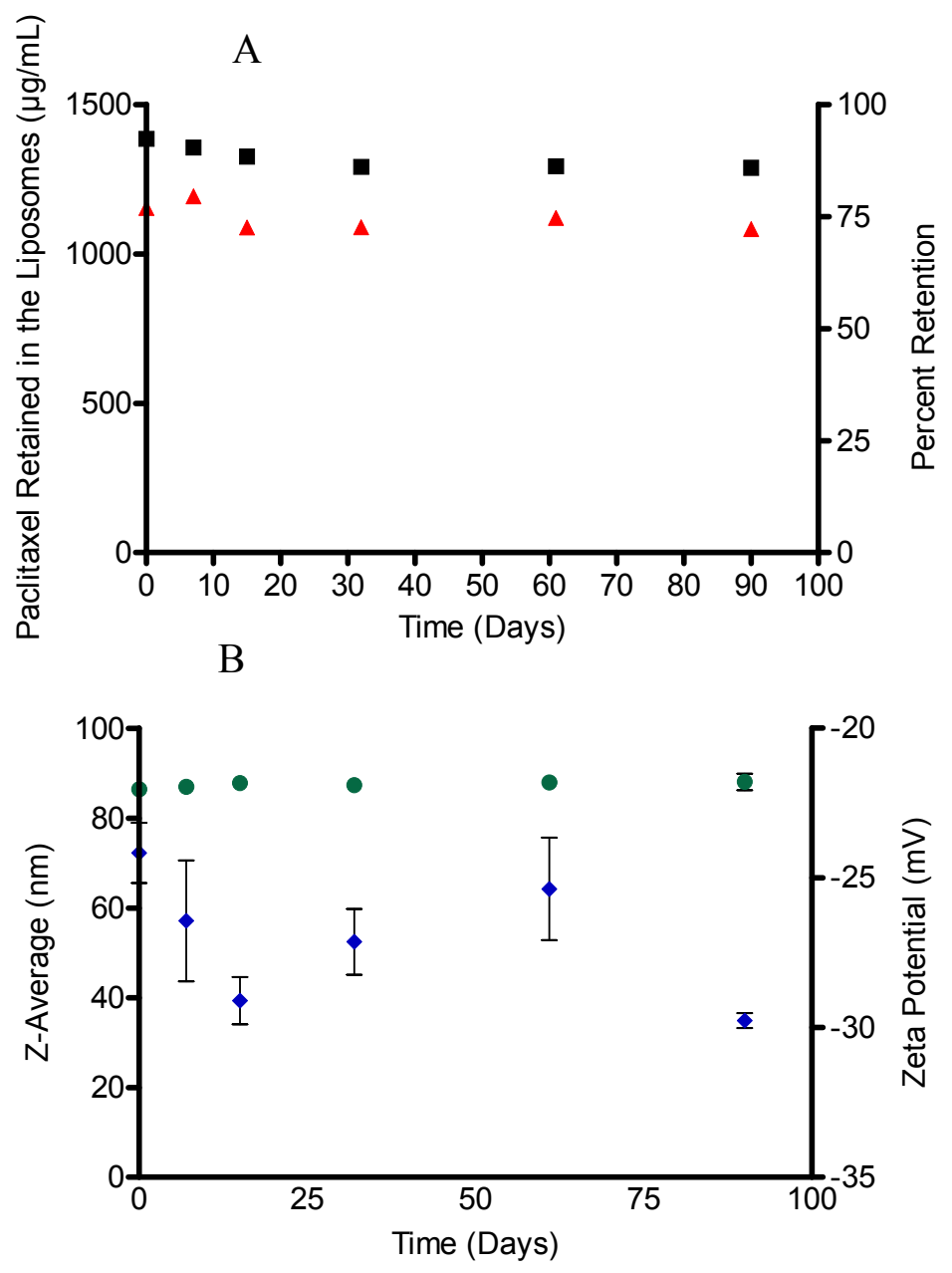

Figure 3-10. Stability of Pac-LCL dispersion at $5 \pm 3^{\circ} \mathrm{C}$

Notes. The liposome composition was HSPC: EPC: DSPE-PEG ${ }_{2000}$ : cholesterol (15: 75: 5: 5) with a total lipid concentration of $100 \mathrm{mM}$. The liposomes were stored at $5 \pm$ $3^{\circ} \mathrm{C}$ in $2 \mathrm{cc}$ amber vials. Samples were withdrawn at specific intervals. A. For the determination of paclitaxel retention, the samples were subjected to minicolumn centrifugation in Sephadex G-25 macro spin columns to separate free paclitaxel from total paclitaxel. The eluate containing entrapped paclitaxel was digested with ethanol, and quantified by RPHPLC method. The results indicate the mean \pm standard deviation for three injections ( $\Delta$ Paclitaxel concentration; - Percent paclitaxel retained in the liposomes). B. For PSD, and zeta potential, the results indicate mean \pm standard deviation of three independent measurements ( $\bullet$ PSD; $\diamond$ Zeta potential). 
allowed higher amounts of paclitaxel to be accommodated in the bilayer. Although high loading is achieved with these compositions, the entrapped paclitaxel precipitated out of the bilayer within few days of storage at $5 \pm 3^{\circ} \mathrm{C}$. The influence of drug-lipid ratio on drug loading showed that the loading increased with an increase in the drug-lipid ratio. But, LCL with a drug-lipid ratio of 1:30 was physically unstable. The short-term stability evaluation of liposomes prepared with different drug-lipid ratios demonstrated that 1:60 as the optimum drug-lipid ratio to achieve a loading of $1-1.3 \mathrm{mg} / \mathrm{mL}$ without the risk of physical instability. The optimized formulation was stable at $5 \pm 3^{\circ} \mathrm{C}$ for three months. The formulation and process parameters were optimized to achieve a physically stable formulation of Pac-LCL that maintains the size, charge, and integrity during storage. 


\section{CHAPTER 4. DEVELOPMENT OF PACLITAXEL-LOADED RGD CONJUGATED GAS-FILLED LIPOSOMES}

Cancer is a dangerous disease and is the second leading cause of death in United States $[2,3]$. About 1.5 million new cases of cancer and more than half-a-million deaths have been estimated in the United States for the year 2009 [4]. Solid tumors account for more than 85 percent of all the cases reported with cancer $[5,6]$. Breast cancer is the most commonly diagnosed form of solid tumors and is the second leading cause of death in western women. About 20-30\% women with breast cancers develop metastatic breast cancers. Chemotherapy is a form of systemic therapy of solid tumors and is also more useful in the treatment of metastatic cancers. The cytotoxic effect of these chemotherapeutic agents on cancer cells is highly beneficial for the therapy, but, adverse effects were also observed with these agents due to their effect on normal cells. Thus, there is a need for an effective drug delivery system that produces cytotoxic concentrations of the drug at the tumor sites without affecting normal tissues.

Paclitaxel is one of the most effective drugs used in chemotherapy of cancer and is a natural product isolated from bark of the Pacific Yew tree, Taxus brevifolia $[8,9]$. It exhibits cytotoxic activity by promoting the assembly of microtubules from tubulin dimers and stabilizes microtubules by preventing depolymerization. The stability results in inhibition of the normal dynamic reorganization of the microtubule network that is essential for vital interphase and mitotic cellular functions [9]. Paclitaxel is marketed as Taxol $^{\circledR}$ (Bristol-Myers Squibb Company, Princeton, NJ) for the treatment of ovarian, breast, non-small cell lung cancers, and AIDS related Kaposi's sarcoma [249]. Since paclitaxel is highly insoluble in water, Taxol ${ }^{\circledR}$ was formulated with ethanol and Cremophor ${ }^{\circledR}$ EL. Although the formulation is diluted before use, the concentration of Cremophor ${ }^{\mathbb{R}} \mathrm{EL}$ is high enough to cause serious hypersensitivity reactions on parenteral administration, which require pretreatment with antihistamines and corticosteroids [15]. In addition, the use of free paclitaxel can affect normal tissues and result in systemic toxicities such as myelosuppression, and peripheral neuropathy [17]. So, it is imperative to deliver paclitaxel locally to the tumor sites to minimize systemic toxicity. In addition, failure to maintain adequate concentration at the tumor microenvironment could result in regrowth of the tumor cells and development of resistance [5].

Liposomes are biocompatible carriers with ability to incorporate both lipophilic and hydrophilic drugs [23]. Encapsulating drugs within the vesicles has the potential advantage of higher drug carrying capacity compared to attaching them to single polymer chains [250]. Preparation of liposomes of paclitaxel can alter the pharmacokinetic and pharmacodynamic properties of the free drug and result in reduced toxicity and enhanced efficacy of the treatment [251]. In addition, flexibility in the design of liposomes allows tailoring to achieve passive as well as active targeting. The release of drug from these liposomes, at the target site, is determined by natural cytosolic mechanisms [23]. It will be an added advantage to have a control over drug release and/or uptake at the target site so that high cytotoxic concentrations can be achieved. Secondly, the unique nature of solid tumors with heterogeneity in vasculature, and high interstitial pressure pose a 
formidable barrier to drug delivery [19]. Apart from targeting and controlled release, it is also necessary to improve permeability at the tumor vasculature. Incorporation of gases in the bilayer of liposomes makes them acoustically active [205]. These echogenic liposomes have the potential for stress on activation by ultrasound, and eventually release the contents at the target site [35]. The disintegration of gas-filled species by contrast enhanced ultrasound produces liquid jets that can produce small gaps in the vasculature and improve the permeability [36]. Thus, cavitation effect of ultrasound on the echogenic liposomes provides a control over drug release, and also enhances drug permeability by producing small gaps in the endothelial walls of vasculature [36-39]. This phenomenon can be explored for targeted delivery wherein the targeted liposomes maintain basal levels of the drug by virtue of cytosolic transport, and by altering intensity of ultrasound, high cytotoxic concentrations of the drug can be achieved at the target site.

The size of these echogenic liposomes used for imaging is $\sim 800 \mathrm{~nm}$, and would be cleared rapidly by the reticuloendothelial system (RES). So, there is a need to control the size of these echogenic liposomes to approximately 150-200 nm to minimize clearance by the RES. An ideal carrier for therapeutic targeting to solid tumors must have a longer circulation time than those used for imaging and should possess targeting capability. In vivo circulation time can be increased by reducing the size of liposomes and/or modifying the surface with PEG derivatives [28]. Targeting capability can be achieved by attaching specific ligands onto the surface of the liposomes. The $\alpha_{v} \beta_{3}$ integrin receptors are highly expressed in actively proliferating endothelial cells of tumor vasculature and tumor cells [163, 235]. Peptides with Arg-Gly-Asp (RGD) motif in a cyclic framework have been shown to bind to these receptors and are effective in targeting solid tumors [172]. By attaching RGD peptide to the surface through PEG spacers, these liposomes can be targeted to solid tumors. The objective of this work is to develop parenteral echogenic RGD conjugated liposomes containing paclitaxel for ultrasound mediated targeted drug delivery. Owing to unique nature of the proposed parenteral dosage form, there is a potential to overcome the barriers in cancer chemotherapy.

\subsection{MATERIALS AND METHODS}

\subsubsection{Materials}

Paclitaxel was procured from 21CEC Pharmaceuticals (Bradenton, FL). The phospholipids used in this work were all of research grade. Egg phosphatidylcholine (EPC), hydrogenated soy phosphatidylcholine (HSPC) and 1,2-distearoyl-sn-glycero-3phoshoethanolamine-N-(poly(ethylene glycol)2000) (DSPE-PEG 2000 ) were obtained from Northern Lipids (Burnbay, BC, Canada). 1,2-distearoyl-sn-glycero-3phoshoethanolamine-N-(maleimide poly(ethylene glycol)2000) (maleimide DSPE$\mathrm{PEG}_{2000}$ ) was obtained from Avanti Polar Lipids (Alabaster, AL). Cholesterol, $\alpha-$ tocopherol, and sucrose were purchased from Sigma (St. Louis, MO). Mannitol was obtained from Mallinckrodt Chemicals (Phillipsburg, NJ). Cyclo (Arg-Gly-Asp-D-PheCys) peptide (cRGD) was obtained from Peptides International (Louisville, KY). 
Poloxamer 188 was obtained from BASF (Mount Olive, NJ). HPLC grade water, acetonitrile, and methanol were obtained from Fisher Scientific (Fair Lawn, NJ). HPLC grade chloroform was purchased from Acros Organics (Morris Plains, NJ). N-2hydroxyethylpiperazine-N'-2-ethanesulfonic acid (HEPES) buffer, 1M was obtained from Mediatech (Manassas, VA). All other chemicals and reagents were of analytical grade and used without further purification or characterization.

\subsubsection{Preparation of Paclitaxel-Loaded Long-Circulating Liposomes (Pac-LCL)}

The Pac-LCL was be prepared by the lipid film hydration-extrusion method using EPC, HSPC, DSPE-PEG 2000 , and cholesterol. The lipid film was prepared by dissolving paclitaxel, phospholipids, cholesterol, and $\alpha$-tocopherol in 9:1 chloroform-methanol. The solution obtained was evaporated in a round bottom flask using rotary evaporator under reduced pressure at $45^{\circ} \mathrm{C}$ for $4-6 \mathrm{~h}$. The dry film was hydrated with $50 \mathrm{mM}$ HEPES buffer $\mathrm{pH} 6.5$ at $65^{\circ} \mathrm{C}$ for $45-60 \mathrm{~min}$. The multilamellar vesicles (MLVs) thus obtained were sized by extrusion through stacked polycarbonate membrane filters of varying pore diameters under high pressure to obtain small unilamellar vesicles (SUVs). The SUVs were characterized for particle size distribution, zeta potential, and total and entrapped paclitaxel content.

\subsubsection{Preparation of Paclitaxel-Loaded cRGD Conjugated Targeted Long- Circulating Liposomes (Pac-RGD-TLCL)}

For the preparation of Pac-RGD-TLCL, a part of DSPE-PEG ${ }_{2000}$ was replaced with maleimide DSPE-PEG 2000 and the liposomes were prepared as described in section 4.1.2 to obtain SUVs with reactive maleimide functional groups. The maleimide-Pac-LCL thus obtained was conjugated with cRGD peptide by incubation at $25^{\circ} \mathrm{C}$ for $12 \mathrm{~h}$. Aliquots were withdrawn for the determination of conjugation efficiency. The unconjugated peptide remaining after the reaction was removed from the liposomes by equilibrium dialysis through 10,000 MWCO Slide-A-Lyzer dialysis cassettes (Thermo Fisher Scientific, Rockford, IL) against $50 \mathrm{mM}$ HEPES buffer $\mathrm{pH} 6.5$ at $5 \pm 3^{\circ} \mathrm{C}$ overnight. The conjugation efficiency was determined by quantifying free RGD in the liposomal dispersion by reversed phase high performance liquid chromatography (RPHPLC).

\subsubsection{Determination of Conjugation Efficiency}

Conjugation efficiency was determined by an indirect method to quantify the unconjugated cRGD peptide. The method was validated by adding different amounts of cRGD to the liposomes, and by quantifying unreacted peptide. An increasing amount of cRGD was added to a constant volume of maleimide-Pac-LCL. The same amounts were also added to Pac-LCL as a control for non-specific binding, and to HEPES buffer $\mathrm{pH}$ $6.5,50 \mathrm{mM}$ as a negative control. The reaction was performed in triplicates at three different amounts of cRGD. After the incubation period for the conjugation reaction, the 
samples were digested with absolute ethanol, and the free cRGD remaining was quantified by RPHPLC method. The gradient elution was performed using $0.05 \% \mathrm{v} / \mathrm{v}$ trifluoroacetic acid (TFA) in water (A) and $0.05 \% \mathrm{v} / \mathrm{v}$ TFA in acetonitrile (B) with a linear gradient of 10 to $60 \% \mathrm{~B}$ in $25 \mathrm{~min}$ at a flow rate of $1 \mathrm{~mL} / \mathrm{min}$. The amount of cRGD in HEPES buffer was taken as total unbound. The amount of cRGD consumed in Pac-LCL was taken as non-specific binding. The conjugation efficiency was calculated from free cRGD remaining in maleimide-Pac-LCL after subtracting the loss due to nonspecific binding. For day to day analysis, a slightly excess of cRGD peptide than required for the reaction was added to maleimide-Pac-LCL, Pac-LCL, and HEPES buffer. The conjugation efficiency was determined as described above.

\subsubsection{Preparation of Gas-Filled Pac-LCL (Pac-GFLCL) and Gas-Filled Pac- RGD-TLCL (Pac-RGD-GFTLCL)}

The Pac-LCL or Pac-RGD-TLCL was mixed with equal volume of sucrose solution containing poloxamer 188 with or without mannitol. The lyophilization cycle was designed based on the glass transition temperature of maximally freeze-concentrated amorphous phase $\left(\mathrm{Tg}^{\prime}\right)$, determined by differential scanning calorimetry (DSC). Lyophilization was carried out using a lab scale Virtis Genesis 25ES lyophilizer (The Virtis Company, Gardiner, NY). The primary drying temperature was selected based on $\mathrm{Tg}^{\prime}$ and the mixture was lyophilized in $2 \mathrm{cc}$ glass amber vials. The moisture content of the dry cake was monitored to ensure end point of secondary drying. On completion of the drying cycle, the vials were sealed under a partial vacuum. Perfluoropropane was filled in the headspace above the vials at atmospheric pressure to obtain Pac-GFLCL and Pac-RGD-GFTLCL. The vesicle size, zeta potential, total and entrapped paclitaxel content, and echogenicity were monitored in samples reconstituted with HPLC grade water. The vials were stored at $5 \pm 3^{\circ} \mathrm{C}$ until further use.

\subsubsection{Thermal Analysis by DSC}

The glass transition of maximally freeze concentrated amorphous phase $\left(\mathrm{Tg}^{\prime}\right)$ of mixture of liposomes and sucrose with or without mannitol was determined by differential scanning calorimetry (DSC) using a Q2000 differential scanning calorimeter equipped with refrigerated cooling system (TA Instruments, New Castle, DE). About 10$20 \mu \mathrm{L}$ of the liposome mixture prior to lyophilization was placed in T-Zero aluminum pans and hermetically sealed. An empty pan prepared in a similar manner was used as reference. The samples were equilibrated at $25^{\circ} \mathrm{C}$ and then cooled at $5^{\circ} \mathrm{C} / \mathrm{min}$ to $-70^{\circ} \mathrm{C}$. After equilibration, the samples were heated at the rate of $10^{\circ} \mathrm{C} / \mathrm{min}$ to $25^{\circ} \mathrm{C}$. 


\subsubsection{Characterization of Pac-LCL, Pac-RGD-TLCL, Pac-GFLCL, Pac-RGD- GFTLCL}

The average diameter (Z-avg) of the liposomes was determined using dynamic light scattering using Zetasizer Nano ZS (Malvern Instruments, Westborough, MA) equipped with $4.5 \mathrm{~mW}$ diode laser as a source of light operating at $670 \mathrm{~nm}$. Particle scattered photons were detected at an angle of $173^{\circ}$. The samples were suitably diluted with HPLC grade water for the determination of Z-avg. Three independent measurements were performed for each sample. Zeta potential was determined from electrophoretic mobility using samples suitably diluted with HPLC grade water.

The loading efficiency of paclitaxel in the liposomes was determined by minicolumn centrifugation method using Sephadex G-25 macro spin columns (Harvard apparatus, Holliston, MA) similar to the method described by Zhang et al [249] with modifications. Briefly, the Sephadex G-25 particles in the column were suspended in $0.1 \% \mathrm{w} / \mathrm{v}$ TFA and was allowed to swell for 15 minutes. The columns were centrifuged at $1500 \mathrm{~g}$ at $25^{\circ} \mathrm{C}$ for 4 min using IEC CL31R Multispeed Centrifuge (Thermo Scientific, Asheville, NC) to remove TFA. About $100 \mu \mathrm{L}$ of placebo LCL was added and centrifuged to saturate the column. The column saturation with placebo liposomes was repeated for a total of three times. The gel columns were placed in fresh collection tubes for use with drug-loaded liposomes. The Pac-LCL samples were diluted with $50 \mathrm{mM}$ HEPES buffer $\mathrm{pH}$ 6.5. About $100 \mu \mathrm{L}$ of the diluted sample was added to the gel column, and centrifuged for 4 minutes. The gas-filled liposomes were reconstituted with HPLC grade water prior to dilution. The eluate in the collection tube, containing only entrapped paclitaxel was analyzed by RPHPLC. Paclitaxel in the liposomes before passing through Sephadex G-25 was quantified for the total drug content. The HPLC system comprised of Waters 600 Controller, Waters 717 Plus Autosampler, and Waters 2996 Photodiode Array Detector (Waters Corporation, Milford, MA) was used. The RPHPLC analysis was performed using a Nova-Pak $\mathrm{C}_{18}$ column $(3.9$ x $150 \mathrm{~mm}, 4 \mu \mathrm{m})$ (Waters Corporation, Milford, MA). An isocratic elution was performed using acetonitrile-water in a ratio 55: $45 \mathrm{v} / \mathrm{v}$ at a flow rate of $1 \mathrm{~mL} / \mathrm{min}$, and detection wavelength of $230 \mathrm{~nm}$. The liposomes were digested with absolute ethanol, and suitably diluted before injection. The encapsulation efficiency was calculated using the formula, Entrapment efficiency $=($ Entrapped paclitaxel $\mathrm{x}$ 100)/Total paclitaxel).

The moisture content of the lyophilized cake was determined using an Orion AF8 Volumetric Karl Fischer Titrator (Orion Research Inc., Boston, MA). The titrator was calibrated on each day of analysis with a known quantity of water. About $75 \mathrm{mg}$ of the dried sample was weighed accurately and added to the titration vessel. The titration was started after allowing one minute for extraction.

Morphology of the liposomes was characterized by transmission electron microscopy (TEM) using a negative staining technique. Sequential two droplet method was used for staining liposome samples with $2 \% \mathrm{w} / \mathrm{v}$ uranyl acetate on 400 -mesh formvar support film on copper specimen grid (Electron Microscopy Sciences, Hartfield, PA). The TEM 
images were acquired using JEOL 2000EX transmission electron microscope (JEOL USA, Inc., Peabody, MA) equipped with high resolution digital camera.

\subsubsection{Determination of Echogenicity}

Acoustic activity of Pac-GFLCL and Pac-RGD-GFTLCL was characterized using an in vitro method utilizing Phillips ATL HDI-5000 ultrasound system and broadband L12-5 transducer (Philips Healthcare, Andover, MA). A rectangular plexiglass chamber was filled with $15 \mathrm{~mL}$ of normal saline (NS). A small magnetic stir bar was placed in the chamber to enable uniform mixing. The chamber was then placed on a magnetic stirrer. The transducer was positioned such that it has direct contact with NS filled in the tank. The images were acquired with a mechanical index setting of 0.1. The Pac-GFLCL was reconstituted with HPLC grade water. After acquiring the background image for $5 \mathrm{sec}$., $50 \mu \mathrm{L}$ of the reconstituted liposomal product was injected into the tank with a $1 \mathrm{cc}$ syringe, and the images were acquired for one minute. All image sequences were analyzed offline using a custom program using MATLAB 7.0.4 software (The MathWorks, Inc., Natick, MA). The pixel values in the region of interest (ROI) were compared to the pixel values in the background. The number of folds increase in mean gray scale value (MGSV) in the ROI over the background was calculated as the measure of acoustic activity. For demonstrating the ability to disintegrate the Pac-RGD-GFTLCL, VisualSonics ${ }^{\mathrm{TM}}$ Vevo $^{\circledR} 2100$ ultrasound instrument with MS 200 transducer (Visualsonics, Inc., Toronto, Ontario, Canada) was used in contrast mode. A MTV-1 restrained tail vein infusion catheter (Braintree Scientific, Braintree, MA) was mounted in the plexiglass tank such that a portion of the catheter is completely immersed in water filled in the tank (Figure 4-1). The MS 200 transducer was positioned above the catheter with water in the tank as interface. The gas-filled liposomes were reconstituted with HPLC water, the sample was drawn into a $1 \mathrm{cc}$ syringe, and the syringe was connected to the catheter. The sample was slowly injected through the catheter and the liposomes were visualized by ultrasound at $12.5 \mathrm{MHz}$ center frequency at $10 \%$ power. The flow was stopped during the measurement sequence to determine the ability to disintegrate gasfilled liposomes with ultrasound. A high intensity pulse was applied for a duration of one second at $50 \%$ of the cine loop sequence. The contrast mean power at the region of interest was calculated.

\subsubsection{In vitro Drug Leakage Study}

Paclitaxel leakage from Pac-RGD-GFTLCL was determined by equilibrium dialysis of the liposomal dispersion using 10,000 MWCO Slide-A-Lyzer dialysis cassettes (Thermo Fisher Scientific, Rockford, IL) against pH 7.4 phosphate buffered saline (PBS) containing $0.1 \% \mathrm{w} / \mathrm{v}$ Tween 80 in a jacketed beaker maintained at $37^{\circ} \mathrm{C}$. Samples of 1 $\mathrm{mL}$ were withdrawn at specific intervals for a period of 72 hours, and then $1 \mathrm{~mL}$ of fresh medium was added to the release vessel for replacement. The samples were analyzed by RPHPLC method. The drug released/leaked was evaluated as a function of time. 


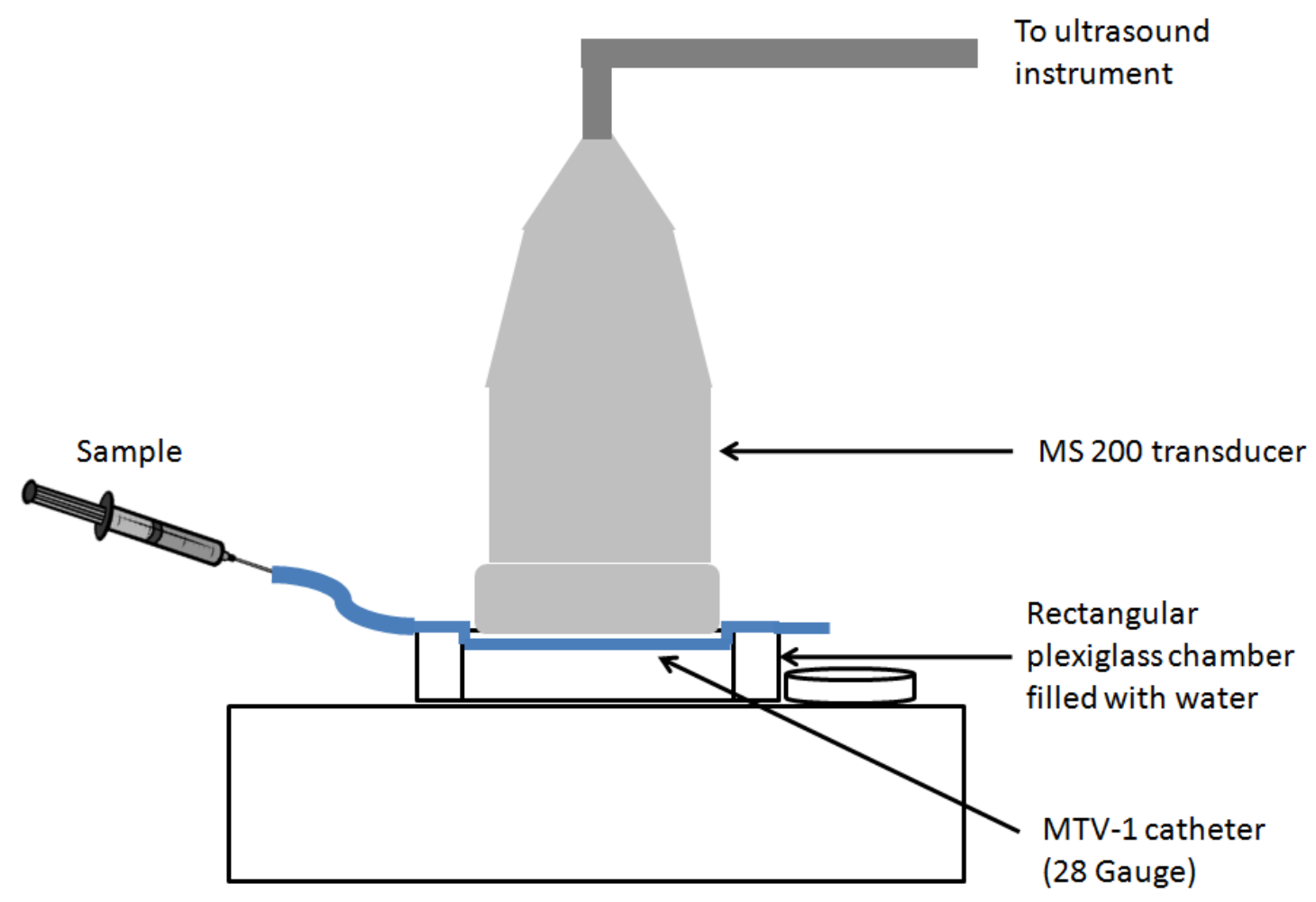

Figure 4-1. The in vitro model used for demonstrating disintegration of gas-filled liposomes

Notes. The apparatus used for demonstrating disintegration of gas-filled liposomes consists of a rectangular plexiglass chamber filled with water. A tail vein infusion catheter was mounted so that it is immersed in water. The MS 200 transducer was placed above the catheter using water as the interface. The reconstituted echogenic liposomes were injected through the catheter, viewed and disintegrated by ultrasound. The diagram is not drawn to scale. 


\subsubsection{Stability Evaluation of Pac-RGD-GFTLCL}

Stability of Pac-RGD-GFTLCL was evaluated for three months. The optimized formulation in $2 \mathrm{cc}$ amber glass vials was stored at $5 \pm 3^{\circ} \mathrm{C}$ for three months. Samples were withdrawn at specific intervals and analyzed for total and entrapped paclitaxel, entrapment efficiency, PSD, zeta potential, and moisture content.

\subsection{RESULTS AND DISCUSSION}

The optimized composition of paclitaxel-loaded liposomes from chapter 3 was mixed with mannitol solutions containing $0.3 \% \mathrm{w} / \mathrm{v}$ poloxamer 188 and different amounts of sucrose, and then lyophilized. The particle size of the liposomes increased to above 400 $\mathrm{nm}$ irrespective of the concentration of sucrose in the formulation. This could be due to fusion or aggregation of liposomes during lyophilization. The results were in contrast to the optimized formulation obtained for the prototype formulation in chapter 2 . The possible reasons for the increase in PSD could be the changes in the lipid composition, lipid concentration, and inclusion of paclitaxel in the formulation. So, attempts were made to lyophilize the formulation with sucrose and poloxamer 188 to determine the optimum lipid-sugar ratio for a better control in PSD. Then, mannitol was included in the composition to determine its effects on PSD and echogenicity. The optimization of the formulation for lyophilization was performed with Pac-LCL, and then Pac-RGD-TLCL was used with the optimum composition to prepare Pac-RGD-GFTLCL. Comparisons were made between Pac-GFLCL, and Pac-GFTLCL.

\subsubsection{Effect of Lyoprotectant and the Manufacturing Process on PSD of Pac- GFLCL}

Phospholipids can undergo hydrolysis or oxidation during storage of liposomes in the form of dispersion. As a result, the liposomes can aggregate or fuse together resulting in an increase in particle size distribution. Freeze drying or lyophilization can prevent hydrolysis of phospholipids and physical degradation during storage of the liposomes [34]. However, lyophilization process itself can cause loss of encapsulated drug and fusion of the vesicles. Interaction of water with hydrophilic phosphate head groups of phospholipids plays a key role in the formation of liposomes and hence, removal of water presents more challenges. Inclusion of lyoprotectants can help maintain the structure, and integrity of liposomes during lyophilization [33]. The effect of different lipid-sugar ratios on PSD of Pac-LCL after lyophilization was evaluated with sucrose. The heating profile of DSC showed that the $\mathrm{Tg}^{\prime}$ of these liposome-sucrose mixtures was between - 35 to $39^{\circ} \mathrm{C}$ (Figure 4-2). The trend showed that there was a slight increase in the $\mathrm{Tg}^{\prime}$ with an increase in the concentration of sucrose. Figure 4-3 shows the effect of lipid-sugar ratio on PSD of the liposomes after lyophilization. The increase in size of liposomes after lyophilization was minimized at lipid-sugar ratios below 1:5. Further changes in the ratio showed a trend of decrease in size of the liposomes with increasing amounts of sucrose in the formulation up to a ratio of 1:7. Additional increases in sucrose concentration did not 


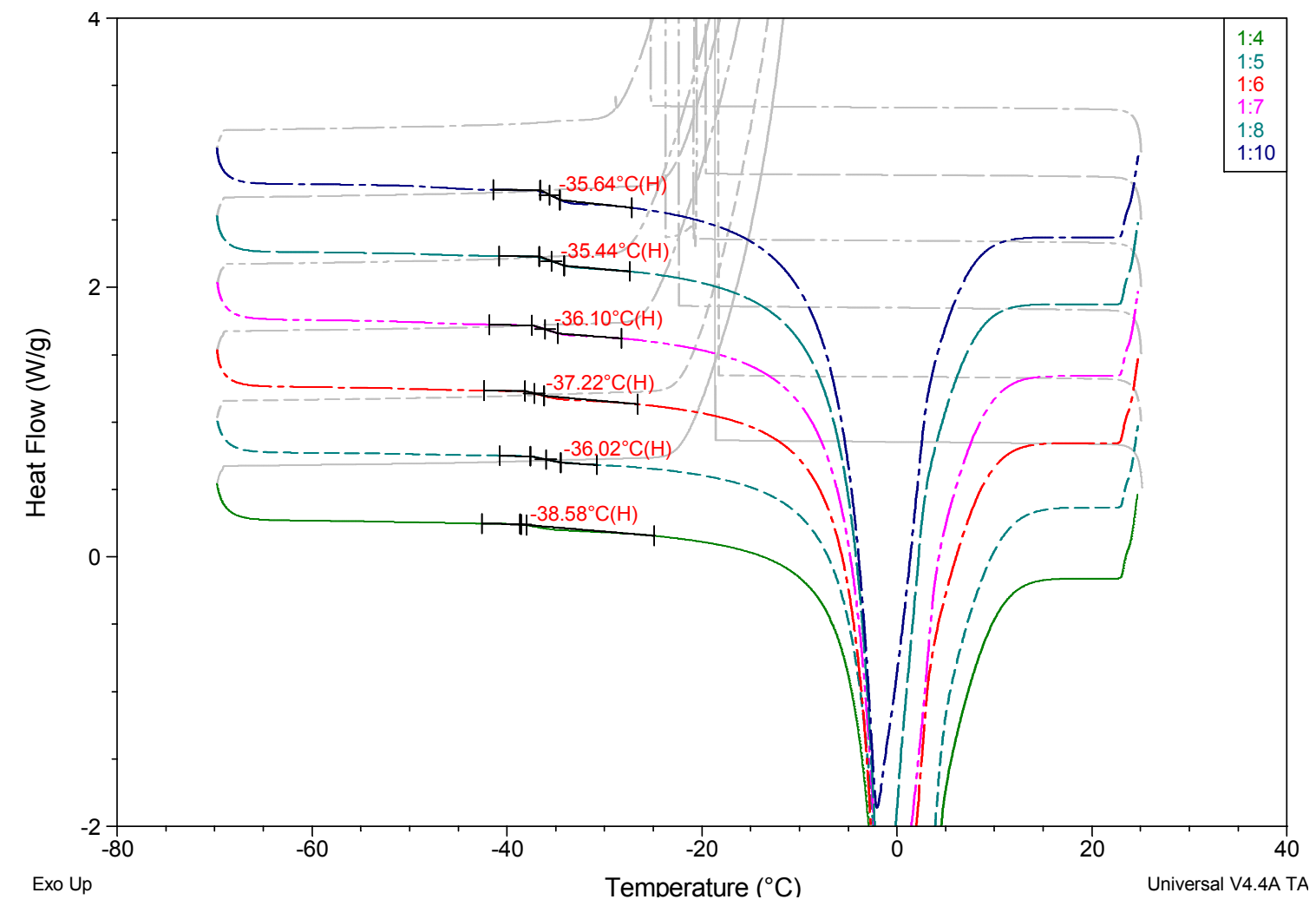

Figure 4-2. DSC thermograms showing the effect of lipid-sucrose ratio on $\mathrm{Tg}^{\prime}$

Notes. The liposomes were mixed with equal volume of sucrose solution containing $0.3 \% \mathrm{w} / \mathrm{v}$ poloxamer 188 at different lipid-sucrose ratios. The samples were hermetically sealed in aluminum pans, and cooled at the rate of $5^{\circ} \mathrm{C} / \mathrm{min}$ to $-70^{\circ} \mathrm{C}$. After equilibration at $-70^{\circ} \mathrm{C}$, the samples were heated at the rate of $10^{\circ} \mathrm{C} / \mathrm{min}$ to $25^{\circ} \mathrm{C}$. The heating profile is shown in the figure. Heat flow is shown on an arbitrary scale. 


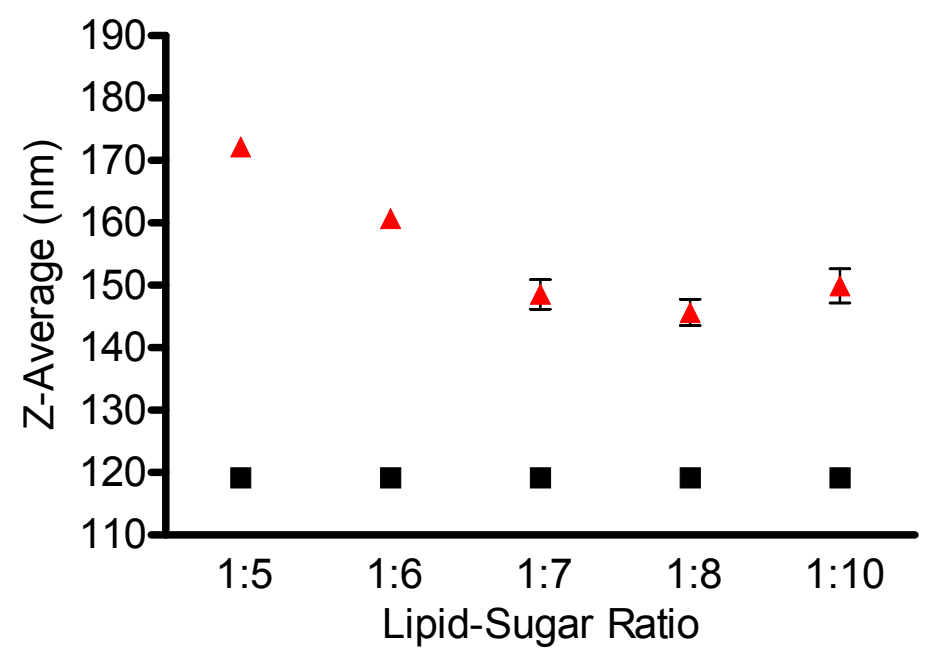

Figure 4-3. Effect of lipid-sugar ratio on PSD after lyophilization of Pac-LCL

Notes. The liposomes were prepared by lipid film hydration extrusion method. The PSD was determined before and after freeze drying in presence of varying lipid-sugar ratios with sucrose. The freeze dried paclitaxel-loaded gas-filled liposome samples were reconstituted with HPLC grade water for the measurement of PSD. The results indicate mean \pm standard deviation of three independent measurements ( $\square$ Before lyophilization; $\Delta$ After lyophilization). 
show any appreciable effect. The results were consistent with findings in literature on the ability of different saccharides to act as cryoprotectants for small unilamellar vesicles $[220,221]$. The ability of these saccharides to replace water molecules of hydration by forming hydrogen bond with the polar phospholipid group, at same binding site as water, was suggested as the mechanism for cryoprotection. These reports also suggested that the fraction of liposomes unaffected during dehydration was dependant on the sugar to lipid molar ratio. The lipid-sugar ratio of 1:7 was selected as optimum for further studies. In addition, the lyoprotectants are known to provide better protection to liposomes during lyophilization when they are present on both sides of the membrane [33]. The total amount of sugar was divided into internal-external ratio and the internal sucrose portion was added to the hydration medium during the preparation of liposomes. The external sucrose along with poloxamer 188 was mixed with preformed Pac-LCL containing different internal amounts of sucrose. The effect of internal-external ratio of sucrose on PSD of Pac-LCL before and after lyophilization is presented in Figure 4-4. The liposomes prepared with internal sucrose showed minimal increase in size compared to those prepared without internal sucrose. This could be attributed to the lyoprotectant effect on inner portions of the bilayer. Further increase in concentration of internal sucrose did not show any effect as reported in literature [226] suggesting only a small fraction of lyoprotectant is required inside the aqueous core of the liposomes. Although these gas-filled liposomes were of size $150-200 \mathrm{~nm}$ in size, within the target size required for drug delivery, a closer observation of the intensity distribution of the average diameter showed a secondary peak appearing at approximately $1 \mu \mathrm{m}$ diameter suggesting the process needs to be fine tuned further for a narrow size distribution.

The effect of number of extrusion cycles used in the preparation of Pac-LCL on the PSD after lyophilization was evaluated (Figure 4-5). The vesicle size decreased with increase in number of extrusion cycles. It was interesting to note that the size after lyophilization was almost similar for all the liposomes prepared with different numbers of extrusion cycles even though they were of different size before lyophilization. The difference between the size before and after lyophilization increased with increasing numbers of extrusion cycles. The polydispersity index (PDI), which describes variation in the size distribution, showed a trend of increase suggesting that the distribution was broader when the number of extrusion cycles was increased. Folds increase in PDI increased with increase in the number of extrusion cycles though not significant. One extrusion cycle with minimal increase in size and PDI after lyophilization was selected for further evaluation. Further attempts were made to minimize the increase in PDI after lyophilization. The effect of HEPES buffer, concentration of the buffer, rate of cooling during the freezing phase of lyophilization process, and primary drying temperature on PSD after lyophilization of Pac-LCL was evaluated. When the primary drying temperature was set closer to the $\mathrm{Tg}^{\prime}$ of the frozen liposome-sucrose mixture, the increase in PDI after lyophilization was minimized (Figure 4-6). 


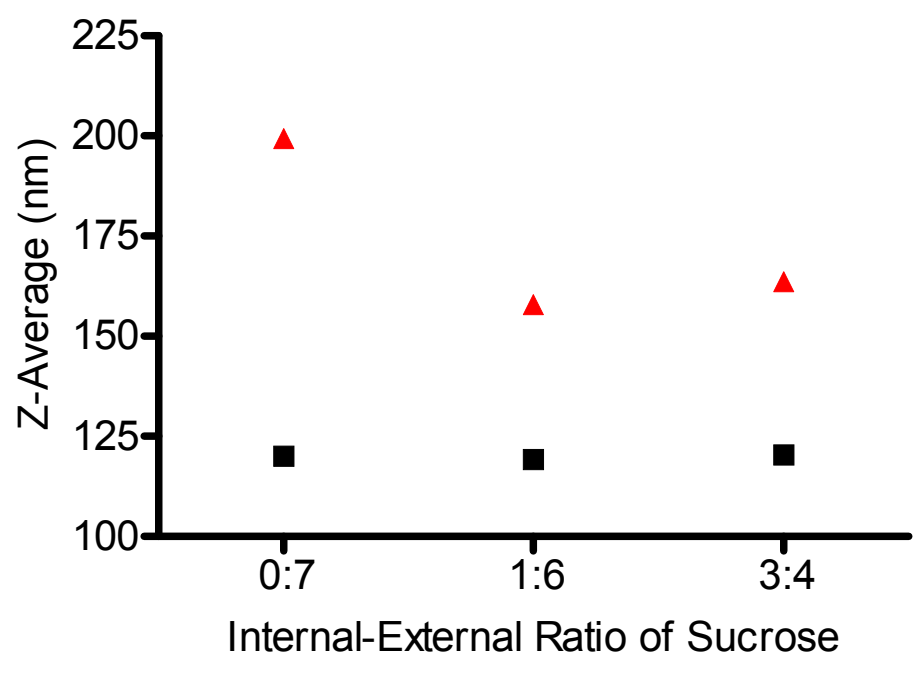

Figure 4-4. Effect of inravesicular-extravesicular ratio of sucrose on PSD of Pac-LCL

Notes. The liposomes were prepared by lipid film hydration extrusion method with different amounts of intravesicular sucrose. The PSD was determined before and after freeze drying in presence of varying extravesicular amounts of sucrose. The freeze dried paclitaxel-loaded gas-filled liposome samples were reconstituted with HPLC grade water for the measurement of PSD. The results indicate mean \pm standard deviation of three independent measurements ( $\boldsymbol{\|}$ Before lyophilization; $\Delta$ After lyophilization). 


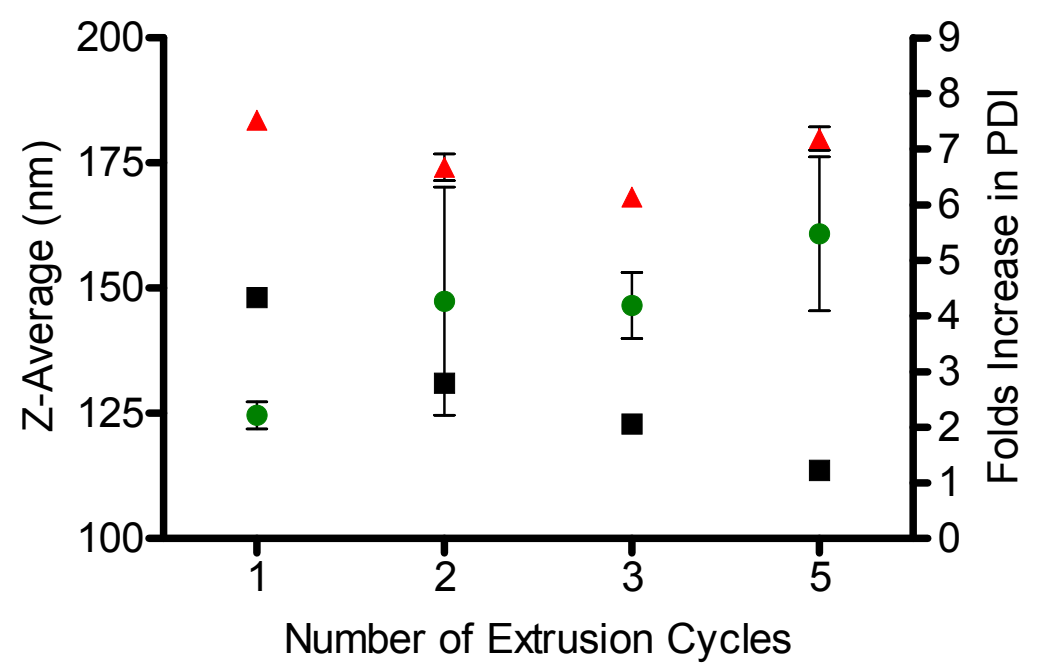

Figure 4-5. Effect of number of extrusion cycles on the PSD and PDI after lyophilization

Notes. The liposomes were prepared by lipid film hydration extrusion method with different numbers of extrusion cycles. The PSD was determined before and after freeze drying. The freeze dried paclitaxel-loaded gas-filled liposome samples were reconstituted with HPLC grade water for the measurement of PSD and PDI. The results indicate mean \pm standard deviation of three independent measurements ( Particle size before lyophilization; $\Delta$ Particle size after lyophilization; • Folds increase in PDI after lyophilization). 


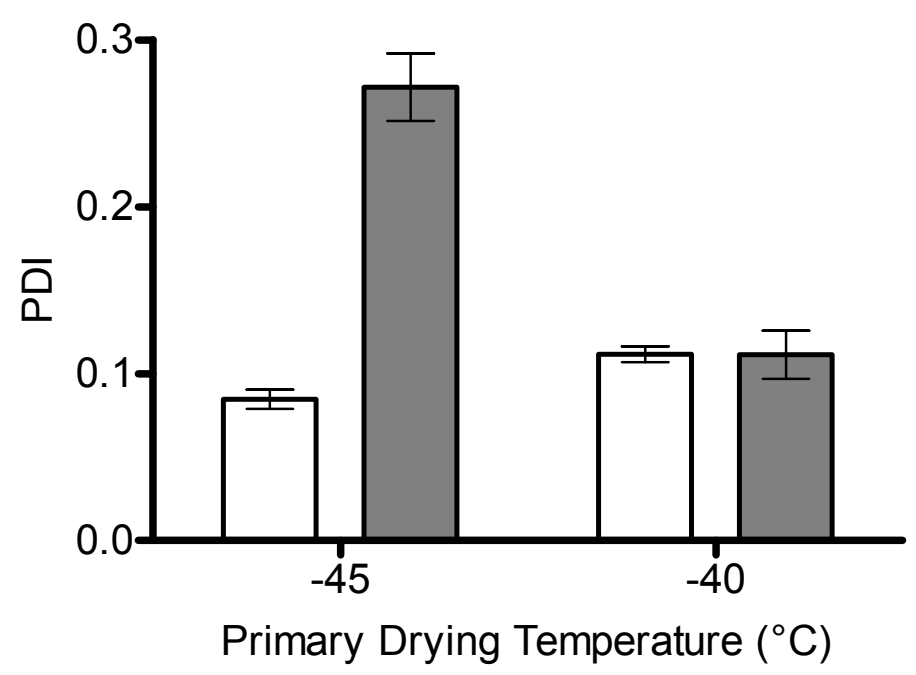

Figure 4-6. Effect of primary drying temperature on PDI after lyophilization of PacLCL

Notes. The liposomes were prepared by lipid film hydration extrusion method. The primary drying of the frozen liposome-sucrose mixture was started at $-45^{\circ} \mathrm{C}$ or $-40^{\circ} \mathrm{C}$. The PSD was determined before and after freeze drying. The freeze dried paclitaxel-loaded gas-filled liposome samples were reconstituted with HPLC grade water for the measurement of PSD and PDI. The results indicate mean \pm standard deviation of three independent measurements ( $\square$ Before lyophilization; $\square$ After lyophilization). 


\subsubsection{Effect of Mannitol Concentration}

Mannitol undergoes crystallization from frozen aqueous solutions during the warming phase of freeze drying [237-239]. Crystallization of mannitol has been shown to play a critical role in the preparation of echogenic liposomes [206]. The crystallization causes rupture in the bilayer of liposomes due to which air/gas is entrapped in the liposomes. Mannitol was included in the formulation to evaluate its effect on the PSD and echogenicity of paclitaxel-loaded gas-filled liposomes. DSC thermograms showed that there was a decrease in $\mathrm{Tg}^{\prime}$ with an increase in mannitol concentration in liposomesucrose mixtures containing poloxamer 188 (Figure 4-7). However, no exothermic peak, characteristic of crystallization of mannitol was observed at the concentrations studied. This could be due to the presence of high concentrations of non-crystallizing solute sucrose in the formulation which minimized the crystallization of mannitol during the heating phase. The effect of mannitol on the PSD of Pac-LCL before and after lyophilization is presented in Figure 4-8. An increase in mannitol concentration resulted in an increase in average diameter of Pac-GFTLCL. Although there was no evidence of crystallization of mannitol from the DSC thermograms, inclusion of mannitol played a role in determining the particle size after lyophilization. The particle size distribution was broader in presence of mannitol as exemplified by an increase in PDI. The examination of intensity distribution for PSD revealed that formulations containing mannitol have a bimodal distribution with secondary peak appearing at size greater than $1 \mu \mathrm{m}$. In contrast, a single unimodal distribution was observed in the absence of mannitol. The possible reasons for the effect of mannitol on the PSD could be due to the crystallization of mannitol which might have occurred at a level that is not perceivable by DSC thermograms at the concentrations studied. The effect of crystallization of mannitol during warming phase of lyophilization of liposomes is well known. It ruptures the bilayer and thereby increases the entrapment of gas in the bilayer resulting in an increase in size. Another possibility is that a decrease in the concentration of lyoprotectant sucrose in the formulation which was adjusted to accommodate mannitol to maintain a constant lipid-sugar ratio. Adequate concentrations of sucrose was not available to provide lyoprotectant effect which could have resulted in rupture to the bilayer, increased entrapment of gas in the bilayer, and an increase in size.

Ultrasound measurements showed that the formulations containing mannitol were more echogenic than the formulations without mannitol (Figure 4-9). The formulations prepared with different concentrations of mannitol were comparable with $\sim 50$-fold increase in reflectivity over background. In the absence of mannitol, $\sim 20$ fold increase in reflectivity was observed. The echogenicity of the formulation that contained only sucrose was similar to that observed with prototype formulation described in Chapter 2 (Figure 2-7) though only a fourth of the concentration was used. This could be attributed to changes in the lipid concentration, lipid composition, and inclusion of a hydrophobic drug paclitaxel in the bilayer of the liposomes. Even though the presence of mannitol contributes to improve acoustic activity of Pac-GFLCL similar to the formulations described in Chapter 2, its presence has a negative impact on PSD and PDI. Mannitol containing formulations were slightly larger in size with a broader PDI compared to the formulation without mannitol. Though the echogenicity was lower with the formulation 


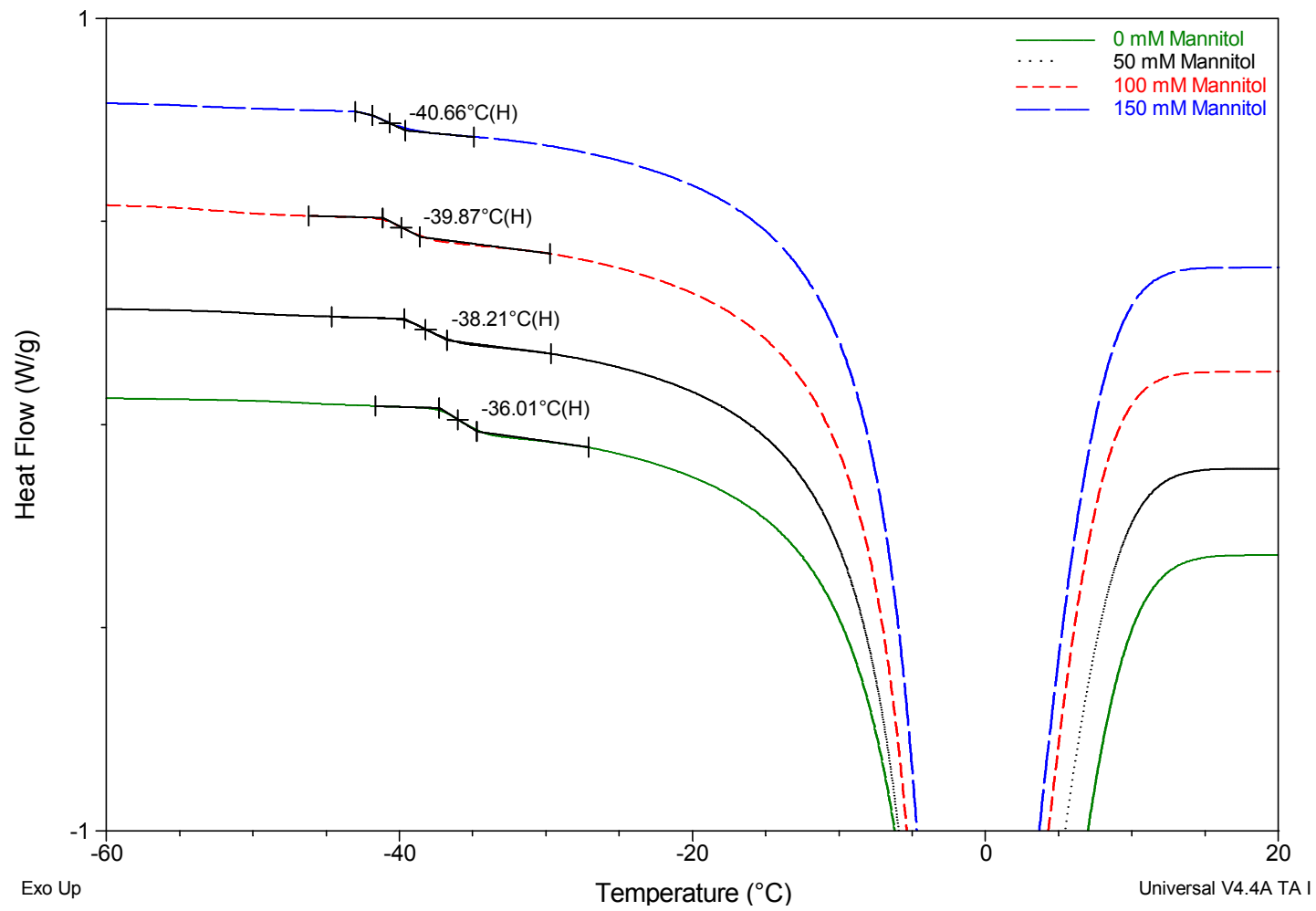

Figure 4-7. Effect of mannitol concentration on $\mathrm{Tg}^{\prime}$

Notes. The liposomes were mixed with equal volume of sucrose solution containing $0.3 \% \mathrm{w} / \mathrm{v}$ poloxamer 188 and different concentrations of mannitol. The samples were hermetically sealed in aluminum pans, and cooled at the rate of $5^{\circ} \mathrm{C} / \mathrm{min}$ to $-70^{\circ} \mathrm{C}$. After equilibration at $-70^{\circ} \mathrm{C}$, the samples were heated at the rate of $10^{\circ} \mathrm{C} / \mathrm{min}$ to $25^{\circ} \mathrm{C}$. A segment of heating profile is shown in the figure. The heat flow is shown on an arbitrary scale. 


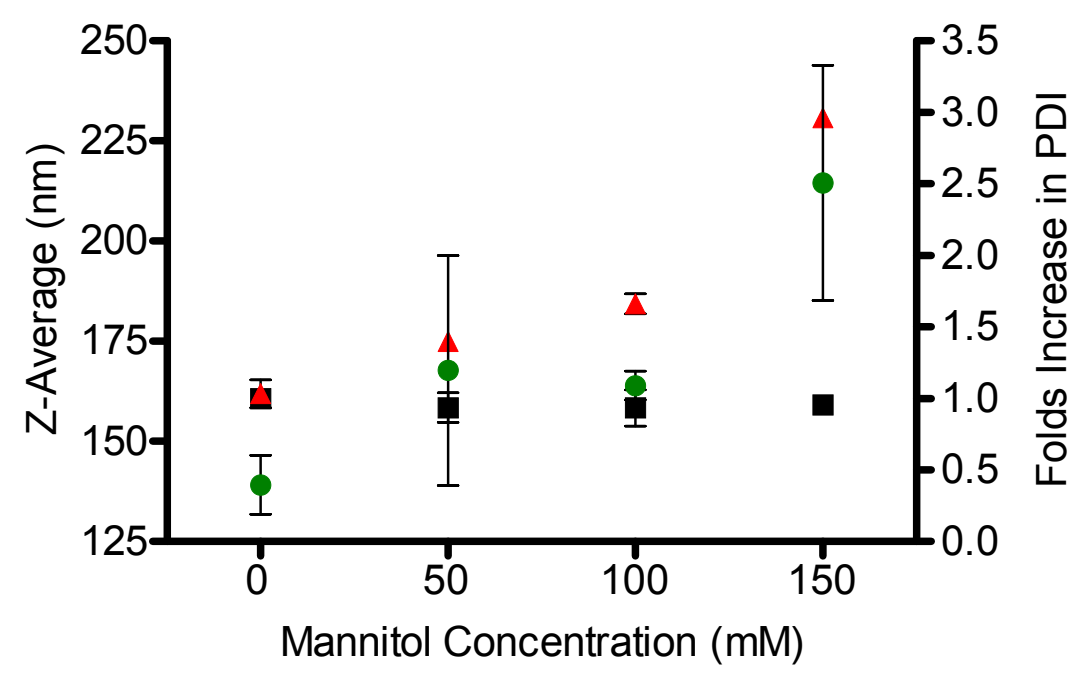

Figure 4-8. Effect of mannitol concentration on the PSD and PDI after lyophilization

Notes. The liposomes were prepared by lipid film hydration extrusion method. The Pac-LCL was mixed with equal volume of sucrose solution containing $0.3 \% \mathrm{w} / \mathrm{v}$ poloxamer 188 at different concentrations of mannitol. The PSD was determined before and after freeze drying. The freeze dried paclitaxel-loaded gas-filled liposome samples were reconstituted with HPLC grade water for the measurement of PSD and PDI. The results indicate mean \pm standard deviation of three independent measurements ( Particle size before lyophilization; $\Delta$ Particle size after lyophilization; • Folds increase in PDI after lyophilization). 


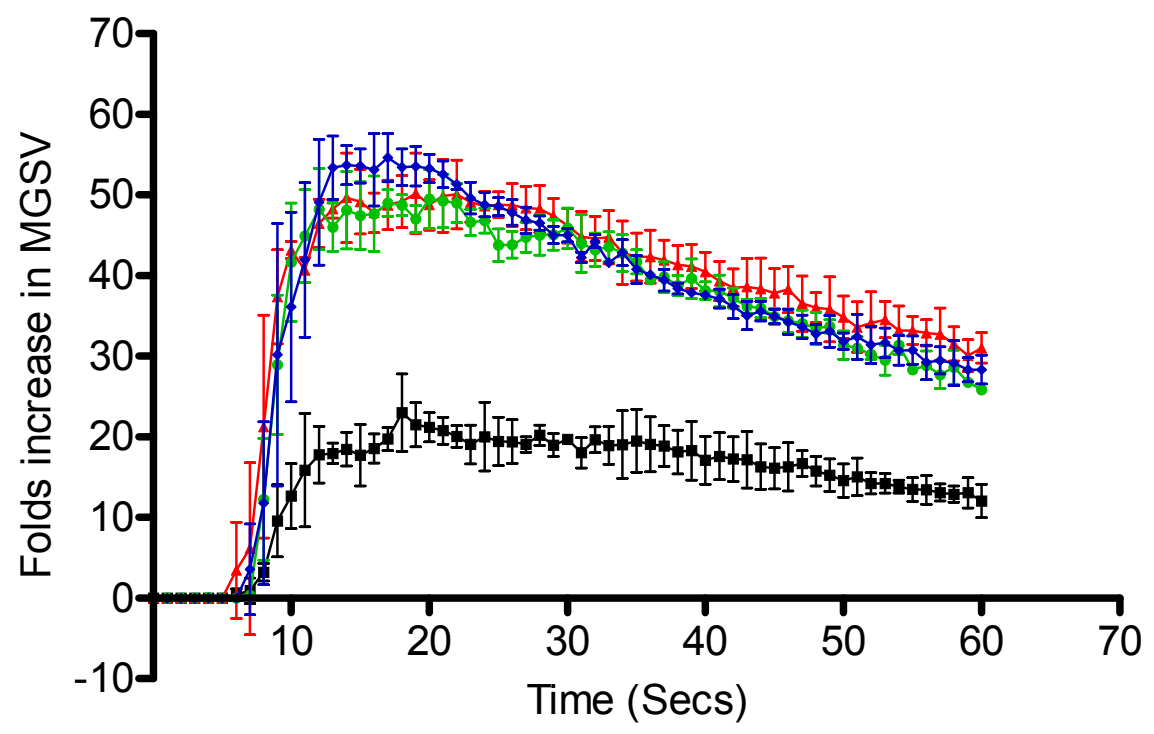

Figure 4-9. Effect of mannitol concentration on echogenicity of Pac-GFLCL

Notes. The echogenicity was determined in an in vitro model with Philips ATL-HDI 5000 ultrasound system and L12-5 broadband transducer. The Pac-GFLCL samples were reconstituted with HPLC grade water for the measurement of echogenicity. After acquiring background images for $5 \mathrm{sec} ., 50 \mu \mathrm{L}$ of the sample was added using a syringe, and the acquisition was continued for up to one minute. The data was processed off-line with a custom MATLAB program. Three ROIs were randomly selected, and the average increase in mean grayscale intensity in the ROI from the background was calculated. The results represent mean \pm standard deviation for three samples (Mannitol concentrations were $0 \mathrm{mM}$; $\Delta 50 \mathrm{mM}$; $100 \mathrm{mM}$; $150 \mathrm{mM}$ ). 
prepared without mannitol, if the acoustic activity is adequate for visualization and disintegration, this formulation is expected to have a longer in vivo circulation times due to smaller size. On the other hand, if the acoustic activity is not adequate for visualization and disintegration, the mannitol containing formulations can be taken forward with a slight compromise in their circulation times due to slightly larger size than sucrose containing formulations. Although mannitol containing formulations have the potential for further development, we took the formulation without mannitol for further studies to have better control over the PSD.

\subsubsection{In vitro Leakage Study with Pac-GFTLCL}

The release or leakage profile of the reconstituted Pac-GFTLCL is presented in Figure 4-10. An approximately $7 \%$ of the encapsulated paclitaxel was released or leaked from the Pac-GFTLCL formulation over $72 \mathrm{~h}$ period in physiological conditions. These results indicate that the amount of free paclitaxel available in the systemic circulation can be minimized by loading paclitaxel in the Pac-GFTLCL and therefore, undesirable toxicities due to systemic exposure of paclitaxel to normal tissues can be minimized.

\subsubsection{Conjugation Efficiency of cRGD to Maleimide-Pac-LCL}

One of the most stable and most efficient conjugation reactions in chemistry, the formation of covalent thioether bond by the reaction between sulfhydryl group and maleimide group [172, 242-244] was used for conjugation of cRGD to maleimide DSPE$\mathrm{PEG}_{2000}$ present on the surface of preformed liposomes. The method used to determine the conjugation efficiency was validated by adding different amounts of cRGD to the reaction buffer, Pac-LCL, and maleimide-Pac-LCL. The amount of unreacted cRGD was quantified by the RPHPLC method from the samples digested with absolute ethanol. The cRGD recovered from the reaction buffer was taken as total unbound. The cRGD consumed by the Pac-LCL which does not contain the reactive maleimide functional group was taken as non-specific binding. The amount of cRGD consumed by maleimidePac-LCL after subtracting for non-specific binding was taken as the amount conjugated to the liposomes. Figure 4-11 shows the amount of cRGD recovered from buffer, PacLCL, and maleimide-Pac-LCL. Almost all of the cRGD added to the reaction buffer was recovered after the incubation period. There was a decrease in the amount of cRGD recovered from Pac-LCL. This could be attributed to non-specific charge interactions of cRGD containing residual positive charge with negatively charged liposomes. The nonspecific binding was constant and did not change with an increase in the amount of cRGD added to the liposomes suggesting that it can be saturated. Almost all the cRGD was consumed in the reaction with maleimide-Pac-LCL when the amount of peptide added was equivalent to that is needed for the reaction. The amount consumed in the reaction slightly increased with increasing amounts of cRGD added suggesting the conjugation efficiency can be increased by adding excess of cRGD peptide. The unreacted peptide can be removed after the end of the reaction by equilibrium dialysis or ultrafiltration. Further reactions for conjugation were performed at slightly excess of 


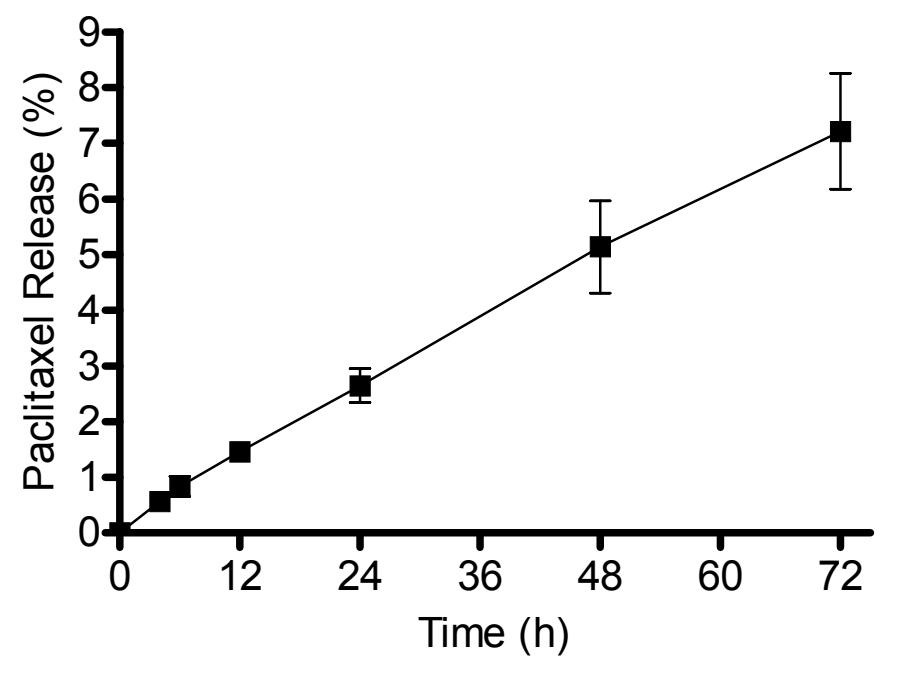

Figure 4-10. In vitro drug leakage study of Pac-GFLCL

Notes. Pac-GFTLCL was reconstituted with HPLC grade water and $3 \mathrm{~mL}$ of the reconstituted sample was dialyzed through 10,000 MWCO Slide-A-Lyzer dialysis cassettes against $\mathrm{pH} 7.4$ phosphate buffered saline (PBS) containing $0.1 \% \mathrm{w} / \mathrm{v}$ Tween 80 in a jacketed beaker maintained at $37^{\circ} \mathrm{C}$. Samples of $1 \mathrm{~mL}$ were withdrawn at specific intervals for a period 72 hours and analyzed by RPHPLC method. The results indicate mean \pm standard deviation of results from three dialysis cassettes. 


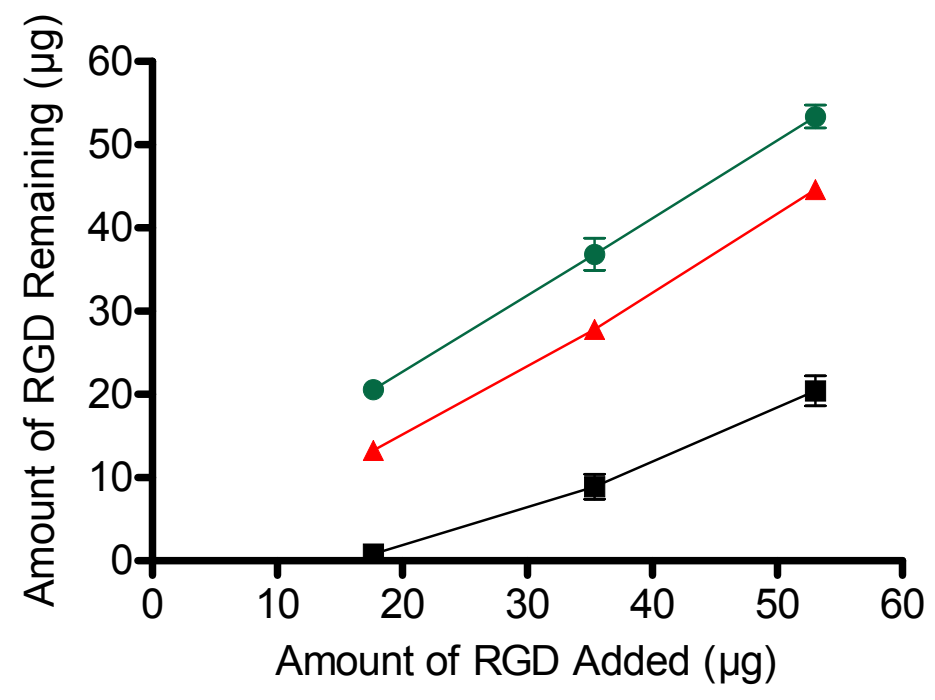

Figure 4-11. Verification of conjugation efficiency of cRGD

Notes. An increasing amount of cRGD was added to a constant volume of PacMaleimide LCL, Pac-LCL, and HEPES buffer $\mathrm{pH} 6.5,50 \mathrm{mM}$. The reaction was performed in triplicates at three different amounts of cRGD. After the incubation period of $12 \mathrm{~h}$. at $25^{\circ} \mathrm{C}$ for the conjugation reaction, the samples were digested with absolute ethanol, and the free cRGD remaining was quantified by RPHPLC method. The results indicate mean \pm standard deviation of three reactions (- Maleimide-Pac-LCL; $\triangle$ PacLCL; • HEPES buffer $\mathrm{pH} 6.5,50 \mathrm{mM}$ ). 
cRGD than required for maleimide-Pac-LCL. The Pac-LCL, and the reaction buffers were used as controls for non-specific binding, and total unbound respectively.

\subsubsection{Characteristics of Pac-RGD-GFTLCL}

The Pac-RGD-GFTLCL was prepared using Pac-RGD-TLCL similar to the procedure for the preparation of Pac-GFLCL. The amount of entrapped paclitaxel in both the formulations after reconstitution was similar, and was approximately $500 \mu \mathrm{g} / \mathrm{mL}$ with more than $90 \%$ of paclitaxel retained in the liposomes. The particle size of the Pac-RGDGFTLCL was also similar to Pac-GFLCL with an average diameter between 150-200 nm which is favorable for longer circulation time. These liposomes can escape RES, and also benefit from passive targeting due to EPR effect. The zeta potential of Pac-GFLCL and Pac-RGD-GFTLCL was $-30.80 \pm 6.95$ and $-35.07 \pm 1.55 \mathrm{mV}$ respectively. The zeta potential values of approximately $\sim-30 \mathrm{mV}$ suggest that these vesicles have adequate repulsive forces for better colloidal stability. The moisture content of the dried cake was less than $1 \% \mathrm{w} / \mathrm{v}$. The lyophilized cake exhibited acceptable physical properties with respect to appearance, cake integrity, cake height vs volume, and ease of reconstitution. The TEM images showed that both Pac-GFLCL and Pac-RGD-GFTLCL were spherical in structure (Figure 4-12).

\subsubsection{Visualization and Disintegration of Pac-RGD-GFTLCL with Ultrasound}

The ability to visualize and disintegrate the Pac-RGD-GFTLCL with ultrasound was evaluated using MTV-1 tail vein infusion catheter mounted in a rectangular plexiglass tank filled with water. The effect of high intensity ultrasound pulses on contrast mean power in the ROI is shown in Figure 4-13. The Pac-RGD-GFTLCL were visualized with ultrasound in the catheter which is about $0.2 \mathrm{~mm}$ internal diameter suggesting that these gas-filled liposomes can withstand shearing forces in the blood vessels. With a continuous flow of the liposomes through the catheter, the disintegration of the gas-filled liposomes was not perceivable due to rapid reperfusion in the ROI. So, the flow was stopped for the measurement sequence to determine the effect of high intensity ultrasound pulses on the Pac-RGD-GFTLCL. The contrast mean power decreased after application of ultrasound pulses indicating that these liposomes were disintegrated in the ROI. The contrast mean power increased to baseline within few seconds after application of high intensity ultrasound pulses due to reperfusion of fresh Pac-RGD-GFTLCL to the ROI. Even when the flow was stopped, reperfusion occurred which could be attributed to the Brownian motion of these particles of colloidal nature. We also made attempts to demonstrate visualization and disintegration of these gas-filled liposomes using subcutaneous breast cancer model in mice. We experienced difficulties in visualizing these liposomes in vivo. The loss of signal in the animal model could be the result of several factors. One reason is the presence of fewer barriers when these gas-filled liposomes were tested by an in vitro model in which the signals were received by the transducer with no or minimal attenuation. In contrast, the animal model presents several barriers that can affect signal to attenuation ratio. The second reason could be the ability 


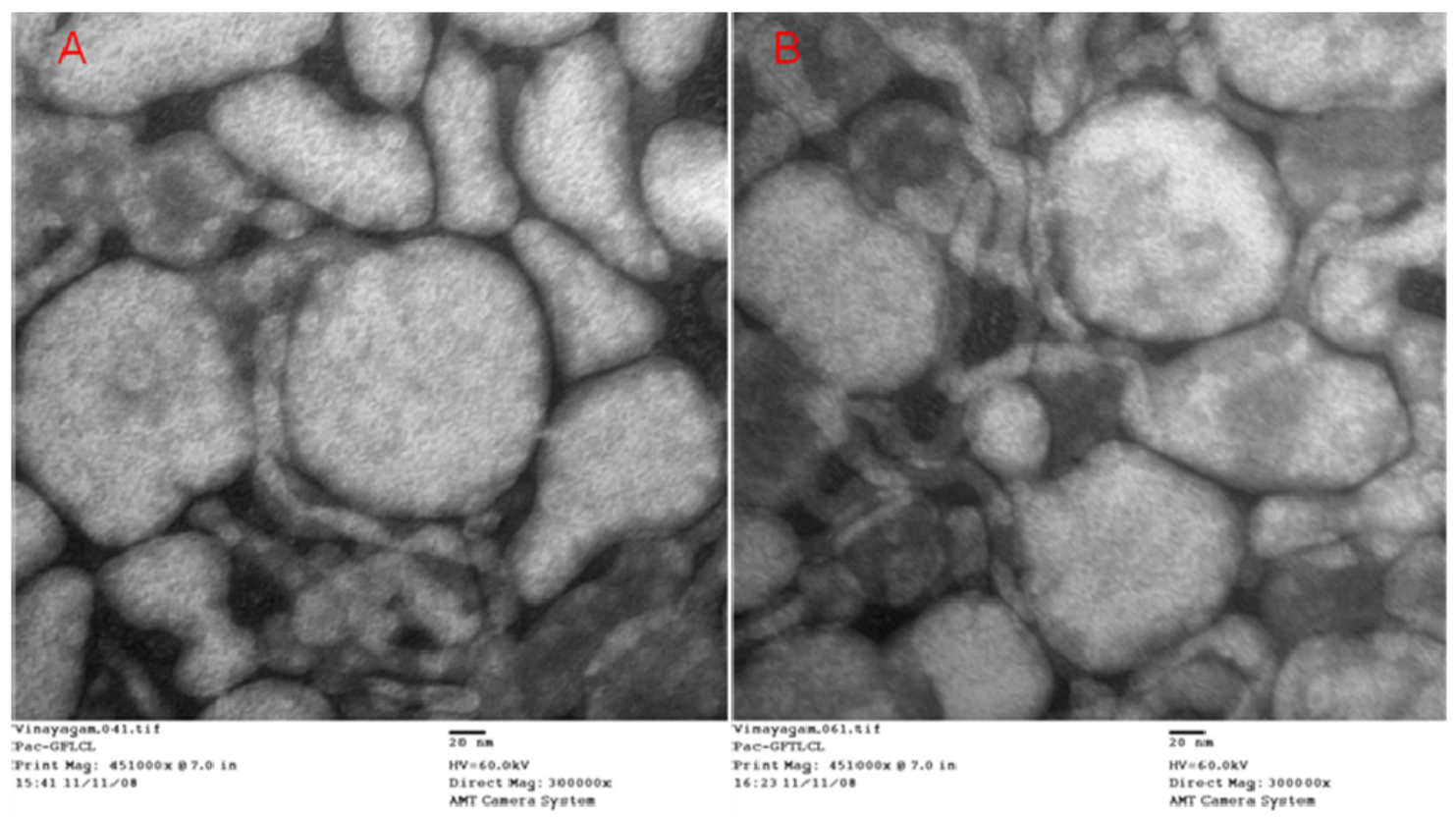

Figure 4-12. TEM images of Pac-GFLCL (A) and Pac-RGD-GFTLCL (B)

Notes. The samples were prepared by negative staining with uranyl acetate using sequential two drop technique on formvar coated copper grids. The results are shown at a magnification of $300,000 x$. 


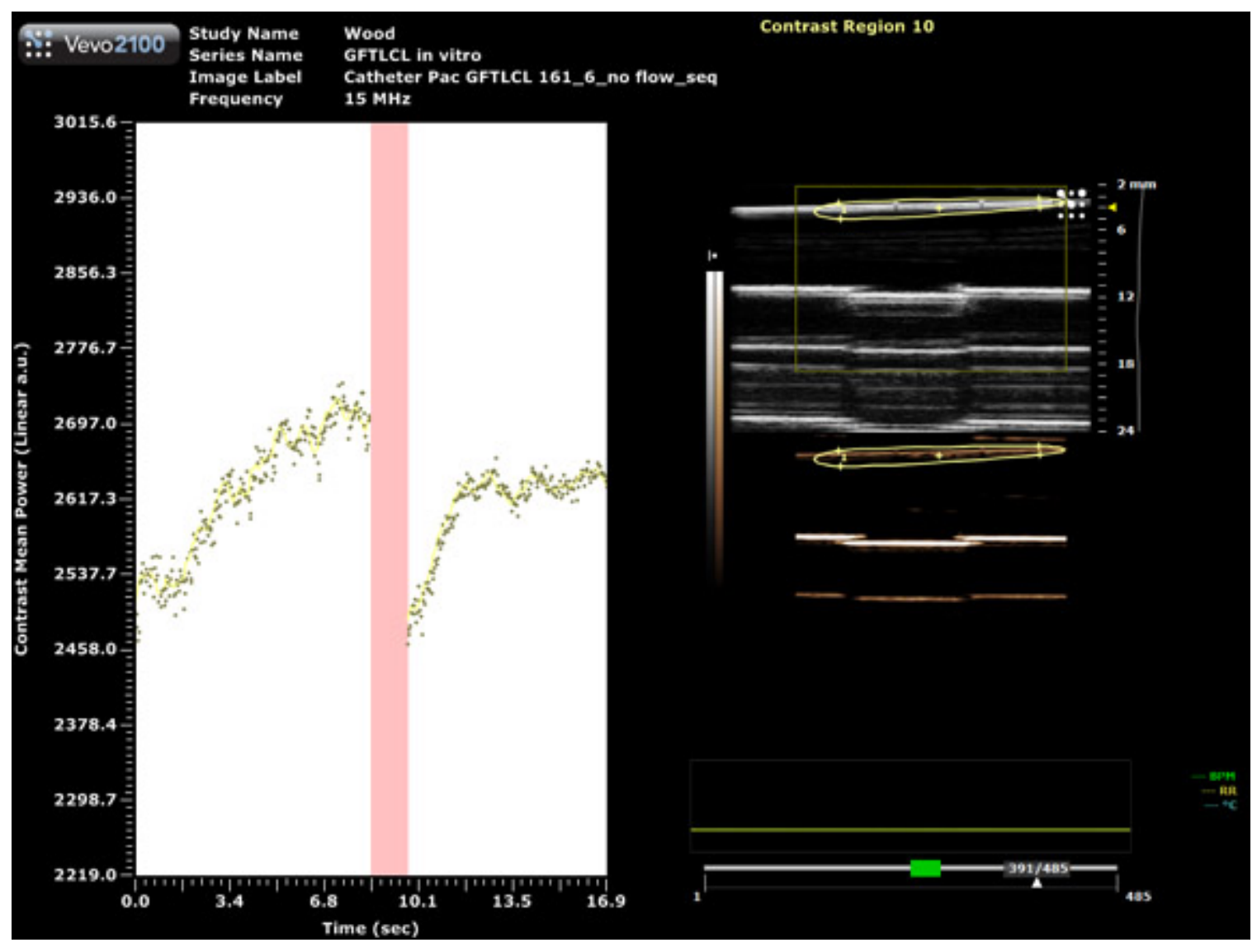

Figure 4-13. Disintegration of Pac-RGD-GFTLCL with ultrasound pulses

Notes. The Pac-RGD-GFTLCL was reconstituted with HPLC grade water and drawn into $1 \mathrm{cc}$ syringe. The syringe was connected to infusion catheter and the sample was slowly injected to fill the catheter. A cine loop sequence was started with high intensity pulse occurring at $50 \%$ of the loop for a duration of $1 \mathrm{sec}$. The cine loop was processed to calculated contrast mean power in the ROI. 
of the transducer to resolve these nano-sized liposomes. Interference from the tissue can make it even more difficult to resolve these liposomes. The third reason could be the dilution of these liposomes in the blood stream reducing the concentration available at the target site for visualization. The amount of gas present in each liposome, and the number of liposomes containing certain amount of gas in a given sample of gas-filled liposomes can also affect the average intensity received by the transducer. From the formulation development point of view, the liposomal bilayer should have adequate rigidity to retain gas in the bilayer in presence of shearing forces and blood pressure experienced in vivo.

The resonant frequency of nano-sized bubbles is in the range of 25-50 $\mathrm{MHz}$ [262]. But, the theoretical predictions of resonant frequency are based on assumption that the bubbles exhibit linear behavior. The predictions of harmonic generation from submicron ultrasound contrast agents by Zheng et al shows that the nanobubbles of size $\sim 200 \mathrm{~nm}$ exhibit non-linear behavior at frequencies relevant to clinical ultrasound instruments [263]. The same reason also explains the high backscatter obtained in the in vitro experiments at clinically relevant frequencies. The question of whether or not these gasfilled liposomes can be used to produce cavitation induced effect in vivo with clinical ultrasound instruments is still debatable. The use of correlation imaging can substantiate the data and help in demonstrating cavitation induced effects [264]. Nevertheless, these gas filled liposomes can be used for ultrasound mediated drug delivery, because the radiation forces can bring these liposomes closer to the cell surface and improve the chances of uptake by the cells $[265,266]$.

\subsubsection{Stability of Pac-RGD-GFTLCL}

The stability of the Pac-RGD-GFTLCL in $2 \mathrm{cc}$ amber vials was evaluated at $5 \pm 3^{\circ} \mathrm{C}$ for three months. The moisture content of the formulation was maintained below $1 \% \mathrm{w} / \mathrm{v}$ through the evaluation period. The cake retained acceptable physical properties of appearance, and ease of reconstitution. The entrapped paclitaxel content, percent paclitaxel retained, PSD, and zeta potential were evaluated in reconstituted samples. The stability data is presented in Figure 4-14. The Pac-RGD-GFTLCL maintained their size, charge, and integrity through the evaluation period. Paclitaxel content and percent paclitaxel retained in the liposomes was within $\pm 10 \%$ of the initial values suggesting that the formulation has adequate stability.

\subsection{SUMMARY AND CONCLUSIONS}

The formulation and process parameters in the development of paclitaxel-loaded gasfilled liposomes were identified and optimized. The particle size of the paclitaxel-loaded liposomes increased more than four-fold after lyophilization. The lipid-sugar ratio played a key role in controlling the particle size increase during lyophilization in the preparation of Pac-GFLCL, and the optimum lipid-sugar ratio was found to be 1:7. Inclusion of mannitol in the formulation caused an increase in PSD, and PDI of the liposomes after lyophilization. The mannitol containing Pac-GFLCL formulations showed higher 


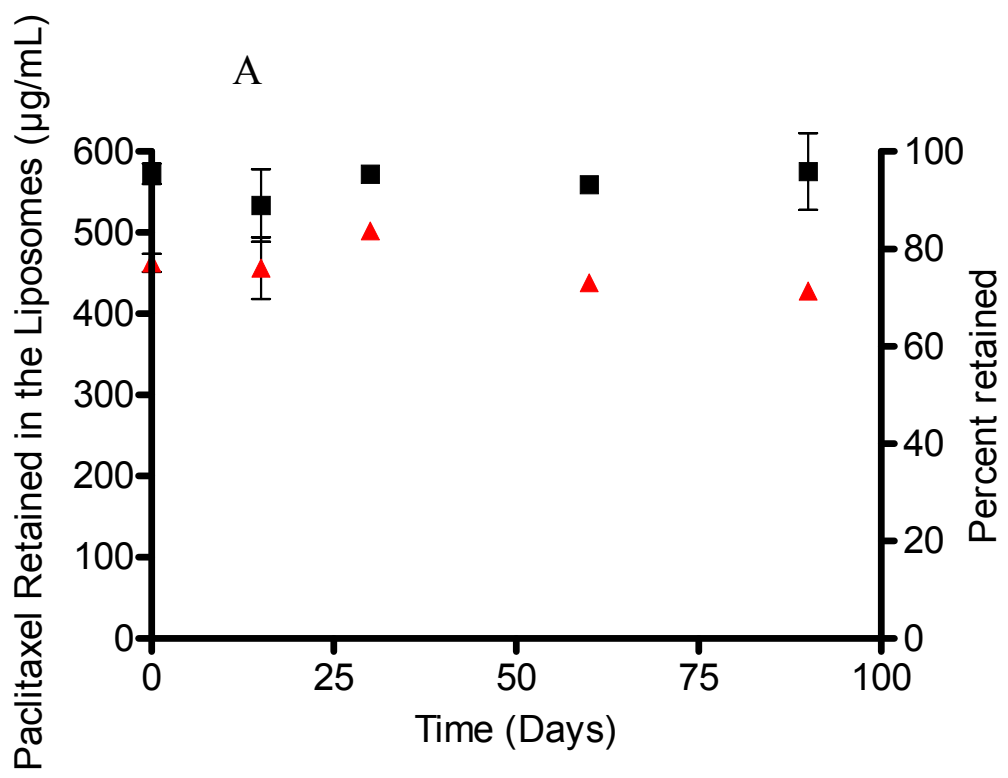

$\mathrm{B}$

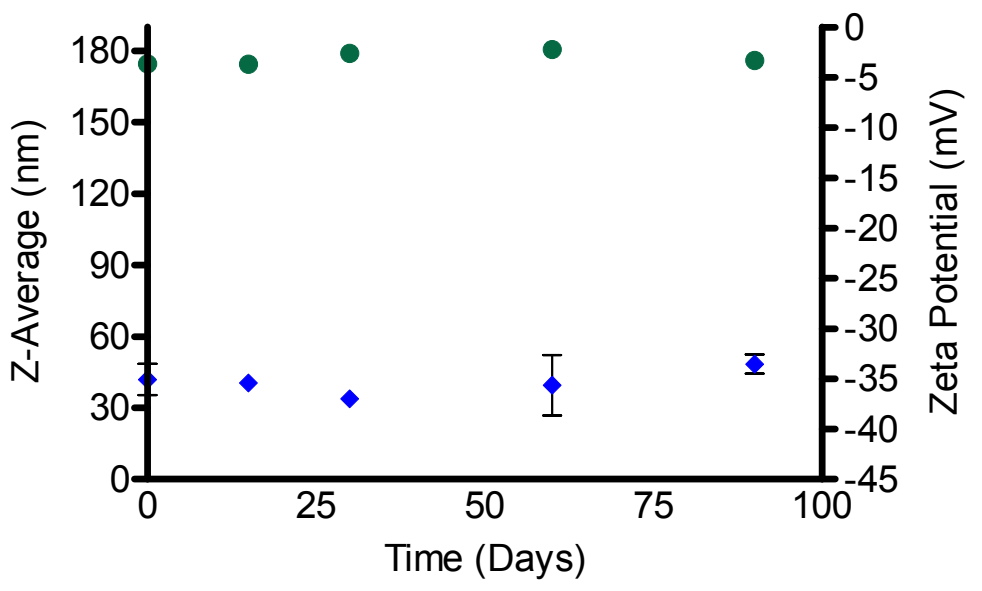

Figure 4-14. Stability of Pac-RGD-GFTLCL

Notes. The Pac-RGD-GFTLCL was stored at $5 \pm 3^{\circ} \mathrm{C}$ in $2 \mathrm{cc}$ amber vials. Samples were withdrawn at specific intervals, and reconstituted with HPLC water and analyzed. A. For the determination of paclitaxel retention, the samples were subjected to minicolumn centrifugation in Sephadex G-25 macro spin columns to separate free paclitaxel from total paclitaxel. The eluate containing entrapped paclitaxel was digested with ethanol, and quantified by RPHPLC method. The results indicate the mean \pm standard deviation for three gel columns ( $\Delta$ Paclitaxel concentration; a Percent paclitaxel retained in the liposomes). B. For PSD, and zeta potential, the results indicate mean \pm standard deviation of three independent measurements. The zeta potential results at 15 , and 30 days indicate a single measurement (• PSD; $\diamond$ Zeta potential). 
echogenicity than the formulation without mannitol. In spite of its positive effect on the echogenicity, mannitol was excluded from the formulation to have a better control of the PSD, and to avoid possible decrease in the in vivo circulation time due to slightly larger size. Nevertheless, mannitol containing formulations also have the potential for future development for their higher acoustic activity.

We have evaluated an indirect method for determining conjugation efficiency of cRGD to preformed liposomes containing reactive maleimide functional groups on the surface. The thioether bond is formed readily by covalent conjugation of sulfhydryl group and maleimide group. The conjugation can be verified by mass, and nuclear magnetic resonance spectroscopic methods in the pure form of the conjugate. But, it is difficult to quantify the conjugation efficiency by these direct methods when the peptide is conjugated to maleimide functional groups present on the surface of preformed liposomes. The RPHPCL method can quantify free cRGD, and so we quantified unreacted cRGD as a measure of conjugation efficiency. We have shown that there is non-specific binding of cRGD to the liposome surface and it can be saturated. The conjugation efficiency of cRGD to maleimide-Pac-LCL increased with an increase in the amount of cRGD added in the reaction. Thus, a slightly excess of cRGD than needed for the reaction is required to saturate non-specific binding, and to improve conjugation efficiency.

The Pac-RGD-GFTLCL was similar to Pac-GFLCL in terms of size, charge, echogenicity, and entrapped paclitaxel content. The Pac-RGD-GFTLCL was stable for three months at $5 \pm 3^{\circ} \mathrm{C}$ and maintained size, charge and integrity through the evaluation period. The in vitro experiments using mouse tail vein infusion catheter showed that PacRGD-GFTLCL can be visualized by ultrasound, and can be disintegrated with high intensity ultrasound pulses. The paclitaxel-loaded gas-filled targeted liposomes has the potential for improving the efficacy of the treatment of breast cancer by improving the uptake of the liposomes due to improved vascular permeability caused by disintegration using high intensity ultrasound pulses. While localized ultrasound pulses can be used for improving the therapy of tumors accessible by ultrasound using Pac-RGD-GFTLCL, the targeted nature of the liposomes can improve the therapy of tumors metastasized to other tissues and organs not accessible by ultrasound. The unique nature of this parenteral formulation has the potential to overcome the barriers in chemotherapy of drugs to solid tumors. 


\section{CHAPTER 5. IN VIVO EVALUATION OF BIODISTRIBUTION AND EFFICACY OF TUMOR VASCULATURE TARGETED LIPOSOMES AND COMPARISON OF EFFICACY WITH TUMOR CELL TARGETED LIPOSMES}

Cancer is the second leading cause of death in United States $[2,3]$ with about 1.5 million new cases and more than half-a-million deaths estimated for the year 2009 [4]. Solid tumors account for more than 85 percent of all the cases reported with cancer $[5,6]$. Breast cancer is the most commonly diagnosed form of solid tumors and is the second leading cause of death in western women. About $20-30 \%$ women with breast cancers develop metastatic breast cancers. Paclitaxel, one of the most effective drugs used in chemotherapy of breast cancer, is a natural product isolated from bark of the Pacific Yew tree, Taxus brevifolia $[8,9]$. It exhibits cytotoxic activity by promoting the assembly of microtubules from tubulin dimers and stabilizes microtubules by preventing depolymerization. Paclitaxel is marketed as Taxol ${ }^{\mathbb{R}}$ and Abraxane ${ }^{\mathrm{TM}}$ as an adjuvant in the treatment of node-positive breast cancer or second line treatment of breast cancer after failure of combination chemotherapy for metastatic disease or relapse within 6 months of adjuvant chemotherapy. Due to poor aqueous solubility of paclitaxel, Taxol ${ }^{\mathbb{B}}$ was formulated with ethanol and Cremophor ${ }^{\circledR} \mathrm{EL}$. Although the product is diluted before use, the concentration of Cremophor ${ }^{\mathbb{B}} \mathrm{EL}$ is high enough to cause serious hypersensitivity reactions on administration requiring pre-medication with corticosteroids and antihistamines [15]. Abraxane ${ }^{\mathrm{TM}}$, albumin-bound nanoparticle formulation of paclitaxel, addressed solvent related adverse effects of Taxol ${ }^{\circledR}$ and eliminated the need for premedication with corticosteroids. But, phase III clinical trials in metastatic breast cancer patients showed higher rates of sensory neuropathy compared to Taxol ${ }^{\mathbb{}}$ treatment [16]. Apart from the toxicity associated with the formulations, administration of free paclitaxel itself is associated with systemic toxicities such as myelosuppression, and peripheral neuropathy [17]. It is imperative to deliver paclitaxel locally to the tumor sites to minimize the exposure of normal tissues with free paclitaxel, and to reduce systemic toxicities. Hence, there is a need for an effective drug delivery system for chemotherapy with paclitaxel to produce cytotoxic concentrations of the drug at the tumor sites without affecting normal tissues.

Liposomes are biocompatible carriers with ability to incorporate both lipophilic and hydrophilic drugs [23]. Encapsulating drugs within vesicles has the potential advantage of higher drug carrying capacity compared to attaching them to single polymer chain [250]. Preparation of liposomes of paclitaxel can alter the pharmacokinetic and pharmacodynamic properties of the free drug and result in reduced toxicity and enhanced efficacy of the treatment [251]. The maximum tolerated dose of paclitaxel has been shown to increase with the liposomal formulation compared to the administration of Taxol $^{\circledR}$ [252]. The liposomal formulation of paclitaxel was also effective in minimizing the percent increase in the tumor size of paclitaxel resistant Colon 26 cancer in mice $[251,253]$, suggesting that the development of liposomes can be used a strategy to overcome drug resistance in cancer. Conventional liposomes are cleared rapidly by the reticuloendothelial system (RES) comprised of Kupffer cells of the liver, and fixed macrophages of the spleen [80]. Clearance of liposomes by the RES is directly 
proportional to the vesicle size [79]. Liposomes of size $\sim 100 \mathrm{~nm}$ can escape clearance by the RES and remain in circulation for a prolonged period of time. In addition, coating the surface of the liposomes with hydrophilic polymers such as PEG can provide a shielding effect to the liposomes and prevent recognition and binding of opsonins [88]. Therefore, these long-circulating liposomes can escape clearance by the RES and remain in circulation for a longer period of time. If the liposomes remain in circulation for a longer time, the possibility of their passive accumulation in the tumors by enhanced permeability and retention (EPR) effect will be improved. Such passive targeting of liposomes at the tumors does not necessarily elicit an improved therapeutic response which is dependent on the cellular uptake of the liposomes. Cellular uptake can be improved by an active targeting approach. Active targeting refers to the attachment of specific targeting ligands to the therapeutic agent or carrier system to recognize and interact with the target receptors or proteins at the cellular or tissue components [20, 107, 116]. Although similar biodistribution and tumor accumulation is observed with liposomes by passive targeting as well as active targeting, an enhanced therapeutic response is accomplished with active targeting of liposomes directed against specific receptors due to improved cellular uptake $[117,118]$.

Endothelial cells within angiogenic blood vessels of solid tumors express several proteins which are either absent or expressed at very low levels in well developed normal blood vessels [161-163]. The $\alpha_{v} \beta_{3}$ integrin receptors are highly expressed in actively proliferating endothelial cells of tumor vasculature and tumor cells [163, 235]. Peptides with Arg-Gly-Asp (RGD) motif in a cyclic framework have been shown to bind to these receptors and are effective in targeting solid tumors[172]. By attaching RGD peptide to the surface of the paclitaxel-loaded liposomes through PEG spacers, paclitaxel can be delivered specifically at the tumor site minimizing its exposure to normal tissues. An attractive advantage of targeting tumor vasculature is that the tumor blood vessels are readily available for targeting than the tumor cells and thus specific targeting can be achieved.

Iron $(\mathrm{Fe})$ is required in the active sites of the enzyme ribonucleotide reductase during DNA synthesis [138]. Due to redox activity and its potential to generate reactive oxygen species (ROS), the amount of free Fe is kept at minimum levels [139]. To control the amount of unbound $\mathrm{Fe}$, the metal is transported bound specifically to the two high affinity binding sites of serum glycoprotein, transferrin (Tf). The uptake of Fe by the cells involves binding of the Fe-loaded Tf to transferrin receptor1 (TfR1) present on the cell surface. Binding induces receptor mediated endocytosis of Fe-Tf [140]. TfR1 is a glycoprotein expressed in all nucleated cells at low levels [141]. The density of TfR1 on the surface of cells is correlated with the extent of cell growth and division, and consequently, the tumor cells express more TfR1 than normal cells. Thus, the expression of TfR 1 is elevated in proliferating cells which makes these receptors excellent choice for targeted delivery to cancer [142]. By attaching Tf to the surface of the paclitaxel-loaded liposomes through PEG spacers and linkers, paclitaxel can be delivered specifically to the tumor cells over expressing TfR1. 
The objective of this work is to develop paclitaxel-loaded RGD conjugated targeted long-circulating liposomes (Pac-RGD-TLCL). The biodistribution of these tumor vasculature targeted liposomes was evaluated by non-invasive near infrared fluorescence (NIRF) imaging using DiR-loaded RGD conjugated targeted long-circulating liposomes (DiR-RGD-TLCL) in mouse xenografts bearing MDA-MB-231 breast cancer. The biodistribution was also verified by quantifying paclitaxel in plasma and tumor tissues 24 hours after the administration of paclitaxel-loaded liposomes. The antitumor efficacy of these tumor vasculature targeted liposomes were evaluated in comparison to tumor cell targeted paclitaxel-loaded transferrin conjugated targeted long-circulating liposomes (Pac-Tf-TLCL).

\subsection{MATERIALS AND METHODS}

\subsubsection{Materials}

Paclitaxel was procured from 21CEC Pharmaceuticals (Bradenton, FL). 1,1'dioctadecyl-3,3,3',3'-tetramethylindotricarbocyanine iodide (DiR) was obtained from Molecular Probes (Eugene, OR). The phospholipids used in this work were all of research grade. Egg phosphatidylcholine (EPC), hydrogenated soy phosphatidylcholine (HSPC) and 1,2-distearoyl-sn-glycero-3-phoshoethanolamine-N-(poly(ethylene glycol)2000) (DSPE-PEG ${ }_{2000}$ ) were obtained from Northern Lipids (Burnbay, BC, Canada). 1,2-distearoyl-sn-glycero-3-phoshoethanolamine-N-(maleimide poly(ethylene glycol)2000) (maleimide DSPE-PEG 2000 ) and 1,2-distearoyl-sn-glycero-3phoshoethanolamine-N-(biotinyl poly(ethylene glycol)2000) (biotinyl DSPE-PEG 2000 ) were obtained from Avanti Polar Lipids (Alabaster, AL). Cyclo (Arg-Gly-Asp-D-PheCys) peptide (cRGD) was obtained from Peptides International (Louisville, KY). Biotin labeled human transferrin (Tf), cholesterol and $\alpha$-tocopherol were purchased from Sigma (St. Louis, MO). Streptavidin was obtained from PROzyme (Hayward, CA). Dulbecco's Modified Eagle Medium (D-MEM) with high glucose, heat inactivated fetal bovine serum (FBS), penicillin-streptomycin (10,000 U/mL Penicillin G, 10,000 $\mu \mathrm{g} / \mathrm{mL}$ streptomycin), and $0.25 \%$ Trypsin EDTA were obtained from Invitrogen (Carlsbad, CA). BD Matrigel ${ }^{\mathrm{TM}}$ basement membrane matrix was obtained from BD Biosciences (Bedford, MA). HPLC grade water, acetonitrile, and methanol were obtained from Fisher Scientific (Fair Lawn, NJ). HPLC grade chloroform was purchased from Acros Organics (Morris Plains, NJ). All other chemicals and reagents were of analytical grade and used without further purification or characterization.

\subsubsection{Cell Culture}

MDA-MB-231 human breast cancer cell lines were obtained from American Type Culture Collection (ATCC, Manassas, VA). The cells were cultured in D-MEM supplemented with $10 \% \mathrm{v} / \mathrm{v}$ FBS, and $1 \% \mathrm{v} / \mathrm{v}$ antibiotics $(100 \mathrm{U} / \mathrm{mL}$ of penicillin $\mathrm{G}$ and 
$100 \mu \mathrm{g} / \mathrm{mL}$ of streptomycin). The cells were maintained in an incubator at $37^{\circ} \mathrm{C}$ in a humidified atmosphere, and 5\% carbon dioxide.

\subsubsection{Preparation of Long-Circulating Liposomes Loaded with DiR (DiR LCL) or Paclitaxel (Pac-LCL)}

The DiR LCL or Pac-LCL was be prepared by the lipid film hydration-extrusion method using EPC, HSPC, DSPE-PEG 2000 , and cholesterol. The lipid film was prepared by dissolving DiR or paclitaxel, phospholipids, cholesterol, and $\alpha$-tocopherol in 9:1 chloroform-methanol. The solution obtained was evaporated in a round bottom flask using rotary evaporator under reduced pressure at $45^{\circ} \mathrm{C}$ for $4-6 \mathrm{~h}$. The dry film was hydrated with $50 \mathrm{mM}$ HEPES buffer $\mathrm{pH} 6.5$ at $65^{\circ} \mathrm{C}$ for $45-60$ minutes. The multilamellar vesicles (MLVs) thus obtained were sized by extrusion through stacked polycarbonate membrane filters of varying pore diameters under high pressure to obtain small unilamellar vesicles (SUVs). The SUVs were characterized for particle size distribution, zeta potential, and total and entrapped DiR or paclitaxel content. DiR was quantified by spectrofluorimetry, and paclitaxel was quantified by reversed phase high performance chromatography (RPHPLC). The liposomes were sterilized by filtration through $0.22 \mu \mathrm{m}$ membrane filter in an aseptic environment and stored in light protected vials at $5 \pm 3^{\circ} \mathrm{C}$ until further use.

\subsubsection{Preparation of cRGD Conjugated Targeted Long-Circulating Liposomes Loaded with DiR (DiR-RGD-TLCL) or Paclitaxel (Pac-RGD-TLCL)}

For the preparation of DiR-RGD-TLCL or Pac-RGD-TLCL, a part (0.5 moles \%) of DSPE-PEG $_{2000}$ was replaced with maleimide DSPE-PEG 2000 and the liposomes were prepared as described in section 5.1.3 to obtain SUVs with reactive maleimide functional groups. The maleimide-DiR-LCL or maleimide-Pac-LCL thus obtained was conjugated with cRGD peptide by incubation at $25^{\circ} \mathrm{C}$ for $12 \mathrm{~h}$. The reaction was quenched by the addition of 2-mercaptoethanol to block free maleimide. The unconjugated peptide and 2mercaptoethanol remaining in reaction mixture were removed from the liposomes by equilibrium dialysis through 10,000 MWCO Slide-A-Lyzer dialysis cassettes (Thermo Fisher Scientific, Rockford, IL) against $50 \mathrm{mM}$ HEPES buffer $\mathrm{pH} 6.5$ at $5 \pm 3{ }^{\circ} \mathrm{C}$ overnight. The liposomes were sterilized by filtration through $0.22 \mu \mathrm{m}$ membrane filter in an aseptic environment and stored in light protected vials at $5 \pm 3{ }^{\circ} \mathrm{C}$ until further use.

\subsubsection{Preparation of Paclitaxel-Loaded Transferrin Conjugated Targeted Long- Circulating Liposomes (Pac-Tf-TLCL)}

For the preparation of Pac-Tf-TLCL, a part (0.01 moles \%) of DSPE-PEG 2000 was replaced with biotinylated DSPE-PEG 2000 and the liposomes were prepared similar to 5.1.3 using phosphate buffered saline $\mathrm{pH} 7.2$ (PBS) instead of HEPES buffer. Transferrin was non-covalently conjugated to biotin on the distal end of the PEG spacers of the 
biotinylated SUVs using the procedure established in our laboratory [159]. Briefly, streptavidin was dissolved in PBS to prepare a $2 \mathrm{nM}$ solution. To the streptavidin solution in a scintillation vial, biotinylated liposomes was added, and mixed. The reaction mixture was incubated at $25^{\circ} \mathrm{C}$ for $30 \mathrm{~min}$, and the free streptavidin was removed by ultrafiltration using Centricon 100 centrifugal filter device (100 KD MWCO, Millipore, Bedford, MA) at $5000 \mathrm{~g}$ for $30 \mathrm{~min}$ to obtain purified streptavidin conjugated long-circulating liposomes (SALCL). Biotin labeled human transferrin was dissolved in PBS (2 nM) and added to the SALCL, incubated at $25^{\circ} \mathrm{C}$ for $30 \mathrm{~min}$, and the free transferrin was removed by ultrafiltration. The liposomes were sterilized by filtration through $0.22 \mu \mathrm{m}$ membrane filter in an aseptic environment and stored in light protected vials at $5 \pm 3^{\circ} \mathrm{C}$ until further use.

\subsubsection{Characterization of Liposomes}

The average diameter (Z-avg) of the liposomes was determined using dynamic light scattering using Zetasizer Nano ZS (Malvern Instruments, Westborough, MA) equipped with $4.5 \mathrm{~mW}$ diode laser as a source of light operating at $670 \mathrm{~nm}$. Particle scattered photons were detected at an angle of $173^{\circ}$. The samples were suitably diluted with HPLC grade water for the determination of Z-avg. Three independent measurements were performed for each sample. Zeta potential was determined from electrophoretic mobility using samples suitably diluted with HPLC grade water.

The loading efficiency of DiR or paclitaxel in the liposomes was determined by minicolumn centrifugation method using Sephadex G-25 macro spin columns (Harvard apparatus, Holliston, MA) similar to the method described by Zhang et al [249] with modifications. Briefly, the Sephadex G-25 particles in the column were suspended in $0.1 \% \mathrm{w} / \mathrm{v}$ TFA and were allowed to swell for 15 minutes. The columns were centrifuged at $1500 \mathrm{~g}$ at $25^{\circ} \mathrm{C}$ for 4 min using IEC CL31R Multispeed Centrifuge (Thermo Scientific, Asheville, NC) to remove TFA. About $100 \mu \mathrm{L}$ of placebo LCL was added and centrifuged to saturate the column. The column saturation with placebo liposomes was repeated for a total of three times. The gel columns were placed in fresh collection tubes for use with drug-loaded liposomes. The liposome samples for analysis were diluted with the dispersion medium of the liposomes. About $100 \mu \mathrm{L}$ of the diluted sample was added to the gel column, and centrifuged for 4 minutes. The eluate in the collection tube, containing only entrapped DiR or paclitaxel was analyzed by spectrofluorimetry or RPHPLC respectively. The total DiR or paclitaxel in the liposomes was determined form samples before passing through Sephadex G-25 columns. For quantification of DiR, the liposomes were digested with $70 \%$ isopropanol and the concentration of DiR was determined in 96 well black non transparent microplates by spectrofluorimetry using SpectraMax M2e Microplate/Cuvette Reader (Molecular Devices, Sunnyvale, CA) at the excitation wavelength of $750 \mathrm{~nm}$ and the emission wavelength of $780 \mathrm{~nm}$. For quantification of paclitaxel, the liposomes were digested with absolute ethanol, and suitably diluted before injection to the HPLC system. The HPLC system comprised of Waters 600 Controller, Waters 717 Plus Autosampler, and Waters 2996 Photodiode Array Detector (Waters Corporation, Milford, MA) was used. The RPHPLC analysis was 
performed using a Nova-Pak C18 column $(3.9 \times 150 \mathrm{~mm}, 4 \mu \mathrm{m})$ (Waters Corporation, Milford, MA). An isocratic elution was performed using acetonitrile-water in a ratio 55: $45 \mathrm{v} / \mathrm{v}$ at a flow rate of $1 \mathrm{~mL} / \mathrm{min}$, and detection wavelength of $230 \mathrm{~nm}$. The encapsulation efficiency for DiR or paclitaxel was calculated using the formula, Entrapment efficiency $=($ Entrapped DiR or paclitaxel x 100)/Total DiR or paclitaxel $)$.

\subsubsection{Stability Evaluation of Pac-RGD-TLCL and Pac-Tf-TLCL}

Stability of Pac-RGD-LCL and Pac-Tf-TLCL was evaluated in $2 \mathrm{cc}$ amber glass vials at $5 \pm 3^{\circ} \mathrm{C}$ for three months. Samples were withdrawn at specific intervals and analyzed for total and entrapped paclitaxel, entrapment efficiency, PSD, and zeta potential.

\subsubsection{Breast Cancer Mouse Xenograft Model}

The animal studies were conducted in accordance with the protocols approved by the Institutional Animal Care and Use Committee (IACUC) of The University of Tennessee Health Science Center, Memphis, TN. Female athymic nude mice (Harlan Laboratories, Indianapolis, IN) of 5-6 weeks age were used for the development of human breast cancer xenograft model. The MDA-MB-231 cells were detached from approximately $90 \%$ confluent cultures by brief exposure to $0.25 \%$ trypsin EDTA. The harvested cells were washed, and suspended in serum free D-MEM. The cell viability was verified by trypan blue dye exclusion method, and the viable cell count was recorded. The cell suspension was centrifuged and the cell pellet was resuspended in serum free D-MEM to get $\sim 5 \times 10^{6}$ cells in $0.1 \mathrm{~mL}$. The resulting cell suspension was mixed with equal volume of cold Matrigel ${ }^{\mathrm{TM}}$ and mixed well. The mice were anesthetized with isoflurane prior to tumor inoculation, and $0.2 \mathrm{~mL}$ of the cell suspension-Matrigel ${ }^{\mathrm{TM}}$ mixture was injected subcutaneously into the breast tissue of each mouse in the right axillary region. The tumor size was measured with calipers twice a week, and the tumor volume was calculated using the formula $\mathrm{V}=0.5 \mathrm{ab}^{2}(\mathrm{~V}$ - tumor volume; $\mathrm{a}$ - largest diameter; $\mathrm{b}$ smallest diameter).

\subsubsection{Evaluation of Biodistribution of Tumor Vasculature Targeted Liposomes by Near Infrared Fluorescence (NIRF) Imaging}

To evaluate the localization of the tumor vasculature targeted cRGD conjugated liposomes, athymic nude mice bearing MDA-MB-231 breast cancer in the axillary region were used. The tumors were allowed to grow up to $200 \mathrm{~mm}^{3}$ to initiate the study. The animals were randomly divided into four groups of five mice each. The animals were anesthetized with isoflurane, and DiR Solution formulation (Prepared by dissolving DiR in 50: 50 Cremophor ${ }^{\circledR}$ ELP and Ethanol, and the resulting formulation was diluted to four times with normal saline prior to administration), DiR LCL, and DiR-RGD-TLCL containing $5 \mu \mathrm{g}$ of $\mathrm{DiR}$ in $\sim 50 \mu \mathrm{L}$ of the formulation, and normal saline as control was injected by retro-orbital injection. The animals were anesthetized with isoflurane and the 
whole body optical imaging was performed at 1, 4, 8, 24, 48, and $72 \mathrm{~h}$ using Xenogen IVIS $^{\circledR}$ Lumina imaging system (Caliper LifeSciences, Hopkinton, MA). The excitation wavelength was $745 \mathrm{~nm}$ and the emission wavelength was $780 \mathrm{~nm}$. The animals were maintained under isoflurane anesthesia by Xenogen X91-8 Gas Anesthesia System (Caliper LifeSciences, Hopkinton, MA) during the measurement. The fluorescence intensity in the region of interest (ROI) was calculated in tumor, peritoneal cavity, and flank tissue using Living Image ${ }^{\circledR}$ 3.2 Software (Caliper LifeSciences, Hopkinton, MA). The pixel intensity of the dye in tumor was compared to the intensity in the surrounding normal tissues as a measure of the concentration in the circulation. Tumor to normal tissue $(\mathrm{T} / \mathrm{N})$ accumulation ratio was calculated to estimate the tumor targeting index as a measure of localization of liposomes in the tumors. The mice were euthanized after 72 hours. The tumor, liver, and spleen were isolated and the images of the isolated organs were acquired.

\subsubsection{Evaluation of Biodistribution of Paclitaxel from Tumor Vasculature Targeted Liposomes}

To evaluate the effect of size, and tumor vasculature targeting on biodistribution of paclitaxel from liposomes, athymic nude mice bearing MDA-MB-231 breast cancer in the axillary region were used. The tumors were allowed to grow up to $200 \mathrm{~mm}^{3}$ to initiate the study. The animals were randomly divided into four groups of three mice each. The animals were anesthetized with isoflurane, and paclitaxel solution formulation (Prepared by dissolving paclitaxel in 50: 50 Cremophor ${ }^{\circledR}$ ELP and Ethanol, and the resulting formulation was diluted to four times with normal saline prior to administration), PacLCL (160 nm), and Pac-RGD-TLCL (160 nm), and Pac-RGD-TLCL (80 nm) containing at a total paclitaxel dose of $7.5 \mathrm{mg} / \mathrm{kg}$ was injected by retro-orbital injection. After $24 \mathrm{~h}$, the blood samples were collected by retro-orbital bleeding under anesthesia. The animals were euthanized and tumor tissues were collected, washed with normal saline and stored at $-20^{\circ} \mathrm{C}$ until analyzed. Plasma was separated from blood by centrifugation and stored at $-20^{\circ} \mathrm{C}$ until analyzed. The paclitaxel content from the plasma and tumor tissues were quantified by the RPHPLC method similar to the method described by Yang et al [260] with slight modifications. The paclitaxel from plasma was extracted by vortexing $50 \mu \mathrm{L}$ of plasma with $2 \mathrm{~mL}$ of ethyl acetate. The mixture was centrifuged for 5 minutes at 3000 $\mathrm{rpm}$. The supernatant was collected, and the extraction was repeated with another $2 \mathrm{~mL}$ of ethyl acetate. The supernatants containing paclitaxel were pooled and evaporated in a fume hood to dryness. The residue was dissolved in absolute ethanol, centrifuged for 5 minutes at $3000 \mathrm{rpm}$ and the supernatant containing paclitaxel was analyzed by the RPHPLC method described in 5.1.6. For quantification of tissue paclitaxel, the tumor tissues were homogenized in $2 \mathrm{~mL}$ of Dulbecco's phosphate buffered saline (DPBS) using Omni International 2000 tissue homogenizer (Omni International, Kennesaw, GA). The homogenate was extracted twice with 2-3 mL of ethyl acetate as described for plasma samples. The ethyl acetate fractions were combined and evaporated to dryness. The residue was dissolved in absolute ethanol, centrifuged for 5 minutes at $3000 \mathrm{rpm}$ and the supernatant containing paclitaxel was analyzed by the RPHPLC method described in 5.1.6. 


\subsubsection{Evaluation of Antitumor Efficacy of Paclitaxel-Loaded Tumor Vasculature Targeted Liposomes and Tumor Cell Targeted Liposomes}

The antitumor efficacy of tumor vasculature targeted Pac-RGD-TLCL and tumor cell targeted Pac-Tf-TLCL was evaluated in athymic nude mice bearing MDA-MB-231 breast cancer in the axillary region. The tumors were allowed to grow up to $100-200 \mathrm{~mm}^{3}$ to initiate the study. The tumor bearing animals were randomly divided into five groups of five mice each. The treatment groups were normal saline $(100 \mu \mathrm{L})$ as a control to evaluate the effect of no treatment, paclitaxel solution formulation (Prepared by dissolving paclitaxel in 50: 50 Cremophor ${ }^{\circledR}$ ELP and Ethanol, and the resulting formulation was diluted to four times with normal saline prior to administration) to evaluate the effect of Taxol ${ }^{\circledR}$ formulation as treatment, Pac-LCL formulation to evaluate the effect of passive targeting, Pac-RGD-TLCL to evaluate the effect of tumor vasculature targeting, and Pac-Tf-TLCL to evaluate the effect of tumor cell targeting. The animals were anesthetized with isoflurane and the formulations were injected by retroorbital injection at a paclitaxel dose of $7.5 \mathrm{mg} / \mathrm{kg}$ on 8.11 , and 14 days post tumor inoculation. The effect on tumor size was monitored for six weeks. The animals were euthanized when tumors reached $20 \mathrm{~mm}$ in diameter.

\subsubsection{Statistical Analysis}

The statistical analysis was performed by one-way analysis of variance (ANOVA) for paclitaxel tissue distribution study, and two-way ANOVA for NIRF imaging study and antitumor efficacy study using GraphPad Prism 3.0 Software (GraphPad Software, La Jolla, CA). Bonferroni post test analysis was performed to compare between the treatment groups. The results with $p<0.05$ were accepted as statistically significant.

\subsection{RESULTS AND DISCUSSION}

\subsubsection{Characterization of the Liposomal Formulations}

The characteristics of paclitaxel-loaded liposomal formulations are presented in Table 5-1. The paclitaxel loading in these formulations were between 1.0 to $1.2 \mathrm{mg} / \mathrm{mL}$ with entrapment efficiency greater than $90 \%$. The liposomal paclitaxel concentration thus achieved allowed dosing volumes similar to paclitaxel solution formulation $\left(\mathrm{Taxol}^{\mathbb{}}{ }^{\mathbb{}}\right)$ which is equivalent to $1.5 \mathrm{mg} / \mathrm{mL}$ when diluted with normal saline. The higher entrapment efficiency minimizes the amount of free drug in the formulation which will help minimize the systemic toxicities associated with the administration of free drug and its exposure to normal tissues. The vesicle size of these liposomes was within $100 \pm 20$ $\mathrm{nm}$ indicating that they can escape clearance by the RES. The PEG coating on the surface of these liposomes forms a hydrophilic coating on the surface which can prevent the binding of opsonins to the surface and thereby prevent recognition by the RES. Due to combined effect of size and PEG coating, these liposomes can remain in circulation 
Table 5-1. Characteristics of paclitaxel-loaded liposomes

\begin{tabular}{cccc}
\hline & \multicolumn{3}{c}{ Formulation } \\
\cline { 2 - 4 } Property of liposomes $^{\mathrm{a}}(\mathrm{Pac}-\mathrm{LCL}$ & Pac-RGD-TLCL & Pac-Tf-TLCL \\
\hline Total paclitaxel $^{\mathrm{a}}(\mu \mathrm{g} / \mathrm{mL})$ & $1249.12 \pm 1.16$ & $1165.75 \pm 1.89$ & $1153.32 \pm 1.91$ \\
Entrapped paclitaxel $^{\mathrm{b}}(\mu \mathrm{g} / \mathrm{mL})$ & $1153.21 \pm 2.41$ & $1073.36 \pm 0.65$ & $1085.29 \pm 4.42$ \\
Entrapment efficiency $^{\mathrm{c}}$ & 92.32 & 92.07 & 94.10 \\
Z-Average $^{\mathrm{d}}(\mathrm{nm})$ & $86.40 \pm 0.77$ & $91.29 \pm 0.19$ & $108.27 \pm 0.28$ \\
Zeta Potential $^{\mathrm{e}}(\mathrm{mV})$ & $-24.17 \pm 1.00$ & $-27.87 \pm 2.52$ & $-11.53 \pm 0.51$ \\
\hline
\end{tabular}

${ }^{a}$ The liposomes were digested with absolute ethanol and the concentration was determined by RPHPLC method. The results indicate mean \pm standard deviation of three injections.

${ }^{\mathrm{b}}$ The liposomes were subjected to minicolumn centrifugation in Sephadex G-25 macro spin columns to separate free paclitaxel from total paclitaxel. The eluate containing entrapped paclitaxel was digested with ethanol, and quantified by RPHPLC method. The results indicate mean \pm standard deviation of three injections.

${ }^{c}$ The entrapment efficiency was calculated from the formula, Entrapment efficiency $=$ (Entrapped paclitaxel x 100)/Total paclitaxel.

${ }^{\mathrm{d}}$ The samples were suitably diluted with HPLC grade water and measurements were made using dynamic light scattering method. The results indicate mean \pm standard deviation of three measurements.

${ }^{\mathrm{e}}$ The samples were suitably diluted with HPLC grade water and measurements were made from electrophoretic mobility. The results indicate mean \pm standard deviation of three measurements. 
for a longer time. The leaky nature of the tumor vessels and compromised lymphatic drainage together with long circulation property of these liposomes can improve the passive uptake of these liposomes in the tumor by the EPR effect. The liposomes have a slight negative charge which can improve the colloidal stability.

\subsubsection{Stability of Paclitaxel-Loaded Targeted Liposomes}

The stability of the Pac-RGD-TLCL and Pac-Tf-TLCL dispersions was evaluated at 5 $\pm 3^{\circ} \mathrm{C}$ in light protected container for three months. The stability data presented in Figures 5-1 and 5-2 shows that the liposomes maintained their size, charge, and integrity through the evaluation period. A similar trend of slight decrease in entrapped paclitaxel was observed with both targeted liposomes the first few days. This could be attributed to partitioning of the drug form the bilayer of liposomes to the dispersion medium to maintain equilibrium. Once the equilibrium is reached, the paclitaxel retention remained constant. The conjugation with either cRGD or transferrin did not affect the stability of the liposomes. The stability data is consistent with non targeted Pac-LCL formulation presented in Chapter 3.

\subsubsection{Near Infrared Fluorescence Imaging}

Near infrared fluorescence (NIRF) imaging is a non-invasive whole body imaging technique that can offer valuable information on biodistribution and tumor targeting ability [267, 268]. NIRF imaging techniques are cost effective, have high detection sensitivity, and use non-ionizing radiation $[269,270]$. Imaging performed at the NIR region can also minimize auto fluorescent signal of tissue which increases at wavelengths less than $600 \mathrm{~nm}$. Inclusion of a fluorescent dye that can be imaged by NIRF imaging in the bilayer of liposomes will allow direct visualization of kinetics and localization of the liposomes in a non-invasive manner. So, DiR, a lipophilic carbocyanine dye was loaded in the bilayer of LCL and RGD-TLCL to evaluate the biodistribution of these liposomes in mouse xenografts bearing breast cancer. The DiR-LCL, and DiR-RGD-TLCL were of the size $88.30 \pm 1.21 \mathrm{~nm}$, and $86.45 \pm 0.33 \mathrm{~nm}$ respectively. The zeta potential of DiRLCL, and DiR-RGD-TLCL were $-25.63 \pm 0.57 \mathrm{mV}$, and $-26.73 \pm 0.76 \mathrm{mV}$ respectively. These liposomes were similar to paclitaxel-loaded liposomes in size, charge, and composition. The in vivo circulation of these DiR-loaded liposomes are expected to represent the fate of paclitaxel-loaded liposomes. Since NIRF imaging is non-invasive in nature, localization of the liposomes in the tumor tissues can be determined at several time points in the same animal without the need sacrifice the animal. The kinetic data thus obtained can be extrapolated to determine the fate of paclitaxel-loaded liposomes with the assumption that DiR and paclitaxel-loaded liposomes behave in a similar manner. In this study, DiR-loaded liposomal formulations were compared with DiR micellar solution formulation as a positive control, and normal saline as a negative control. Representative images from each group at different time points are presented in Figure 5-3. The DiR solution formulation showed maximum accumulation in the tumor within $8 \mathrm{~h}$ of administration and the intensity decreased with time till $72 \mathrm{~h}$ time point. 


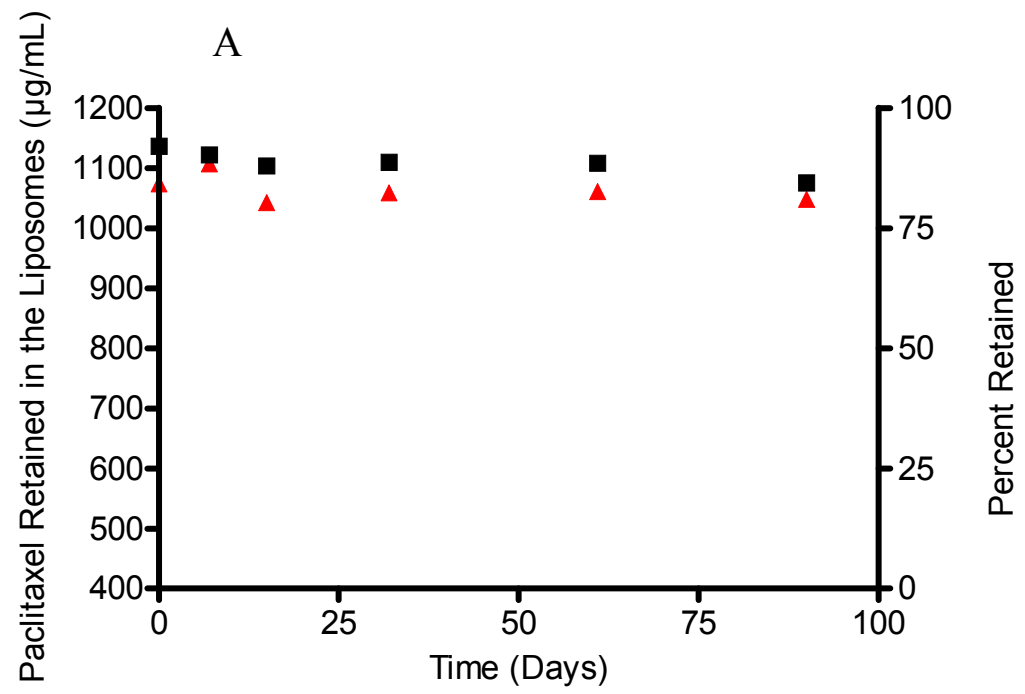

$\mathrm{B}$

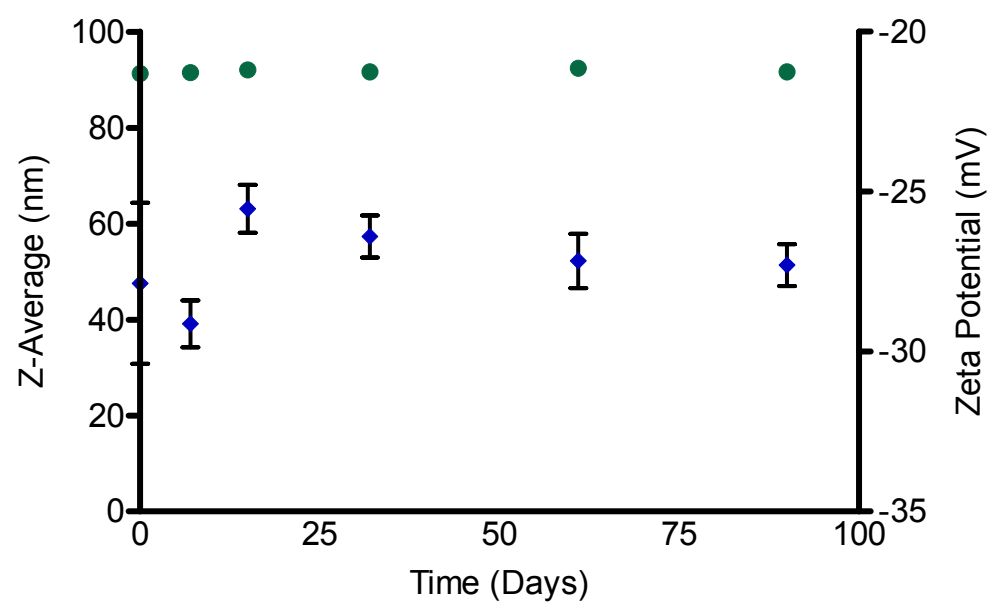

Figure 5-1. Stability data for Pac-RGD-TLCL dispersion

Notes. The liposomes were stored at $5 \pm 3^{\circ} \mathrm{C}$ in $2 \mathrm{cc}$ amber vials. Samples were withdrawn at specific intervals and analyzed for paclitaxel retention, PSD, and zeta potential. A. For the determination of paclitaxel retention, the samples were subjected to minicolumn centrifugation in Sephadex G-25 macro spin columns to separate free paclitaxel from total paclitaxel. The eluate containing entrapped paclitaxel was digested with ethanol, and quantified by RPHPLC method. The results indicate the mean \pm standard deviation for three injections ( $\Delta$ Paclitaxel concentration; - Percent paclitaxel retained in the liposome). B. PSD and zeta potential was determined in samples suitably diluted with HPLC grade water. The results indicate mean \pm standard deviation of three independent measurements (•PSD; $\bullet$ Zeta potential). 


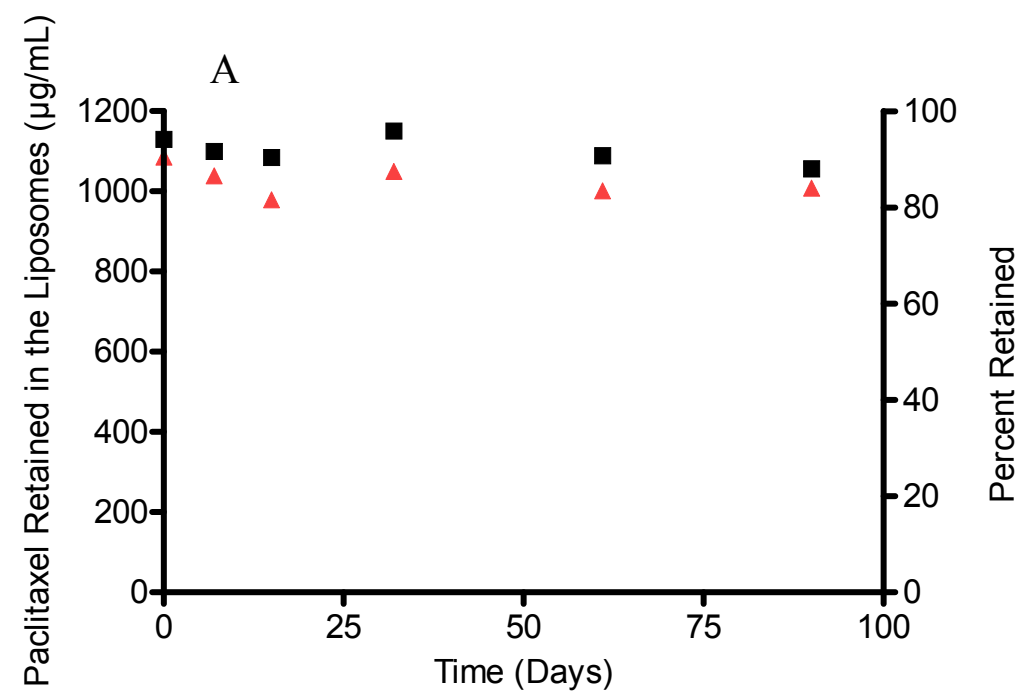

B

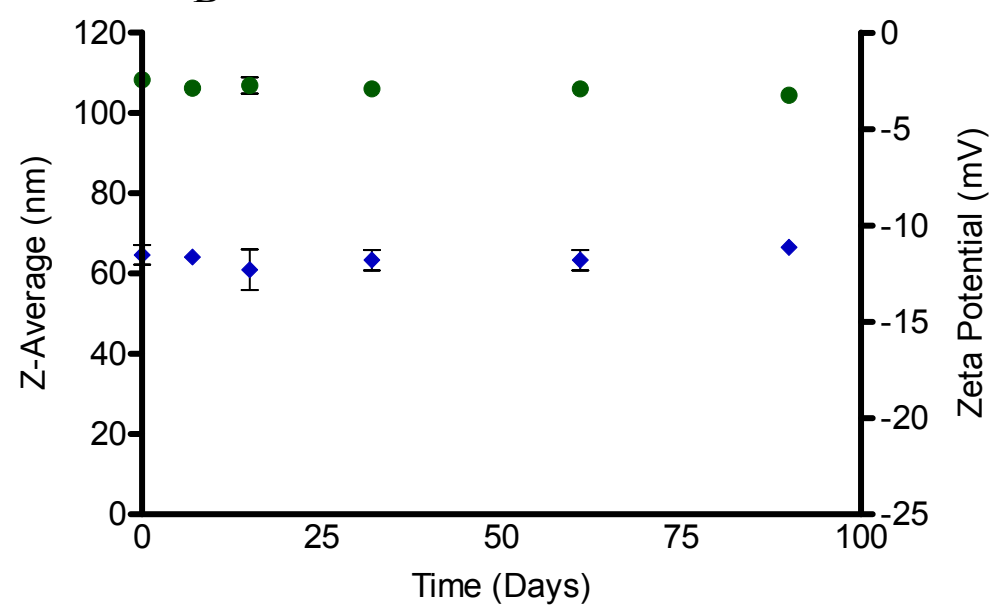

Figure 5-2. Stability data for Pac-Tf-TLCL dispersion

Notes. The liposomes were stored at $5 \pm 3^{\circ} \mathrm{C}$ in $2 \mathrm{cc}$ amber vials. Samples were withdrawn at specific intervals and analyzed for paclitaxel retention, PSD, and zeta potential. A. For the determination of paclitaxel retention, the samples were subjected to minicolumn centrifugation in Sephadex G-25 macro spin columns to separate free paclitaxel from total paclitaxel. The eluate containing entrapped paclitaxel was digested with ethanol, and quantified by RPHPLC method. The results indicate the mean \pm standard deviation for three injections ( $\Delta$ Paclitaxel concentration; $\boldsymbol{\square}$ Percent paclitaxel retained in the liposome). B. PSD and zeta potential was determined in samples suitably diluted with HPLC grade water. The results indicate mean \pm standard deviation of three independent measurements (• PSD; Zeta potential). 


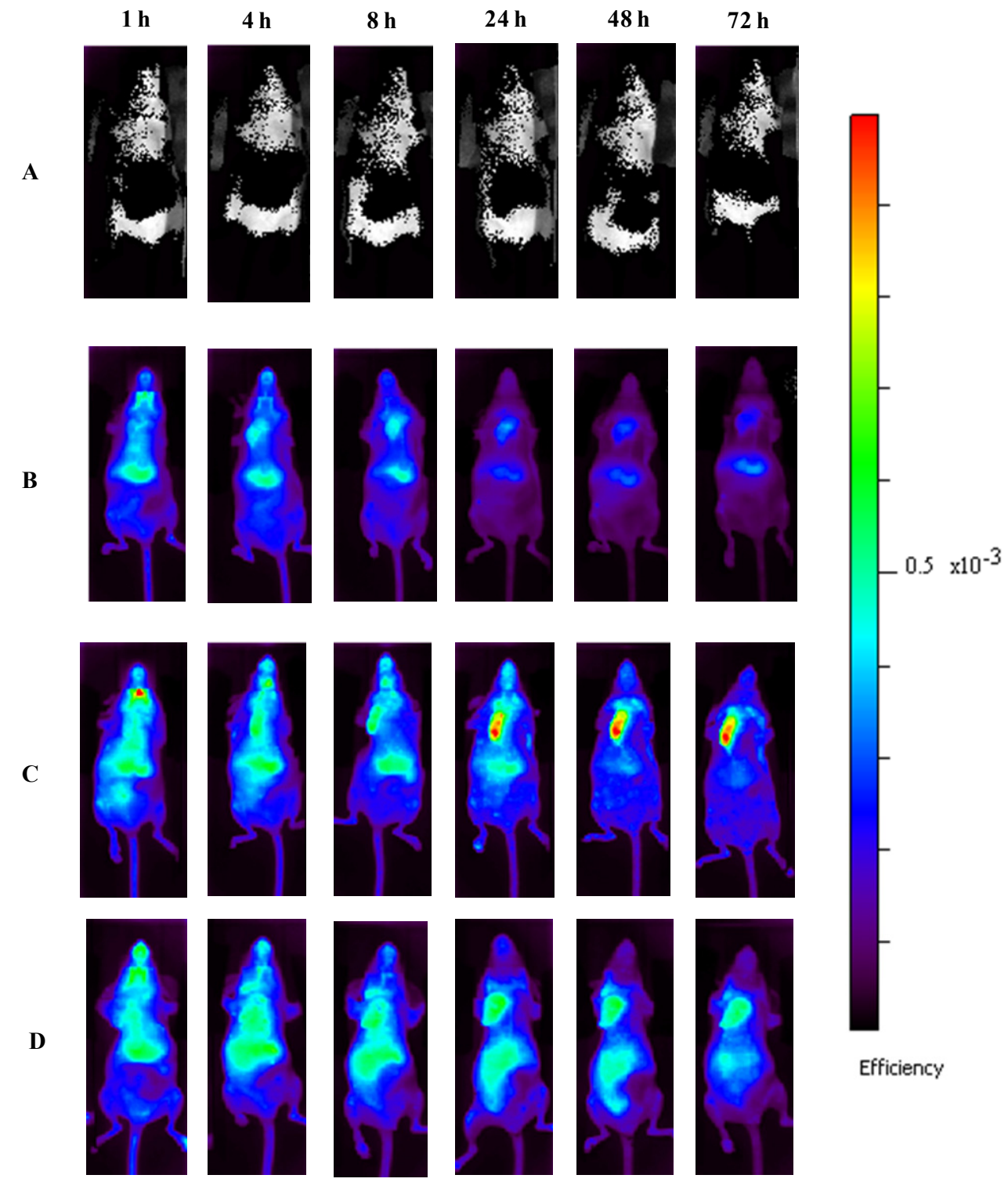

Figure 5-3. Representative NIRF images of localization of DiR formulations in mice bearing MDA-MB-231 breast cancer

Notes. MDA-MB-231 cells were inoculated subcutaneously into the breast tissue in the axillary region of female athymic nude mice. The animals received $50 \mu \mathrm{L}$ of normal saline (A), $5 \mu \mathrm{g}$ of DiR in $\sim 50 \mu \mathrm{L}$ of micellar solution formulation (B), or LCL formulation (C) or RGD-TLCL formulation (D). NIRF images were acquired at 1, 4, 8, 24,48 , and 72 hours using Xenogen IVIS ${ }^{\circledR}$ Lumina imaging system with excitation and emission wavelengths of $745 \mathrm{~nm}$ and $780 \mathrm{~nm}$ respectively. The images from representative animal in each treatment group are presented. 
The liposome formulations showed gradual increase in accumulation in the tumors and reached the maximum intensity in $24-48$ hours. DiR fluorescent intensity from the liposomal formulations in the tumor tissue was observed up to $72 \mathrm{~h}$ and was higher compared to DiR solution formulation. DiR fluorescence intensity was also higher in the abdominal region of the mice which received liposomal formulations. This could be due to clearance of the liposomal particles by the Kupffer cells of liver and fixed macrophages of spleen. The uptake in the liver and spleen for DiR-RGD-TLCL was higher compared to DiR-LCL or DiR solution formulation and was more clearly evident in the images acquired with isolated tissues (Figure 5-4).

The fluorescence intensity of DiR was calculated in tumor tissue and normal tissue selected as the region of interest. These ROI measurement results can be expressed in terms of photon emission from the subject or radiance. The radiance unit of photons $/ \mathrm{sec} / \mathrm{cm} 2 / \mathrm{sr}$ is the number of photons per second that leave a square centimeter of tissue and radiate into a solid angle of one steradian (sr). The measurement of units of radiance takes into account measurement settings like F/stop, binning, and field of view. The images acquired in the same session with the same animal with different camera settings will produce same signal amplitude because the photon emission of the animal remains the same. On the other hand, the fluorescent signal detected from the animal depends on the amount of fluorophore present in the animal and the intensity of the incident excitation light. But, the excitation light incident on the animal stage is not uniform over the field of view. The amount of fluorophore being constant, the fluorescence emission image needs to be normalized to incident excitation intensity. The user manual for Living Image ${ }^{\circledR} 3.2$ software (Caliper Life Sciences, Hopkinton, MA) suggests that calculating fluorescent image data in terms of efficiency (Efficiency $=$ Radiance of the subject/Illumination intensity) can eliminate the variable excitation light from the measurement and enable a more quantitative comparison of fluorescent signals. Normalizing all the variables in the measurement is important for the kinetic measurements because the amount of DiR remaining in the animal can vary with time which can be quantitatively monitored even if the measurements were made at different camera settings, and differing intensity of light.

The total efficiency data with tumor selected as the region of interest is presented in Figure 5-5. The total efficiency for the solution formulation increased steadily up to $8 \mathrm{~h}$ and then decreased until $72 \mathrm{~h}$. The DiR-LCL formulation showed a biphasic distribution with an increase in the initial $4 \mathrm{~h}$ of administration, a decrease from $4-8 \mathrm{~h}$, and then a steady increase to a maximum in $48 \mathrm{~h}$. The total efficiency in the tumor was maintained for $72 \mathrm{~h}$. The DiR-RGD-TLCL formulation also showed similar kinetics but the total efficiency was lower compared to DiR-LCL. Bonferroni post test analysis of two-way ANOVA showed that all the DiR containing formulations were statistically significant ( $p$ $<0.01$ ) from normal saline control. At $48 \mathrm{~h}$ time point, the total efficiency of DiR-LCL was significantly higher $(p<0.001)$ than DiR-RGD-TLCL, and DiR micellar solution formulation. But, the total efficiency of DiR-RGD-TLCL was not significantly different from DiR micellar solution formulation. The total efficiency in the flank region to represent the concentration of DiR in the circulation is presented in Figure 5-6. The total efficiency in the normal tissue decreased with time. The normal tissue accumulation 


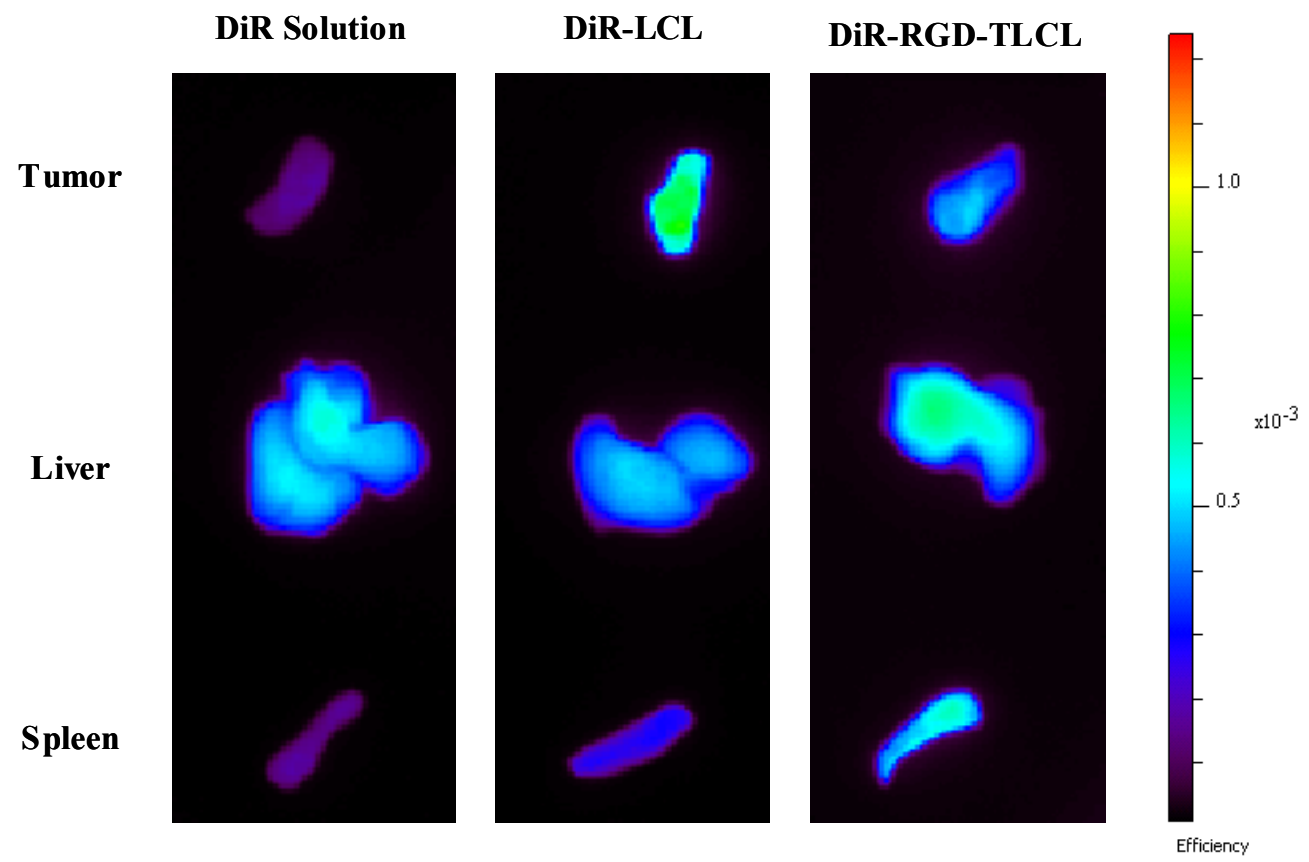

Figure 5-4. NIRF images of tissues isolated $72 \mathrm{~h}$ post injection showing the accumulation of DiR in tumor, liver, and spleen

Notes. MDA-MB-231 cells were inoculated subcutaneously into the breast tissue in the axillary region of female athymic nude mice. The animals received $5 \mu \mathrm{g}$ of $\mathrm{DiR}$ in $50 \mu \mathrm{L}$ of micellar solution formulation or LCL formulation or RGD-TLCL formulation. The animals were sacrificed after 72 hours and the tissues were isolated. NIRF images were acquired using Xenogen IVIS ${ }^{\circledR}$ Lumina imaging system with excitation and emission wavelengths of $745 \mathrm{~nm}$ and $780 \mathrm{~nm}$ respectively. The representative images of tissues from the animals shown in Figure 5-3 for each of the DiR treatment group are shown. 


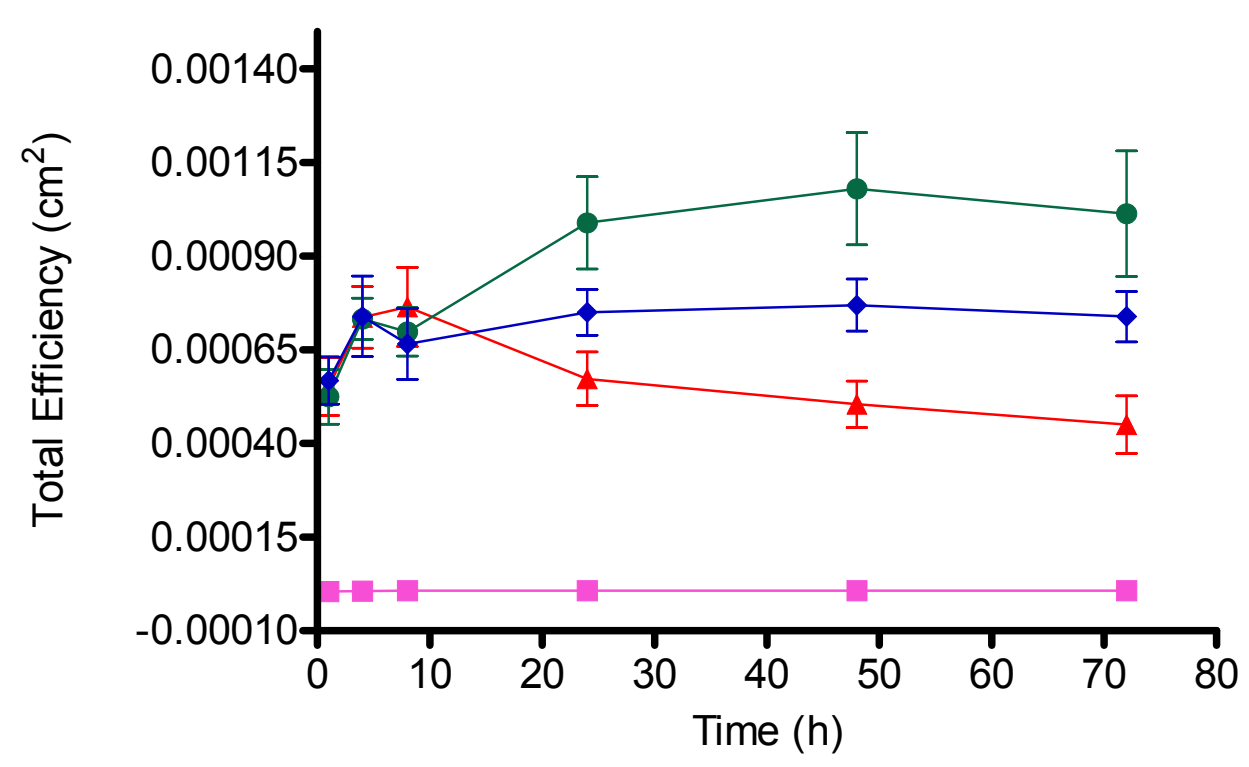

Figure 5-5. Localization of DiR-loaded liposomal formulations in tumor tissue calculated from NIRF imaging

Notes. MDA-MB-231 cells were inoculated subcutaneously into the breast tissue in the axillary region of female athymic nude mice. The animals received $50 \mu \mathrm{L}$ of normal saline (- $), 5 \mu \mathrm{g}$ of DiR in $\sim 50 \mu \mathrm{L}$ of micellar solution formulation ( $\triangle-)$, or LCL formulation ( $\bullet$ ) or RGD-TLCL formulation ( $\star$ ). NIRF images were acquired at $1,4,8$, 24,48 , and 72 hours using Xenogen IVIS ${ }^{\circledR}$ Lumina imaging system with excitation and emission wavelengths of $745 \mathrm{~nm}$ and $780 \mathrm{~nm}$ respectively. The total efficiency was calculated in tumor selected as region of interest. The results indicate mean $\pm \mathrm{SEM}$ of ROI measurements from five animals. 


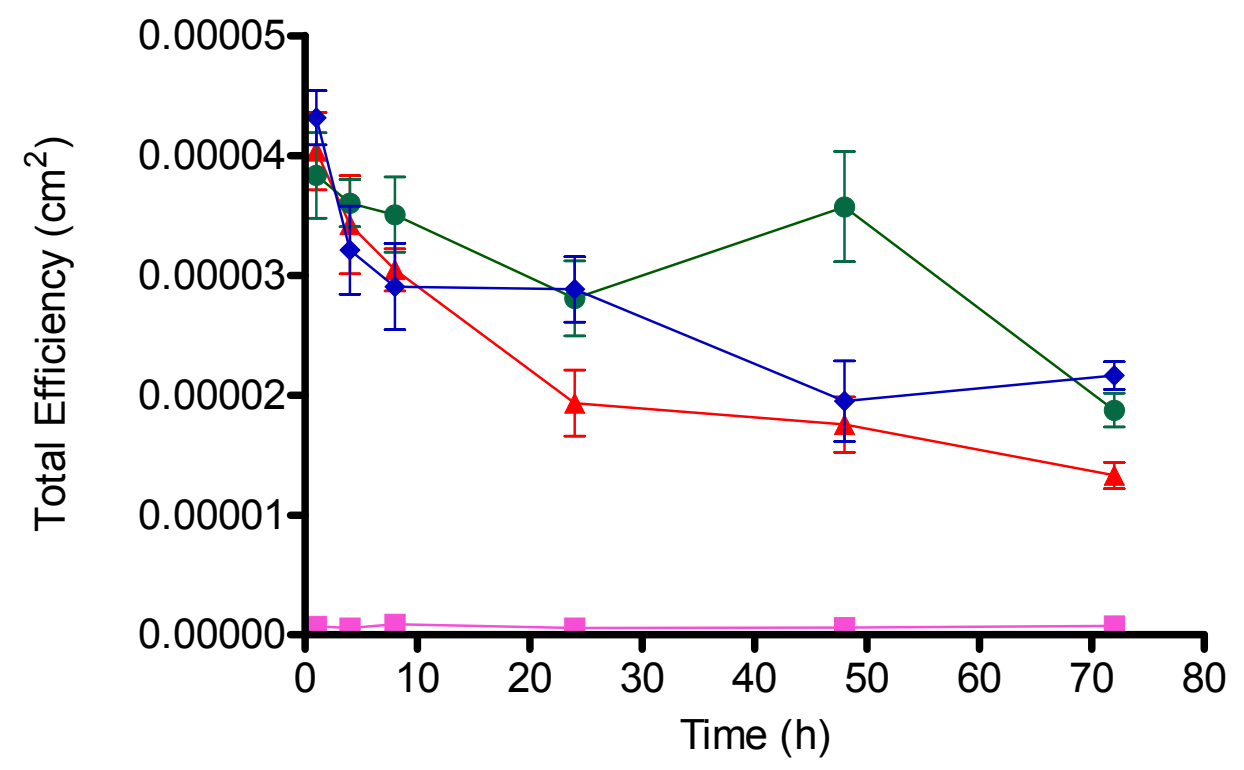

Figure 5-6. Localization of DiR formulations in the flank region to represent the concentration in the circulation

Notes. MDA-MB-231 cells were inoculated subcutaneously into the breast tissue in the axillary region of female athymic nude mice. The animals received $50 \mu \mathrm{L}$ of normal saline (- $), 5 \mu \mathrm{g}$ of DiR in $\sim 50 \mu \mathrm{L}$ of micellar solution formulation $(-)$ ), or LCL formulation ( $\bullet$ ) or RGD-TLCL formulation ( $\star$ ). NIRF images were acquired at $1,4,8$, 24,48 , and 72 hours using Xenogen IVIS ${ }^{\circledR}$ Lumina imaging system with excitation and emission wavelengths of $745 \mathrm{~nm}$ and $780 \mathrm{~nm}$ respectively. The total efficiency was calculated in flank region to represent normal tissue and the concentration in circulation. The results indicate mean \pm SEM of ROI measurements from five animals. 
followed a similar trend as tumor accumulation with lower levels of DiR micellar solution formulations from 24-48 hours. DiR-LCL showed higher normal tissue levels than DiR-RGD-TLCL at $48 \mathrm{~h}$.

The targeting with cRGD peptide is expected to improve the accumulation of the liposomes due to receptor mediated endocytosis. But, the tumor accumulation of DiRRGD-TLCL was lower than DiR-LCL in this animal model. The size and charge of these two liposomes were similar and should exhibit similar longer circulation properties. Thus, the accumulation of Dir-RGD-TLCL should be at least similar to DiR-LCL because of passive accumulation of the liposomes at the tumor due to EPR effect. In contrast, the presence of RGD peptide on the surface of these targeted liposomes increased opsonization and macrophage uptake as shown by their increased accumulation in the liver and spleen. The total efficiency in the peritoneal cavity comprised of liver and spleen which are the major organs of clearance of liposomes was higher for DiR-RGDTLCL compared to DiR-LCL and DiR solution formulation (Figure 5-7). The accumulation of DiR-RGD-TLCL in the peritoneal cavity was significantly higher $(p<$ 0.001 ) than DiR-LCL at $1,24,48$, and $72 \mathrm{~h}$ imaging time points. Their accumulation was also significantly higher $(p<0.001)$ than DiR solution formulation at all imaging time points studied. The DiR-LCL formulation also showed higher accumulation than DiR solution formulation at $8 \mathrm{~h}(\mathrm{p}<0.05), 24 \mathrm{~h}(\mathrm{p}<0.001)$, and $48 \mathrm{~h}(\mathrm{p}<0.01)$ imaging time points. The higher clearance of targeted liposomes resulted in lower blood levels as shown by the measurements from normal tissue which in turn lead to a reduced accumulation in the tumors. The results were similar to literature report measuring the concentration of radio labeled RGD conjugated liposomes in murine $\mathrm{C} 26$ colon carcinoma model [166]. The RGD conjugated liposomes showed lower blood levels, and an increased accumulation in spleen and liver compared to non conjugated liposomes. The expression of $\alpha_{v} \beta_{3}$ integrin receptors by the mononuclear cells in the spleen was suggested as a mechanism of splenic uptake of these liposomes by phagocytosis. The lower accumulation in the tumor could also be due to the limited effect on tumors with pre-established vasculature because integrins are not expressed in the interior parts of the tumor.

The total efficiency of tumor tissue was compared to the total efficiency on normal tissue calculated from the flank as the region of interest. The tumor/normal tissue $(\mathrm{T} / \mathrm{N})$ ratio was calculated as a measure of tumor targeting index and is presented in Figure 5-8. The $\mathrm{T} / \mathrm{N}$ ratio increased for all the DiR containing formulations to more than 20 within 8 $\mathrm{h}$ of dosing and continued to increase for all the formulations. Even though DiR-LCL showed higher accumulation in the tumor compared to DiR-RGD-TLCL, and DiR solution formulation, there was no significant difference was observed in the $\mathrm{T} / \mathrm{N}$ ratio of these DiR formulations. It can be explained based on the fact the total efficiency decreases in the normal tissue with time due to clearance. The decrease is greater for the formulations cleared rapidly from the circulation. Since $\mathrm{T} / \mathrm{N}$ ratio takes the difference in total efficiency between tumor and normal tissue in to the calculation, the absolute difference at the extent of accumulation is not taken in to account. Since T/N ratio is a relative measure showing the extent of accumulation in the tumor versus normal tissue, 


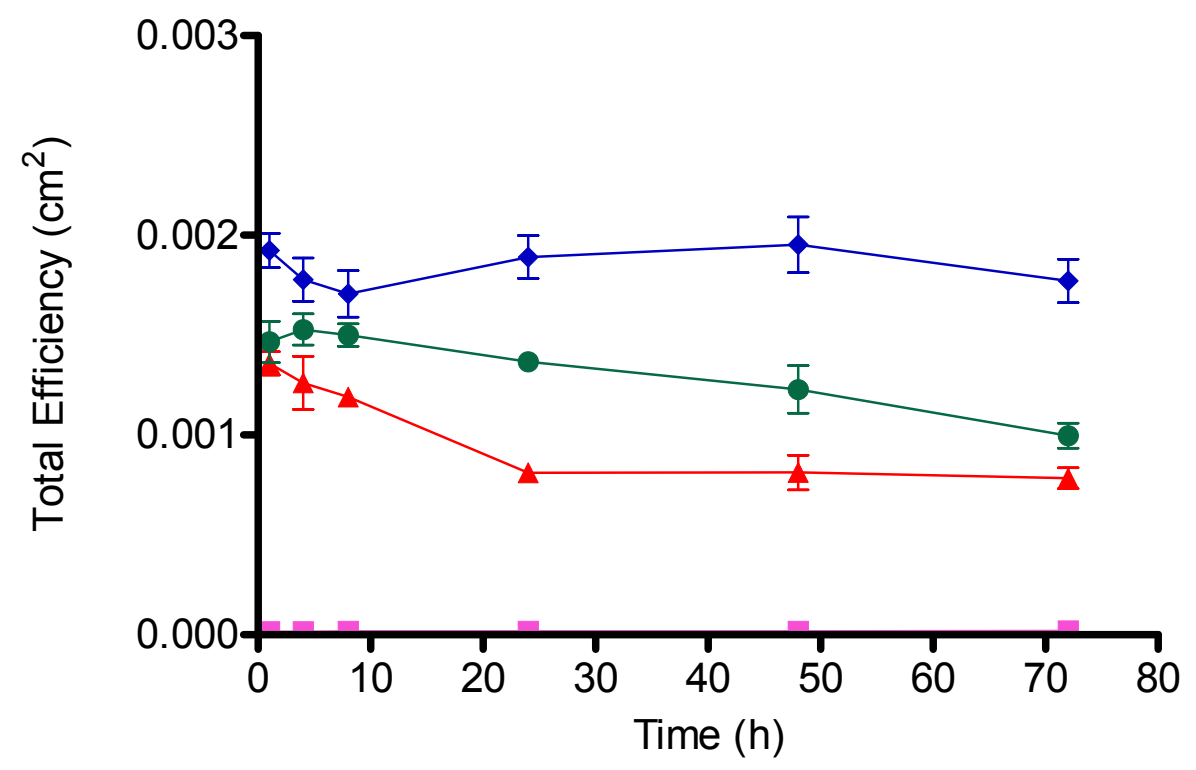

Figure 5-7. Localization of DiR formulations in peritoneal cavity to represent uptake in liver and spleen

Notes. MDA-MB-231 cells were inoculated subcutaneously into the breast tissue in the axillary region of female athymic nude mice. The animals received $50 \mu \mathrm{L}$ of normal saline (- $), 5 \mu \mathrm{g}$ of DiR in $\sim 50 \mu \mathrm{L}$ of micellar solution formulation $(-)$ ), or LCL formulation ( $\bullet$ ) or RGD-TLCL formulation ( $\star$ ). NIRF images were acquired at $1,4,8$, 24,48 , and 72 hours using Xenogen IVIS ${ }^{\circledR}$ Lumina imaging system with excitation and emission wavelengths of $745 \mathrm{~nm}$ and $780 \mathrm{~nm}$ respectively. The total efficiency was calculated in peritoneal cavity to represent the uptake in liver and spleen. The results indicate mean $\pm \mathrm{SEM}$ of ROI measurements from five animals. 


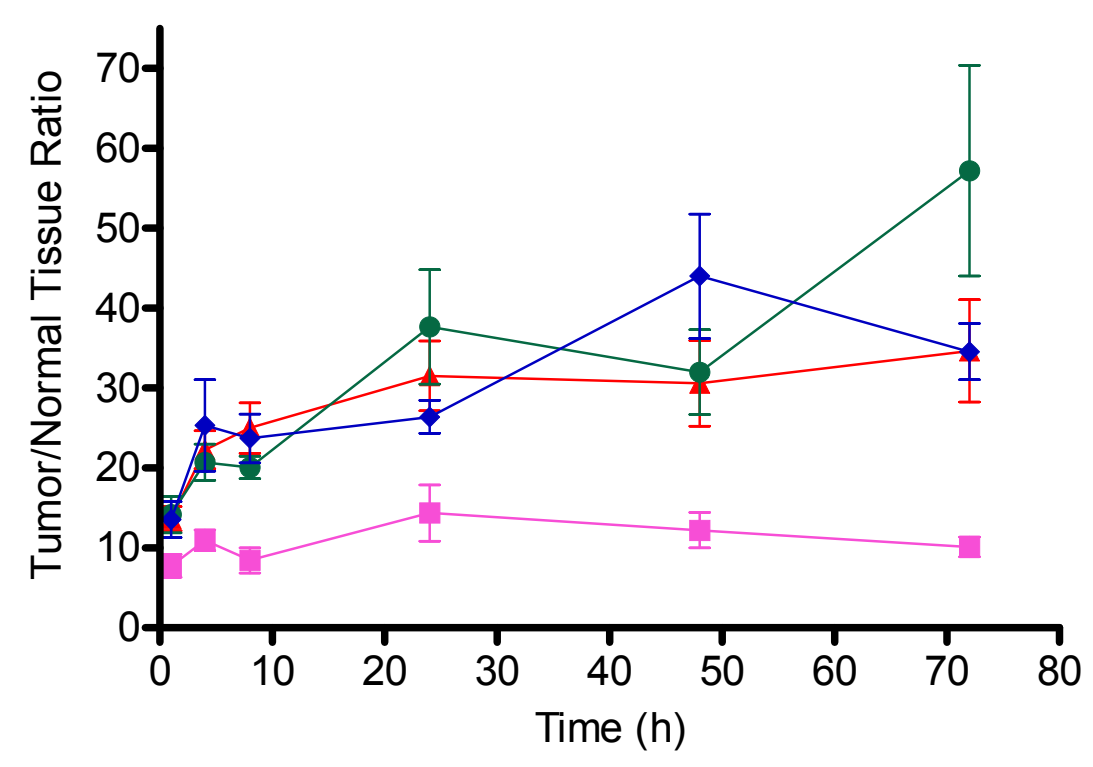

Figure 5-8. Tumor/normal tissue ratio of DiR formulations calculated from NIRF imaging

Notes. MDA-MB-231 cells were inoculated subcutaneously into the breast tissue in the axillary region of female athymic nude mice. The animals received $50 \mu \mathrm{L}$ of normal

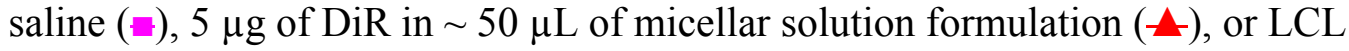
formulation ( $\bullet$ or RGD-TLCL formulation ( $\bullet$ ). NIRF images were acquired at $1,4,8$, 24,48 , and 72 hours using Xenogen IVIS ${ }^{\circledR}$ Lumina imaging system with excitation and emission wavelengths of $745 \mathrm{~nm}$ and $780 \mathrm{~nm}$ respectively. The tumor/normal tissue ratio was calculated from the ROI selected for tumor and flank tissue respectively. The results indicate mean $\pm \mathrm{SEM}$ of ROI measurements from five animals. 
it can only be used as a guide, and the absolute total efficiency needs to be considered for comparison between the formulations.

\subsubsection{Quantification of Paclitaxel from Plasma and Tumor Tissues to Determine Biodistribution of Paclitaxel-Loaded Liposomes}

The biodistribution was evaluated for paclitaxel micellar solution formulation, PacLCL and Pac-RGD-TLCL in mouse xenografts bearing MDA-MB-231 breast cancer by quantifying paclitaxel in plasma and tumor. The size of the liposomal formulation was also varied to estimate the effect of size on plasma and tumor levels. The results are presented in Figure 5-9. Paclitaxel levels in plasma $24 \mathrm{~h}$ after dosing shows that there was no significant difference between paclitaxel solution formulation and the liposomal formulations of size $\sim 160 \mathrm{~nm}$. But, the liposomes of size $\sim 80 \mathrm{~nm}$ showed significantly higher $(p<0.001)$ plasma levels compared to solution formulation and liposomes of size $\sim 160 \mathrm{~nm}$. The tumor accumulation of $\sim 160 \mathrm{~nm}$ liposomes was less than solution formulation at 24 hours post injection. But the Pac-RGD-TLCL showed significantly higher accumulation $(p<0.001)$ in the tumor compared to paclitaxel solution formulation, and Pac-LCL and Pac-RGD-TLCL of $\sim 160 \mathrm{~nm}$ diameter. These results indicate that the effect of conjugation with RGD was not perceivable when the liposome size was $\sim 160 \mathrm{~nm}$. However, a significant effect of size was observed in plasma levels as well as tumor accumulation levels for paclitaxel from liposomes when the size was reduced to $\sim 80 \mathrm{~nm}$. The results were consistent with NIRF imaging results with similar blood levels and tumor accumulation for non-targeted and targeted liposomes at 24 hours post injection.

\subsubsection{Antitumor Efficacy of Tumor Vasculature and Tumor Cell Targeted Liposomes}

The antitumor efficacy of paclitaxel formulations was evaluated in mouse xenografts bearing MDA-MB-231 breast cancer in the axillary region. Tumor vasculature targeted RGD conjugated liposomes did not show better accumulation than non-targeted liposomes in the biodistribution studies. So, another group of animals was included in the antitumor efficacy study to compare the efficacy of these tumor vasculature targeted liposomes with tumor cell targeted liposomes. Since TfR1 receptors are expressed by the tumor cells, targeting with transferrin attached to the surface of paclitaxel-loaded liposomes is expected to improve the efficacy of the treatment by receptor mediated endocytosis of the tumor cells. Figure 5-10 shows the effect of different formulations on tumor volumes measured twice weekly for six weeks. The statistical analysis was performed using two-way ANOVA with data up to 39 days post tumor inoculation because all the animals in normal saline control group reached euthanasia criteria of tumor size greater than $2 \mathrm{~cm}$ in diameter, and were sacrificed. Bonferroni post test comparisons between treatment groups showed that all the paclitaxel treatment groups significantly $(p<0.001)$ reduced tumor volume at 39 days post tumor inoculation compared to normal saline control group. The tumor cell targeted Pac-Tf-TLCL showed 

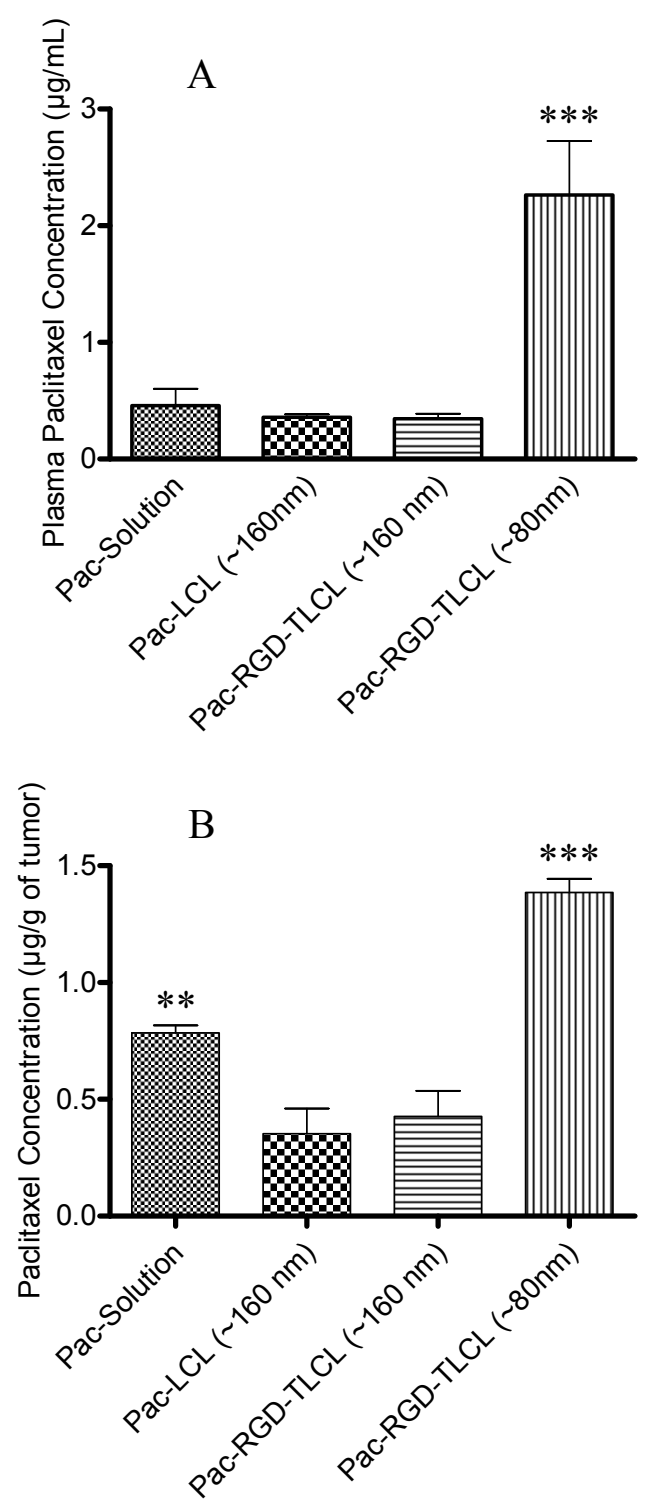

Figure 5-9. Biodistribution of paclitaxel formulations in plasma (A), and tumor (B) of mouse xenografts bearing MDA-MB-231 breast cancer

Notes. MDA-MB-231 cells were inoculated subcutaneously into the breast tissue in the axillary region of female athymic nude mice. The animals received $7.5 \mathrm{mg} / \mathrm{kg}$ of paclitaxel in solution formulation or Pac-LCL, $160 \mathrm{~nm}$ or Pac-RGD-TLCL, $160 \mathrm{~nm}$ or Pac-RGD-TLCL, $80 \mathrm{~nm}$. Blood samples were collected after 24 hours of dosing. The animals were sacrificed and tumor tissues were collected. The concentration of paclitaxel in plasma and tumor tissues was determined by the RPHPLC. The results indicate mean \pm standard deviation of samples from three animals. In A, *** is $p<0.001$ for Pac-RGDTLCL $(\sim 80 \mathrm{~nm})$ versus all other formulations. In $\mathrm{B}, * *$ is $p<0.01$ for Pac-Solution formulation versus Pac-LCL and Pac-RGD-TLCL of size $\sim 160 \mathrm{~nm}$, and $* * *$ is $p<0.001$ for Pac-RGD-TLCL $(\sim 80 \mathrm{~nm})$ versus all the other formulations. 


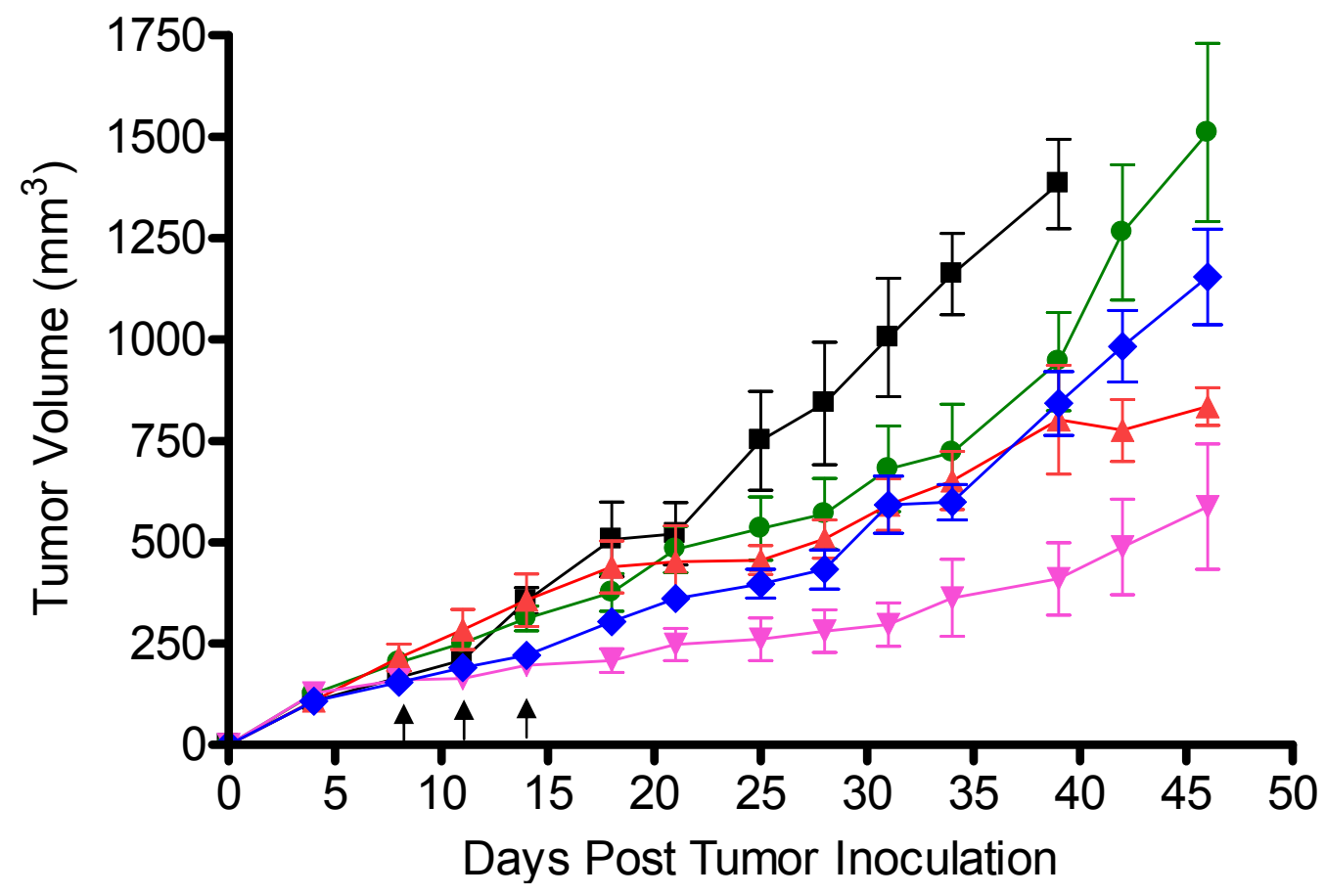

Figure 5-10. Antitumor efficacy of paclitaxel formulations in mouse xenografts bearing MDA-MB-231 breast cancer

Notes. MDA-MB-231 cells were inoculated subcutaneously into the breast tissue in the axillary region of female athymic nude mice. The animals received $100 \mu \mathrm{L}$ of normal saline as control (-) or $7.5 \mathrm{mg} / \mathrm{kg}$ of paclitaxel in solution formulation (A) or Pac-LCL $(\bullet)$, or Pac-RGD-TLCL ( $)$ or Pac-Tf-TLCL $(\forall)$ on 8, 11, and 14 days post tumor inoculation (indicated by arrows). The tumor diameters were measured twice weekly and the tumor volume was calculated. The results indicate mean $\pm \mathrm{SEM}$ of results calculated from five animals. 
highest efficacy in tumor regression and was significantly $(p<0.001)$ higher than paclitaxel solution formulation, Pac-LCL, and Pac-RGD-TLCL. In contrast, the tumor vasculature targeted Pac-RGD-TLCL did not show a significant reduction in tumor volume compared to paclitaxel solution formulation, and Pac-LCL. The observations in the antitumor efficacy study for Pac-RGD-TLCL was similar to the observations from the biodistribution studies with NIRF imaging and quantification of paclitaxel from cRGD conjugated liposomes. The tumor vasculature targeting with cRGD should have increased the localization followed by uptake of paclitaxel by receptor mediated endocytosis. But the overall efficacy was similar to non-targeted liposomes. The possible reasons for this could be due to higher clearance of cRGD conjugated liposomes by an additional mechanism of phagocytosis followed by splenic uptake due to their binding to $\alpha_{v} \beta_{3}$ integrin receptors expressed by the mononuclear cells in the spleen. So the total number of liposomes available for binding to the $\alpha_{\mathrm{v}} \beta_{3}$ integrin receptors expressed in the tumor vasculature is reduced. Apart from that, well established tumors express $\alpha_{\mathrm{v}} \beta_{3}$ integrin receptors only on the subset of vessels associated with tumor margin [174]. So, apoptosis is induced only to those tumor cells near that margin. Even though, the $\alpha_{v} \beta_{3}$ integrin receptors expressed on the tumor vasculature are readily accessible by the liposomes, the expression of these receptors is limited only to neovasculature and plays a major limiting factor in treating well established tumors. Nevertheless, Pac-RGD-TLCL would prove to be an effective strategy for treating metastatic tumors and primary tumors at very early stages of development. In contrast to tumor vasculature targeted liposomes, the tumor cell targeted Pac-Tf-TLCL was very effective in minimizing the tumor growth rate of breast cancer in mouse xenografts. The density of TfR1 on the surface of cells is correlated with the extent of cell growth and division, and consequently, the tumor cells express more TfR1 than normal cells. In contrast to tumor vasculature targeted liposomes, the targeting approach with transferrin to target tumor cells takes advantage of the active process of $\mathrm{Fe}$ transport to deliver paclitaxel to the tumor cells. Even though the rate limiting step is the accumulation of these liposomes in the tumor sites by passive accumulation due to EPR effect, the receptor mediated endocytosis by the cancer cells through transferrin is more efficient and hence improved efficacy is observed.

\subsection{SUMMARY AND CONCLUSIONS}

The biodistribution of cRGD conjugated paclitaxel-loaded liposomes was evaluated in mouse xenografts bearing MDA-MB-231 breast cancer by non-invasive NIRF imaging method. The accumulation of DiR-RGD-TLCL in the tumor was comparable to nontargeted LCL. It was less than DiR-LCL at $48 \mathrm{~h}$ time point of imaging. The possible reason is the clearance of these targeted liposomes by splenic uptake due to expression of integrin receptors by mononuclear cells in spleen. Higher fluorescence intensity was observed in the abdominal region of the animals in the liposomal formulation groups suggesting the involvement of liver and spleen in clearance. The paclitaxel quantification from liposomal formulations also showed a similar trend with paclitaxel-loaded cRGD conjugated liposomes and non-targeted liposomes showing comparable accumulation in the tumors. The effect of size of the liposomes was evident with smaller sized liposomes

of size $\sim 80 \mathrm{~nm}$ showing higher blood levels, and tumor tissue levels compared to 
liposomes of approximately double the size. These smaller liposomes also showed significantly higher blood levels and tumor tissue levels compared to paclitaxel solution formulation. The antitumor efficacy study in breast cancer mouse xenograft model showed that tumor cell targeted transferrin conjugated paclitaxel-loaded liposomes have higher efficacy in minimizing the tumor growth compared to tumor vasculature targeted cRGD conjugated paclitaxel-loaded liposomes. The Pac-Tf-TLCL showed higher efficacy than paclitaxel solution formulation suggesting the treatment with this formulation is superior to the currently approved therapy using Taxol ${ }^{\circledR}$. Tumor vasculature targeting did not produce an appreciable effect compared to non-targeted liposomes possibly due to limited expression of integrin receptors in the core of well established tumors and due to splenic uptake of the liposomes via integrin receptors expressed by mononuclear cells. Even with the enhanced uptake by the spleen, the cRGD conjugated liposomes produced comparable effect produced by non-targeted liposomes, and paclitaxel solution formulation suggesting that these liposomes have better therapeutic potential in cases of metastasis and primary tumors with fast developing vasculature wherein the expression of $\alpha_{v} \beta_{3}$ integrin receptors is amplified. 


\section{CHAPTER 6. LYOPHILIZATION OF PACLITAXEL-LOADED LIPOSOMES TO IMPROVE LONG TERM STABILITY}

Paclitaxel is one of the most effective chemotherapeutic agents used in the treatment of solid tumors. It is a natural product obtained from the bark of Pacific Yew tree Taxus brevifolia $[8,9]$. Paclitaxel, classified under Class IV of Biopharmaceutic Classification System (BCS) proposed by Amidon, has poor solubility and permeability [271, 272]. Due to its poor aqueous solubility, Taxol ${ }^{\circledR}$ (Bristol-Myers Squibb Company, Princeton, NJ), one of the marketed formulation of paclitaxel was formulated with Cremophor ${ }^{\mathbb{R}} \mathrm{EL}$ and ethanol. Although the formulation is diluted before use, the concentration of Cremophor ${ }^{\circledR} \mathrm{EL}$ is high enough to cause serious hypersensitivity reactions on parenteral administration, and require pretreatment with antihistamines and corticosteroids [15]. In addition, the use of free paclitaxel can affect normal tissues and result in systemic toxicities such as myelosuppression, and peripheral neuropathy [17]. So, newer drug delivery systems are sought so that paclitaxel can be delivered locally to the tumor sites without affecting normal tissues. Liposomes are biocompatible carriers with ability to incorporate both lipophilic and hydrophilic drugs [23]. Encapsulating drugs within vesicles has the potential advantage of higher drug carrying capacity compared to attaching them to single polymer chain [250]. Preparation of liposomes of paclitaxel can alter the pharmacokinetic and pharmacodynamic properties of the free drug and result in reduced toxicity and enhanced efficacy of the treatment [251].

Stability of the liposomes in dispersion during storage is a major concern in the development of liposomal formulations. Phospholipids from the liposomes can undergo hydrolysis and/or oxidation and result in physical degradation when stored as aqueous dispersions. As a result, the encapsulated drug tends to leak from the vesicles. Apart from leakage, the liposomes can aggregate or fuse together resulting in an increase in particle size distribution. The loss of encapsulated drug, and increase in vesicle size during storage can affect long-term stability of the product and hinder the drug development process. The stability of liposomes can be improved by freezing, lyophilization, spray drying, and supercritical fluid technology [29-31]. Lyophilization is one of the preferred method to improve long-term stability of liposomes especially those containing thermosensitive drugs [32]. It can prevent hydrolysis of phospholipids, and physical degradation during storage of the liposomes [34]. By removing water from the liposomal dispersion by lyophilization, the structural and permeability properties can be retained [33]. Interaction of water with hydrophilic phosphate head groups of phospholipids plays a key role in the formation of liposomes and hence, removal of water presents more challenges. In addition, the lyophilization process itself can induce physical changes such as loss of the encapsulated agent, and an increase in vesicle size [34]. Therefore, careful selection and optimization of the formulation and process is necessary to improve the long-term stability of paclitaxel-loaded liposomes.

The ability of several carbohydrates to protect liposomes during lyophilization is described by 'water replacement' hypothesis [208-210] and 'vitrification' hypothesis [211-213]. This capability to protect liposomes during dehydration originated from the 
fact that organisms such as brine shrimp cysts and some species of nematodes survive dehydration which is related to their capacity to produce nonreducing disaccharide trehalose $[214,215,221]$. These anhydrobiotic organisms survive for several years in dry state and return to normal metabolic activities on rehydration. Madden et al used this analogy to protect the integrity of liposomes with trehalose and other sugars [33]. Next to trehalose sucrose is widely used as a lyoprotectant in the development of lyophilized liposomal formulations [249, 260, 273]. It is a non-crystallizing solute and remains in the amorphous state during the lyophilization process. In this research work, we evaluated the effect of sucrose as a lyoprotectant in the development of paclitaxel-loaded longcirculating liposomes (Pac-LCL). The effect of lipid-sugar ratio, and internal-external ratio with sucrose was evaluated for Pac-LCL. The optimized composition was used for the lyophilization of cRGD or transferrin conjugated targeted long-circulating liposomes. The shelf-life of these formulations was evaluated for three months.

\subsection{MATERIALS AND METHODS}

\subsubsection{Materials}

Paclitaxel was procured from 21CEC Pharmaceuticals (Bradenton, FL). The phospholipids used in this work were all of research grade. Egg phosphatidylcholine (EPC), hydrogenated soy phosphatidylcholine (HSPC) and 1,2-distearoyl-sn-glycero-3phoshoethanolamine-N-(poly(ethylene glycol)2000) (DSPE-PEG ${ }_{2000}$ ) were obtained from Northern Lipids (Burnbay, BC, Canada). 1,2-distearoyl-sn-glycero-3phoshoethanolamine-N-(maleimide poly(ethylene glycol)2000) (maleimide DSPE$\mathrm{PEG}_{2000}$ ) and 1,2-distearoyl-sn-glycero-3-phoshoethanolamine-N-(biotinyl poly(ethylene glycol)2000) (biotinyl DSPE-PEG 2000 ) were obtained from Avanti Polar Lipids (Alabaster, AL). Cyclo (Arg-Gly-Asp-D-Phe-Cys) peptide (cRGD) was obtained from Peptides International (Louisville, KY). Biotin labeled human transferrin (Tf), cholesterol, $\alpha$-tocopherol, and sucrose were purchased from Sigma (St. Louis, MO). Streptavidin was obtained from PROzyme (Hayward, CA). HPLC grade water, acetonitrile, and methanol were obtained from Fisher Scientific (Fair Lawn, NJ). HPLC grade chloroform was purchased from Acros Organics (Morris Plains, NJ). All other chemicals and reagents were of analytical grade and used without further purification or characterization.

\subsubsection{Preparation of Paclitaxel-Loaded Long-Circulating Liposomes (Pac-LCL)}

The Pac-LCL was be prepared by the lipid film hydration-extrusion method using EPC, HSPC, DSPE-PEG 2000 , and cholesterol. The lipid film was prepared by dissolving

paclitaxel, phospholipids, cholesterol, and $\alpha$-tocopherol in 9:1 chloroform-methanol. The solution obtained was evaporated in a round bottom flask using rotary evaporator under reduced pressure at $45^{\circ} \mathrm{C}$ for 4-6 h. The dry film was hydrated with $50 \mathrm{mM}$ HEPES buffer $\mathrm{pH} 6.5$ at $65^{\circ} \mathrm{C}$ for $45-60$ minutes. The multilamellar vesicles (MLVs) thus 
obtained were sized by extrusion through stacked polycarbonate membrane filters of varying pore diameters under high pressure to obtain small unilamellar vesicles (SUVs). The SUVs were characterized for particle size distribution (PSD), zeta potential, and total and entrapped paclitaxel content. The liposomes were stored at $5 \pm 3^{\circ} \mathrm{C}$ until further use.

\subsubsection{Determination of $\mathrm{Tg}^{\prime}$}

The glass transition of maximally freeze concentrated amorphous phase $\left(\mathrm{Tg}^{\prime}\right)$ of mixture of liposomes and sucrose was determined by differential scanning calorimetry (DSC) using a Q2000 differential scanning calorimeter equipped with refrigerated cooling system (TA Instruments, New Castle, DE). About 10-20 $\mu \mathrm{L}$ of the liposome mixture prior to lyophilization was placed in T-Zero aluminum pans and hermetically sealed. An empty pan prepared in a similar manner was used as reference. The samples were equilibrated at $25^{\circ} \mathrm{C}$ and then cooled at $5^{\circ} \mathrm{C} / \mathrm{min}$ to $-70^{\circ} \mathrm{C}$. After equilibration, the samples were heated at the rate of $10^{\circ} \mathrm{C} / \mathrm{min}$ to $25^{\circ} \mathrm{C}$.

\subsubsection{Lyophilization of Pac-LCL}

The Pac-LCL was mixed with equal volume of sucrose solution at different lipidsugar ratios. The lyophilization cycle was designed based on the glass transition temperature of maximally freeze-concentrated amorphous phase $\left(\mathrm{Tg}^{\prime}\right)$, determined by DSC. Lyophilization was carried out using a lab scale Virtis Genesis 25ES lyophilizer (The Virtis Company, Gardiner, NY). The primary drying temperature was selected based on $\mathrm{Tg}^{\prime}$ and the mixture was lyophilized in $2 \mathrm{cc}$ glass amber vials. The moisture content of the dry cake was monitored to ensure end point of secondary drying. The vesicle size, zeta potential, and total and entrapped paclitaxel content were monitored in samples reconstituted with HPLC grade water. The vials were stored at $5 \pm 3^{\circ} \mathrm{C}$ until further use. The gel to liquid crystalline transition temperature $\left(\mathrm{T}_{\mathrm{m}}\right)$ of the lipid bilayer mixture in the lyophilized formulations was determined by DSC. About $10 \mathrm{mg}$ of the lyophilized cake was placed in the aluminum pans. The samples were equilibrated at $25^{\circ} \mathrm{C}$ and then heated at the rate of $10^{\circ} \mathrm{C} / \mathrm{min}$ to $75^{\circ} \mathrm{C}$, and thermograms were recorded. To evaluate the effect of internal-external sucrose ratios, the liposomes were prepared with different amounts of sucrose added at the hydration step. The resulting liposomes were mixed with sucrose solutions containing different amounts of external sucrose and then lyophilized.

\subsubsection{Characterization of Liposomal Formulations}

The PSD (Z-avg) of the liposomes was determined using dynamic light scattering using Zetasizer Nano ZS (Malvern Instruments, Westborough, MA) equipped with 4.5 $\mathrm{mW}$ diode laser as a source of light operating at $670 \mathrm{~nm}$. Particle scattered photons were detected at an angle of $173^{\circ}$. The samples were suitably diluted with HPLC grade water for the determination of Z-avg. Three independent measurements were performed for 
each sample. Zeta potential was determined from electrophoretic mobility using samples suitably diluted with HPLC grade water. The lyophilized formulations were reconstituted with HPLC grade water prior to measurement of the PSD and zeta potential.

The loading efficiency of paclitaxel in the liposomes was determined by minicolumn centrifugation method using Sephadex G-25 macro spin columns (Harvard apparatus, Holliston, MA) similar to the method described by Zhang et al [249] with modifications. Briefly, the Sephadex G-25 particles in the column were suspended in $0.1 \% \mathrm{w} / \mathrm{v}$ TFA and were allowed to swell for 15 minutes. The columns were centrifuged at $1500 \mathrm{~g}$ at $25^{\circ} \mathrm{C}$ for 4 min using IEC CL31R Multispeed Centrifuge (Thermo Scientific, Asheville, NC) to remove TFA. About $100 \mu \mathrm{L}$ of placebo LCL was added and centrifuged to saturate the column. The column saturation with placebo liposomes was repeated for a total of three times. The gel columns were placed in fresh collection tubes for use with drug-loaded liposomes. The liposome samples were diluted with the dispersion medium of liposomes. About $100 \mu \mathrm{L}$ of the diluted sample was added to the gel column, and centrifuged for 4 minutes. The lyophilized liposomes were reconstituted with HPLC grade water prior to dilution. The eluate in the collection tube, containing only entrapped paclitaxel was analyzed by RPHPLC. Paclitaxel in the liposomes before passing through Sephadex G-25 was quantified for the total drug content. The HPLC system comprised of Waters 600 Controller, Waters 717 Plus Autosampler, and Waters 2996 Photodiode Array Detector (Waters Corporation, Milford, MA) was used. The RPHPLC analysis was performed using a Nova-Pak $\mathrm{C}_{18}$ column $(3.9 \times 150 \mathrm{~mm}, 4 \mu \mathrm{m})$ (Waters Corporation, Milford, MA). An isocratic elution was performed using acetonitrile-water in a ratio 55: $45 \mathrm{v} / \mathrm{v}$ at a flow rate of $1 \mathrm{~mL} / \mathrm{min}$, and detection wavelength of $230 \mathrm{~nm}$. The liposomes were digested with absolute ethanol, and suitably diluted before injection. The entrapment efficiency was calculated using the formula, Entrapment efficiency $=($ Entrapped paclitaxel $\mathrm{x}$ 100)/Total paclitaxel).

The moisture content of the lyophilized cake was determined using an Orion AF8 Volumetric Karl Fischer Titrator (Orion Research Inc., Boston, MA). The titrator was calibrated on each day of analysis with a known quantity of water. About $75 \mathrm{mg}$ of the dried sample was weighed accurately and added to the titration vessel. The titration was started after allowing one minute for extraction.

\subsubsection{Preparation and Lyophilization of Paclitaxel-Loaded cRGD Conjugated Targeted Long-Circulating Liposomes (Pac-RGD-TLCL)}

For the preparation of Pac-RGD-TLCL, a part (0.5 moles \%) of DSPE-PEG 2000 was replaced with maleimide DSPE-PEG 2000 and the liposomes were prepared as described in section 6.1.2 to obtain SUVs with reactive maleimide functional groups. The maleimidePac-LCL thus obtained was conjugated with cRGD peptide by incubation at $25^{\circ} \mathrm{C}$ for 12 $\mathrm{h}$. The reaction was quenched by the addition of 2-mercaptoethanol to block free maleimide. The unconjugated peptide and 2-mercaptoethanol remaining in the reaction mixture were removed from the liposomes by equilibrium dialysis through 10,000 MWCO Slide-A-Lyzer dialysis cassettes (Thermo Fisher Scientific, Rockford, IL) 
against $50 \mathrm{mM}$ HEPES buffer $\mathrm{pH} 6.5$ at $5 \pm 3^{\circ} \mathrm{C}$ overnight. The purified liposomes were mixed with sucrose solution and then lyophilized as described in 6.1.4. The liposomes were stored at $5 \pm 3^{\circ} \mathrm{C}$ until further use.

\subsubsection{Preparation and Lyophilization of Paclitaxel-Loaded Transferrin Conjugated Targeted Long-Circulating Liposomes (Pac-Tf-TLCL)}

For the preparation of Pac-Tf-TLCL, a part (0.01 moles \%) of DSPE-PEG 2000 was replaced with biotinylated DSPE-PEG ${ }_{2000}$ and the liposomes were prepared similar to 6.1.2 using phosphate buffered saline $\mathrm{pH} 7.2$ (PBS) instead of HEPES buffer. Transferrin was non-covalently conjugated to biotin on the distal end of the PEG spacers of the biotinylated SUVs using the procedure established in our laboratory [159]. Briefly, streptavidin was dissolved in PBS to prepare a $2 \mathrm{nM}$ solution. To the streptavidin solution in a scintillation vial, biotinylated liposomes was added, and mixed. The reaction mixture was incubated at $25^{\circ} \mathrm{C}$ for $30 \mathrm{~min}$, and the free streptavidin was removed by ultrafiltration using Centricon 100 centrifugal filter device (100 KD MWCO, Millipore, Bedford, MA) at $5000 \mathrm{~g}$ for $30 \mathrm{~min}$ to obtain purified streptavidin conjugated long-circulating liposomes (SALCL). Biotin labeled human transferrin was dissolved in PBS $(2 \mathrm{nM})$ and added to the SALCL, incubated at $25^{\circ} \mathrm{C}$ for $30 \mathrm{~min}$, and the free transferrin was removed by ultrafiltration. The purified liposomes were mixed with sucrose solution and then lyophilized as described in 6.1.4. The liposomes were stored at $5 \pm 3^{\circ} \mathrm{C}$ until further use.

\subsubsection{Stability Evaluation of Lyophilized Formulations of Pac-LCL, Pac-RGD- TLCL, and Pac-Tf-TLCL}

Stability of lyophilized formulations of Pac-LCL, Pac-RGD-TLCL, and Pac-TfTLCL was evaluated for three months. The formulations in $2 \mathrm{cc}$ amber glass vials were stored at $5 \pm 3^{\circ} \mathrm{C}$ for three months, and $25^{\circ} \mathrm{C}$ for one month. Samples were withdrawn at specific intervals and analyzed for total and entrapped paclitaxel, entrapment efficiency, PSD, zeta potential, and moisture content.

\subsection{RESULTS AND DISCUSSION}

\subsubsection{Effect of Sucrose on Paclitaxel Retention and PSD}

The stability of liposomes in dispersion form during storage is a major challenge in the development of liposomal formulations. Physical instability results in leakage of encapsulated drug, and fusion and/or aggregation with an increase in vesicle size. The hydrolysis or oxidation of the phospholipids during storage can be prevented by removing water from the liposomes by lyophilization. But, the interaction of water with phospholipids plays a key role in the formation of liposomes and its removal creates more challenges. Some carbohydrates interact by hydrogen bonding between their $-\mathrm{OH}$ group 
and polar head groups of phospholipids, and protect the liposomes during lyophilization [208]. It was shown that hydrogen bonding alters the spacing of the polar head groups and thereby decreases van der Waals interactions in the hydrocarbon chains. The net effect is improved physical stability of the liposomes. The protection effect was also attributed to their ability to replace water molecules normally hydrogen-bonded to the polar head groups of lipids [33]. Developments in analytical techniques such as high resolution ${ }^{1} \mathrm{H}-\mathrm{NMR}$ (nuclear magnetic resonance), Electron spin resonance (ESR) spin labeling and DSC studies [220, 221] showed that the mechanism of lyoprotection depends on the ability of these saccharides to replace water molecules of hydration by forming hydrogen bond with the polar phospholipid group at the same binding sites as water. In addition, water crystallizes during the freezing process to form ice crystals. At this stage, the vesicles and remaining solute are in a highly concentrated state which can induce fusion and aggregation. Inclusion of lyoprotectant can form an amorphous matrix around the vesicles and protect the vesicles from fusion and prevent rupture of the vesicles due to crystallization of ice. Koster et al showed that vitrification of sugars occurs in the intermembrane region, and the resulting glassy state mechanically hinders conformational changes of lipid bilayers [212, 227].

Trehalose is the most widely used lyoprotectant in the stabilization of liposomes [208, $217,274]$. In our preliminary evaluation of trehalose and sucrose as the lyoprotectants for liposomes (data not shown), we observed a better control on vesicle size with sucrose compared to trehalose. Our observations are in agreement with the results obtained by other investigators in the development of paclitaxel-loaded liposomes [249, 273]. In addition, Sundaramurthi and Suryanarayanan have demonstrated that trehalose has the tendency to undergo crystallization during lyophilization [275]. They suggested that partial crystallization occurs with annealing, and can be induced by other crystallizing solutes in the formulation such as buffer components. Even if nucleation is induced during lyophilization, crystal growth can occur during storage in the presence of residual moisture. Since crystallization during lyophilization can affect the integrity of the liposomes, we evaluated sucrose, a non-crystallizing solute as a lyoprotectant in the development of lyophilized formulation of Pac-LCL.

The lyophilization cycle was designed based on the Tg' determined by DSC. The heating profiles of mixtures of liposomes containing different concentrations of sucrose are shown in Figure 6-1. The $\mathrm{Tg}^{\prime}$ of these mixtures containing $50-450 \mathrm{mM}$ of sucrose was between -34 to $-39^{\circ} \mathrm{C}$. The $\mathrm{Tg}^{\prime}$ values decreased with an increase in sucrose concentrations up to $100 \mathrm{mM}$. Further increase in sucrose caused a reverse effect with an increase in the transition temperature. For lyophilization cycle, the liposome-sucrose mixtures were frozen to $-45^{\circ} \mathrm{C}$ and held at that temperature for 5 hours. The primary drying was started at $-40^{\circ} \mathrm{C}$. The product temperature, shelf temperature, and condenser temperature were monitored to determine the end point of primary drying. Then the temperature was slowly ramped up $10^{\circ} \mathrm{C}$ at a time to $20^{\circ} \mathrm{C}$ for the secondary drying. The end of secondary drying was monitored my measuring the moisture content of the lyophilized cake. The physical appearance of the cake, and ease of reconstitution with water were monitored as in-process quality control parameters. 


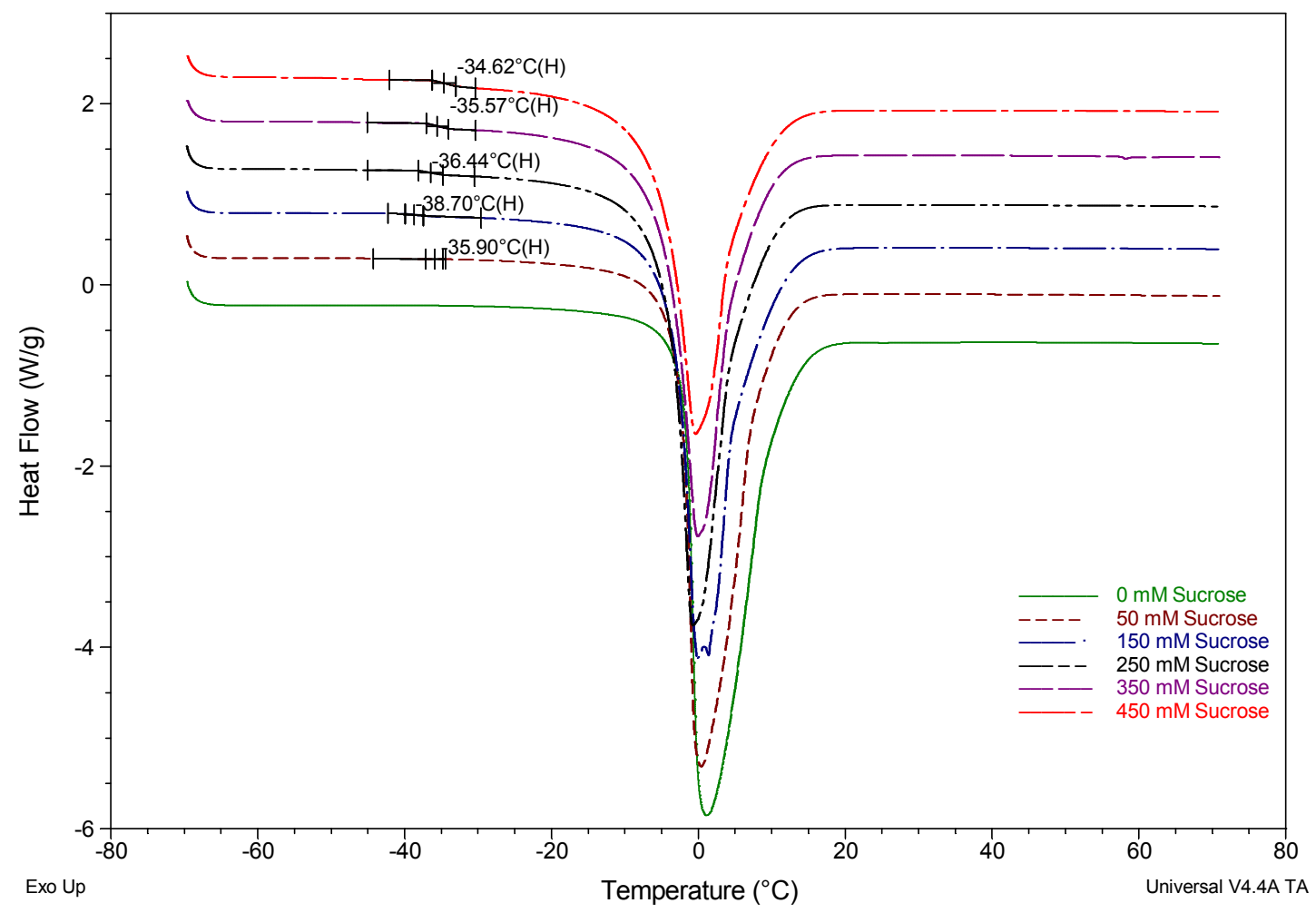

Figure 6-1. Effect of sucrose concentration on $\mathrm{Tg}^{\prime}$

Notes. The liposomes were mixed with sucrose solution at different lipid-sugar molar ratios. The samples were hermetically sealed in aluminum pans, and cooled at the rate of $5^{\circ} \mathrm{C} / \mathrm{min}$ to $-70^{\circ} \mathrm{C}$. After equilibration at $-70^{\circ} \mathrm{C}$, the samples were heated at the rate of $10^{\circ} \mathrm{C} / \mathrm{min}$ to $25^{\circ} \mathrm{C}$. The heating profile is shown in the figure. Heat flow is shown on an arbitrary scale. 
The pre-lyophilized liposomes were reconstituted for the determination of paclitaxel retention and PSD. Figure 6-2 shows the effect of sucrose concentration on paclitaxel retention in the Pac-LCL. Before lyophilization, the amount of free drug was low and the percent paclitaxel retained in the liposomes was approximately $90 \%$. When the liposomes were lyophilized without sucrose, leakage of paclitaxel was observed with an increase in the amount of free drug in the reconstituted dispersion by more than five-fold, and a sharp decline in percent paclitaxel retained in the liposomes. The formation of ice crystals during lyophilization might have caused rupture to the bilayer and resulted in loss of integrity of these liposomes. Secondly, the removal of water molecules which are necessary for maintaining the structure of the liposomes without replacing them with lyoprotectants might have altered the arrangement of phospholipids in the bilayer resulting in loss of integrity. The loss of integrity was restored with increasing concentrations of sucrose as lyoprotectant in the formulation. The maximum effect of lyoprotection was observed at $250 \mathrm{mM}$ of sucrose corresponding to a lipid-sugar ratio of 1:5. The free drug content in the reconstituted liposomal dispersion was almost equal to that observed before lyophilization suggesting the leakage of paclitaxel was completely prevented at that concentration. Further changes in lipid-sugar ratio did not show any appreciable effect on paclitaxel retention in the liposomes. These results suggest that sucrose at the lipid-sugar ratios equal to or below 1:5 efficiently replaced water molecules hydrogen bonded to the phospholipids of the bilayer and helped to maintain the integrity of paclitaxel-loaded liposomes during lyophilization.

The gel to liquid crystalline transition temperature also plays a key role in maintaining the integrity of the liposomes during dehydration and rehydration [217]. The phospholipids in the rehydrated form remain in liquid crystalline state. If the phospholipids in the dehydrated state remain in gel phase at room temperature, the conversion from gel to liquid crystalline state occurs. The liposomes are prone to leak encapsulated contents during this transition. Lyoprotectants reduce the $\mathrm{T}_{\mathrm{m}}$ of the phospholipids in the bilayer on lyophilization. If such reduction brings the $T_{m}$ below room temperature, the dehydrated liposomes stay at liquid crystalline temperature and rehydration will not result in the transition, and thus leakage of the encapsulated drug can be avoided. The effect of sucrose on $T_{m}$ of the phospholipids of the bilayer of Pac-LCL is shown in Figure 6-3. The DSC thermograms obtained from the lyophilized samples shows that there is a decrease in the $T_{m}$ of the phospholipids with an increase in the concentration of sucrose. But, the $T_{m}$ of the formulations containing $250 \mathrm{mM}$ sucrose was above room temperature suggesting that the phospholipids in the bilayer of Pac-LCL at room temperature will remain in the gel state and a transition during rehydration is inevitable. It is interesting to note that paclitaxel is a lipophilic drug and for thermodynamic reasons will stay within the lipophilic bilayers unless forced/precipitated out of the liposomes. On the other hand, the transition temperature will play a key role on the retention of hydrophilic drugs in the liposomes because these drugs can easily leak out of the liposomes if the integrity of the bilayer is compromised during the transition. Nevertheless, the change in $\mathrm{T}_{\mathrm{m}}$ indicates that there is a direct interaction between the phospholipid head groups and sucrose which is responsible for lyoprotection. 


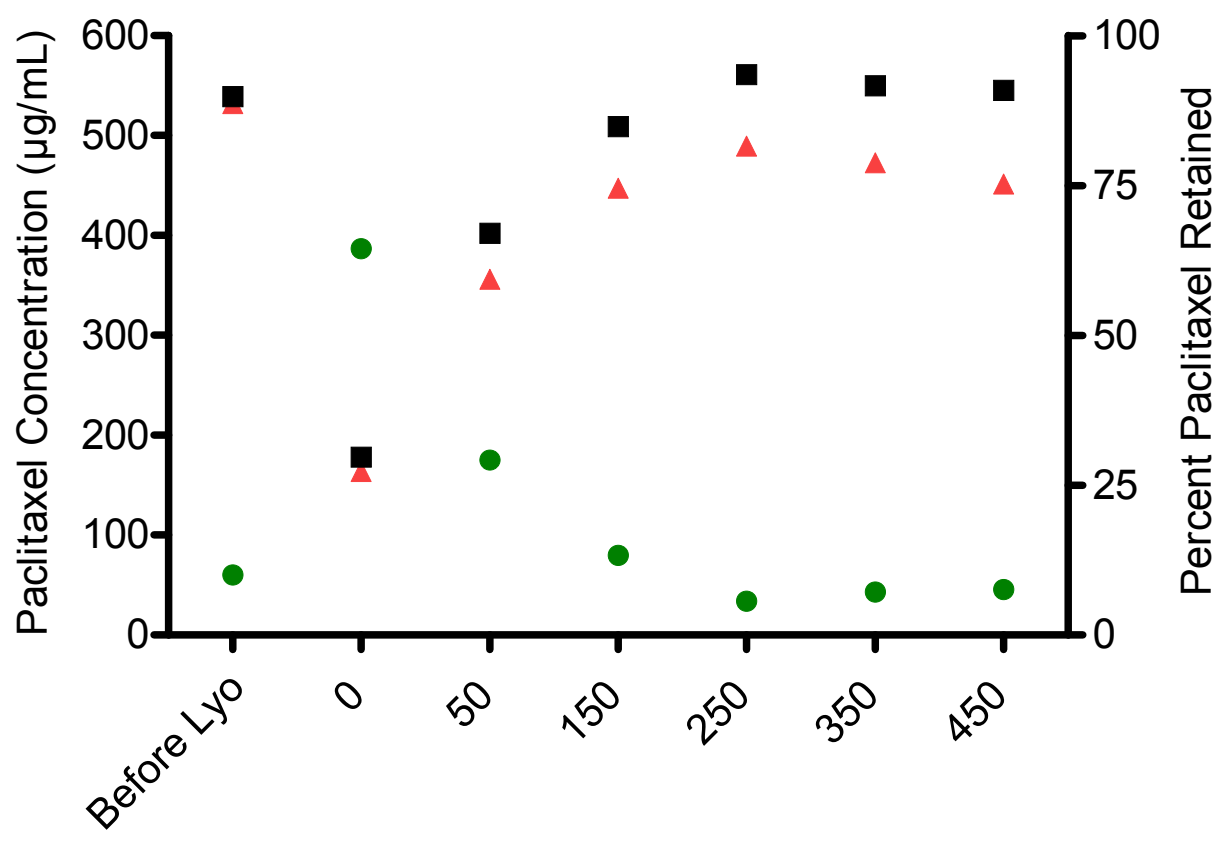

Sucrose Concentration (mM)

Figure 6-2. Effect of sucrose concentration on the effect of paclitaxel retention in the liposomes during lyophilization

Notes. The liposomes were mixed with sucrose solution at different lipid-sugar molar ratios and then lyophilized. The paclitaxel retained in the liposomes was determined before and after lyophilization. The lyophilized formulations were reconstituted with HPLC grade water for the determination of paclitaxel retention. For the determination of paclitaxel retention, the liposomes were subjected to minicolumn centrifugation in Sephadex G-25 macro spin columns to separate free paclitaxel from total paclitaxel. The eluate containing entrapped paclitaxel was digested with ethanol, and quantified by RPHPLC method. Free paclitaxel was calculated by subtracting entrapped paclitaxel from the total paclitaxel content quantified from samples before gel filtration. The results indicate the mean \pm standard deviation of three injections ( $\Delta$ Paclitaxel retained in the

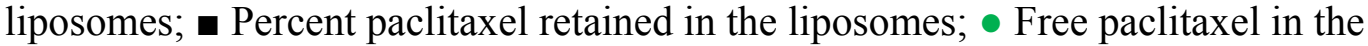
dispersion). 


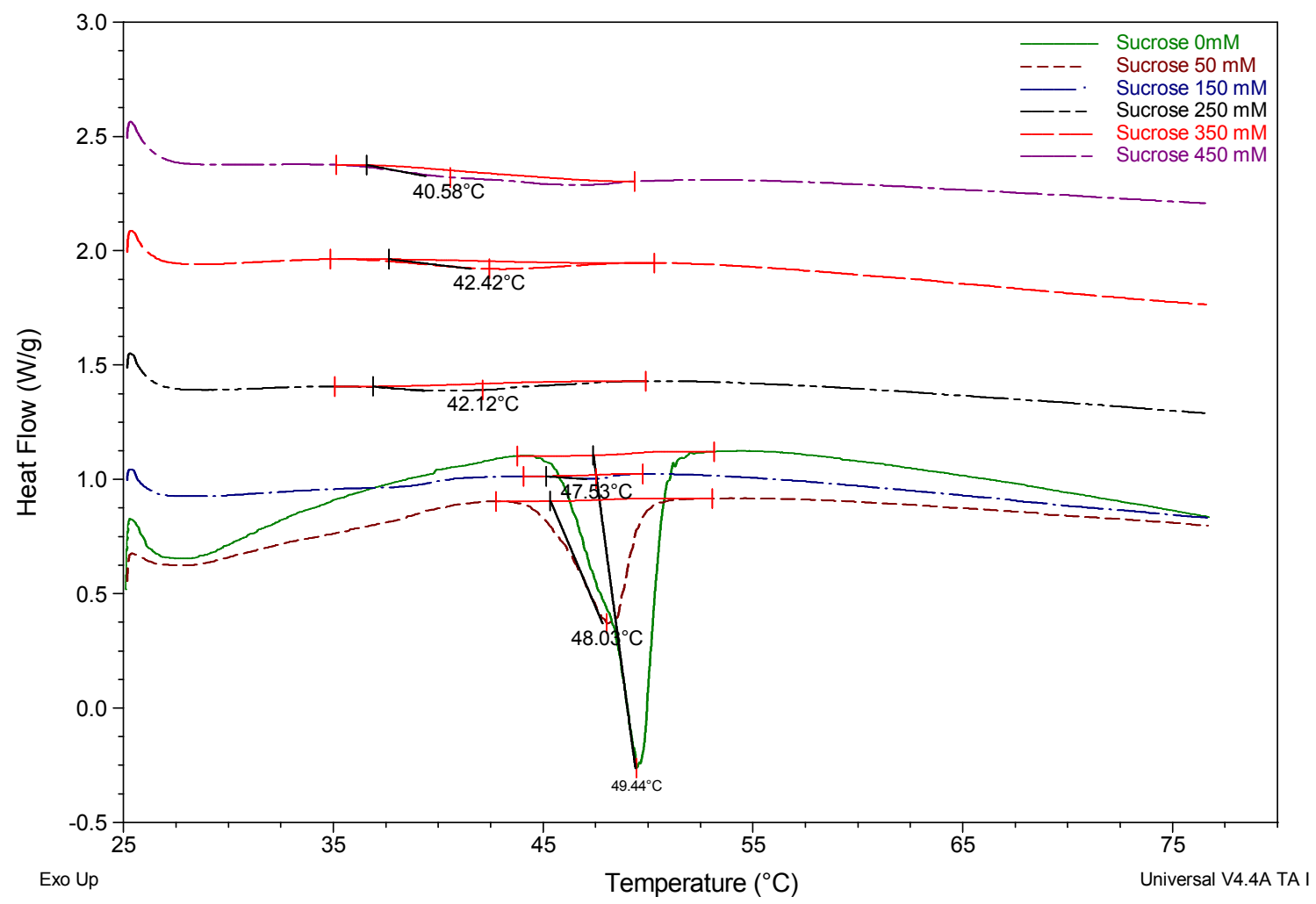

Figure 6-3. Effect of sucrose concentration on $\mathrm{T}_{\mathrm{m}}$ of the lipids in the bilayer

Notes. The liposomes were mixed with sucrose solution at different lipid-sugar molar ratios and then lyophilized. About $10 \mathrm{mg}$ of the lyophilized cake was placed in T-zero aluminum pans. The samples were equilibrated at $25^{\circ} \mathrm{C}$ and then heated at the rate of $10^{\circ} \mathrm{C} / \mathrm{min}$ to $75^{\circ} \mathrm{C}$. The resulting thermograms are shown in the figure. Heat flow is shown on an arbitrary scale. 
The effect of lyoprotectant sucrose on the PSD of reconstituted liposomal dispersions compared to their PSD before lyophilization is presented in Figure 6-4. The particle size increased to almost seven-fold for the liposomes lyophilized without lyoprotectant sucrose suggesting that fusion and aggregation of liposomes occurred during lyophilization. The PSD results showed higher variability and a polydispersity index (PDI) of $0.726 \pm 0.275$. The addition of sucrose in the formulation for lyophilization minimized this increase in size with increasing concentrations of sucrose. Similar to the observations with paclitaxel retention in the reconstituted liposomal dispersion, the maximum effect of lyoprotectant was observed at sucrose concentration of $250 \mathrm{mM}$ corresponding to the lipid-sugar ratio of 1:5. The vesicle size after lyophilization was slightly higher than the liposomes prior to lyophilization. The particle size distribution was unimodal with a PDI of $0.250 \pm 0.011$. Further decrease in the lipid to sugar ratio did not produce any appreciable effect on the PSD. Similar to the effect on integrity of the liposomes to prevent leakage, sucrose worked as an efficient lyoprotectant to minimize fusion and aggregation of liposomes and thereby minimized the increase in size after lyophilization.

The effect of lyoprotectant is maximum when it is present on both internal and external portions of the bilayer [33]. The sucrose concentration of $350 \mathrm{mM}$ corresponding to a lipid-sugar ratio of 1:7 was selected for further evaluation of internal to external ratio of sucrose. When the total $350 \mathrm{mM}$ of sucrose is divided in to 1:6 or 2:5 in the internalexternal part of the bilayer, there would be enough sucrose (at least $250 \mathrm{mM}$ ) available in the external portion of the bilayer of liposomes to provide maximum lyoprotectant effect. The effect of internal-external ratio of sucrose on the PSD of the reconstituted liposomal dispersions compared to their PSD before lyophilization is shown in Figure 6-5. Inclusion of sucrose in the internal portion of the bilayer provided additional protection to the Pac-LCL during lyophilization as evidenced by the decrease in size of the reconstituted liposomal dispersions with an increase in internal sucrose. The internal to external-external sucrose ratio was not decreased further because the minimum required amount of external sucrose of $250 \mathrm{mM}$ cannot be obtained with a total sucrose concentration of $350 \mathrm{mM}$. In addition, literature reports support the use of only a small fraction of lyoprotectant in the internal portion of the bilayer since the use of higher amounts of internal lyoprotectant has no effect on apparent stability of the liposomes $[226,232]$. The internal-external sucrose ratio of 2:5 was selected as optimum with a total sucrose concentration of $350 \mathrm{mM}$ corresponding to a lipid-sugar ratio of 1:7 was selected as optimum for further development.

\subsubsection{Development of Lyophilized Targeted Liposomes}

The optimized composition for lyophilization was used for the preparation of lyophilized targeted liposomes. The optimum sucrose concentration of $350 \mathrm{mM}$ with an internal-external ratio of 2:5 provided adequate protection for Pac-RGD-TLCL, and PacTf-TLCL during lyophilization. The presence of either cRGD or transferrin on the surface of the liposomes did not affect the drug retention or size during lyophilization. Similar to Pac-LCL, a slight but insignificant increase in vesicle size was observed with 


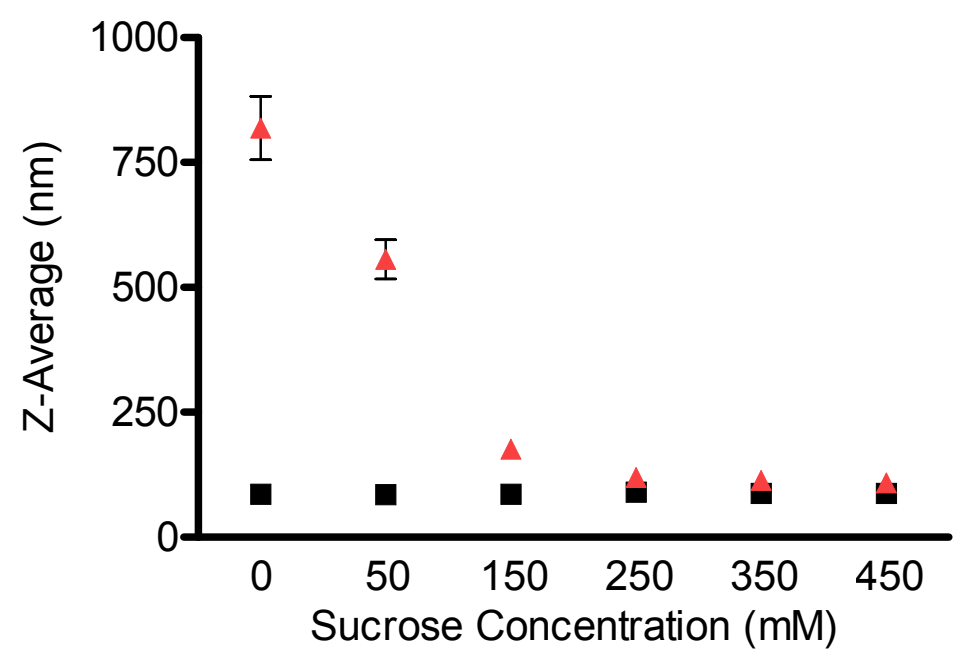

Figure 6-4. Effect of sucrose concentration on PSD of paclitaxel-loaded liposomes during lyophilization

Notes. The liposomes were mixed with sucrose solution at different lipid-sugar molar ratios and then lyophilized. The PSD of the liposomes was determined using dynamic light scattering. The lyophilized formulations were reconstituted with HPLC grade water for the determination of PSD. The results indicate mean \pm standard deviation of three independent measurements ( $\boldsymbol{\sim}$ PSD before lyophilization; $\triangle$ PSD after lyophilization determined from reconstituted samples). 


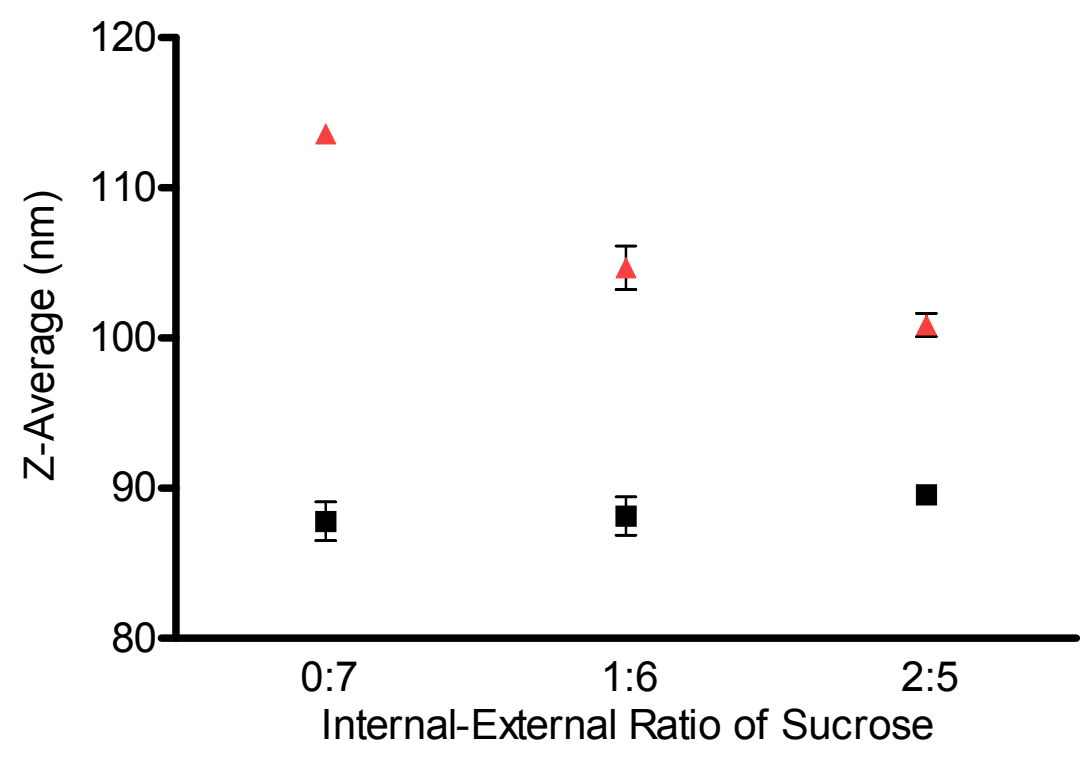

Figure 6-5. Effect of internal-external ratio of sucrose on PSD during lyophilization

Notes. The liposomes were prepared with different internal sucrose concentrations added at the hydration step and then mixed with sucrose solution to produce different internal-external sucrose ratios. The PSD of the liposomes was determined using dynamic light scattering. The lyophilized formulations were reconstituted with HPLC grade water for the determination of PSD. The results indicate mean \pm standard deviation of three independent measurements ( PSD before lyophilization; $\boldsymbol{\Delta}$ PSD after lyophilization determined from reconstituted samples). 
the reconstituted samples of targeted liposomes compared to their size before lyophilization. The paclitaxel retention was unaffected at the sucrose ratio used in the preparation of lyophilized Pac-RGD-TLCL and Pac-Tf-TLCL. The moisture content of the lyophilized cake was less than $1 \% \mathrm{w} / \mathrm{v}$.

\subsubsection{Stability of Lyophilized Pac-LCL, Pac-RGD-TLCL, and Pac-Tf-TLCL}

The stability of the lyophilized formulations of Pac-LCL, Pac-RGD-TLCL, and PacTf-TLCL were evaluated in $2 \mathrm{cc}$ amber vials at $5 \pm 3^{\circ} \mathrm{C}$ for three months and $25^{\circ} \mathrm{C}$ for 1 month. The stability data for lyophilized Pac-LCL is presented in Figure 6-6. The moisture content of the lyophilized cake was maintained below $1 \% \mathrm{w} / \mathrm{v}$ through the testing period. The paclitaxel retained in the liposomes and percent retained were within acceptable limit of $10 \%$ of initial at both the temperature conditions. The particle size and zeta potential were not affected at $5 \pm 3^{\circ} \mathrm{C}$, but an appreciable increase in size was observed at $25^{\circ} \mathrm{C}$ suggesting that these liposomes undergo fusion or aggregation at room temperature. Nevertheless, the Pac-LCL maintained its size, charge, and integrity through the evaluation period when stored at refrigerated conditions. The stability data for PacRGD-TLCL and Pac-Tf-TLCL is presented in Figure 6-7 and Figure 6-8 respectively. These targeted liposomes also exhibited a similar behavior in the stability as Pac-LCL. These targeted liposomes maintained integrity at $5 \pm 3^{\circ} \mathrm{C}$ as well as $25^{\circ} \mathrm{C}$. An increase in size of approximately $20-40 \mathrm{~nm}$ was observed with both the targeted liposomes within one month of storage at $25^{\circ} \mathrm{C}$ but there was no significant change in size or charge at refrigerated conditions. The stability data suggests that Pac-LCL, Pac-RGD-TLCL, and Pac-Tf-TLCL lyophilized formulation must be stored at $5 \pm 3{ }^{\circ} \mathrm{C}$ for a better shelf-life without any compromise on particle size distribution

\subsection{SUMMARY AND CONCLUSIONS}

The liposomal formulations containing paclitaxel were lyophilized to improve longterm stability during storage. The Pac-LCL lyophilized without any lyoprotectant showed a loss of integrity resulting in high concentration of free paclitaxel in the dispersion. Sucrose was used as a lyoprotectant to stabilize the liposomes during lyophilization. The optimum concentration of sucrose to minimize paclitaxel leakage was found to be 250 $\mathrm{mM}$ corresponding to a lipid-sucrose ratio of 1:5. Further increase in the concentration of sucrose in the formulation did not produce any appreciable effect on paclitaxel retention. A similar effect as in paclitaxel retention was observed with particle size distribution of liposomes lyophilized in presence of different concentrations of sucrose. When the liposomes were lyophilized without sucrose the particle size increased to above $700 \mathrm{~nm}$. Increasing concentrations of sucrose minimized this increase in size. A sucrose concentration of $250 \mathrm{mM}$ provided adequate protection to the liposomes during lyophilization and minimized fusion and aggregation. To evaluate the internal-external ratio of sucrose, $350 \mathrm{mM}$ of sucrose was used and internal-external ratios of 0:1, 1:6, and 2:5 were studied. The additional protection to the inner portion of the bilayer further minimized the particle size increase after lyophilization. Since only a small fraction of 

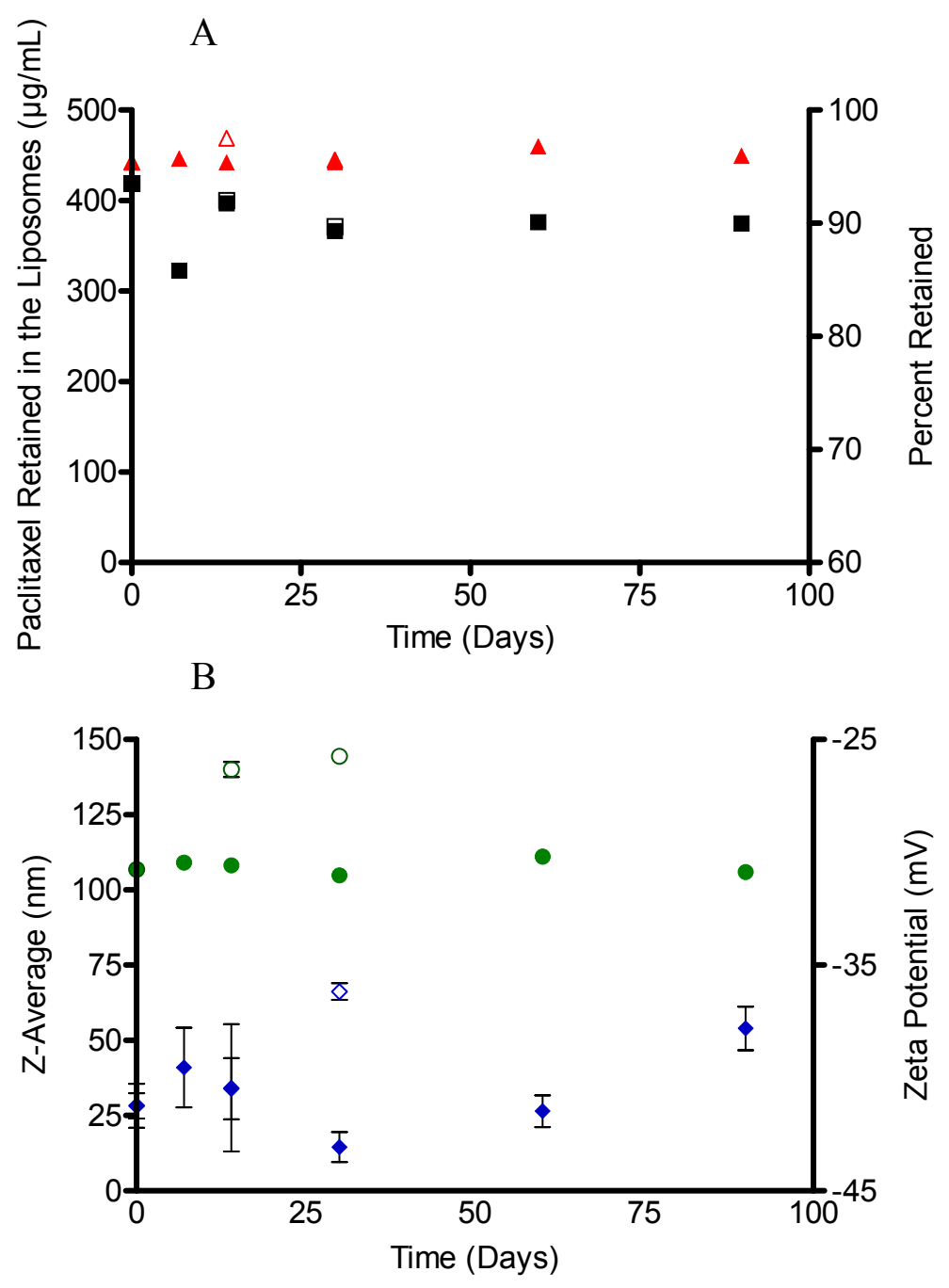

Figure 6-6. Stability of lyophilized Pac-LCL formulation

Notes. The Pac-LCL was stored at $5 \pm 3^{\circ} \mathrm{C}$ (solid symbols) and $25^{\circ} \mathrm{C}$ (open symbols) in 2 cc amber vials. Samples were withdrawn at specific intervals, reconstituted with HPLC water, and analyzed for paclitaxel retention, PSD, and zeta potential. A. For the determination of paclitaxel retention, the samples were subjected to minicolumn centrifugation in Sephadex G-25 macro spin columns to separate free paclitaxel from total paclitaxel. The eluate containing entrapped paclitaxel was digested with ethanol, and quantified by RPHPLC method. The results indicate the mean \pm standard deviation for three injections ( $\Delta$ Paclitaxel retained in the liposomes; - Percent paclitaxel retained in the liposomes). B. For PSD, and zeta potential, the results indicate mean \pm standard deviation of three independent measurements (• PSD; $\diamond$ Zeta potential). 

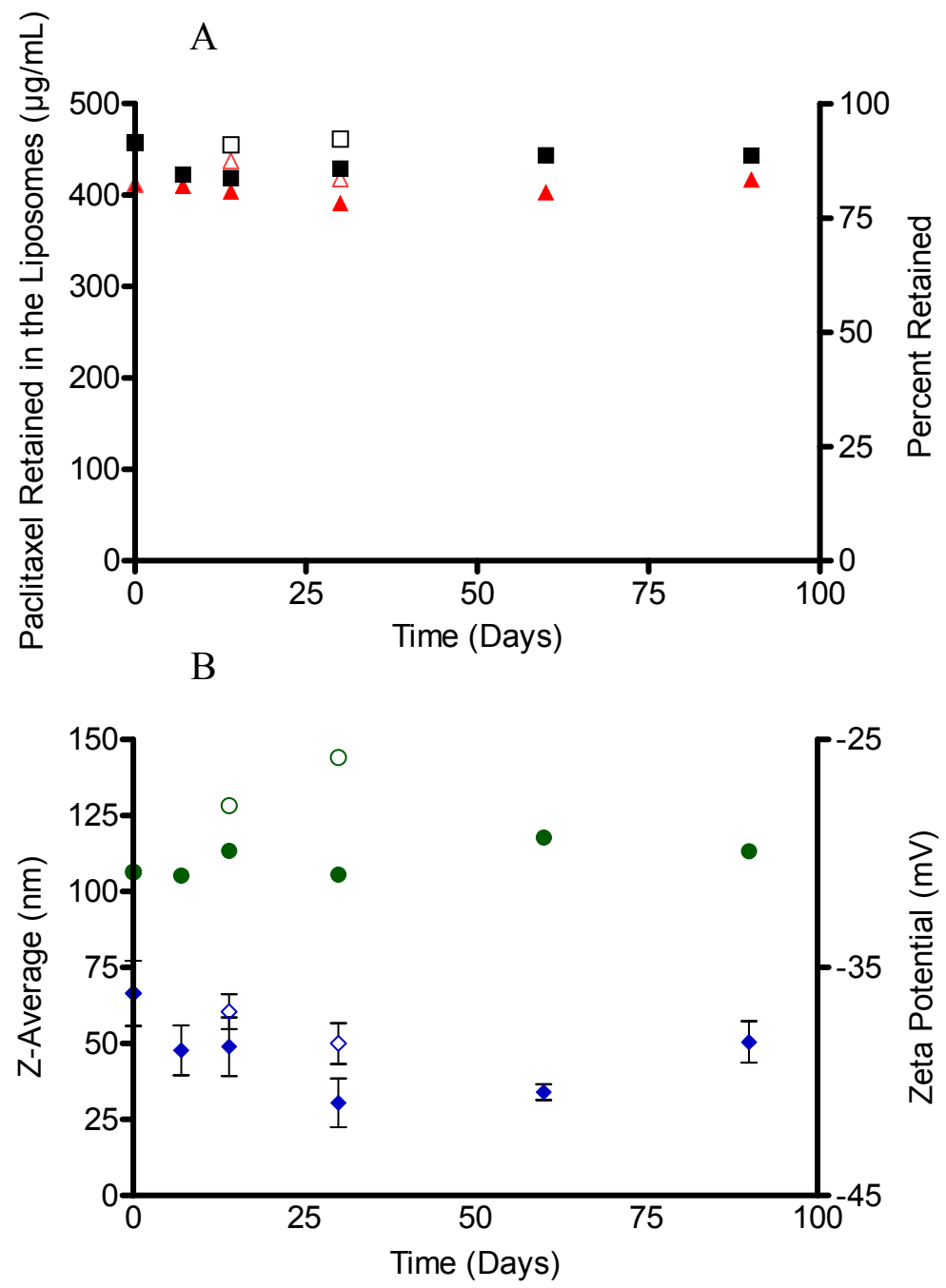

Figure 6-7. Stability of lyophilized Pac-RGD-TLCL

Notes. The Pac-RGD-TLCL was stored at $5 \pm 3^{\circ} \mathrm{C}$ (solid symbols) and $25^{\circ} \mathrm{C}$ (open symbols) in 2 cc amber vials. Samples were withdrawn at specific intervals, reconstituted with HPLC water, and analyzed for paclitaxel retention, PSD, and zeta potential. A. For the determination of paclitaxel retention, the samples were subjected to minicolumn centrifugation in Sephadex G-25 macro spin columns to separate free paclitaxel from total paclitaxel. The eluate containing entrapped paclitaxel was digested with ethanol, and quantified by RPHPLC method. The results indicate the mean \pm standard deviation for three injections ( $\Delta$ Paclitaxel retained in the liposomes; - Percent paclitaxel retained in the liposomes). B. For PSD, and zeta potential, the results indicate mean \pm standard deviation of three independent measurements (• PSD; $\bullet$ Zeta potential). 

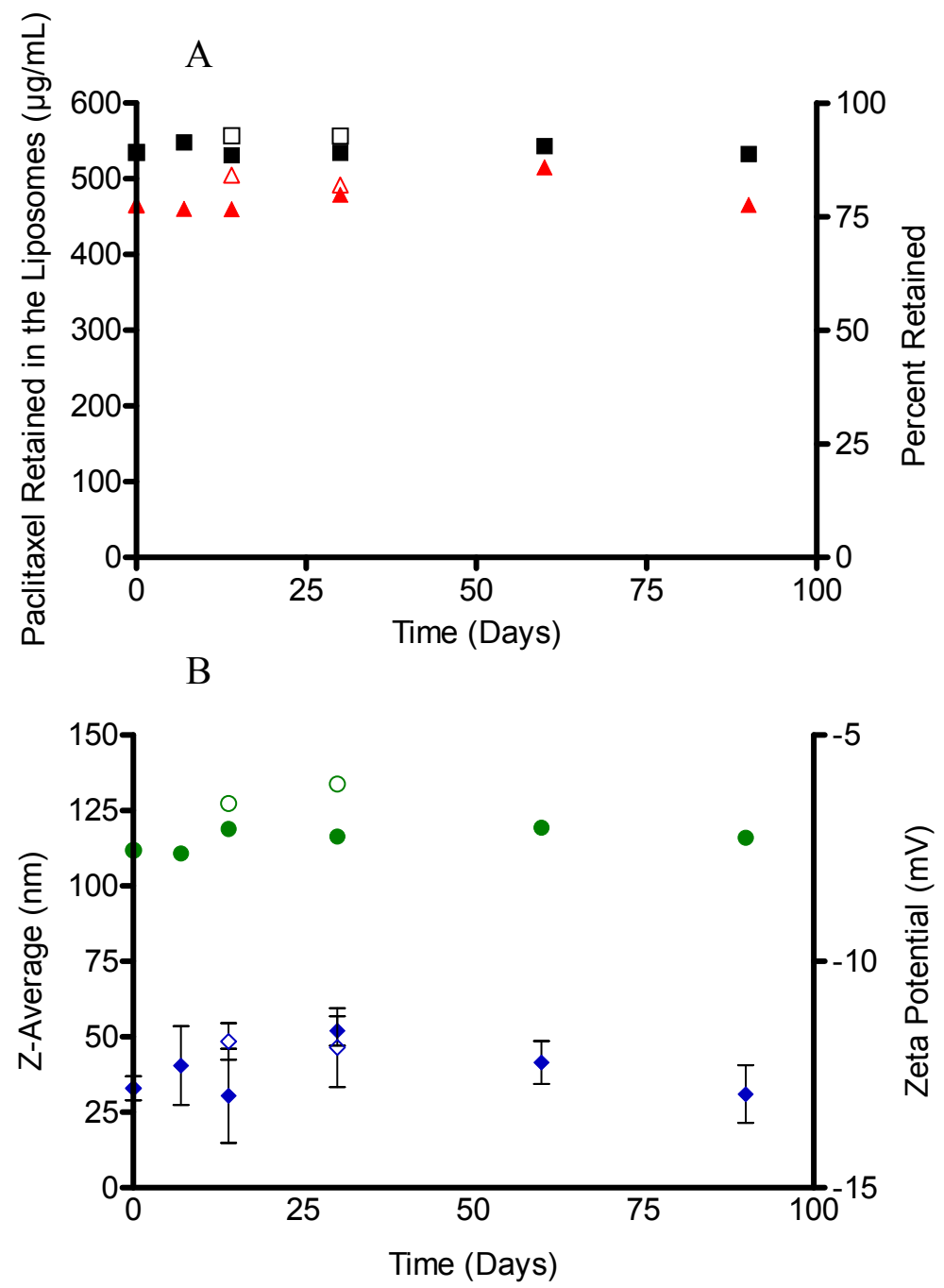

Figure 6-8. Stability of lyophilized Pac-Tf-TLCL

Notes. The Pac-Tf-TLCL stored at $5 \pm 3^{\circ} \mathrm{C}$ (solid symbols) and $25^{\circ} \mathrm{C}$ (open symbols) in $2 \mathrm{cc}$ amber vials. Samples were withdrawn at specific intervals, reconstituted with HPLC water, and analyzed for paclitaxel retention, PSD, and zeta potential. A. For the determination of paclitaxel retention, the samples were subjected to minicolumn centrifugation in Sephadex G-25 macro spin columns to separate free paclitaxel from total paclitaxel. The eluate containing entrapped paclitaxel was digested with ethanol, and quantified by RPHPLC method. The results indicate the mean \pm standard deviation for three injections ( $\Delta$ Paclitaxel retained in the liposomes; Percent paclitaxel retained in the liposomes). B. For PSD, and zeta potential, the results indicate mean \pm standard deviation of three independent measurements (• PSD; $\bullet$ Zeta potential). 
internal sucrose is necessary for the lyoprotection the internal-external ratio of 2:5 was selected as optimum. The composition for lyophilization was optimized for Pac-LCL and the optimum composition was used in the lyophilization of Pac-RGD-TLCL, and Pac-TfTLCL. The presence of targeting ligands on the surface of these targeted liposomes did not affect the integrity, size, and charge and were similar to Pac-LCL. The stability data showed that these liposomes were stable in refrigerated condition for three months. The particle size increased by $20-40 \mathrm{~nm}$ in the samples stored at $25^{\circ} \mathrm{C}$ suggesting that these liposomes undergo fusion and aggregation at room temperature. The freeze dried cake maintained its appearance and the moisture content was within acceptable limits through the testing period. The paclitaxel retention, particle size, and zeta potential were maintained within acceptable limits for three months in refrigerated conditions. A stable lyophilized formulation was developed for paclitaxel-loaded long-circulating liposomes and targeted long-circulating liposomes. 


\section{CHAPTER 7. SUMMARY AND CONCLUSIONS}

Breast cancer is a serious health care concern affecting nearly 200,000 women a year in the United States. Paclitaxel is one of the most effective drugs used in the treatment of breast cancer but it has drawbacks of serious adverse effects due to formulation components or due to exposure of normal tissues to free drug. Therefore, an effective drug delivery system that can deliver paclitaxel locally at the tumor sites following intravenous administration without affecting normal tissues was sought. Liposomes are attractive choice for this purpose because they are biocompatible in nature, and can accommodate drugs of hydrophilic or lipophilic nature. In addition, the flexibility in design allows surface modifications to improve in vivo circulation times, and for targeting. Passive targeting approach with liposomes can take advantage of the leaky nature of the tumors for selective accumulation. Combination of passive targeting approach with an active targeting approach with selective targeting ligands to improve the cellular uptake of the liposomes at the tumor sites can have a significant impact on overall effectiveness of the therapy. The objective of this dissertation work was to develop and evaluate paclitaxel-loaded targeted long-circulating liposomal formulations for drug delivery to breast cancer. In addition to targeted therapy, attempts were made to develop ultrasound active liposomes that can be visualized by ultrasound and disintegrated with high intensity ultrasound pulses. Ultrasound destruction mediated release, and improved vascular permeability is also expected to improve the effectiveness of the therapy.

A prototype formulation of cRGD conjugated echogenic liposomes was developed to target $\alpha_{\mathrm{v}} \beta_{3}$ integrin receptors over expressed in actively proliferating cells of the tumor vasculature. Perfluoropropane, a lipophilic gas commonly used in ultrasound contrast agents was filled into the bilayer of liposomes by freeze drying gas entrapment method in presence of mannitol. Mannitol undergoes crystallization during the warming phase of lyophilization and induces rupture of the bilayer through which gas penetrates the bilayer of liposomes. This process results in an increase in size of the liposomes to $\sim 800 \mathrm{~nm}$ which makes it unsuitable for drug delivery applications. So, attempts were made to control the size of these liposomes to $150-200 \mathrm{~nm}$ by controlling the crystallization of mannitol. The use of non-crystallizing solute sucrose at a mannitol-sucrose ratio of 4:3 minimized the crystallization of mannitol and thereby minimized the increase in size of these echogenic liposomes during lyophilization. Although, surfactant poloxamer 188 did not show any effect on crystallization, its use at concentrations above $0.05 \% \mathrm{w} / \mathrm{v}$ minimized the increase in size after lyophilization due to monolayer coating and steric effects on the surface. The echogenic formulations containing mannitol alone showed more than two-fold higher echogenicity compared the formulations containing mannitol and sucrose. The attachment of cRGD to the surface of the liposomes through PEG spacer did not affect size, charge or echogenicity of the formulation. The optimized formulation with a size of $\sim 150 \mathrm{~nm}$, and a zeta potential of $\sim-30 \mathrm{mV}$ was used as a platform for the development of paclitaxel-loaded echogenic liposomes. 
Paclitaxel loading in the bilayer of liposomes and retention of the encapsulated drug in the bilayer is important to achieve long term stability of the liposomes. The liposomal formulation used in the preparation of prototype formulation was optimized to increase paclitaxel loading. Cholesterol content showed a significant negative influence on paclitaxel loading with highest loading achieved at 5 moles \%. Saturated lipid content also showed a negative effect on paclitaxel loading. A HSPC-EPC ratio of 1:5 was selected as an optimum ratio. Total lipid concentration showed proportionate increase in loading with an increasing lipid concentration. A total lipid concentration of $100 \mathrm{mM}$ was used in the optimized formulation. Drug-lipid ratio also showed a proportionate increase in loading with an increase in the amount of paclitaxel. But, paclitaxel precipitated out of formulations with higher amounts of paclitaxel. Short-term stability studies with different ratios showed that the drug-lipid ratio of 1:60 was optimum for adequate physical stability of these long-circulating liposomes. The optimized formulation with paclitaxel loading of $\sim 1.2 \mathrm{mg} / \mathrm{mL}$ and a size of $100 \pm 20 \mathrm{~nm}$ maintained its integrity, size and charge at $5 \pm 3^{\circ} \mathrm{C}$ for three months.

The optimized paclitaxel-loaded long-circulating liposomes was used for the preparation of echogenic liposomes. When these liposomes were lyophilized in presence of mannitol-sucrose ratio of $4: 3$, and a poloxamer concentration of $0.15 \% \mathrm{w} / \mathrm{v}$, optimized composition from the prototype formulation, the size of the liposomes increased by more than four-fold suggesting further optimization is necessary. So attempts were made to minimize the increase in size with different lipid-sugar ratios with sucrose alone in presence of poloxamer. A decrease in lipid-sugar ratio minimized the increase in size up to the ratio 1:7, and further decrease did not show any influence. The inclusion of sucrose in the internal portion of the bilayer at an internal-external ratio of 1:6 helped minimizing the increase in size due to lyoprotection of the internal portion of the bilayer. Number of cycles used in the preparation of liposomes showed an influence on the PDI of the liposomes after lyophilization. The change in PDI after lyophilization was lower in liposomes prepared with one extrusion cycle. Mannitol was introduced in the formulation at the concentration of 50,100 , and $150 \mathrm{mM}$ to evaluate its influence on size and echogenicity of these liposomes. As expected, an increase in mannitol concentration caused an increase in size and PDI. The in vitro echogenicity evaluation showed that all the formulations containing mannitol showed $\sim 50$-fold increase in MGSV compared to background. The formulation that contained sucrose alone also showed $\sim 20$-fold increase in MGSV. Although mannitol containing formulations showed higher echogenicity, mannitol was excluded from the formulations to have better control on size and PDI. The cRGD peptide was conjugated to the surface of these liposomes through PEG spacers. These paclitaxel-loaded targeted echogenic liposomes of size 150-200 nm with a paclitaxel loading of $\sim 500 \mu \mathrm{g} / \mathrm{mL}$ was stable at $5 \pm 3^{\circ} \mathrm{C}$ for three months. The ability to disintegrate these echogenic liposomes with ultrasound pulses was evaluated by an in vitro method using mouse tail vein infusion catheter immersed in water filled in a plexiglass tank. The decrease in contrast mean power at the ROI showed that these echogenic liposomes can be disintegrated with high intensity ultrasound pulses. These targeted echogenic liposomes containing paclitaxel has the potential for improving the outcome of therapy of breast cancer and other solid tumors. Attempts were made to establish an in vivo animal model for the evaluation of these paclitaxel-loaded echogenic 
liposomes. Difficulties were experienced in visualizing these echogenic liposomes in the mouse xenograft model, which require further investigation. So, the gas-filled longcirculating liposomes were not used further for the in vivo experiments.

The biodistribution of cRGD conjugated liposomes was evaluated in mouse xenografts bearing MDA-MB-231 breast cancer by NIRF imaging with liposomes containing a fluorescent dye DiR. The results showed that the accumulation of DiRRGD-TLCL was lower than DiR-LCL, and slightly higher but not significantly different than DiR solution formulation. The total efficiency for the ROI measurements in the peritoneal cavity of the animals treated with DiR-RGD-TLCL was significantly higher compared to non targeted liposomes suggesting these targeted liposomes are cleared rapidly by the RES comprised of Kupffer cells of the liver and fixed macrophages of the spleen. The higher accumulation in the spleen was due to the expression of $\alpha_{v} \beta_{3}$ integrin receptors by the mononuclear cells of spleen. Quantification of paclitaxel in the tumor tissues after 24 hours of intravenous administration showed a comparable accumulation of Pac-RGD-TLCL and Pac-LCL for a size $~ 160 \mathrm{~nm}$. But, the accumulation was less than that of the solution formulation. A decrease in size to $\sim 80 \mathrm{~nm}$ showed a significantly higher blood levels and tumor accumulation emphasizing the importance of the size of these liposomes in tumor accumulation of paclitaxel. The antitumor efficacy of these tumor vasculature targeted liposomes was compared with tumor cell targeted liposomes using transferrin directed against TfR 1 receptors over expressed by the tumor cells. The results showed that all the paclitaxel containing formulations were effective in reducing the percent increase in tumor size compared to normal saline control at 39 days post tumor inoculation. The efficacy was comparable for Pac-RGD-TLCL, Pac-LCL, and paclitaxel solution formulation with tumor volumes of $\sim 60-70 \%$ of the control treatment. In contrast, the tumor cell targeted Pac-Tf-TLCL showed a significantly higher antitumor activity compared to all other treatments with the tumor volumes of $\sim 30 \%$ of the control treatment on 39 days post tumor inoculation. The tumor vasculature targeting did not produce an appreciable effect compared to non targeted liposomes possibly due to limited expression of $\alpha_{v} \beta_{3}$ integrin receptors in the vasculature of well established tumors, and due to higher clearance of these liposomes by binding of cRGD to these receptors expressed by mononuclear cells of the spleen. Nevertheless, tumor vasculature targeting has a potential for improving therapeutic efficacy in breast cancer with metastasis, and primary tumors with fast developing vasculature. In this animal model studied, tumor cell targeting produced a higher antitumor efficacy than tumor vasculature targeting because this strategy utilized the active process involved in Fe transport for targeting. Since TfR1 is over expressed by proliferating tumor cells, receptor mediated endocytosis of Pac-TfTLCL by these cells was enhanced, which in turn improved the efficacy of the treatment.

The physical instability of the liposomal dispersions during storage is a major concern in liposomal formulation development. Removal of water from the dispersion by lyophilization can improve the long term stability, and thereby instabilities caused by hydrolysis or oxidation can be prevented. But, removal of water which is responsible for maintaining the structure of the liposomes presents more challenges and requires careful optimization of the formulation and process. The liposomes lyophilized without lyoprotectant showed more than five-fold increase in free drug, and more than seven-fold 
increase in size. So, the Pac-LCL was lyophilized using a non-crystallizing solute sucrose as a lyoprotectant. The leakage of paclitaxel in the lyophilized samples was minimized with increasing concentrations of sucrose and the minimum concentration required was found to be $250 \mathrm{mM}$ corresponding to a lipid-sugar ratio of 1:5. Similarly, the increase in size during lyophilization was minimized by sucrose at lipid-sugar ratios of 1:5 or lower. Inclusion of sucrose at an intravesicular-extravesicular ratio of 2:5 provided better protection to the internal portion of the bilayer and minimized the increase in particle size. The reconstituted pre-lyophilized Pac-LCL was $100 \pm 20 \mathrm{~nm}$ in diameter with $90 \%$ paclitaxel retention. The optimized composition was used for the preparation of lyophilized Pac-RGD-TLCL, and Pac-Tf-TLCL. The lyophilized targeted liposomal formulations possessed similar characteristics as that of Pac-LCL. The stability evaluation showed that all these formulations were stable for three months at $5 \pm 3^{\circ} \mathrm{C}$.

In conclusion, targeted drug delivery with paclitaxel-loaded long-circulating liposomes improved the antitumor efficacy of breast cancer in a mouse xenograft model. Tumor cell targeted transferrin conjugated liposomes were more effective compared to tumor vasculature targeted cRGD conjugated liposomes. Although the effect of tumor vasculature targeted liposomes was comparable to non-targeted liposomes and paclitaxel solution, these cRGD conjugated liposomes have the potential for improving the therapeutic efficacy in tumor metastasis and primary tumors with fast developing vasculature. The stability of the paclitaxel-loaded liposomes in dispersion was improved by selecting an optimum composition. To further improve the long-term stability, these liposomes were lyophilized in presence of a non-crystallizing solute sucrose. The size, charge, and integrity of these liposomes were preserved for three months at refrigerated storage conditions. 


\section{LIST OF REFERENCES}

[1] D. Hanahan, R.A. Weinberg, The hallmarks of cancer. Cell 100(1) (2000) 57-70.

[2] M. Heron, D.L. Hoyert, S.L. Murphy, J. Xu, K.D. Kochanek, B. Tejada-Vera, Deaths: final data for 2006. Natl Vital Stat Rep 57(14) (2009) 1-134.

[3] M. Heron, Deaths: leading causes for 2006. Natl Vital Stat Rep 58(14) (2010) 1100.

[4] A. Jemal, R. Siegel, E. Ward, Y. Hao, J. Xu, M.J. Thun, Cancer statistics, 2009. CA Cancer J Clin 59(4) (2009) 225-249.

[5] S.H. Jang, M.G. Wientjes, D. Lu, J.L. Au, Drug delivery and transport to solid tumors. Pharm Res 20(9) (2003) 1337-1350.

[6] R.K. Jain, Normalization of tumor vasculature: an emerging concept in antiangiogenic therapy. Science 307(5706) (2005) 58-62.

[7] R.K. Jain, Delivery of molecular and cellular medicine to solid tumors. J Control Release 53(1-3) (1998) 49-67.

[8] E.K. Rowinsky, R.C. Donehower, Paclitaxel (taxol). N Engl J Med 332(15) (1995) 1004-1014.

[9] C.M. Spencer, D. Faulds, Paclitaxel. A review of its pharmacodynamic and pharmacokinetic properties and therapeutic potential in the treatment of cancer. Drugs 48(5) (1994) 794-847.

[10] A. Goncalves, D. Braguer, G. Carles, N. Andre, C. Prevot, C. Briand, Caspase-8 activation independent of CD95/CD95-L interaction during paclitaxel-induced apoptosis in human colon cancer cells (HT29-D4). Biochem Pharmacol 60(11) (2000) 1579-1584.

[11] M. Carre, G. Carles, N. Andre, S. Douillard, J. Ciccolini, C. Briand, D. Braguer, Involvement of microtubules and mitochondria in the antagonism of arsenic trioxide on paclitaxel-induced apoptosis. Biochem Pharmacol 63(10) (2002) 1831-1842.

[12] N. Andre, M. Carre, G. Brasseur, B. Pourroy, H. Kovacic, C. Briand, D. Braguer, Paclitaxel targets mitochondria upstream of caspase activation in intact human neuroblastoma cells. FEBS Lett 532(1-2) (2002) 256-260.

[13] G.W. Makin, B.M. Corfe, G.J. Griffiths, A. Thistlethwaite, J.A. Hickman, C. Dive, Damage-induced Bax N-terminal change, translocation to mitochondria and formation of Bax dimers/complexes occur regardless of cell fate. Embo J 20(22) (2001) 6306-6315.

[14] S. Haldar, N. Jena, C.M. Croce, Inactivation of Bcl-2 by phosphorylation. Proc Natl Acad Sci USA 92(10) (1995) 4507-4511.

[15] R.B. Weiss, R.C. Donehower, P.H. Wiernik, T. Ohnuma, R.J. Gralla, D.L. Trump, J.R. Baker, Jr., D.A. Van Echo, D.D. Von Hoff, B. Leyland-Jones, Hypersensitivity reactions from taxol. J Clin Oncol 8(7) (1990) 1263-1268.

[16] W.J. Gradishar, S. Tjulandin, N. Davidson, H. Shaw, N. Desai, P. Bhar, M. Hawkins, J. O'Shaughnessy, Phase III trial of nanoparticle albumin-bound paclitaxel compared with polyethylated castor oil-based paclitaxel in women with breast cancer. J Clin Oncol 23(31) (2005) 7794-7803. 
[17] N.I. Marupudi, J.E. Han, K.W. Li, V.M. Renard, B.M. Tyler, H. Brem, Paclitaxel: a review of adverse toxicities and novel delivery strategies. Expert Opin Drug Saf 6(5) (2007) 609-621.

[18] R.K. Jain, Transport phenomena in tumors. In: J. Wei, J. Anderson, M. Denn, J. Seinfeld, G. Stephanopoulos and J. Wei (Eds.), Advances in Chemical Engineering, Vol. 19, Academic Press Inc., San Diego, CA, 1994, pp. 129-200.

[19] R.K. Jain, Barriers to drug delivery in solid tumors. Sci Am 271(1) (1994) 58-65.

[20] J.K. Vasir, M.K. Reddy, V.D. Labhasetwar, Nanosystems in drug targeting: opportunities and challenges. Current Nanoscience 1 (2005) 47-64.

[21] E. Ruoslahti, S.N. Bhatia, M.J. Sailor, Targeting of drugs and nanoparticles to tumors. J Cell Biol 188(6) (2010) 759-768.

[22] M.E. Davis, Z.G. Chen, D.M. Shin, Nanoparticle therapeutics: an emerging treatment modality for cancer. Nat Rev Drug Discov 7(9) (2008) 771-782.

[23] V.P. Torchilin, Recent advances with liposomes as pharmaceutical carriers. Nat Rev Drug Discov 4(2) (2005) 145-160.

[24] D. Kirpotin, J.W. Park, K. Hong, S. Zalipsky, W.L. Li, P. Carter, C.C. Benz, D. Papahadjopoulos, Sterically stabilized anti-HER2 immunoliposomes: design and targeting to human breast cancer cells in vitro. Biochemistry 36(1) (1997) 66-75.

[25] A. Gabizon, D. Goren, A.T. Horowitz, D. Tzemach, A. Lossos, T. Siegal, Longcirculating liposomes for drug delivery in cancer therapy: a review of biodistribution studies in tumor-bearing animals. Adv Drug Del Rev 24(2-3) (1997) 337-344.

[26] T. Yang, M.K. Choi, F.D. Cui, S.J. Lee, S.J. Chung, C.K. Shim, D.D. Kim, Antitumor effect of paclitaxel-loaded PEGylated immunoliposomes against human breast cancer cells. Pharm Res 24(12) (2007) 2402-2411.

[27] N.Z. Wu, D. Da, T.L. Rudoll, D. Needham, A.R. Whorton, M.W. Dewhirst, Increased microvascular permeability contributes to preferential accumulation of stealth liposomes in tumor tissue. Cancer Res 53(16) (1993) 3765-3770.

[28] T. Lian, R.J. Ho, Trends and developments in liposome drug delivery systems. J Pharm Sci 90(6) (2001) 667-680.

[29] U.S. Kadimi, D.R. Balasubramanian, U.R. Ganni, M. Balaraman, V. Govindarajulu, In vitro studies on liposomal amphotericin B obtained by supercritical carbon dioxide-mediated process. Nanomedicine 3(4) (2007) 273280.

[30] Y.L. Lo, J.C. Tsai, J.H. Kuo, Liposomes and disaccharides as carriers in spraydried powder formulations of superoxide dismutase. J Control Release 94(2-3) (2004) 259-272.

[31] K. Mishima, Biodegradable particle formation for drug and gene delivery using supercritical fluid and dense gas. Adv Drug Deliv Rev 60(3) (2008) 411-432.

[32] C. Chen, D. Han, C. Cai, X. Tang, An overview of liposome lyophilization and its future potential. J Control Release 142(3) (2010) 299-311.

[33] T.D. Madden, M.B. Bally, M.J. Hope, P.R. Cullis, H.P. Schieren, A.S. Janoff, Protection of large unilamellar vesicles by trehalose during dehydration: retention of vesicle contents. Biochim Biophys Acta 817(1) (1985) 67-74.

[34] E.C. van Winden, Freeze-drying of liposomes: theory and practice. Methods Enzymol 367 (2003) 99-110. 
[35] S.L. Huang, R.C. MacDonald, Acoustically active liposomes for drug encapsulation and ultrasound-triggered release. Biochim Biophys Acta 1665(1-2) (2004) 134-141.

[36] S.M. Stieger, C.F. Caskey, R.H. Adamson, S. Qin, F.R. Curry, E.R. Wisner, K.W. Ferrara, Enhancement of vascular permeability with low-frequency contrastenhanced ultrasound in the chorioallantoic membrane model. Radiology 243(1) (2007) 112-121.

[37] E.C. Unger, T. Porter, W. Culp, R. Labell, T. Matsunaga, R. Zutshi, Therapeutic applications of lipid-coated microbubbles. Adv Drug Deliv Rev 56(9) (2004) 1291-1314.

[38] W.G. Pitt, G.A. Husseini, B.J. Staples, Ultrasonic drug delivery - a general review. Expert Opin Drug Deliv 1(1) (2004) 37-56.

[39] A.L. Klibanov, Microbubble contrast agents: targeted ultrasound imaging and ultrasound-assisted drug-delivery applications. Invest Radiol 41(3) (2006) 354362.

[40] A.D. Bangham, R.W. Horne, Negative staining of phospholipids and their structural modification by surface-active agents as observed in the electron microscope. J Mol Biol 8 (1964) 660-668.

[41] A.D. Bangham, M.M. Standish, J.C. Watkins, Diffusion of univalent ions across the lamellae of swollen phospholipids. J Mol Biol 13(1) (1965) 238-252.

[42] A.D. Bangham, M.M. Standish, G. Weissmann, The action of steroids and streptolysin S on the permeability of phospholipid structures to cations. J Mol Biol 13(1) (1965) 253-259.

[43] A.D. Bangham, Liposomes: the Babraham connection. Chem Phys Lipids 64(1-3) (1993) 275-285.

[44] G. Sessa, G. Weissmann, Phospholipid spherules (liposomes) as a model for biological membranes. J Lipid Res 9(3) (1968) 310-318.

[45] G. Gregoriadis, P.D. Leathwood, B.E. Ryman, Enzyme entrapment in liposomes. FEBS Lett 14(2) (1971) 95-99.

[46] G. Gregoriadis, B.E. Ryman, Liposomes as carriers of enzymes or drugs: a new approach to the treatment of storage diseases. Biochem J 124(5) (1971) 58P.

[47] G. Gregoriadis, B.E. Ryman, Lysosomal localization of enzyme-containing liposomes injected into rats. Biochem J 128(4) (1972) 142P-143P.

[48] G. Gregoriadis, B.E. Ryman, Lysosomal localization of fructofuranosidase containing liposomes injected into rats. Biochem J 129(1) (1972) 123-133.

[49] G. Gregoriadis, Drug entrapment in liposomes. FEBS Lett 36(3) (1973) 292-296.

[50] G. Gregoriadis, E.J. Wills, C.P. Swain, A.S. Tavill, Drug-carrier potential of liposomes in cancer chemotherapy. Lancet 1(7870) (1974) 1313-1316.

[51] T. Kaasgaard, T.L. Andresen, Liposomal cancer therapy: exploiting tumor characteristics. Expert Opin Drug Deliv 7(2) (2010) 225-243.

[52] V.P. Torchilin, Fluorescence microscopy to follow the targeting of liposomes and micelles to cells and their intracellular fate. Adv Drug Deliv Rev 57(1) (2005) 95109.

[53] D.C. Drummond, O. Meyer, K. Hong, D.B. Kirpotin, D. Papahadjopoulos, Optimizing liposomes for delivery of chemotherapeutic agents to solid tumors. Pharmacol Rev 51(4) (1999) 691-743. 
[54] N. Maurer, D.B. Fenske, P.R. Cullis, Developments in liposomal drug delivery systems. Expert Opin Biol Ther 1(6) (2001) 923-947.

[55] J. de Gier, J.G. Mandersloot, L.L. van Deenen, Lipid composition and permeability of liposomes. Biochim Biophys Acta 150(4) (1968) 666-675.

[56] T.M. Koyama, C.R. Stevens, E.J. Borda, K.J. Grobe, D.A. Cleary, Characterizing the gel to liquid crystal transition in lipid-bilayer model systems. Chem. Educator 4(1) (1999) 12-15.

[57] J. Risbo, K. Jorgensen, M.M. Sperotto, O.G. Mouritsen, Phase behavior and permeability properties of phospholipid bilayers containing a short-chain phospholipid permeability enhancer. Biochim Biophys Acta 1329(1) (1997) 8596.

[58] D.O. Shah, J.H. Schulman, Influence of calcium, cholesterol, and unsaturation on lecithin monolayers. J Lipid Res 8(3) (1967) 215-226.

[59] B.D. Ladbrooke, R.M. Williams, D. Chapman, Studies on lecithin-cholesterolwater interactions by differential scanning calorimetry and X-ray diffraction. Biochim Biophys Acta 150(3) (1968) 333-340.

[60] D. Papahadjopoulos, J.C. Watkins, Phospholipid model membranes. II. Permeability properties of hydrated liquid crystals. Biochim Biophys Acta 135(4) (1967) 639-652.

[61] J.d. Gier, C.W.M. Haest, J.G. Mandersloot, L.L.M.v. Deenen, Valinomycininduced permeation of ${ }^{86} \mathrm{Rb}^{+}$of liposomes with varying composition through the bilayers. Biochim Biophys Acta 211 (1970) 373-375.

[62] R.A. Demel, K.R. Bruckdorfer, L.L. van Deenen, The effect of sterol structure on the permeability of lipomes to glucose, glycerol and $\mathrm{Rb}^{+}$. Biochim Biophys Acta 255(1) (1972) 321-330.

[63] R.A. Demel, B. De Kruyff, The function of sterols in membranes. Biochim Biophys Acta 457(2) (1976) 109-132.

[64] C. Kirby, J. Clarke, G. Gregoriadis, Effect of the cholesterol content of small unilamellar liposomes on their stability in vivo and in vitro. Biochem J 186(2) (1980) 591-598.

[65] J. Senior, J.C. Crawley, G. Gregoriadis, Tissue distribution of liposomes exhibiting long half-lives in the circulation after intravenous injection. Biochim Biophys Acta 839(1) (1985) 1-8.

[66] I. Abraham, A. Goundalkar, M. Mezei, Effect of liposomal surface charge on the pharmacokinetics of an encapsulated model compound. Biopharm Drug Dispos 5(4) (1984) 387-398.

[67] A.M. Abraham, A. Walubo, The effect of surface charge on the disposition of liposome-encapsulated gentamicin to the rat liver, brain, lungs and kidneys after intraperitoneal administration. Int J Antimicrob Agents 25(5) (2005) 392-397.

[68] C.D. Black, G. Gregoriadis, Interaction of liposomes with blood plasma proteins. Biochem Soc Trans 4(2) (1976) 253-256.

[69] D.J. Wilkins, P.A. Myers, Studies on the relationship between the electrophoretic properties of colloids and their blood clearance and organ distribution in the rat. Br J Exp Pathol 47(6) (1966) 568-576. 
[70] F. Roerdink, N.M. Wassef, E.C. Richardson, C.R. Alving, Effects of negatively charged lipids on phagocytosis of liposomes opsonized by complement. Biochim Biophys Acta 734(1) (1983) 33-39.

[71] T. Ogiso, T. Yamaguchi, M. Iwaki, T. Tanino, Y. Miyake, Effect of positively and negatively charged liposomes on skin permeation of drugs. J Drug Target 9(1) (2001) 49-59.

[72] D. Baczynska, K. Widerak, M. Ugorski, M. Langner, Surface charge and the association of liposomes with colon carcinoma cells. Z Naturforsch C 56(9-10) (2001) 872-877.

[73] T.D. Heath, N.G. Lopez, D. Papahadjopoulos, The effects of liposome size and surface charge on liposome-mediated delivery of methotrexate-gamma-aspartate to cells in vitro. Biochim Biophys Acta 820(1) (1985) 74-84.

[74] R.A. Schwendener, P.A. Lagocki, Y.E. Rahman, The effects of charge and size on the interaction of unilamellar liposomes with macrophages. Biochim Biophys Acta 772(1) (1984) 93-101.

[75] W.G. Love, N. Amos, B.D. Williams, I.W. Kellaway, Effect of liposome surface charge on the stability of technetium $\left({ }^{99} \mathrm{mTc}\right)$ radiolabelled liposomes. J Microencapsul 6(1) (1989) 105-113.

[76] C.R. Miller, B. Bondurant, S.D. McLean, K.A. McGovern, D.F. O'Brien, Liposome-cell interactions in vitro: effect of liposome surface charge on the binding and endocytosis of conventional and sterically stabilized liposomes. Biochemistry 37(37) (1998) 12875-12883.

[77] S. Krasnici, A. Werner, M.E. Eichhorn, M. Schmitt-Sody, S.A. Pahernik, B. Sauer, B. Schulze, M. Teifel, U. Michaelis, K. Naujoks, M. Dellian, Effect of the surface charge of liposomes on their uptake by angiogenic tumor vessels. Int J Cancer 105(4) (2003) 561-567.

[78] M. Schmitt-Sody, S. Krasnici, P.C. Manegold, B. Sauer, B. Schulze, M. Teifel, U. Michaelis, M. Dellian, Paclitaxel encapsulated in cationic liposomes: effect on growth and metastases of solid tumors in vivo. Chirurgisches Forum 2001 für experimentelle und klinische Forschung, Springer, Heidelberg, 2001, pp. 61-63.

[79] H. Harashima, H. Kiwada, Liposomal targeting and drug delivery: kinetic consideration. Adv Drug Del Rev 19 (1996) 425-444.

[80] T.M. Allen, C. Hansen, J. Rutledge, Liposomes with prolonged circulation times: factors affecting uptake by reticuloendothelial and other tissues. Biochim Biophys Acta 981(1) (1989) 27-35.

[81] H. Harashima, K. Sakata, K. Funato, H. Kiwada, Enhanced hepatic uptake of liposomes through complement activation depending on the size of liposomes. Pharm Res 11(3) (1994) 402-406.

[82] T.M. Allen, J.M. Everest, Effect of liposome size and drug release properties on pharmacokinetics of encapsulated drug in rats. J Pharmacol Exp Ther 226(2) (1983) 539-544.

[83] Y.E. Rahman, E.A. Cerny, K.R. Patel, E.H. Lau, B.J. Wright, Differential uptake of liposomes varying in size and lipid composition by parenchymal and kupffer cells of mouse liver. Life Sci 31(19) (1982) 2061-2071.

[84] R.M. Abra, C.A. Hunt, Liposome disposition in vivo. III. Dose and vesicle-size effects. Biochim Biophys Acta 666(3) (1981) 493-503. 
[85] T.M. Allen, A. Chonn, Large unilamellar liposomes with low uptake into the reticuloendothelial system. FEBS Lett 223(1) (1987) 42-46.

[86] A. Gabizon, D. Papahadjopoulos, Liposome formulations with prolonged circulation time in blood and enhanced uptake by tumors. Proc Natl Acad Sci USA 85(18) (1988) 6949-6953.

[87] A. Gabizon, D. Papahadjopoulos, The role of surface charge and hydrophilic groups on liposome clearance in vivo. Biochim Biophys Acta 1103(1) (1992) 94100.

[88] G. Blume, G. Cevc, Liposomes for the sustained drug release in vivo. Biochim Biophys Acta 1029(1) (1990) 91-97.

[89] A.L. Klibanov, K. Maruyama, V.P. Torchilin, L. Huang, Amphipathic polyethyleneglycols effectively prolong the circulation time of liposomes. FEBS Lett 268(1) (1990) 235-237.

[90] T.S. Levchenko, R. Rammohan, A.N. Lukyanov, K.R. Whiteman, V.P. Torchilin, Liposome clearance in mice: the effect of a separate and combined presence of surface charge and polymer coating. Int J Pharm 240(1-2) (2002) 95-102.

[91] G.N. Chiu, M.B. Bally, L.D. Mayer, Selective protein interactions with phosphatidylserine containing liposomes alter the steric stabilization properties of poly(ethylene glycol). Biochim Biophys Acta 1510(1-2) (2001) 56-69.

[92] H. Takeuchi, H. Yamamoto, T. Toyoda, H. Toyobuku, T. Hino, Y. Kawashima, Physical stability of size controlled small unilameller liposomes coated with a modified polyvinyl alcohol. Int J Pharm 164(1-2) (1998) 103-111.

[93] K. Nakano, Y. Tozuka, H. Takeuchi, Effect of surface properties of liposomes coated with a modified polyvinyl alcohol (PVA-R) on the interaction with macrophage cells. Int J Pharm 354(1-2) (2008) 174-179.

[94] T. Shehata, K. Ogawara, K. Higaki, T. Kimura, Prolongation of residence time of liposome by surface-modification with mixture of hydrophilic polymers. Int $\mathbf{J}$ Pharm 359(1-2) (2008) 272-279.

[95] L.D. Mayer, M.B. Bally, M.J. Hope, P.R. Cullis, Techniques for encapsulating bioactive agents into liposomes. Chem Phys Lipids 40(2-4) (1986) 333-345.

[96] C.M. Colley, B.E. Ryman, Liposomes as carriers in vivo for methotrexate. Biochem Soc Trans 3(1) (1975) 157-159.

[97] L.D. Mayer, M.B. Bally, M.J. Hope, P.R. Cullis, Uptake of antineoplastic agents into large unilamellar vesicles in response to a membrane potential. Biochim Biophys Acta 816(2) (1985) 294-302.

[98] F. Szoka, Jr., D. Papahadjopoulos, Procedure for preparation of liposomes with large internal aqueous space and high capture by reverse-phase evaporation. Proc Natl Acad Sci USA 75(9) (1978) 4194-4198.

[99] E. Goormaghtigh, P. Chatelain, J. Caspers, J.M. Ruysschaert, Evidence of a specific complex between adriamycin and negatively-charged phospholipids. Biochim Biophys Acta 597(1) (1980) 1-14.

[100] A. Gabizon, A. Dagan, D. Goren, Y. Barenholz, Z. Fuks, Liposomes as in vivo carriers of adriamycin: reduced cardiac uptake and preserved antitumor activity in mice. Cancer Res 42(11) (1982) 4734-4739. 
[101] A. Rahman, G. White, N. More, P.S. Schein, Pharmacological, toxicological, and therapeutic evaluation in mice of doxorubicin entrapped in cardiolipin liposomes. Cancer Res 45(2) (1985) 796-803.

[102] L.D. Mayer, M.B. Bally, P.R. Cullis, Uptake of adriamycin into large unilamellar vesicles in response to a pH gradient. Biochim Biophys Acta 857(1) (1986) 123126.

[103] G. Haran, R. Cohen, L.K. Bar, Y. Barenholz, Transmembrane ammonium sulfate gradients in liposomes produce efficient and stable entrapment of amphipathic weak bases. Biochim Biophys Acta 1151(2) (1993) 201-215.

[104] P.G. Tardi, R.C. Gallagher, S. Johnstone, N. Harasym, M. Webb, M.B. Bally, L.D. Mayer, Coencapsulation of irinotecan and floxuridine into low cholesterolcontaining liposomes that coordinate drug release in vivo. Biochim Biophys Acta 1768(3) (2007) 678-687.

[105] Y.F. Zhang, J.C. Wang, D.Y. Bian, X. Zhang, Q. Zhang, Targeted delivery of RGD-modified liposomes encapsulating both combretastatin A-4 and doxorubicin for tumor therapy: in vitro and in vivo studies. Eur J Pharm Biopharm 74(3) (2010) 467-473.

[106] X. Li, G.R. Ruan, W.L. Lu, H.Y. Hong, G.W. Liang, Y.T. Zhang, Y. Liu, C. Long, X. Ma, L. Yuan, J.C. Wang, X. Zhang, Q. Zhang, A novel stealth liposomal topotecan with amlodipine: apoptotic effect is associated with deletion of intracellular $\mathrm{Ca}^{2+}$ by amlodipine thus leading to an enhanced antitumor activity in leukemia. J Control Release 112(2) (2006) 186-198.

[107] M.C. Garnett, Targeted drug conjugates: principles and progress. Adv Drug Deliv Rev 53(2) (2001) 171-216.

[108] L.E. Gerlowski, R.K. Jain, Microvascular permeability of normal and neoplastic tissues. Microvasc Res 31(3) (1986) 288-305.

[109] H.F. Dvorak, J.A. Nagy, J.T. Dvorak, A.M. Dvorak, Identification and characterization of the blood vessels of solid tumors that are leaky to circulating macromolecules. Am J Pathol 133(1) (1988) 95-109.

[110] R.K. Jain, Transport of molecules across tumor vasculature. Cancer Metastasis Rev 6(4) (1987) 559-593.

[111] Y. Matsumura, H. Maeda, A new concept for macromolecular therapeutics in cancer chemotherapy: mechanism of tumoritropic accumulation of proteins and the antitumor agent smancs. Cancer Res 46(12 Pt 1) (1986) 6387-6392.

[112] H. Maeda, The enhanced permeability and retention (EPR) effect in tumor vasculature: the key role of tumor-selective macromolecular drug targeting. Adv Enzyme Regul 41 (2001) 189-207.

[113] H. Maeda, J. Wu, T. Sawa, Y. Matsumura, K. Hori, Tumor vascular permeability and the EPR effect in macromolecular therapeutics: a review. J Control Release 65(1-2) (2000) 271-284.

[114] F. Yuan, M. Dellian, D. Fukumura, M. Leunig, D.A. Berk, V.P. Torchilin, R.K. Jain, Vascular permeability in a human tumor xenograft: molecular size dependence and cutoff size. Cancer Res 55(17) (1995) 3752-3756.

[115] S.K. Hobbs, W.L. Monsky, F. Yuan, W.G. Roberts, L. Griffith, V.P. Torchilin, R.K. Jain, Regulation of transport pathways in tumor vessels: role of tumor type and microenvironment. Proc Natl Acad Sci USA 95(8) (1998) 4607-4612. 
[116] Y. Nishioka, H. Yoshino, Lymphatic targeting with nanoparticulate system. Adv Drug Deliv Rev 47(1) (2001) 55-64.

[117] H. Hatakeyama, H. Akita, E. Ishida, K. Hashimoto, H. Kobayashi, T. Aoki, J. Yasuda, K. Obata, H. Kikuchi, T. Ishida, H. Kiwada, H. Harashima, Tumor targeting of doxorubicin by anti-MT1-MMP antibody-modified PEG liposomes. Int J Pharm 342(1-2) (2007) 194-200.

[118] D.B. Kirpotin, D.C. Drummond, Y. Shao, M.R. Shalaby, K. Hong, U.B. Nielsen, J.D. Marks, C.C. Benz, J.W. Park, Antibody targeting of long-circulating lipidic nanoparticles does not increase tumor localization but does increase internalization in animal models. Cancer Res 66(13) (2006) 6732-6740.

[119] M.H. Kranenborg, O.C. Boerman, J.C. Oosterwijk-Wakka, M.C. de Weijert, F.H. Corstens, E. Oosterwijk, Two-step radio-immunotargeting of renal-cell carcinoma xenografts in nude mice with anti-renal-cell-carcinoma $\mathrm{X}$ anti-DTPA bispecific monoclonal antibodies. Int J Cancer 75(1) (1998) 74-80.

[120] V.K. Jansons, P.L. Mallett, Targeted liposomes: a method for preparation and analysis. Anal Biochem 111(1) (1981) 54-59.

[121] G. Gregoriadis, E.D. Neerunjun, Homing of liposomes to target cells. Biochem Biophys Res Commun 65(2) (1975) 537-544.

[122] G. Gregoriadis, The carrier potential of liposomes in biology and medicine (second of two parts). N Engl J Med 295(14) (1976) 765-770.

[123] G. Blume, G. Cevc, M.D. Crommelin, I.A. Bakker-Woudenberg, C. Kluft, G. Storm, Specific targeting with poly(ethylene glycol)-modified liposomes: coupling of homing devices to the ends of the polymeric chains combines effective target binding with long circulation times. Biochim Biophys Acta 1149(1) (1993) 180-184.

[124] T.M. Allen, E. Brandeis, C.B. Hansen, G.Y. Kao, S. Zalipsky, A new strategy for attachment of antibodies to sterically stabilized liposomes resulting in efficient targeting to cancer cells. Biochim Biophys Acta 1237(2) (1995) 99-108.

[125] K. Maruyama, N. Takahashi, T. Tagawa, K. Nagaike, M. Iwatsuru, Immunoliposomes bearing polyethyleneglycol-coupled Fab' fragment show prolonged circulation time and high extravasation into targeted solid tumors in vivo. FEBS Lett 413(1) (1997) 177-180.

[126] J.W. Park, D.B. Kirpotin, K. Hong, R. Shalaby, Y. Shao, U.B. Nielsen, J.D. Marks, D. Papahadjopoulos, C.C. Benz, Tumor targeting using anti-her2 immunoliposomes. J Control Release 74(1-3) (2001) 95-113.

[127] J.W. Park, K. Hong, D.B. Kirpotin, G. Colbern, R. Shalaby, J. Baselga, Y. Shao, U.B. Nielsen, J.D. Marks, D. Moore, D. Papahadjopoulos, C.C. Benz, Anti-HER2 immunoliposomes: enhanced efficacy attributable to targeted delivery. Clin Cancer Res 8(4) (2002) 1172-1181.

[128] J.N. Moreira, T. Ishida, R. Gaspar, T.M. Allen, Use of the post-insertion technique to insert peptide ligands into pre-formed stealth liposomes with retention of binding activity and cytotoxicity. Pharm Res 19(3) (2002) 265-269.

[129] S. Zalipsky, N. Mullah, J.A. Harding, J. Gittelman, L. Guo, S.A. DeFrees, Poly(ethylene glycol)-grafted liposomes with oligopeptide or oligosaccharide ligands appended to the termini of the polymer chains. Bioconjug Chem 8(2) (1997) 111-118. 
[130] P.S. Uster, T.M. Allen, B.E. Daniel, C.J. Mendez, M.S. Newman, G.Z. Zhu, Insertion of poly(ethylene glycol) derivatized phospholipid into pre-formed liposomes results in prolonged in vivo circulation time. FEBS Lett 386(2-3) (1996) 243-246.

[131] T. Ishida, D.L. Iden, T.M. Allen, A combinatorial approach to producing sterically stabilized (Stealth) immunoliposomal drugs. FEBS Lett 460(1) (1999) 129-133.

[132] Y. Lu, P.S. Low, Folate-mediated delivery of macromolecular anticancer therapeutic agents. Adv Drug Deliv Rev 54(5) (2002) 675-693.

[133] B.A. Kamen, A. Capdevila, Receptor-mediated folate accumulation is regulated by the cellular folate content. Proc Natl Acad Sci USA 83(16) (1986) 5983-5987.

[134] G. Toffoli, C. Cernigoi, A. Russo, A. Gallo, M. Bagnoli, M. Boiocchi, Overexpression of folate binding protein in ovarian cancers. Int J Cancer 74(2) (1997) 193-198.

[135] R.J. Lee, P.S. Low, Delivery of liposomes into cultured KB cells via folate receptor-mediated endocytosis. J Biol Chem 269(5) (1994) 3198-3204.

[136] R.J. Lee, P.S. Low, Folate-mediated tumor cell targeting of liposome-entrapped doxorubicin in vitro. Biochim Biophys Acta 1233(2) (1995) 134-144.

[137] D. Goren, A.T. Horowitz, D. Tzemach, M. Tarshish, S. Zalipsky, A. Gabizon, Nuclear delivery of doxorubicin via folate-targeted liposomes with bypass of multidrug-resistance efflux pump. Clin Cancer Res 6(5) (2000) 1949-1957.

[138] D.R. Richardson, D.S. Kalinowski, S. Lau, P.J. Jansson, D.B. Lovejoy, Cancer cell iron metabolism and the development of potent iron chelators as anti-tumour agents. Biochim Biophys Acta 1790(7) (2009) 702-717.

[139] D.S. Kalinowski, D.R. Richardson, The evolution of iron chelators for the treatment of iron overload disease and cancer. Pharmacol Rev 57(4) (2005) 547583.

[140] D.R. Richardson, Mobilization of iron from neoplastic cells by some iron chelators is an energy-dependent process. Biochim Biophys Acta 1320(1) (1997) 45-57.

[141] T.R. Daniels, T. Delgado, J.A. Rodriguez, G. Helguera, M.L. Penichet, The transferrin receptor part I: Biology and targeting with cytotoxic antibodies for the treatment of cancer. Clin Immunol 121(2) (2006) 144-158.

[142] T.R. Daniels, T. Delgado, G. Helguera, M.L. Penichet, The transferrin receptor part II: targeted delivery of therapeutic agents into cancer cells. Clin Immunol 121(2) (2006) 159-176.

[143] D.A. Eavarone, X. Yu, R.V. Bellamkonda, Targeted drug delivery to C6 glioma by transferrin-coupled liposomes. J Biomed Mater Res 51(1) (2000) 10-14.

[144] S. Anabousi, M. Laue, C.M. Lehr, U. Bakowsky, C. Ehrhardt, Assessing transferrin modification of liposomes by atomic force microscopy and transmission electron microscopy. Eur J Pharm Biopharm 60(2) (2005) 295-303.

[145] C. Fonseca, J.N. Moreira, C.J. Ciudad, M.C. Pedroso de Lima, S. Simoes, Targeting of sterically stabilised $\mathrm{pH}$-sensitive liposomes to human T-leukaemia cells. Eur J Pharm Biopharm 59(2) (2005) 359-366. 
[146] T. Kakudo, S. Chaki, S. Futaki, I. Nakase, K. Akaji, T. Kawakami, K. Maruyama, H. Kamiya, H. Harashima, Transferrin-modified liposomes equipped with a $\mathrm{pH}-$ sensitive fusogenic peptide: an artificial viral-like delivery system. Biochemistry 43(19) (2004) 5618-5628.

[147] O. Ishida, K. Maruyama, H. Tanahashi, M. Iwatsuru, K. Sasaki, M. Eriguchi, H. Yanagie, Liposomes bearing polyethyleneglycol-coupled transferrin with intracellular targeting property to the solid tumors in vivo. Pharm Res 18(7) (2001) 1042-1048.

[148] X. Li, L. Ding, Y. Xu, Y. Wang, Q. Ping, Targeted delivery of doxorubicin using stealth liposomes modified with transferrin. Int J Pharm 373(1-2) (2009) 116-123.

[149] H. Iinuma, K. Maruyama, K. Okinaga, K. Sasaki, T. Sekine, O. Ishida, N. Ogiwara, K. Johkura, Y. Yonemura, Intracellular targeting therapy of cisplatinencapsulated transferrin-polyethylene glycol liposome on peritoneal dissemination of gastric cancer. Int J Cancer 99(1) (2002) 130-137.

[150] R. Suzuki, T. Takizawa, Y. Kuwata, M. Mutoh, N. Ishiguro, N. Utoguchi, A. Shinohara, M. Eriguchi, H. Yanagie, K. Maruyama, Effective anti-tumor activity of oxaliplatin encapsulated in transferrin-PEG-liposome. Int J Pharm 346(1-2) (2008) 143-150.

[151] K. Maruyama, O. Ishida, S. Kasaoka, T. Takizawa, N. Utoguchi, A. Shinohara, M. Chiba, H. Kobayashi, M. Eriguchi, H. Yanagie, Intracellular targeting of sodium mercaptoundecahydrododecaborate (BSH) to solid tumors by transferrinPEG liposomes, for boron neutron-capture therapy (BNCT). J Control Release 98(2) (2004) 195-207.

[152] S. Masunaga, S. Kasaoka, K. Maruyama, D. Nigg, Y. Sakurai, K. Nagata, M. Suzuki, Y. Kinashi, A. Maruhashi, K. Ono, The potential of transferrin-pendanttype polyethyleneglycol liposomes encapsulating decahydrodecaborate-(10)B (GB-10) as (10)B-carriers for boron neutron capture therapy. Int J Radiat Oncol Biol Phys 66(5) (2006) 1515-1522.

[153] K.M. Hege, D.L. Daleke, T.A. Waldmann, K.K. Matthay, Comparison of anti-Tac and anti-transferrin receptor-conjugated liposomes for specific drug delivery to adult T-cell leukemia. Blood 74(6) (1989) 2043-2052.

[154] T. Kobayashi, T. Ishida, Y. Okada, S. Ise, H. Harashima, H. Kiwada, Effect of transferrin receptor-targeted liposomal doxorubicin in P-glycoprotein-mediated drug resistant tumor cells. Int J Pharm 329(1-2) (2007) 94-102.

[155] M.L. Krieger, N. Eckstein, V. Schneider, M. Koch, H.D. Royer, U. Jaehde, G. Bendas, Overcoming cisplatin resistance of ovarian cancer cells by targeted liposomes in vitro. Int J Pharm 389(1-2) (2010) 10-17.

[156] J. Wu, Y. Lu, A. Lee, X. Pan, X. Yang, X. Zhao, R.J. Lee, Reversal of multidrug resistance by transferrin-conjugated liposomes co-encapsulating doxorubicin and verapamil. J Pharm Pharm Sci 10(3) (2007) 350-357.

[157] V. Soni, D.V. Kohli, S.K. Jain, Transferrin coupled liposomes as drug delivery carriers for brain targeting of 5-florouracil. J Drug Target 13(4) (2005) 245-250.

[158] V. Soni, D.V. Kohli, S.K. Jain, Transferrin-conjugated liposomal system for improved delivery of 5-fluorouracil to brain. J Drug Target 16(1) (2008) 73-78. 
[159] M.K. Divi, Development and evaluation of brain tumor targeted liposome delivery system for paclitaxel. [Dissertation], [Memphis], The University of Tennessee Health Science Center, (2007), 126 p.

[160] T.W. Hambley, W.N. Hait, Is anticancer drug development heading in the right direction? Cancer Res 69(4) (2009) 1259-1262.

[161] D. Hanahan, J. Folkman, Patterns and emerging mechanisms of the angiogenic switch during tumorigenesis. Cell 86(3) (1996) 353-364.

[162] J. Folkman, Addressing tumor blood vessels. Nat Biotechnol 15(6) (1997) 510.

[163] W. Arap, R. Pasqualini, E. Ruoslahti, Cancer treatment by targeted drug delivery to tumor vasculature in a mouse model. Science 279(5349) (1998) 377-380.

[164] R. Pasqualini, E. Koivunen, E. Ruoslahti, Alpha v integrins as receptors for tumor targeting by circulating ligands. Nat Biotechnol 15(6) (1997) 542-546.

[165] A.P. Janssen, R.M. Schiffelers, T.L. ten Hagen, G.A. Koning, A.J. Schraa, R.J. Kok, G. Storm, G. Molema, Peptide-targeted PEG-liposomes in anti-angiogenic therapy. Int J Pharm 254(1) (2003) 55-58.

[166] R.M. Schiffelers, G.A. Koning, T.L. ten Hagen, M.H. Fens, A.J. Schraa, A.P. Janssen, R.J. Kok, G. Molema, G. Storm, Anti-tumor efficacy of tumor vasculature-targeted liposomal doxorubicin. J Control Release 91(1-2) (2003) $115-122$.

[167] P. Holig, M. Bach, T. Volkel, T. Nahde, S. Hoffmann, R. Muller, R.E. Kontermann, Novel RGD lipopeptides for the targeting of liposomes to integrinexpressing endothelial and melanoma cells. Protein Eng Des Sel 17(5) (2004) 433-441.

[168] X.B. Xiong, Y. Huang, W.L. Lu, X. Zhang, H. Zhang, T. Nagai, Q. Zhang, Intracellular delivery of doxorubicin with RGD-modified sterically stabilized liposomes for an improved antitumor efficacy: in vitro and in vivo. J Pharm Sci 94(8) (2005) 1782-1793.

[169] X.B. Xiong, Y. Huang, W.L. Lu, H. Zhang, X. Zhang, Q. Zhang, Enhanced intracellular uptake of sterically stabilized liposomal Doxorubicin in vitro resulting in improved antitumor activity in vivo. Pharm Res 22(6) (2005) 933-939.

[170] X.B. Xiong, Y. Huang, W.L. Lu, X. Zhang, H. Zhang, T. Nagai, Q. Zhang, Enhanced intracellular delivery and improved antitumor efficacy of doxorubicin by sterically stabilized liposomes modified with a synthetic RGD mimetic. J Control Release 107(2) (2005) 262-275.

[171] H. Zhao, J.C. Wang, Q.S. Sun, C.L. Luo, Q. Zhang, RGD-based strategies for improving antitumor activity of paclitaxel-loaded liposomes in nude mice xenografted with human ovarian cancer. J Drug Target 17(1) (2009) 10-18.

[172] C.B. Pattillo, F. Sari-Sarraf, R. Nallamothu, B.M. Moore, G.C. Wood, M.F. Kiani, Targeting of the antivascular drug combretastatin to irradiated tumors results in tumor growth delay. Pharm Res 22(7) (2005) 1117-1120.

[173] P.K. Dubey, V. Mishra, S. Jain, S. Mahor, S.P. Vyas, Liposomes modified with cyclic RGD peptide for tumor targeting. J Drug Target 12(5) (2004) 257-264.

[174] E.A. Murphy, B.K. Majeti, L.A. Barnes, M. Makale, S.M. Weis, K. Lutu-Fuga, W. Wrasidlo, D.A. Cheresh, Nanoparticle-mediated drug delivery to tumor vasculature suppresses metastasis. Proc Natl Acad Sci USA 105(27) (2008) 93439348. 
[175] F. Pastorino, C. Brignole, D. Marimpietri, M. Cilli, C. Gambini, D. Ribatti, R. Longhi, T.M. Allen, A. Corti, M. Ponzoni, Vascular damage and anti-angiogenic effects of tumor vessel-targeted liposomal chemotherapy. Cancer Res 63(21) (2003) 7400-7409.

[176] S.V. Garde, A.J. Forte, M. Ge, E.A. Lepekhin, C.J. Panchal, S.A. Rabbani, J.J. $\mathrm{Wu}$, Binding and internalization of NGR-peptide-targeted liposomal doxorubicin (TVT-DOX) in CD13-expressing cells and its antitumor effects. Anticancer Drugs 18(10) (2007) 1189-1200.

[177] M. Loi, S. Marchio, P. Becherini, D. Di Paolo, M. Soster, F. Curnis, C. Brignole, G. Pagnan, P. Perri, I. Caffa, R. Longhi, B. Nico, F. Bussolino, C. Gambini, D. Ribatti, M. Cilli, W. Arap, R. Pasqualini, T.M. Allen, A. Corti, M. Ponzoni, F. Pastorino, Combined targeting of perivascular and endothelial tumor cells enhances anti-tumor efficacy of liposomal chemotherapy in neuroblastoma. $\mathrm{J}$ Control Release 145(1) (2010) 66-73.

[178] G.M. Kuesters, R.B. Campbell, Conjugation of bevacizumab to cationic liposomes enhances their tumor-targeting potential. Nanomedicine (Lond) 5(2) (2010) 181-192.

[179] F. Pastorino, C. Brignole, D. Di Paolo, B. Nico, A. Pezzolo, D. Marimpietri, G. Pagnan, F. Piccardi, M. Cilli, R. Longhi, D. Ribatti, A. Corti, T.M. Allen, M. Ponzoni, Targeting liposomal chemotherapy via both tumor cell-specific and tumor vasculature-specific ligands potentiates therapeutic efficacy. Cancer Res 66(20) (2006) 10073-10082.

[180] J.M. Saul, A.V. Annapragada, R.V. Bellamkonda, A dual-ligand approach for enhancing targeting selectivity of therapeutic nanocarriers. J Control Release 114(3) (2006) 277-287.

[181] X. Ying, H. Wen, W.L. Lu, J. Du, J. Guo, W. Tian, Y. Men, Y. Zhang, R.J. Li, T.Y. Yang, D.W. Shang, J.N. Lou, L.R. Zhang, Q. Zhang, Dual-targeting daunorubicin liposomes improve the therapeutic efficacy of brain glioma in animals. J Control Release 141(2) (2010) 183-192.

[182] C.H. Chen, D.Z. Liu, H.W. Fang, H.J. Liang, T.S. Yang, S.Y. Lin, Evaluation of multi-target and single-target liposomal drugs for the treatment of gastric cancer. Biosci Biotechnol Biochem 72(6) (2008) 1586-1594.

[183] V.P. Torchilin, Cell penetrating peptide-modified pharmaceutical nanocarriers for intracellular drug and gene delivery. Biopolymers 90(5) (2008) 604-610.

[184] V.P. Torchilin, R. Rammohan, V. Weissig, T.S. Levchenko, TAT peptide on the surface of liposomes affords their efficient intracellular delivery even at low temperature and in the presence of metabolic inhibitors. Proc Natl Acad Sci USA 98(15) (2001) 8786-8791.

[185] Y.L. Tseng, J.J. Liu, R.L. Hong, Translocation of liposomes into cancer cells by cell-penetrating peptides penetratin and tat: a kinetic and efficacy study. Mol Pharmacol 62(4) (2002) 864-872.

[186] R.M. Sawant, J.P. Hurley, S. Salmaso, A. Kale, E. Tolcheva, T.S. Levchenko, V.P. Torchilin, "SMART" drug delivery systems: double-targeted $\mathrm{pH}$-responsive pharmaceutical nanocarriers. Bioconjug Chem 17(4) (2006) 943-949. 
[187] C. Marty, C. Meylan, H. Schott, K. Ballmer-Hofer, R.A. Schwendener, Enhanced heparan sulfate proteoglycan-mediated uptake of cell-penetrating peptidemodified liposomes. Cell Mol Life Sci 61(14) (2004) 1785-1794.

[188] D.C. Drummond, M. Zignani, J. Leroux, Current status of pH-sensitive liposomes in drug delivery. Prog Lipid Res 39(5) (2000) 409-460.

[189] H. Karanth, R.S. Murthy, pH-sensitive liposomes - principle and application in cancer therapy. J Pharm Pharmacol 59(4) (2007) 469-483.

[190] I.Y. Kim, Y.S. Kang, D.S. Lee, H.J. Park, E.K. Choi, Y.K. Oh, H.J. Son, J.S. Kim, Antitumor activity of EGFR targeted $\mathrm{pH}$-sensitive immunoliposomes encapsulating gemcitabine in A549 xenograft nude mice. J Control Release 140(1) (2009) 55-60.

[191] T. Ishida, M.J. Kirchmeier, E.H. Moase, S. Zalipsky, T.M. Allen, Targeted delivery and triggered release of liposomal doxorubicin enhances cytotoxicity against human B lymphoma cells. Biochim Biophys Acta 1515(2) (2001) 144158.

[192] N.K. Subbarao, R.A. Parente, F.C. Szoka, Jr., L. Nadasdi, K. Pongracz, pHdependent bilayer destabilization by an amphipathic peptide. Biochemistry 26(11) (1987) 2964-2972.

[193] M.J. Turk, J.A. Reddy, J.A. Chmielewski, P.S. Low, Characterization of a novel $\mathrm{pH}$-sensitive peptide that enhances drug release from folate-targeted liposomes at endosomal pHs. Biochim Biophys Acta 1559(1) (2002) 56-68.

[194] P. Simard, J.C. Leroux, pH-sensitive immunoliposomes specific to the CD33 cell surface antigen of leukemic cells. Int J Pharm 381(2) (2009) 86-96.

[195] G.A. Koning, A.M. Eggermont, L.H. Lindner, T.L. Ten Hagen, Hyperthermia and thermosensitive liposomes for improved delivery of chemotherapeutic drugs to solid tumors. Pharm Res 27(8) (2010) 1750-1754.

[196] M.B. Yatvin, J.N. Weinstein, W.H. Dennis, R. Blumenthal, Design of liposomes for enhanced local release of drugs by hyperthermia. Science 202(4374) (1978) 1290-1293.

[197] M.H. Gaber, N.Z. Wu, K. Hong, S.K. Huang, M.W. Dewhirst, D.

Papahadjopoulos, Thermosensitive liposomes: extravasation and release of contents in tumor microvascular networks. Int J Radiat Oncol Biol Phys 36(5) (1996) 1177-1187.

[198] D. Needham, M.W. Dewhirst, The development and testing of a new temperaturesensitive drug delivery system for the treatment of solid tumors. Adv Drug Deliv Rev 53(3) (2001) 285-305.

[199] D. Needham, G. Anyarambhatla, G. Kong, M.W. Dewhirst, A new temperaturesensitive liposome for use with mild hyperthermia: characterization and testing in a human tumor xenograft model. Cancer Res 60(5) (2000) 1197-1201.

[200] G. Kong, G. Anyarambhatla, W.P. Petros, R.D. Braun, O.M. Colvin, D. Needham, M.W. Dewhirst, Efficacy of liposomes and hyperthermia in a human tumor xenograft model: importance of triggered drug release. Cancer Res 60(24) (2000) 6950-6957.

[201] R.J. Ho, B.T. Rouse, L. Huang, Target-sensitive immunoliposomes: preparation and characterization. Biochemistry 25(19) (1986) 5500-5506. 
[202] R.J. Ho, B.T. Rouse, L. Huang, Interactions of target-sensitive immunoliposomes with herpes simplex virus. The foundation of a sensitive immunoliposome assay for the virus. J Biol Chem 262(29) (1987) 13979-13984.

[203] R.J. Ho, B.T. Rouse, L. Huang, Target-sensitive immunoliposomes as an efficient drug carrier for antiviral activity. J Biol Chem 262(29) (1987) 13973-13978.

[204] P. Pinnaduwage, L. Huang, Stable target-sensitive immunoliposomes. Biochemistry 31(11) (1992) 2850-2855.

[205] H. Alkan-Onyuksel, S.M. Demos, G.M. Lanza, M.J. Vonesh, M.E. Klegerman, B.J. Kane, J. Kuszak, D.D. McPherson, Development of inherently echogenic liposomes as an ultrasonic contrast agent. J Pharm Sci 85(5) (1996) 486-490.

[206] S. Huang, A.J. Hamilton, S.D. Tiukinhoy, A. Nagaraj, B.J. Kane, M. Klegerman, D.D. McPherson, R.C. MacDonald, Liposomes as ultrasound imaging contrast agents and as ultrasound-sensitive drug delivery agents. Cell Mol Biol Lett 7(2) (2002) 233-235.

[207] J.A. Kopechek, T.M. Abruzzo, B. Wang, S.M. Chrzanowski, D.A. Smith, P.H. Kee, S. Huang, J.H. Collier, D.D. McPherson, C.K. Holland, Ultrasoundmediated release of hydrophilic and lipophilic agents from echogenic liposomes. J Ultrasound Med 27(11) (2008) 1597-1606.

[208] J.H. Crowe, L.M. Crowe, D. Chapman, Preservation of membranes in anhydrobiotic organisms: the role of trehalose. Science 223(4637) (1984) 701703.

[209] J.H. Crowe, F.A. Hoekstra, K.H. Nguyen, L.M. Crowe, Is vitrification involved in depression of the phase transition temperature in dry phospholipids? Biochim Biophys Acta 1280(2) (1996) 187-196.

[210] S. Ohtake, C. Schebor, J.J. de Pablo, Effects of trehalose on the phase behavior of DPPC-cholesterol unilamellar vesicles. Biochim Biophys Acta 1758(1) (2006) 65-73.

[211] J.L. Green, C.A. Angell, Phase relations and vitrification in saccharide-water solutions and the trehalose anomaly. J Phys Chem 93(8) (1989) 2880-2882.

[212] K.L. Koster, M.S. Webb, G. Bryant, D.V. Lynch, Interactions between soluble sugars and POPC (1-palmitoyl-2-oleoylphosphatidylcholine) during dehydration: vitrification of sugars alters the phase behavior of the phospholipid. Biochim Biophys Acta 1193(1) (1994) 143-150.

[213] K.L. Koster, Glass formation and desiccation tolerance in seeds. Plant Physiol 96(1) (1991) 302-304.

[214] J.S. Clegg, The origin of trehalose and its significance during the formation of encysted dormant embryos of artemia salina. Comp Biochem Physiol 14 (1965) 135-143.

[215] J.H. Crowe, K.A.C. Madin, Anhydrobiosis in nematodes: evaporative water loss and survival. J Exp Zool 193(3) (1975) 323-333.

[216] J.H. Crowe, M.A. Whittam, D. Chapman, L.M. Crowe, Interactions of phospholipid monolayers with carbohydrates. Biochim Biophys Acta 769(1) (1984) 151-159.

[217] L.M. Crowe, J.H. Crowe, A. Rudolph, C. Womersley, L. Appel, Preservation of freeze-dried liposomes by trehalose. Arch Biochem Biophys 242(1) (1985) 240247. 
[218] J.H. Crowe, B.J. Spargo, L.M. Crowe, Preservation of dry liposomes does not require retention of residual water. Proc Natl Acad Sci USA 84(6) (1987) 15371540.

[219] C. Womersley, P.S. Uster, A.S. Rudolph, J.H. Crowe, Inhibition of dehydrationinduced fusion between liposomal membranes by carbohydrates as measured by fluorescence energy transfer. Cryobiology 23(3) (1986) 245-255.

[220] G. Strauss, P. Schurtenberger, H. Hauser, The interaction of saccharides with lipid bilayer vesicles: stabilization during freeze-thawing and freeze-drying. Biochim Biophys Acta 858(1) (1986) 169-180.

[221] H. Hauser, G. Strauss, Stabilization of small, unilamellar phospholipid vesicles by sucrose during freezing and dehydration. Adv Exp Med Biol 238 (1988) 71-80.

[222] C.S. Pereira, P.H. Hunenberger, Interaction of the sugars trehalose, maltose and glucose with a phospholipid bilayer: a comparative molecular dynamics study. $\mathrm{J}$ Phys Chem B 110(31) (2006) 15572-15581.

[223] C.S. Pereira, P.H. Hunenberger, Effect of trehalose on a phospholipid membrane under mechanical stress. Biophys J 95(8) (2008) 3525-3534.

[224] A.V. Popova, D.K. Hincha, Effects of cholesterol on dry bilayers: interactions between phosphatidylcholine unsaturation and glycolipid or free sugar. Biophys $\mathrm{J}$ 93(4) (2007) 1204-1214.

[225] M. Doxastakis, A.K. Sum, J.J. de Pablo, Modulating membrane properties: the effect of trehalose and cholesterol on a phospholipid bilayer. J Phys Chem B 109(50) (2005) 24173-24181.

[226] J.H. Crowe, L.M. Crowe, Factors affecting the stability of dry liposomes. Biochim Biophys Acta 939(2) (1988) 327-334.

[227] K.L. Koster, Y.P. Lei, M. Anderson, S. Martin, G. Bryant, Effects of vitrified and nonvitrified sugars on phosphatidylcholine fluid-to-gel phase transitions. Biophys J 78(4) (2000) 1932-1946.

[228] E.C. van Winden, W. Zhang, D.J. Crommelin, Effect of freezing rate on the stability of liposomes during freeze-drying and rehydration. Pharm Res 14(9) (1997) 1151-1160.

[229] E.C. van Winden, H. Talsma, D.J. Crommelin, Thermal analysis of freeze-dried liposome-carbohydrate mixtures with modulated temperature differential scanning calorimetry. J Pharm Sci 87(2) (1998) 231-237.

[230] Y. Aso, S. Yoshioka, Effect of freezing rate on physical stability of lyophilized cationic liposomes. Chem Pharm Bull (Tokyo) 53(3) (2005) 301-304.

[231] M. Friede, M.H. Van Regenmortel, F. Schuber, Lyophilized liposomes as shelf items for the preparation of immunogenic liposome-peptide conjugates. Anal Biochem 211(1) (1993) 117-122.

[232] C. Chen, D. Han, Y. Zhang, Y. Yuan, X. Tang, The freeze-thawed and freezedried stability of cytarabine-encapsulated multivesicular liposomes. Int J Pharm 387(1-2) (2010) 147-153.

[233] S.B. Barnett, G.R. ter Haar, M.C. Ziskin, W.L. Nyborg, K. Maeda, J. Bang, Current status of research on biophysical effects of ultrasound. Ultrasound Med Biol 20(3) (1994) 205-218. 
[234] S.L. Huang, A.J. Hamilton, A. Nagaraj, S.D. Tiukinhoy, M.E. Klegerman, D.D. McPherson, R.C. Macdonald, Improving ultrasound reflectivity and stability of echogenic liposomal dispersions for use as targeted ultrasound contrast agents. J Pharm Sci 90(12) (2001) 1917-1926.

[235] K. Temming, R.M. Schiffelers, G. Molema, R.J. Kok, RGD-based strategies for selective delivery of therapeutics and imaging agents to the tumour vasculature. Drug Resist Updat 8(6) (2005) 381-402.

[236] H.R. Desu, Targeted delivery of surface modified nanoparticles: modulation of inflammation for acute lung injury. [Dissertation], [Memphis], The University of Tennessee Health Science Center, (2009), 153 p.

[237] N.A. Williams, T. Dean, Vial breakage by frozen mannitol solutions: correlation with thermal characteristics and effect of stereoisomerism, additives, and vial configuration. J Parenter Sci Technol 45(2) (1991) 94-100.

[238] A.I. Kim, M.J. Akers, S.L. Nail, The physical state of mannitol after freezedrying: effects of mannitol concentration, freezing rate, and a noncrystallizing cosolute. J Pharm Sci 87(8) (1998) 931-935.

[239] A. Pyne, R. Surana, R. Suryanarayanan, Crystallization of mannitol below Tg' during freeze-drying in binary and ternary aqueous systems. Pharm Res 19(6) (2002) 901-908.

[240] C. Telang, L. Yu, R. Suryanarayanan, Effective inhibition of mannitol crystallization in frozen solutions by sodium chloride. Pharm Res 20(4) (2003) 660-667.

[241] R.K. Cavatur, N.M. Vemuri, A. Pyne, Z. Chrzan, D. Toledo-Velasquez, R. Suryanarayanan, Crystallization behavior of mannitol in frozen aqueous solutions. Pharm Res 19(6) (2002) 894-900.

[242] F.J. Martin, D. Papahadjopoulos, Irreversible coupling of immunoglobulin fragments to preformed vesicles. An improved method for liposome targeting. J Biol Chem 257(1) (1982) 286-288.

[243] C.B. Hansen, G.Y. Kao, E.H. Moase, S. Zalipsky, T.M. Allen, Attachment of antibodies to sterically stabilized liposomes: evaluation, comparison and optimization of coupling procedures. Biochim Biophys Acta 1239(2) (1995) 133144.

[244] R. Nallamothu, G.C. Wood, C.B. Pattillo, R.C. Scott, M.F. Kiani, B.M. Moore, L.A. Thoma, A tumor vasculature targeted liposome delivery system for combretastatin A4: design, characterization, and in vitro evaluation. AAPS PharmSciTech 7(2) (2006) E32.

[245] T. Kodama, N. Tomita, S. Horie, N. Sax, H. Iwasaki, R. Suzuki, K. Maruyama, S. Mori, F. Manabu, Morphological study of acoustic liposomes using transmission electron microscopy. J Electron Microsc (Tokyo) (2009).

[246] K.E. Hitchcock, D.N. Caudell, J.T. Sutton, M.E. Klegerman, D. Vela, G.J. PyneGeithman, T. Abruzzo, P.E. Cyr, Y.J. Geng, D.D. McPherson, C.K. Holland, Ultrasound-enhanced delivery of targeted echogenic liposomes in a novel ex vivo mouse aorta model. J Control Release 144(3) (2010) 288-295.

[247] D.H. Kim, M.J. Costello, P.B. Duncan, D. Needham, Mechanical properties and microstructure of polycrystalline phospholipid monolayer shells: novel solid microparticles. Langmuir 19(20) (2003) 8455-8466. 
[248] G. Pu, M.L. Longo, M.A. Borden, Effect of microstructure on molecular oxygen permeation through condensed phospholipid monolayers. J Am Chem Soc 127(18) (2005) 6524-6525.

[249] J.A. Zhang, G. Anyarambhatla, L. Ma, S. Ugwu, T. Xuan, T. Sardone, I. Ahmad, Development and characterization of a novel Cremophor EL free liposome-based paclitaxel (LEP-ETU) formulation. Eur J Pharm Biopharm 59(1) (2005) 177-187.

[250] R. Langer, Drug delivery and targeting. Nature 392(6679 Suppl) (1998) 5-10.

[251] R.M. Straubinger, A. Sharma, M. Murray, E. Mayhew, Novel Taxol formulations: Taxol-containing liposomes. J Natl Cancer Inst Monogr(15) (1993) 69-78.

[252] A. Sharma, R.M. Straubinger, Novel Taxol formulations: preparation and characterization of Taxol-containing liposomes. Pharm Res 11(6) (1994) 889-896.

[253] A. Sharma, E. Mayhew, R.M. Straubinger, Antitumor effect of Taxol-containing liposomes in a Taxol-resistant murine tumor model. Cancer Res 53(24) (1993) 5877-5881.

[254] S.V. Balasubramanian, R.M. Straubinger, Taxol-lipid interactions: Taxoldependent effects on the physical properties of model membranes. Biochemistry 33(30) (1994) 8941-8947.

[255] C. Bernsdorff, R. Reszka, R. Winter, Interaction of the anticancer agent Taxol (paclitaxel) with phospholipid bilayers. J Biomed Mater Res 46(2) (1999) 141149.

[256] L.P. Cavalcanti, O. Konovalov, H. Haas, X-ray diffraction from paclitaxel-loaded zwitterionic and cationic model membranes. Chem Phys Lipids 150(1) (2007) 5865.

[257] R.M. Straubinger, S.V. Balasubramanian, Preparation and characterization of taxane-containing liposomes. Methods Enzymol 391 (2005) 97-117.

[258] P. Crosasso, M. Ceruti, P. Brusa, S. Arpicco, F. Dosio, L. Cattel, Preparation, characterization and properties of sterically stabilized paclitaxel-containing liposomes. J Control Release 63(1-2) (2000) 19-30.

[259] J. Wu, Q. Liu, R.J. Lee, A folate receptor-targeted liposomal formulation for paclitaxel. Int J Pharm 316(1-2) (2006) 148-153.

[260] T. Yang, F.D. Cui, M.K. Choi, J.W. Cho, S.J. Chung, C.K. Shim, D.D. Kim, Enhanced solubility and stability of PEGylated liposomal paclitaxel: in vitro and in vivo evaluation. Int J Pharm 338(1-2) (2007) 317-326.

[261] T. Yang, M.K. Choi, F.D. Cui, J.S. Kim, S.J. Chung, C.K. Shim, D.D. Kim, Preparation and evaluation of paclitaxel-loaded PEGylated immunoliposome. J Control Release 120(3) (2007) 169-177.

[262] D.B. Khismatullin, Resonance frequency of microbubbles: effect of viscosity. J Acoust Soc Am 116(3) (2004) 1463-1473.

[263] H. Zheng, O. Mukdadi, R. Shandas, Theoretical predictions of harmonic generation from submicron ultrasound contrast agents for nonlinear biomedical ultrasound imaging. Phys Med Biol 51(3) (2006) 557-573.

[264] K.W. Ferrara, Driving delivery vehicles with ultrasound. Adv Drug Deliv Rev 60(10) (2008) 1097-1102. 
[265] P.A. Dayton, S. Zhao, S.H. Bloch, P. Schumann, K. Penrose, T.O. Matsunaga, R. Zutshi, A. Doinikov, K.W. Ferrara, Application of ultrasound to selectively localize nanodroplets for targeted imaging and therapy. Mol Imaging 5(3) (2006) 160-174.

[266] K.C. Crowder, M.S. Hughes, J.N. Marsh, A.M. Barbieri, R.W. Fuhrhop, G.M. Lanza, S.A. Wickline, Sonic activation of molecularly-targeted nanoparticles accelerates transmembrane lipid delivery to cancer cells through contact-mediated mechanisms: implications for enhanced local drug delivery. Ultrasound Med Biol 31(12) (2005) 1693-1700.

[267] K. Park, J.H. Kim, Y.S. Nam, S. Lee, H.Y. Nam, K. Kim, J.H. Park, I.S. Kim, K. Choi, S.Y. Kim, I.C. Kwon, Effect of polymer molecular weight on the tumor targeting characteristics of self-assembled glycol chitosan nanoparticles. J Control Release 122(3) (2007) 305-314.

[268] H.Y. Hwang, I.S. Kim, I.C. Kwon, Y.H. Kim, Tumor targetability and antitumor effect of docetaxel-loaded hydrophobically modified glycol chitosan nanoparticles. J Control Release 128(1) (2008) 23-31.

[269] J. Klohs, A. Wunder, K. Licha, Near-infrared fluorescent probes for imaging vascular pathophysiology. Basic Res Cardiol 103(2) (2008) 144-151.

[270] J.V. Frangioni, In vivo near-infrared fluorescence imaging. Curr Opin Chem Biol 7(5) (2003) 626-634.

[271] G.L. Amidon, H. Lennernas, V.P. Shah, J.R. Crison, A theoretical basis for a biopharmaceutic drug classification: the correlation of in vitro drug product dissolution and in vivo bioavailability. Pharm Res 12(3) (1995) 413-420.

[272] M.V. Varma, R. Panchagnula, Enhanced oral paclitaxel absorption with vitamin E-TPGS: effect on solubility and permeability in vitro, in situ and in vivo. Eur J Pharm Sci 25(4-5) (2005) 445-453.

[273] S. Koudelka, P. Turanek-Knotigova, J. Masek, Z. Korvasova, M. Skrabalova, J. Plockova, E. Bartheldyova, J. Turanek, Liposomes with high encapsulation capacity for paclitaxel: preparation, characterization and in vivo anticancer effect. J Pharm Sci 99(5) (2010) 2309-2319.

[274] J.H. Crowe, L.M. Crowe, A.E. Oliver, N. Tsvetkova, W. Wolkers, F. Tablin, The trehalose myth revisited: introduction to a symposium on stabilization of cells in the dry state. Cryobiology 43(2) (2001) 89-105.

[275] P. Sundaramurthi, R. Suryanarayanan, Trehalose crystallization during freezedrying: implications on lyoprotection. J Phys Chem Lett 1 (2010) 510-514. 


\section{VITA}

Vinayagam Kannan was born in Vellore, Tamil Nadu, India in 1972. He received his Bachelor of Pharmacy degree from the Tamil Nadu Dr. MGR Medical University, Chennai, Tamil Nadu, India and his Master of Science degree in Pharmaceutics from National Institute of Pharmaceutical Education and Research (NIPER), SAS Nagar, Punjab, India. He worked as a Research Associate in New Drug Discovery Research (NDDR) division of Ranbaxy Research Laboratories, Gurgaon, Haryana, India for about two years. He enrolled for the Ph.D. program in Pharmaceutical Sciences at The University of Tennessee Health Science Center (UTHSC), Memphis in the year 2005 and joined the Parenteral Medications Laboratories (PML) with Dr. George C. Wood as major advisor.

He is the member of American Association of Pharmaceutical Scientists (AAPS) and Parenteral Drug Association (PDA). He was inducted into The Rho Chi Society in the year 2009 for his academic accomplishments. He also received Best Graduate Teaching Assistant Award in Pharmaceutics for the academic year 2007-2008. He has presented the research work in various international meetings. He was the recipient of PDA's graduate research symposium and pre-doctoral fellowship in the year 2008. After graduation, he plans to pursue his career in formulation research and development. 